DOE/ID-10634

April 1998

\title{
Characterization of Carbon-Based Electrochemical Capacitor Technology from Maxwell Energy Products, Inc.
}

RECEIVED

JUL $O$ Y 1998

OSTI

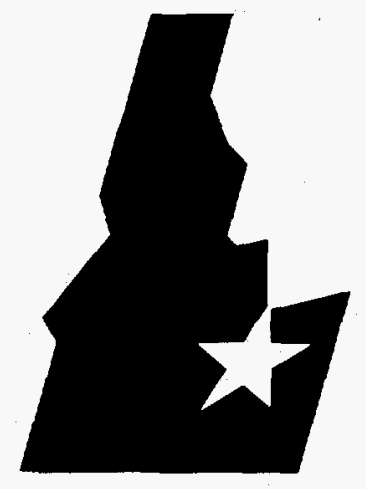

MASTER

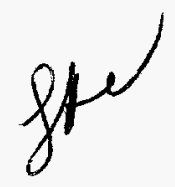

Idaho National Engineering Laboratory

U.S. Department of Energy $\cdot$ /daho Operations Office

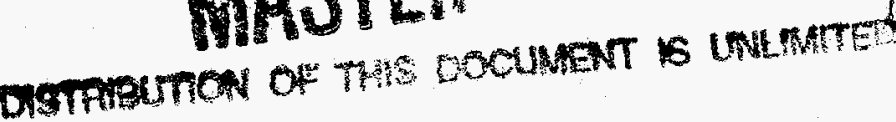
$-4+2$ 
DOE/ID-10634

\section{Characterization of Carbon-Based Electrochemical Capacitor Technology from Maxwell Energy Products, Inc.}

R. B. Wright

T. C. Murphy

April 1998

Prepared for the U.S. Department of Energy

Assistant Secretary for Energy Efficiency and Renewable Energy (EE)

Under DOE Idaho Operations Office

Contract DE-AC07-94ID13223 


\section{DISCLAIMER}

This report was prepared as an account of work sponsored by an agency of the United States Government. Neither the United States Government nor any agency thereof, nor any of their employees, make any warranty, express or implied, or assumes any legal liability or responsibility for the accuracy, completeness, or usefulness of any information, apparatus, product, or process disclosed, or represents that its use would not infringe privately owned rights. Reference herein to any specific commercial product, process, or service by trade name, trademark, manufacturer, or otherwise does not necessarily constitute or imply its endorsement, recommendation, or favoring by the United States Government or any agency thereof. The views and opinions of authors expressed herein do not necessarily state or reflect those of the United States Government or any agency thereof. 


\section{DISCLAIMER}

Portions of this document may be illegible in electronic image products. Images are produced from the best available original document. 


\section{CONTENTS}

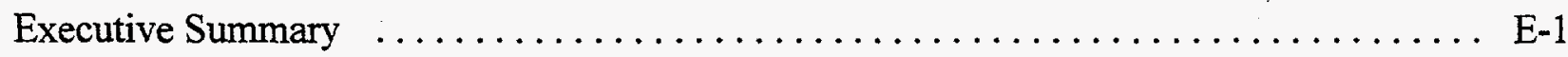

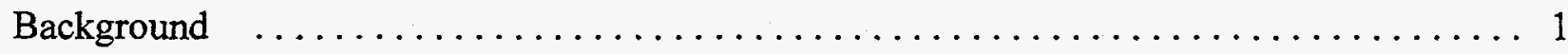

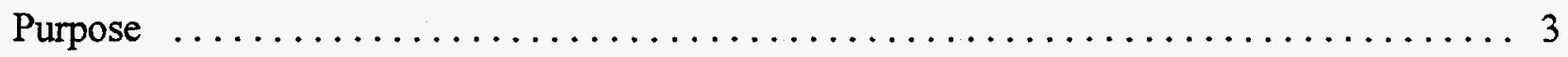

Description of Capacitors and Tests to be Conducted $\ldots \ldots \ldots \ldots \ldots \ldots \ldots \ldots \ldots$

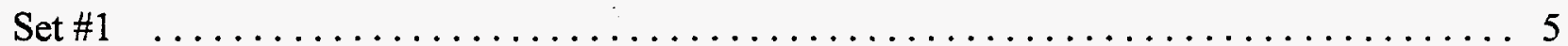

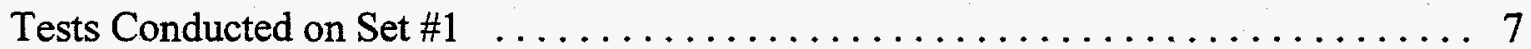

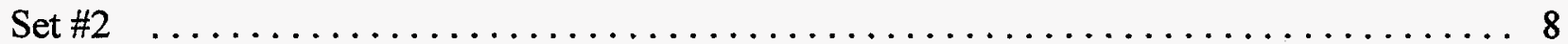

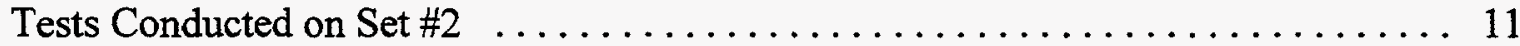

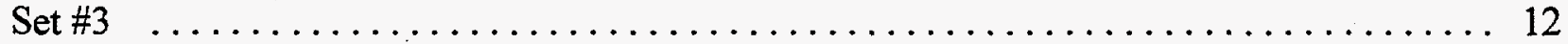

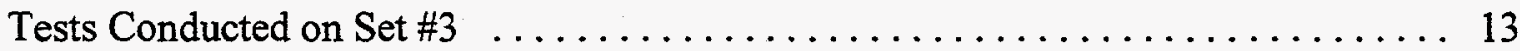

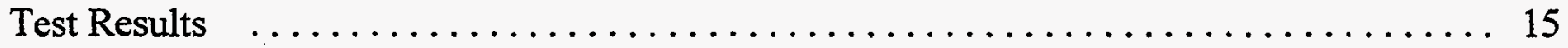

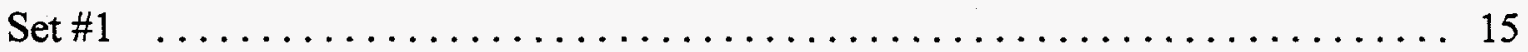

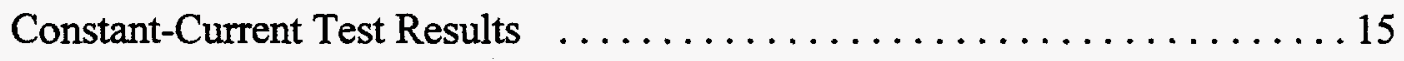

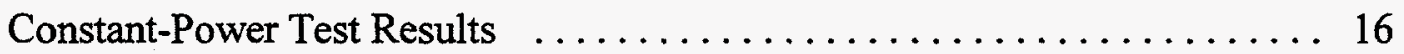

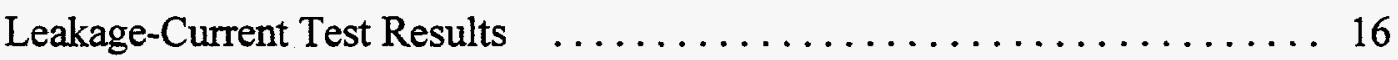

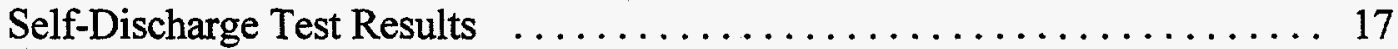

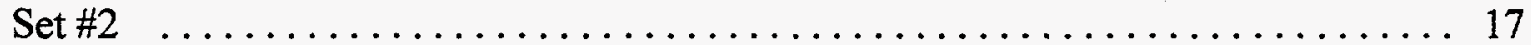

Constant-Current Test Results $\ldots \ldots \ldots \ldots \ldots \ldots \ldots \ldots \ldots \ldots \ldots \ldots \ldots$

ESR and Capacitance Measurements $\ldots \ldots \ldots \ldots \ldots \ldots \ldots \ldots \ldots$

RC-Time Constant Measurements $\ldots \ldots \ldots \ldots \ldots \ldots \ldots \ldots .20$

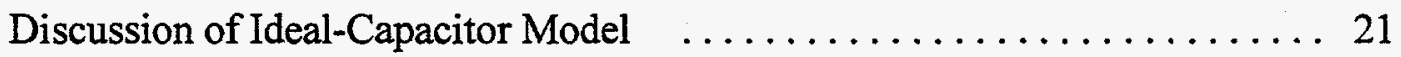

Comparisons to Ideal Capacitor Model $\ldots \ldots \ldots \ldots \ldots \ldots \ldots . \ldots \ldots$

Stored and Extracted Energy from Constant-Current Tests $\ldots \ldots \ldots \ldots .24$

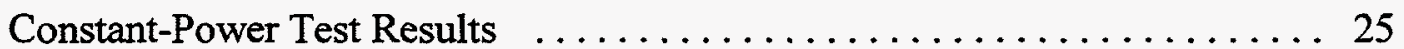

Constant-Current and Constant-Power Discharge Efficiency $\ldots \ldots \ldots \ldots 27$

Leakage-Current Test Results $\ldots \ldots \ldots \ldots \ldots \ldots \ldots \ldots \ldots \ldots . \ldots 29$

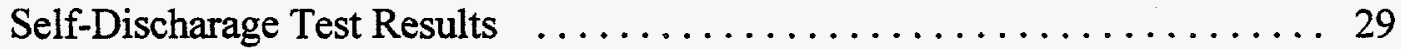

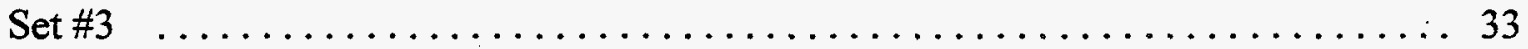

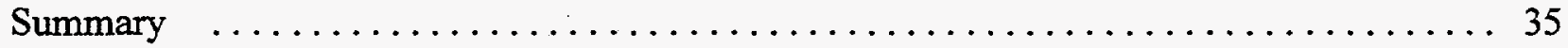

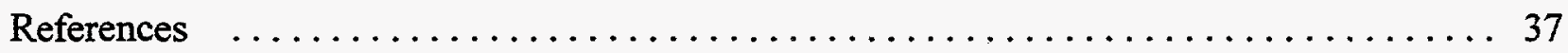




\section{FIGURES}

1. Maxwell Energy Products, Inc. electrochemical capacitor $\# 1143 \ldots \ldots \ldots \ldots \ldots$

2. Maxwell Energy Products, Inc. electrochemical capacitor $\# 1021 \ldots \ldots \ldots \ldots \ldots$.

3. Maxwell Energy Products, Inc. 24 V, 2 F bipolar stack electrochemical

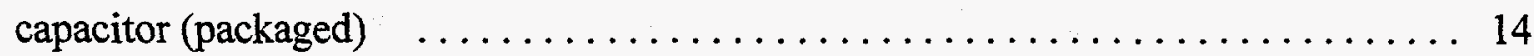

4. Maxwell Energy Products, Inc. 24 V, 2 F bipolar stack electrochemical capacitor (unpackaged)

\section{TABLES}

1. Data supplied by Maxwell Energy Products, Inc. for testing of capacitors of Set \#1 $\quad$. 7

\section{APPENDIX}

A. Graphic Figures for Maxwell Energy Products, Inc. Capacitor Sets \#1, \#2 and \#3 .. A-1

B. Capacitor Testing Fundamentals $\ldots \ldots \ldots \ldots \ldots \ldots \ldots \ldots \ldots \ldots \ldots \ldots \ldots \ldots \ldots$

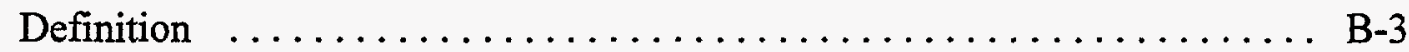

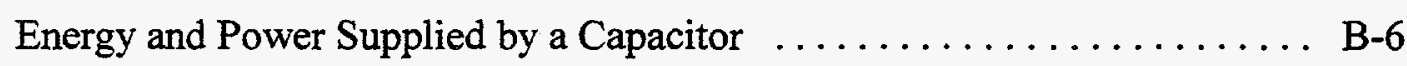

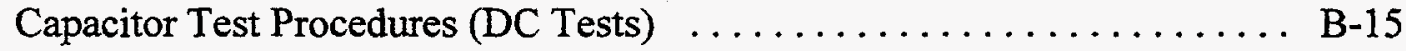

Constant-Current Tests $\ldots \ldots \ldots \ldots \ldots \ldots \ldots \ldots \ldots \ldots \ldots$ B-15

Constant-Power Tests $\ldots \ldots \ldots \ldots \ldots \ldots \ldots \ldots \ldots \ldots \ldots \ldots, B-17$

Leakage-Current Test $\ldots \ldots \ldots \ldots \ldots \ldots \ldots \ldots \ldots \ldots \ldots \ldots$ B-19

Self-Discharge Test (Stand Test) $\ldots \ldots \ldots \ldots \ldots \ldots \ldots \ldots \ldots$ B-19

Life-Cycle Test $\ldots \ldots \ldots \ldots \ldots \ldots \ldots \ldots \ldots \ldots \ldots \ldots \ldots \ldots \ldots \ldots \ldots$ 


\section{EXECUTIVE SUMMARY}

Three sets of capacitors supplied by Maxwell Energy Products, Inc. were received for testing by the INEEL Automotive Systems and Technology Department. All three sets were based on an experimental technology employing a carbon-based electrode system with a nonaqueous electrolyte. Set \#1 consisted of three capacitors (capacitor numbers \#1141,\#1142 and $\# 1143$ ) with nominal ratings of $155 \mathrm{~F}$ to $165 \mathrm{~F}$ at $3 \mathrm{~V}$. Set $\# 2$ consisted of three capacitors (capacitor numbers \#1021, \#1022 and \#1023) that had a nominal rating of $2300 \mathrm{~F}$ at $3 \mathrm{~V}$. Set \#3 consisted of several capacitors with ratings of $2 \mathrm{~F}$ at $24 \mathrm{~V}$.

The test parameters were set according to the ratings of each device and from information supplied by Maxwell Energy Products, Inc.

\section{Set \#1 (Capacitors \#1141, \#1142 and \#1143)}

The measured as-tested weights for the three capacitors were:

$$
\begin{array}{ll}
\# 1141: & 33.20 \mathrm{~g}(0.03320 \mathrm{~kg}) \\
\# 1142: & 33.23 \mathrm{~g}(0.03323 \mathrm{~kg}) \\
\# 1143: & 31.90 \mathrm{~g}(0.03190 \mathrm{~kg})
\end{array}
$$

All of the tests conducted on this set of capacitors were done at a test temperature of $23^{\circ} \mathrm{C}$. The first test that was run on these capacitors was the constant-current test. The constantcurrent test current ranged from $2 \mathrm{~A}$ to $24 \mathrm{~A}$ over the voltage range of $1.5 \mathrm{~V}$ to $3 \mathrm{~V}$ (some of the tests were conducted also over the voltage range of $0 \mathrm{~V}$ to $3 \mathrm{~V}$.) The constant-current test data was discussed in terms of the ideal-capacitor model that assumes a constant capacitance capacitor connected in series with a resistor which is used to model the equivalent series resistance, ESR, of the capacitor. The time dependence of the charge and discharge voltage deviated from the ideal-model and were attributed to a capacitance that depends on the capacitor potential as well as being dependent on the constant-current charge and discharge current. The discharge ESR values for the three capacitors ranged between approximately 9 to 22 milliohms and generally increased with increasing discharge current. The measured capacitance values varied from $150 \mathrm{~F}$ to $163 \mathrm{~F}$ at a $2 \mathrm{~A}$ constant-current discharge, decreasing to $95 \mathrm{~F}$ to $135 \mathrm{~F}$ at a $16 \mathrm{~A}$ discharge.

A series of constant-power tests were also conducted on the three capacitors. Constantpower tests were conducted over the constant-power discharge range of $4.5 \mathrm{~W}$ to $36 \mathrm{~W}$. The specific energy for all three devices was approximately $4.3 \mathrm{~W} \cdot \mathrm{h} / \mathrm{kg}$ at $141 \mathrm{~W} / \mathrm{kg}$, decreasing to approximately $1.9 \mathrm{~W} \cdot \mathrm{h} / \mathrm{kg}$ at $1100 \mathrm{~W} / \mathrm{kg}$. 
The leakage-current test was conducted on capacitors \#1142 and \#1143. It took approximately 20 hours for the leakage-current to stabilize at approximately 500 microamperes for both capacitors. Capacitor \#1143 had a slightly better (i.e., lower) leakage-current. The time dependence of the equivalent parallel resistance (EPR) of the capacitors was determined from the leakage-current test data. The stable EPR of both devices after approximately 20 hours was between 700 and 1000 ohms.

The self-discharge test was also done on the three capacitors. Capacitor \#1142 was the best of the three devices as it had the smallest decrease in voltage over the 72-hour test period. Capacitor \#1143 was next best, followed by \#1141. From these data, the self-discharge energy loss factor (SDLF) was calculated for the three capacitors as a function of test time. Capacitor \#1142 was the best device losing approximately $43 \%$ (after 72 hours) of its initial stored energy due to non-ideal energy storage/transfer processes. Device \#1143 was next best, losing 57\% of its stored energy after 72 hours. Capacitor \#1141 lost $\sim 72 \%$ of its initial stored energy after 72 hours.

\section{Set \#2 (Capacitors \#1021, \#1022 and \#1023)}

The measured, as-tested weights of the three capacitors were:

$$
\begin{array}{ll}
\# 1021: & 622 \mathrm{~g}(0.622 \mathrm{~kg}) \\
\# 1022: & 633 \mathrm{~g}(0.633 \mathrm{~kg}) \\
\# 1023: & 635 \mathrm{~g}(0.635 \mathrm{~kg})
\end{array}
$$

The tests on this set of capacitors were conducted at temperatures of $-20^{\circ} \mathrm{C}, 23^{\circ} \mathrm{C}$ and $55^{\circ} \mathrm{C}$. Constant-current tests were conducted over the charge/discharge range of $10 \mathrm{~A}$ to $300 \mathrm{~A}$. The time dependence of the constant-current charge and discharge voltage was found not to be a linear function of time as predicted by the ideal-capacitor model. These deviations were attributed to a capacitance that varies with the voltage (charge) on the capacitor. Initially, the constant-current tests were run using a $1.5 \mathrm{~V}$ to $3.0 \mathrm{~V}$ voltage range but were then redone using a $\sim 0 \mathrm{~V}$ to $3.0 \mathrm{~V}$ voltage range. From the constant-current test data the equivalent series resistance (ESR) and capacitance values as a function of the constant current test current were calculated. At $23^{\circ} \mathrm{C}$, the ESR values ranged from 1 to 1.2 milliohms at $10 \mathrm{~A}$, decreasing to 0.3 to 0.4 milliohms at $100 \mathrm{~A}$. The $55^{\circ} \mathrm{C} \mathrm{ESR}$ values varied from 0.45 to 0.65 milliohms at $10 \mathrm{~A}$; at $200 \mathrm{~A}$ the values ranged from 0.55 to 0.8 milliohms. The ESR values increased considerably at $-20^{\circ} \mathrm{C}$ (only capacitor \#1022 was tested at this temperature) where at $10 \mathrm{~A}$ the ESR was 2.35 milliohms, increasing to 3.6 milliohms at $100 \mathrm{~A}$ which then decreased to 1.6 milliohms at $250 \mathrm{~A}$. 
It was observed that a large difference existed between the capacitance calculated from the $3 \mathrm{~V}$ to $1.5 \mathrm{~V}$ discharge range data as compared with that calculated from the $3 \mathrm{~V}$ to $\sim 0 \mathrm{~V}$ discharge range data. This discrepancy was because the discharge voltage was not a linear function of the discharge time due to the capacitance of the device not being a constant, but dependent on the voltage of the capacitor. In general, the capacitance of the three capacitors decreased with increasing discharge current in a somewhat linear fashion. Using the $3 \mathrm{~V}$ to 0.01 $\mathrm{V}$ values at a $23^{\circ} \mathrm{C}$ test temperature, the average capacitance of the three capacitors was approximately $2400 \mathrm{~F}$ at a $10 \mathrm{~A}$ discharge, decreasing to approximately $2275 \mathrm{~F}$ at a discharge current of $300 \mathrm{~A}$. The capacitance as a function of the constant-current discharge current at the $-20^{\circ} \mathrm{C}$ and $55^{\circ} \mathrm{C}$ test temperatures were also determined. A large difference was noted upon comparing the capacitance values for tests where the discharge voltage was from $3 \mathrm{~V}$ to $1.5 \mathrm{~V}$ and for those tests where the discharge voltage was $3 \mathrm{~V}$ to $0.05 \mathrm{~V}$. For example, for capacitor \#1022 at a 10 A constant-current discharge for a $3 \mathrm{~V}$ to $1.5 \mathrm{~V}$ discharge range, the capacitance was $2473.2 \mathrm{~F}$ as compared to $2300.9 \mathrm{~F}$ measured for the $3 \mathrm{~V}$ to $0.05 \mathrm{~V}$ test range. At a $200 \mathrm{~A}$ constant-current discharge the capacitance for the $3 \mathrm{~V}$ to $1.5 \mathrm{~V}$ discharge range was $200.7 \mathrm{~F}$, but for the $3 \mathrm{~V}$ to $0.05 \mathrm{~V}$ range it was determined to be $1216.7 \mathrm{~F}$. It has become quite clear from the capacitor testing which has been done at the INEEL that the concept of a constant value for the capacitance of a capacitor that does not depend on the voltage (and charge) on the capacitor is not correct and should be more closely examined when reporting the capacitance values of a capacitor. The capacitance values were generally the highest at $23^{\circ} \mathrm{C}$ decreasing slightly when the temperature was increased to $55^{\circ} \mathrm{C}$. When the temperature was decreased to $-20^{\circ} \mathrm{C}$, the capacitance values displayed a more significant decrease. At all of the test temperatures the capacitance decreased in a fairly linear manner as the constant-current discharge current increased. The extent of the decrease with increasing discharge current was the greatest at $20^{\circ} \mathrm{C}$, followed by those at $23^{\circ} \mathrm{C}$. The extent of decrease of the capacitance with increasing discharge current was the smallest at the $55^{\circ} \mathrm{C}$ test temperature.

Knowing the values of the ESR and capacitance as a function of the constant-current discharge current allows the calculation of the capacitor's RC-time constant and its dependence on the discharge current for the capacitor. The RC-time constant is a measure of how fast the voltage and charge of the capacitor decrease when the capacitor is connected to an external load, or if the capacitor is short-circuited. In brief, a smaller RC-time constant allows the capacitor to charge and discharge faster. The RC-time constant also enters considerations concerning how rapidly energy can be extracted from the capacitor and also how fast power can be provided by the capacitor. The RC time constants were determined not to be linear functions of the constantcurrent discharge current and decreased as the discharge current increased because both the ESR and capacitance generally decreased with increasing discharge current. At $23^{\circ} \mathrm{C}$, the RC values are essentially the same for all three capacitors over the $10 \mathrm{~A}$ to $100 \mathrm{~A}$ discharge current range 
with values decreasing from 2.6-3.1 seconds to $0.8-1.0$ seconds. Usually, the RC-time constant decreased with increasing operating temperature. This general trend was also observed in the data at temperatures of $-20^{\circ} \mathrm{C}$ and $23^{\circ} \mathrm{C}$ (capacitor \#1022) for those tests that used a discharge voltage range of $3 \mathrm{~V}$ to $0.01 \mathrm{~V}$. For a $10 \mathrm{~A}$ constant-current discharge the $\mathrm{RC}$ values were 3.3 seconds at $23^{\circ} \mathrm{C}$ which increased to 4.7 seconds at $-20^{\circ} \mathrm{C}$. At a $100 \mathrm{~A}$ discharge, $\mathrm{RC}$ was equal to 1 second at $23^{\circ} \mathrm{C}$ and increased to 6.4 seconds at $-20^{\circ} \mathrm{C}$. All three capacitors were tested at $55^{\circ} \mathrm{C}$ using a voltage discharge range of $3 \mathrm{~V}$ to $1.5 \mathrm{~V}$ and from these tests the corresponding RC time constants were determined as a function of discharge current. The $\mathrm{RC}$ values at $55^{\circ} \mathrm{C}$ are smaller at the lower discharge currents $(<50 \mathrm{~A})$ than at $23^{\circ} \mathrm{C}$, but are somewhat larger at the 100 A discharge level.

The issue of how well the ideal-capacitor model fit the charge/discharge data and also how valid is the assumption of a constant capacitance for the capacitors was examined in further detail. The experimental data showed the voltage dependence of the capacitance quite dramatically as it illustrated that under extreme conditions of test temperature and high charge/discharge rates that the capacitor's capacitance can vary widely from that determined assuming that the capacitance is constant, independent of the capacitor's voltage (or charge). The voltage dependence of the capacitance as a function of the capacitor's voltage at the different constant-current discharge currents and the three different test temperatures of $-20^{\circ} \mathrm{C}, 23^{\circ} \mathrm{C}$ and $55^{\circ} \mathrm{C}$ was calculated and discussed.

Using the discharge ESR, $R_{d}$, and the discharge capacitance, $C_{d}$, as functions of the discharge current, $I_{d}$, as measured from the experimental constant-current discharge data, the total energy that can be extracted from the capacitor, not including that removed by the voltage drop due to $I_{d} R_{d}$, was calculated. The maximum extractable energy $=(1 / 2) C_{d}\left(3-I_{d} R_{d}\right)^{2}$, where the 3 in the expression arises due the fact that the capacitors were charged to $3 \mathrm{~V}$, as a function of the constant-current discharge current for capacitor $\# 1022$ for test temperatures of $-20^{\circ} \mathrm{C}, 23^{\circ} \mathrm{C}$ and $55^{\circ} \mathrm{C}$ was determined. This energy was shown to be the highest at $55^{\circ} \mathrm{C}$, which decreased at $23^{\circ} \mathrm{C}$ and further decreased at $-20^{\circ} \mathrm{C}$. The $55^{\circ} \mathrm{C}$ energy decreased fairly linearly with increasing discharge current; that at $23^{\circ} \mathrm{C}$ was somewhat nonlinear, and at $-20^{\circ} \mathrm{C}$ the decrease is quite nonlinear. From the data collected during the constant-current tests it was also possible to determine the energy extracted during the constant-current discharge tests as a function of the discharge current. This measured energy includes that lost due to the $I_{d}{ }^{2} R_{d}$ power losses arising from the ESR of the capacitor, as well as the energy extracted during the constant-current discharge test. The measured energy extracted from capacitor $\# 1022$ at $-20^{\circ} \mathrm{C}, 23^{\circ} \mathrm{C}$ and $55^{\circ} \mathrm{C}$ as a function of discharge current was evaluated over two discharge voltage ranges for $-20^{\circ} \mathrm{C}(3$ $\mathrm{V}$ to $0.05 \mathrm{~V}$, and $3 \mathrm{~V}$ to $1.5 \mathrm{~V})$, over two voltage ranges at $23^{\circ} \mathrm{C}(3 \mathrm{~V}$ to $0.01 \mathrm{~V}$, and $3 \mathrm{~V}$ to 1.5 $\mathrm{V}$ ), and over just one voltage range at $55^{\circ} \mathrm{C}$ (the only constant-current tests conducted at this 
temperature were over a voltage range of $3 \mathrm{~V}$ to $1.5 \mathrm{~V}$ ). All of the measured energy extracted values decreased with increasing discharge current. They decreased fairly linearly with increasing discharge current for the $23^{\circ} \mathrm{C}$ and $55^{\circ} \mathrm{C}$ temperatures, but decreased nonlinearly at $20^{\circ} \mathrm{C}$. Those energy values measured at $-20^{\circ} \mathrm{C}$ and $23^{\circ} \mathrm{C}$ over the two voltage test ranges follow closely the expected $3 / 4$ decrease in extracted energy when the voltage range is $3 \mathrm{~V}$ to $1.5 \mathrm{~V}$ as compared with when it is $3 \mathrm{~V}$ to $\sim 0 \mathrm{~V}$. These results indicate that in order for these capacitors to supply the maximum amount of energy is to operate them at the higher temperatures. However, caution presumably would need to be exercised not to exceed the decomposition temperature of the electrolyte.

Constant-power tests were conducted on the capacitors from $10 \mathrm{~W}$ to $330 \mathrm{~W}$ at temperatures of $-20^{\circ} \mathrm{C}, 23^{\circ} \mathrm{C}$ and $55^{\circ} \mathrm{C}$. At $23^{\circ} \mathrm{C}$ the specific energy of the capacitors varied between approximately $3.85 \mathrm{~W} \cdot \mathrm{h} / \mathrm{kg}$ to $3.98 \mathrm{~W} \cdot \mathrm{h} / \mathrm{kg}$ at approximately $15.7 \mathrm{~W} / \mathrm{kg}$, decreasing in a fairly linear manner to approximately $3.5 \mathrm{~W} \cdot \mathrm{h} / \mathrm{kg}$ to $3.6 \mathrm{~W} \cdot \mathrm{h} / \mathrm{kg}$ at approximately $530 \mathrm{~W} / \mathrm{kg}$. The specific energy volumetric density as a function of the specific power volumetric density were also determined for the three capacitors at $23^{\circ} \mathrm{C}$. These values were calculated using the volume of the capacitor calculated from the dimensions supplied by Maxwell Energy Products, Inc. (i.e., 0.462 liters). The specific energy volumetric density varied from $5.24 \mathrm{~W} \cdot \mathrm{h} / \mathrm{L}$ to $5.5 \mathrm{~W} \cdot \mathrm{h} / \mathrm{l}$ at 21.7 $\mathrm{W} / \mathrm{L}$, decreasing to $4.73 \mathrm{~W} \cdot \mathrm{h} / \mathrm{L}$ to $4.95 \mathrm{~W} \cdot \mathrm{h} / \mathrm{L}$ at $714.8 \mathrm{~W} / \mathrm{L}$ at $23^{\circ} \mathrm{C}$. Constant-power tests were also conducted at temperatures of $55^{\circ} \mathrm{C}$ on the capacitors in addition to the $23^{\circ} \mathrm{C}$ tests. Constantpower tests at $10 \mathrm{~W}, 20 \mathrm{~W}, 50 \mathrm{~W}, 100 \mathrm{~W}, 200 \mathrm{~W}, 250 \mathrm{~W}$ and $330 \mathrm{~W}$ (a constant-current of $10 \mathrm{~A}$ was used to charge the capacitor) at a temperature of $55^{\circ} \mathrm{C}$ over the discharge voltage range of 3 $\mathrm{V}$ to $1.5 \mathrm{~V}$ were carried out. The same sequence of constant-power tests $(10 \mathrm{~W}, 20 \mathrm{~W}, 50 \mathrm{~W}, 100 \mathrm{~W}, 200 \mathrm{~W}, 250 \mathrm{~W}$ and $330 \mathrm{~W})$ at a test temperature of $-20^{\circ} \mathrm{C}$ over the discharge voltage range of $3 \mathrm{~V}$ to $1.5 \mathrm{~V}$ were also conducted on capacitor \#1022. As before, a 10 A constant-current charge was used on these tests. The highest specific energy was observed to occur at $23^{\circ} \mathrm{C}$. The specific energy was lower at $55^{\circ} \mathrm{C}$ and it also decreased more rapidly with increasing specific power than was observed at $23^{\circ} \mathrm{C}$. The specific energy and power values at $55^{\circ} \mathrm{C}$ for the three capacitors ranged from $3.6 \mathrm{~W} \cdot \mathrm{h} / \mathrm{kg}$ to $3.8 \mathrm{~W} \cdot \mathrm{h} / \mathrm{kg}$ at $15.7 \mathrm{~W} / \mathrm{kg}$, decreasing in a fairly linear manner to $3.0 \mathrm{~W} \cdot \mathrm{h} / \mathrm{kg}$ to $3.4 \mathrm{~W} \cdot \mathrm{h} / \mathrm{kg}$ at $520 \mathrm{~W} / \mathrm{kg}$. At $-20^{\circ} \mathrm{C}$, the specific energy (capacitor \#1022) was reduced even further. The specific energy at $-20^{\circ} \mathrm{C}$ decreased quite rapidly with increasing specific power compared with those at the higher test temperatures. At this temperature, the value of the specific energy was $3.6 \mathrm{~W} \cdot \mathrm{h} / \mathrm{kg}$ at a specific power of $15.8 \mathrm{~W} / \mathrm{kg}$, decreasing linearly to $0.98 \mathrm{~W} \cdot \mathrm{h} / \mathrm{kg}$ at $521 \mathrm{~W} / \mathrm{kg}$.

Constant-current and constant-power test data were used to calculate the discharge/charge efficiencies for the capacitors at the three different test temperatures. The highest measured constant-current efficiency of $\sim 1$ was observed at a charge/discharge current of $100 \mathrm{~A}$ at $55^{\circ} \mathrm{C}$. 
The $55^{\circ} \mathrm{C}$ efficiencies were observed to increase with increasing charge/discharge current, reaching a maximum at $100 \mathrm{~A}$, and then decreased with further increases in the charge/discharge current. Over the charge/discharge current test range of $10 \mathrm{~A}$ to $100 \mathrm{~A}$, the efficiencies at $23^{\circ} \mathrm{C}$ decreased with increasing charge/discharge current, decreasing from $\sim 94 \%$ at $10 \mathrm{~A}$ to $\sim 90 \%$ at $100 \mathrm{~A}$. The $-20^{\circ} \mathrm{C}$ efficiencies were lower than those at the higher temperatures and decreased very rapidly with increasing charge/discharge current. At 10 A the efficiency was $\sim 92 \%$, decreasing to $\sim 32 \%$ at $250 \mathrm{~A}$. The round-trip constant-power discharge efficiencies were determined from the constant-power tests. In this instance, a constant-current charge of $10 \mathrm{~A}$ was used in the tests and a series of constant-power discharges were then studied. Analyzing the constant-power tests for capacitor \#1022 at the three test temperatures, the efficiencies were determined as a function of the constant-power discharge power. In this case, the highest efficiencies were measured for those tests conducted at $23^{\circ} \mathrm{C}$ where the efficiency was $\sim 98 \%$ at $10 \mathrm{~W}$, increasing to a maximum of $-99 \%$ at $50 \mathrm{~W}$ and then decreasing to $\sim 96 \%$ at $100 \mathrm{~W}$. At $55^{\circ} \mathrm{C}$, the efficiency was a maximum at $20 \mathrm{~W}(\sim 96 \%)$ which decreased to an efficiency of $\sim 85 \%$ at $330 \mathrm{~W}$. The efficiency at $-20^{\circ} \mathrm{C}$ was a maximum at $20 \mathrm{~W}(\sim 96 \%)$ which decreased quite rapidly to $\sim 25 \%$ at $330 \mathrm{~W}$. Further considerations of the efficiencies associated with the constant-current discharge of a capacitor are developed in an appendix to this report where relationships between the energy that can be provided by a capacitor at a given efficiency and the energy stored in it are derived and discussed. The power that can be provided by the capacitor at a given efficiency and the energy stored in the capacitor as a function of the efficiency are also given in the appendix. Assuming that for practical applications an efficiency of $90 \%$ is desired, by using equations developed in the appendix and the experimental values for the constantcurrent discharge current dependence of the discharge ESR, the corresponding maximum power that can be produced by capacitor $\# 1022$ at temperatures of $-20^{\circ} \mathrm{C}, 23^{\circ} \mathrm{C}$ and $55^{\circ} \mathrm{C}$ were determined. The highest power available at an efficiency of $90 \%$ from capacitor $\# 1022$ at constant-current discharges $<100 \mathrm{~A}$ would be provided if the capacitor was operated at $55^{\circ} \mathrm{C}$. At a discharge current of $100 \mathrm{~A}$, the highest power at $90 \%$ efficiency would occur if the capacitor was at $23^{\circ} \mathrm{C}$. The lowest discharge power at $90 \%$ efficiency would be if the capacitor was at $20^{\circ} \mathrm{C}$, but the trend of the data was for an increasing power at currents greater than $250 \mathrm{~A}$. The corresponding energy that could be provided by capacitor \#1022 at the three test temperatures at $90 \%$ efficiency as a function of the constant-current discharge was also calculated. The maximum energy that could be provided from capacitor \#1022 would be if it were operated at $55^{\circ} \mathrm{C}$. At a 10 A constant-current discharge, the energy would be $\sim 2.82 \mathrm{~W} \cdot \mathrm{h}$, decreasing to $\sim 2.53 \mathrm{~W} \cdot \mathrm{h}$ at $200 \mathrm{~A}$. A lower amount of energy would be available at $23^{\circ} \mathrm{C}$ where at $10 \mathrm{~A}$ the energy is $2.67 \mathrm{~W} \cdot \mathrm{h}$, decreasing to $\sim 2.56 \mathrm{~W} \cdot \mathrm{h}$ at $100 \mathrm{~A}$. At $-20^{\circ} \mathrm{C}$, the available energy decreases 
even further where at $10 \mathrm{~A}$ the energy at $90 \%$ efficiency is $2.58 \mathrm{~W} \cdot \mathrm{h}$, that would decrease to $\sim 1.34 \mathrm{~W} \cdot \mathrm{h}$ at a discharge current of $250 \mathrm{~A}$. At all three test temperatures the amount of available energy at $90 \%$ efficiency decreased in a somewhat linear manner as the constant-current discharge current increased.

Leakage-current tests, which measure the current required to maintain, in this case, a voltage of $3 \mathrm{~V}$ on the capacitor, were conducted on the three capacitors only at $23^{\circ} \mathrm{C}$. Capacitor \#1021 was not very good as its leakage current stabilized at 85 milliamperes after $\sim 8$ hours, while devices \#1022 and \#1023 had leakage currents of approximately 10 milliamperes after $\sim 21$ hours. The corresponding equivalent parallel resistance (EPR) values were calculated from these data. Devices \#1022 and \#1023 were essentially the same, having an EPR value of $\sim 300$ ohms after 24 hours on test while that of \#1021 was 3.5 ohms after 8 hours (the total test time on this particular capacitor). Compared with the previously discussed values of the ESR that were in the milliohm range, it is a good approximation to neglect the EPR when discussing the ideal-capacitor model as applied to constant-current and constant-power tests for these three capacitors.

The self-discharge test, which measures the voltage decrease of the capacitor, and therefore the remaining energy, as a function of time when it is not connected to a load, was conducted on all three capacitors at $23^{\circ} \mathrm{C}$ and $55^{\circ} \mathrm{C}$, and on capacitor $\# 1022$ at $-20^{\circ} \mathrm{C}$. At $23^{\circ} \mathrm{C}$, capacitor \#1021 exhibited considerably worse performance than devices \#1022 and \#1023 as its voltage decreased to $\sim 1.4 \mathrm{~V}$ after 48 hours while the voltage of capacitor \#1022 decreased to $\sim 2.5 \mathrm{~V}$; the voltage of capacitor $\# 1023$ decreased to $\sim 2.65 \mathrm{~V}$. The self-discharge test not only provides information concerning the rate at which the voltage on a capacitor declines with time in the open-circuit state and, thereby, the amount of energy left stored in the capacitor, but the test data can also be analyzed to determine what physical and/or chemical mechanisms may be responsible for the observed voltage decrease. Practical capacitors, like batteries, can suffer selfdischarge over a period of time so that this phenomenon is of major interest in the evaluation of capacitor (and battery) performance and does influence the materials and fabrication methods used in their production. Three potential causes of self-discharge that have been identified in the literature were discussed and used to analyze the collected self-discharge data. The test data analyzed using the three models were found not too correlate with the models over the entire self-discharge voltage range, but perhaps there may be limited voltage and time ranges where some of the models may be applicable. A more extensive analysis of the data that uses various voltage/time ranges fit to the three models may yield more definitive correlations, but this was not done in the present study. 
The self-discharge data was also used to determine the Self-Discharge Energy Loss Factor (SDLF), which is a measure of how much energy has been lost from the capacitor as a function of self-discharge time due to the self-discharge process. At a temperature of $23^{\circ} \mathrm{C}$, the best capacitor was \#1023 that lost $\sim 22 \%$ after 48 hours of the initial stored energy it had at $3 \mathrm{~V}$. The next best device was \#1022 that lost $\sim 32 \%$ of its initial stored energy after 48 hours, followed by capacitor \#1023 that lost $\sim 78 \%$ of its stored energy after this same period. The selfdischarge test was also done on all three of the capacitors at $55^{\circ} \mathrm{C}$. At this test temperature, capacitor \#1023 again displayed the lowest amount of energy loss, $33 \%$ after 48 hours, higher than the $22 \%$ loss is had at $23^{\circ} \mathrm{C}$. Capacitor $\# 1022$ lost $\sim 35 \%$ at $55^{\circ} \mathrm{C}$ compared with the $\sim 32 \%$ it lost at $23^{\circ} \mathrm{C}$. Capacitor \#1021 was again the worst of the three capacitors at this temperature losing $\sim 72 \%$ of its stored energy after 48 hours. Its performance, however, was better at $55^{\circ} \mathrm{C}$ than at $23^{\circ} \mathrm{C}$ where it lost $\sim 78 \%$ of its stored energy after the 48 -hour test period. The selfdischarge test was also done on capacitor $\# 1022$ at $-20^{\circ} \mathrm{C}$ where, after 48 hours, it lost $\sim 16 \%$ of its stored energy compared to having lost $\sim 32 \%$ at $23^{\circ} \mathrm{C}$ and $-35 \%$ at $55^{\circ} \mathrm{C}$. This is quite an improvement compared with the two higher test temperatures. The self-discharge energy loss factor can be used to determine how much energy the capacitor loses as a function of time after it has been charged to its working voltage and then left in an open-circuit state. The total potential energy stored in a capacitor is equal to $E(t)=1 / 2 \mathrm{CV}(\mathrm{t})^{2}$ where $E$ is the energy in joules stored in the capacitor as a function of time, $C$ is the capacitance of the capacitor in farads, and $V(t)$ is the voltage on the capacitor at a time $t$ after it has been charged to its initial voltage and left in an open-circuit condition. A decrease in the voltage on the capacitor will, then, have a dramatic effect on its total stored energy at a latter time, $t$. If one assumes that the capacitance does not depend on the voltage on the capacitor or on the discharge current, assumptions shown not really to be the case for capacitor \#1022, then the energy remaining in the capacitor can be calculated using the time dependent self-discharge voltage in the relation $E_{\text {remaining }}=1 / 2 \mathrm{CV}(\mathrm{t})^{2}$. Using the capacitance values measured for capacitor \#1022 from the 10 A constant-current discharge tests at the three temperatures, i.e., $\mathrm{C}=2302.7 \mathrm{~F}$ at $-20^{\circ} \mathrm{C}, \mathrm{C}=2373.3 \mathrm{~F}$ at $23^{\circ} \mathrm{C}$, and $\mathrm{C}=2503.9 \mathrm{~F}$ at $55^{\circ} \mathrm{C}$, the energy remaining in the capacitor as a function of time during self-discharge was calculated. Initially, at time $\mathrm{t}=0$, the capacitor had the greatest amount of stored energy at $55^{\circ} \mathrm{C}$ $(3.13 \mathrm{~W} \cdot \mathrm{h})$ due to its having the highest capacitance at this temperature. The amount of stored energy at time $\mathrm{t}=0$ decreased as the temperature was lowered, $2.97 \mathrm{~W} \cdot \mathrm{h}$ at $23^{\circ} \mathrm{C}$, and $2.87 \mathrm{~W} \cdot \mathrm{h}$ at $-20^{\circ} \mathrm{C}$. However, as the self-discharge test time increased, at $\sim 6$ hours the energy remaining in the capacitor at $-20^{\circ} \mathrm{C}$ was approximately the same as that stored when the capacitor was at $55^{\circ} \mathrm{C}$. For times greater than $\sim 6$ hours, the energy stored in the capacitor was always greater at $-20^{\circ} \mathrm{C}$ than it was at either $23^{\circ} \mathrm{C}$ or $55^{\circ} \mathrm{C}$. After 48 hours, the energy remaining in the capacitor was $\sim 2.43 \mathrm{~W} \cdot \mathrm{h}$ at $-20^{\circ} \mathrm{C},-2.00 \mathrm{~W} \cdot \mathrm{h}$ at $23^{\circ} \mathrm{C}$, and $-2.06 \mathrm{~W} \cdot \mathrm{h}$ at $55^{\circ} \mathrm{C}$. These values correspond to a decrease in the stored energy of $\sim 15 \%$ at $-20^{\circ} \mathrm{C}, \sim 33 \%$ at $23^{\circ} \mathrm{C}$, and $-34 \%$ at $55^{\circ} \mathrm{C}$, not inappreciable amounts after only 48 hours under open-circuit conditions. Consideration of the 
self-discharge energy loss could be critical for energy storage applications of these capacitors where some provision to periodically recharge the capacitor is not allowed. The self-discharge test data at a test temperature of $23^{\circ} \mathrm{C}$ for capacitor \#1142 from capacitor Set \#1 and capacitor \#1023 from capacitor Set \#2 were also compared. Each of these capacitors exhibited the best performance in the during the self-discharge test, i.e., they each had the smallest amount of voltage decrease in the two sets of capacitors. Capacitor \#1142 exhibited a more rapid falloff of its voltage than capacitor \#1023. Capacitor \#1142 had a considerable lower capacitance, $\sim 157 \mathrm{~F}$, compared with $\sim 2464 \mathrm{~F}$ for capacitor \#1023, which does not necessarily mean that its voltage should decline more rapidly. Comparing the amount of energy lost to self-discharge, capacitor \#1142 lost $~ 38 \%$ of its initial stored energy at $3 \mathrm{~V}$ after 48 hours while capacitor \#1023 lost $\sim 22 \%$ of its initial stored energy at $3 \mathrm{~V}$ also after 48 hours. Based on the amount of energy lost as a function of time under open-circuit conditions, capacitor \#1023 is better than \#1142. As we do not know the details of the differences in the electrode materials, electrolytes and fabrication techniques used in these two sets of capacitors, we cannot at this time attribute this better performance to any particular cause or physical/chemical process(es).

\section{$\underline{\text { Set \#3 }}$}

This set of consisted of eight-cell, $24 \mathrm{~V}$ packaged and unpackaged devices. They were rated at $2 \mathrm{~F}$. These capacitors were fabricated from eight cells, each having approximately 20 $\mathrm{cm}^{2}$ of surface area that were connected in series to form the device. Each individual cell had a nominal rating of $16 \mathrm{~F}\left(0.8 \mathrm{~F} / \mathrm{cm}^{2}\right)$ at $3 \mathrm{~V}$. The $24 \mathrm{~V}, 8$-cell unpackaged capacitors attained specific energy levels of $5 \mathrm{~W} \cdot \mathrm{h} / \mathrm{kg}$ at specific powers as high as $1 \mathrm{~kW} / \mathrm{kg}$. The packaged $24 \mathrm{~V}$ devices suffered some performance loss and fell slightly below the unpackaged device levels achieving specific energy levels of approximately $4.5 \mathrm{~W} \cdot \mathrm{h} / \mathrm{kg}$ at a specific power of $500 \mathrm{~W} / \mathrm{kg}$. The constant-power discharge behavior indicated a significant loss of available energy when the temperature was lowered to $-24^{\circ} \mathrm{C}$. The equivalent series resistance of these capacitors, determined from the constant-current tests, was measured to be approximately 0.4 to $0.6 \mathrm{ohm}$ $\left(1\right.$ to $\left.1.5 \mathrm{ohm}-\mathrm{cm}^{2}\right)$ at room temperature $\left(25^{\circ} \mathrm{C}\right)$. This value did not change appreciably when tests were performed at $60^{\circ} \mathrm{C}$. When the temperature was lowered to $-20^{\circ} \mathrm{C}$, the resistance increased substantially to approximately 1.5 to $2.0 \mathrm{ohm}\left(3.75\right.$ to $\left.5 \mathrm{ohm}-\mathrm{cm}^{2}\right)$.

The self-discharge test data for these capacitors showed a significant increase in the selfdischarge at higher temperatures $\left(60^{\circ} \mathrm{C}\right)$. Round-trip efficiency for these capacitors, as measured by the constant-current charge and discharge tests, was approximately $85 \%$. 
Life-cycle tests were also conducted on these capacitors. The capacity fell below $80 \%$ of the initial capacity after 110,000 constant-current test cycles. A constant-current test current of $1 \mathrm{~A}$ was used so as to achieve a discharge time of about 18 to 20 seconds. It should be noted that a significant detrimental effect on the life-cycle performance of the capacitors was observed when the voltage was clamped for 10 seconds at the end of the charge and discharge portions of the test cycle.

The specific energy and specific power of all three sets of capacitors were well below those set by the near-term (1997) goals set by PNGV [1,2] of power/energy $>100$, specific energy $=6 \mathrm{~W} \cdot \mathrm{h} / \mathrm{kg}$ and energy density $=9 \mathrm{~W} \cdot \mathrm{h} / \mathrm{L}$, as well as the long-term (2006) PNGV goals of power/energy $>100$, specific energy $=18 \mathrm{~W} \cdot \mathrm{h} / \mathrm{kg}$ and energy density $=24 \mathrm{~W} \cdot \mathrm{h} / \mathrm{L}$. 


\section{BACKGROUND}

The electrochemical capacitor devices described in this report were deliverables from the U. S. Department of Energy - Idaho Operations Office (DOE-ID) Contract No. DE-AC0792ID13404 as part of the U.S. Department of Energy's (DOE) High Power Energy Storage Program. The Idaho National Engineering and Environmental Laboratory (INEEL) has responsibility for technical management, testing, and evaluation of high-power batteries and electrochemical capacitors under this Program. The DOE has developed various electrochemical capacitors as candidate power assist devices for the Partnership for a New Generation of Vehicles (PNGV) fast response engine requirement. This contract with Maxwell Energy Products, Inc. (Maxwell) was intended to develop a high-energy-density, high-power-density ultracapacitor that is capable of load leveling batteries in electric vehicles. The performance criteria for this device are delivery of $5 \mathrm{~W} \cdot \mathrm{h} / \mathrm{kg}$ of useful energy that can be used by the vehicle at an average power rating of $600 \mathrm{~W} / \mathrm{kg}$. The capacitor must also have an overall charge/discharge efficiency of $90 \%$, and a useful life of more than 100,000 discharge cycles. The deliverables reported on here are those prepared by Maxwell Energy Products, Inc. at various stages of their developmental program. Deliverables were sent to the INEEL's Energy Storage Technologies (EST) Laboratory for independent testing and evaluation. This report describes performance testing on three sets of capacitors delivered over a two year period. Additional testing has been done on Set \#2 described herein, as well as on an additional deliverable from Maxwell. These test results will be documented in a follow-up report. 


\section{PURPOSE}

The purpose of this report is to present the electrical performance for three sets of electrochemical capacitors supplied by Maxwell Energy Products, Inc. that constitute various phases of their capacitor development efforts. All tests were conducted in the Energy Storage Testing (EST) Laboratory which is part of the Automotive Systems and Technology (AS\&T) Department at the Idaho National Energy and Environmental Laboratory (INEEL). 


\section{DESCRIPTION OF CAPACITOR SETS}

The capacitor sets had the following specifications as supplied by the researchers at Maxwell Energy Products, Inc.

\section{Set \#1 (three capacitors; serial numbers \#1141, \#1142 and \#1143) received July 1996}

Figure 1 is a photograph of capacitor \#1143 that is physically the same as capacitors \#1141 and \#1142. Maxwell Energy Products, Inc. provided the information shown in Table 1 regarding the three capacitors received for testing. The voltage rating for each capacitor was given as $3.0 \mathrm{~V}$. Each capacitor will be referred to by its serial number in the discussions regarding the measured test performance of the individual capacitors.

General fabrication information:

The capacitors were made using proprietary materials and assembly procedures; however, a general description has been given by Maxwell: "These capacitors were made using a sintered metal/carbon fiber composite electrode. This composite electrode was used by Maxwell as it offers high capacitance, low resistivity, extremely large specific surface area (1000-2500 $\left.\mathrm{m}^{2} / \mathrm{g}\right)$, a robust structure and is chemically inert. An advanced non-aqueous electrolyte was used that provides higher operation voltage that leads to higher energy and power density and an optimized conductivity that reduces the internal resistance." The weight of each capacitor as measured prior to testing at the INEEL was $33.20 \mathrm{~g}(0.03320 \mathrm{~kg})$ for $\# 1141,33.23 \mathrm{~g}(0.03323 \mathrm{~kg})$ for $\# 1142$, and $31.90 \mathrm{~g}(0.03190 \mathrm{~kg})$ for \#1143. 


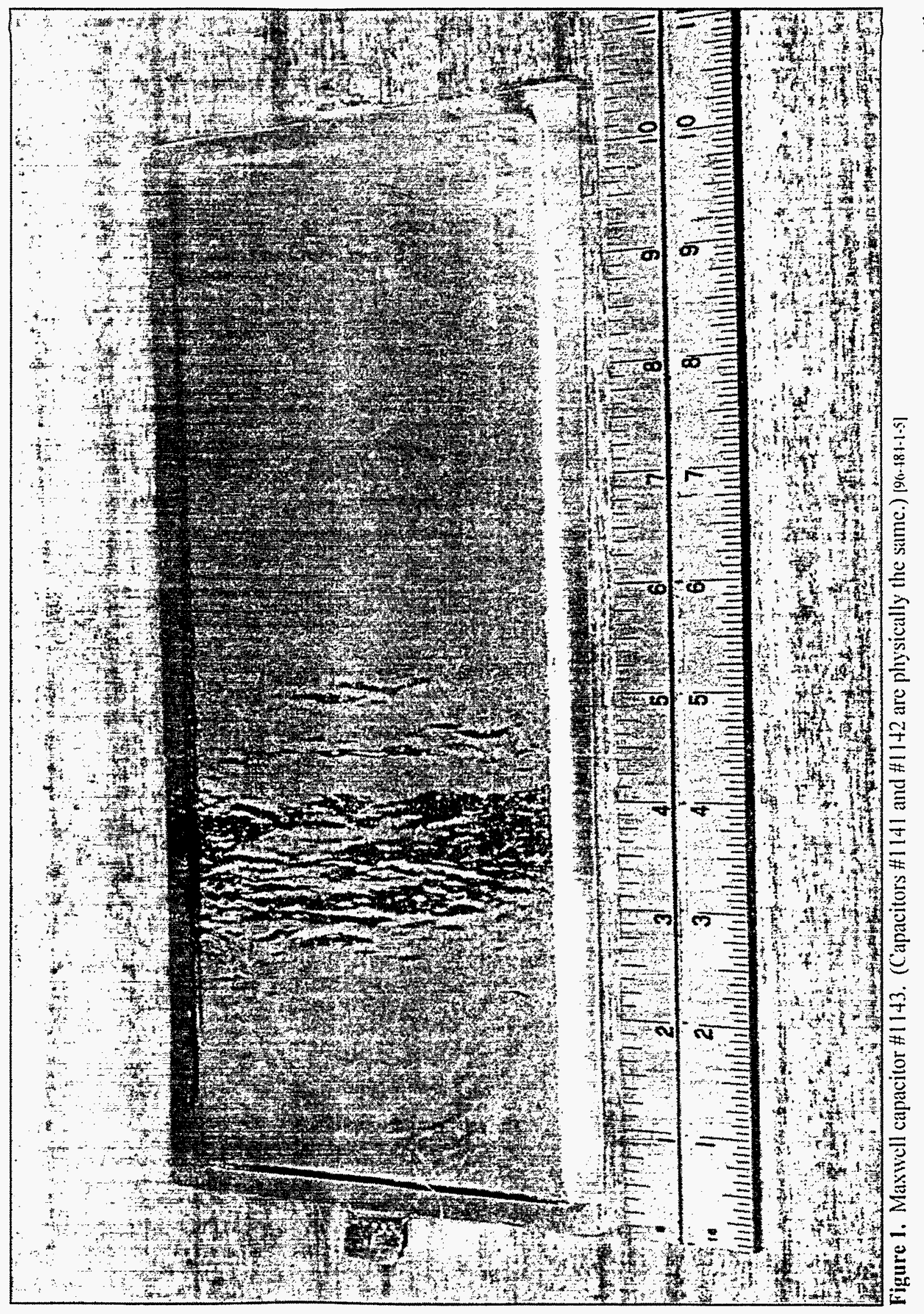


Table 1. Data supplied by Maxwell Energy Products, Inc. for testing of capacitors of Set \#1.

\begin{tabular}{|l|c|c|}
\hline \multicolumn{1}{|c|}{ Sample ID } & Weight $(\mathbf{g})$ & Volume $(\mathbf{m l})$ \\
\hline $200-1141$ & 33.27 & 32.80 \\
\hline $200-1142$ & 33.31 & 32.80 \\
\hline $200-1143$ & 32.00 & 31.56 \\
\hline
\end{tabular}

\begin{tabular}{|c|c|c|c|c|c|c|}
\hline Sample ID & $C_{a}(\mathbf{F})$ & $C_{b}(f)$ & $\mathbf{R}_{\mathrm{e}}(\mathrm{ohm})$ & $\mathbf{R}_{\mathrm{d}}$ (ohm) & E (grav) & $E$ (vol) \\
\hline $200-1141$ & 165.0 & 156.8 & 0.0093 & 0.0099 & 6.2 & 6.3 \\
\hline $200-1142$ & 155.7 & 147.5 & 0.0095 & 0.0090 & 5.8 & 5.9 \\
\hline $200-1143$ & 154.9 & 145.5 & 0.0096 & 0.0091 & 6.1 & 6.1 \\
\hline \multicolumn{7}{|c|}{$\begin{array}{l}\text { a. Capacitance taken from } \mathrm{V}_{\mathrm{w}} \text { to } 1 / 2 \mathrm{~V}_{\mathrm{w}}\left(\mathrm{V}_{\mathrm{w}}=3 \mathrm{~V}\right) \\
\text { b. Capacitance taken from full discharge } \\
\text { c. Resistance measurement for constant-current test } \\
\text { d. Resistance measurement for } \mathrm{AC} \text { impedance at } 1 \mathrm{~Hz}\end{array}$} \\
\hline
\end{tabular}

\section{TESTS CONDUCTED ON SET \#1 [3]}

1. General Test Instructions

- Charge at $1 \mathrm{~A}$ constant current unless specified otherwise.

- Clamp voltage for 20 seconds at the charge and discharge voltage endpoints specified on each cycle.

- Complete three test cycles at each test condition.

2. Constant-Current Tests

- Voltage limits are $0.05 \mathrm{~V}$ to $3.0 \mathrm{~V}$.

- $\quad$ Begin discharging each capacitor at constant currents of $2,4,8,16,24$ and 32 amperes (A) or until a discharge time of approximately 10 seconds is attained. 
3. Constant-Power Tests

- Voltage limits are $1.5 \mathrm{~V}$ to $3.0 \mathrm{~V}$.

- Discharge each capacitor at a constant power of 4.5, 9.0, 18.0,36.0, 54.0 and 72.0 watts $(\mathrm{W})$ or until a discharge time of approximately 10 seconds is attained.

4. Leakage-Current Test

- Charge capacitor to $3.0 \mathrm{~V}$ and clamp voltage. Monitor current for 24 hours or until the leakage current attains a stable value.

5. Self-Discharge Test

- Charge capacitor to $3.0 \mathrm{~V}$ and measure voltage for 48 hours under open-circuit conditions.

\section{Set \#2 (three capacitors; serial numbers \#1021, \#1022 and \#1023 received September 1996) [3]}

General fabrication information:

Figure 2 is the $3 \mathrm{~V}, 2300 \mathrm{~F}$ capacitor (\#1021) that is physically the same as \#1022 and \#1023. Maxwell Energy Products, Inc. [4] provided the following information concerning the construction of these capacitors: "These capacitors consisted of two porous electrodes isolated from electrical contact by a porous separator. Both the separator and the electrodes were impregnated with an organic-based electrolytic solution. The electrodes used in these capacitors were made from a composite of activated carbon and aluminum. The carbon has an extremely high volumetric surface area that is responsible for the high capacitance of the electrodes. The aluminum matrix is added as a means of reducing the number of carbon/carbon contact points the current must flow through before it reaches the current collecting phase. This decrease in the number of contact points lowers the internal resistance of the capacitor. The aluminum composite structure also minimizes the packaging pressure required to achieve the lowest internal resistance in the device. The improved packaging increases the energy density of the capacitor by minimizing the weight of the device. These large-scale capacitors were constructed by connecting many single cells in parallel in one individual cell housing. The capacitor housings were constructed of aluminum and had overall dimensions of $13.5 \mathrm{~cm} \times 6 \mathrm{~cm} \times 5.7 \mathrm{~cm}$ ( $462 \mathrm{~cm}^{3} ; 0.462$ liter). The case consists of two cells, each side acting as a terminal for current collection."

The capacitors were rated at $3 \mathrm{~V}$ and $2300 \mathrm{~F}$ with a maximum charge and discharge current of $300 \mathrm{~A}$. The weights of the individual capacitors prior to the start of testing at the INEEL were: $622 \mathrm{~g}(0.622 \mathrm{~kg})$ for \#1021; $633 \mathrm{~g}(0.633 \mathrm{~kg})$ for \#1022; and $635 \mathrm{~g}(0.635 \mathrm{~kg})$ for $\# 1023$. 


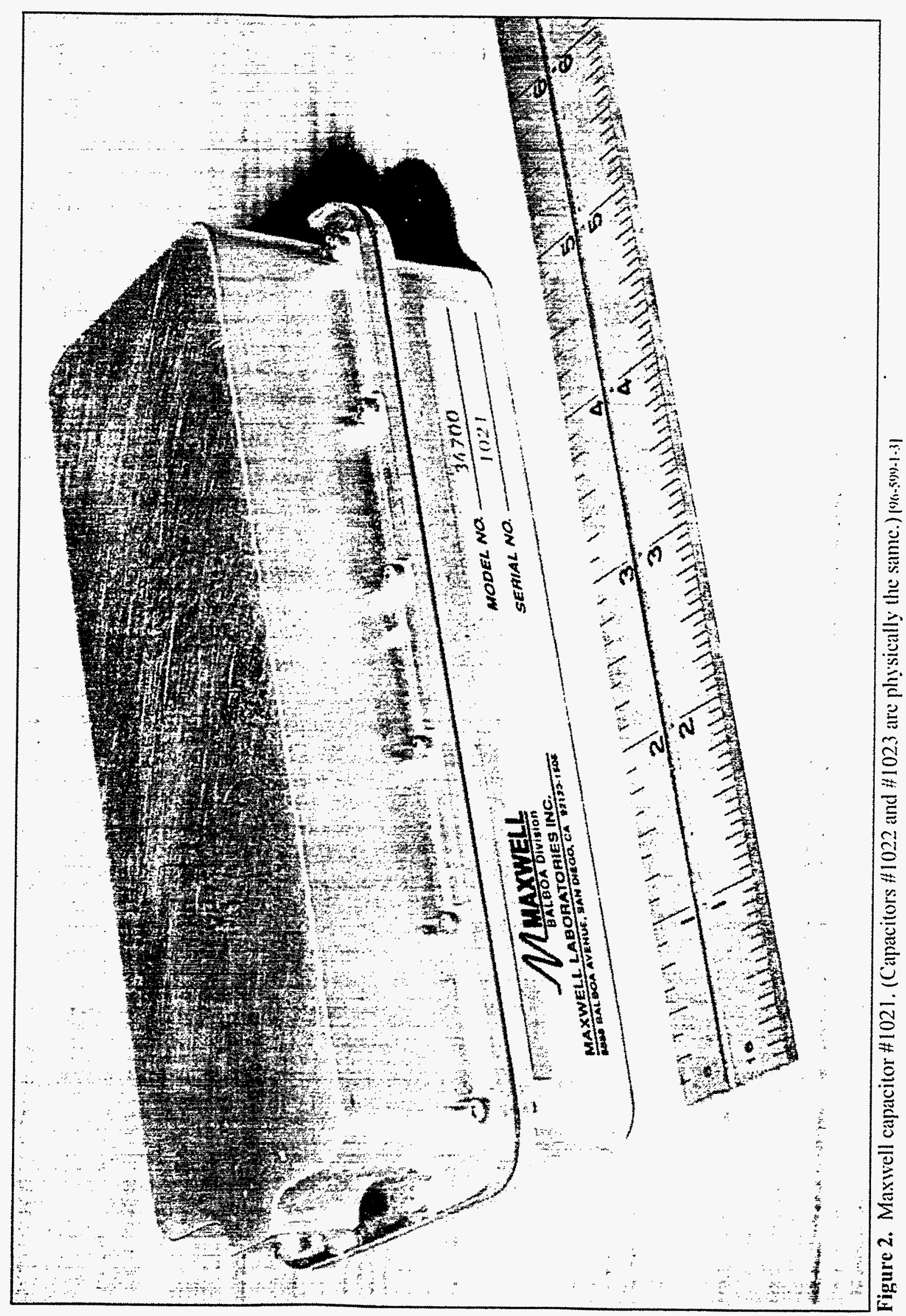




\section{TESTS CONDUCTED ON SET \#2 $2^{[3]}$}

1. General Test Instructions

- A special test fixture for holding the capacitors and making electrical contact with the current collectors was supplied by Maxwell Energy Products, Inc. for use in the tests.

- Clamp the voltage for 10 seconds at the charge and discharge voltage endpoints specified on each test cycle.

- $\quad$ Complete three cycles for the constant-current and constant-power tests.

2. Constant-Current Tests

- Voltage limits are $0.05 \mathrm{~V}$ to $3.0 \mathrm{~V}$.

- Use constant current charge and discharge currents of 10,20,50,100,200,250 and $300 \mathrm{~A}$. The constant current discharge value can be increased or decreased until a discharge time of approximately 10 seconds is attained; at this point the test should be terminated.

3. Constant-Power Tests

- Voltage limits are $1.5 \mathrm{~V}$ to $3.0 \mathrm{~V}$.

- The charging current to be used for these test is $10 \mathrm{~A}$.

- Discharge each capacitor at constant-powers of 10, 20, 50, 100, 200, 250 and 300 watts. The constant power discharge used in this tests can be decreased or increased compared to those listed above in order to achieve a measured discharge time of approximately 10 seconds, at which point the test should be terminated.

4. Leakage-Current Test

- Charge capacitor to $3.0 \mathrm{~V}$ and clamp voltage. Monitor current for 24 hours or until the leakage-current has stabilized.

5. Self-Discharge Test

- Charge capacitor to $3.0 \mathrm{~V}$ and measure voltage for 48 hours under open-circuit conditions. 
6. Life-Cycle Test

- Voltage limits are $1.5 \mathrm{~V}$ to $3.0 \mathrm{~V}$.

- Condition the capacitor at $25 \pm 3^{\circ} \mathrm{C}$ until thermal equilibrium is reached. Initialize the voltage on the capacitor at $1.5 \mathrm{~V}$. Then charge the capacitor at a constant current that corresponds to that required to raise the voltage on the capacitor to $3.0 \mathrm{~V}$ in $18( \pm 1)$ seconds. (The appropriate constant current to use is to be determined from the constant-current tests.) Maintain $3.0 \mathrm{~V}$ on the capacitor for $10( \pm 0.5)$ seconds. Then discharge the capacitor to $1.5 \mathrm{~V}$ at the same constant current used to charge the capacitor. Maintain $1.5 \mathrm{~V}$ on the capacitor for 10 $( \pm 0.5)$ seconds. This defines one test cycle. Repeat this cycle throughout the testing, adjusting the current as needed in order to maintain the initial charge/discharge time of $18( \pm 1)$ seconds. This test cycle results in a cycle time of approximately 56 seconds.

- Acquire data for the first ten cycles and then every 500 cycles thereafter.

- Check data files each day to determine cycle time. Adjust the constant current charge/discharge value to maintain a 56 second cycle to within $5 \%$ (approximately 3 seconds).

- Terminate the test when the watthour value has decreased to $80 \%$ of its initial value. (From the data supplied by Maxwell, the initial watt hour value should be $2.16 \mathrm{~W} \cdot \mathrm{h}$ for these capacitors assuming a $3 \mathrm{~V}$ to $1.5 \mathrm{~V}$ charge/discharge range and a capacitance of $2300 \mathrm{~F}$.)

7. Temperature Tests

- For the temperature tests the capacitors were placed in a temperature controlled chamber controllable to $+/-2^{\circ} \mathrm{C}$.

- Temperature tests are to be conducted at $-20^{\circ} \mathrm{C}, 23^{\circ} \mathrm{C}$ and $55^{\circ} \mathrm{C}$.

\section{Set \#3 (several capacitors) received December 1995}

These capacitors were eight-cell, $24 \mathrm{~V}$ packaged and unpackaged devices (see Figures 3 and 4). They were rated at $2 \mathrm{~F}$. These capacitors were fabricated from eight cells, each having approximately $20 \mathrm{~cm}^{2}$ of surface area that were connected in series to form the device. Each individual cell had a nominal rating of $16 \mathrm{~F}\left(0.8 \mathrm{~F} / \mathrm{cm}^{2}\right)$ at $3 \mathrm{~V}$. The electrodes are based on carbon and the electrolyte contains mixtures of organic liquids containing a conductive additive. 


\section{TESTS CONDUCTED ON SET $\# 3^{[3]}$}

1. General test instructions

- Charge at $1 \mathrm{~A}$ constant current unless otherwise specified.

- Clamp the voltage for 20 seconds at the charge and discharge voltage endpoints specified on each test cycle.

- Complete three test cycles at each test condition.

2. Constant-Current Tests

- Voltage limits were $1.0 \mathrm{~V}$ to $23.0 \mathrm{~V}$.

- Discharge the capacitor at constant currents of $0.25 \mathrm{~A}, 0.5 \mathrm{~A}, 1 \mathrm{~A}, 2 \mathrm{~A}, 4 \mathrm{~A}, 8 \mathrm{~A}$ and 10 A.

- Charge the capacitor at $1 \mathrm{~A}$ for all tests except where the discharge currents are $0.25 \mathrm{~A}$ and $0.5 \mathrm{~A}$. In these tests charge at 0.25 and $0.5 \mathrm{~A}$ respectively.

3. Constant-Power Tests

- Discharge voltage limits are $1.0 \mathrm{~V}$ to $23.0 \mathrm{~V}$.

- Discharge each capacitor at constant-powers of $0.375 \mathrm{~W}, 0.75 \mathrm{~W}, 1.5 \mathrm{~W}, 3 \mathrm{~W}, 6 \mathrm{~W}, 9 \mathrm{~W}$ and $12 \mathrm{~W}$. (Note: Use $1.5 \mathrm{~V}$ for the discharge voltage cut off for the $12 \mathrm{~W}$ test.)

4. Self-Discharge Test

- Charge capacitor to $23 \mathrm{~V}$ and measure voltage for 48 hours under open-circuit conditions.

5. Life-Cycle Test

- Voltage limits are $1.5 \mathrm{~V}$ to $23.0 \mathrm{~V}$.

- Cycle is to be $1 \mathrm{~A}$ charge and $1 \mathrm{~A}$ discharge.

- Take data for the first ten cycles and then every 500 cycles thereafter.

- Check data files daily to determine cycle time.

- This test is to be conducted at approximately $23^{\circ} \mathrm{C}$.

6. Temperature Tests

- For the temperature tests, the capacitors were placed in a temperature controlled chamber controllable to $+/-2^{\circ} \mathrm{C}$. 

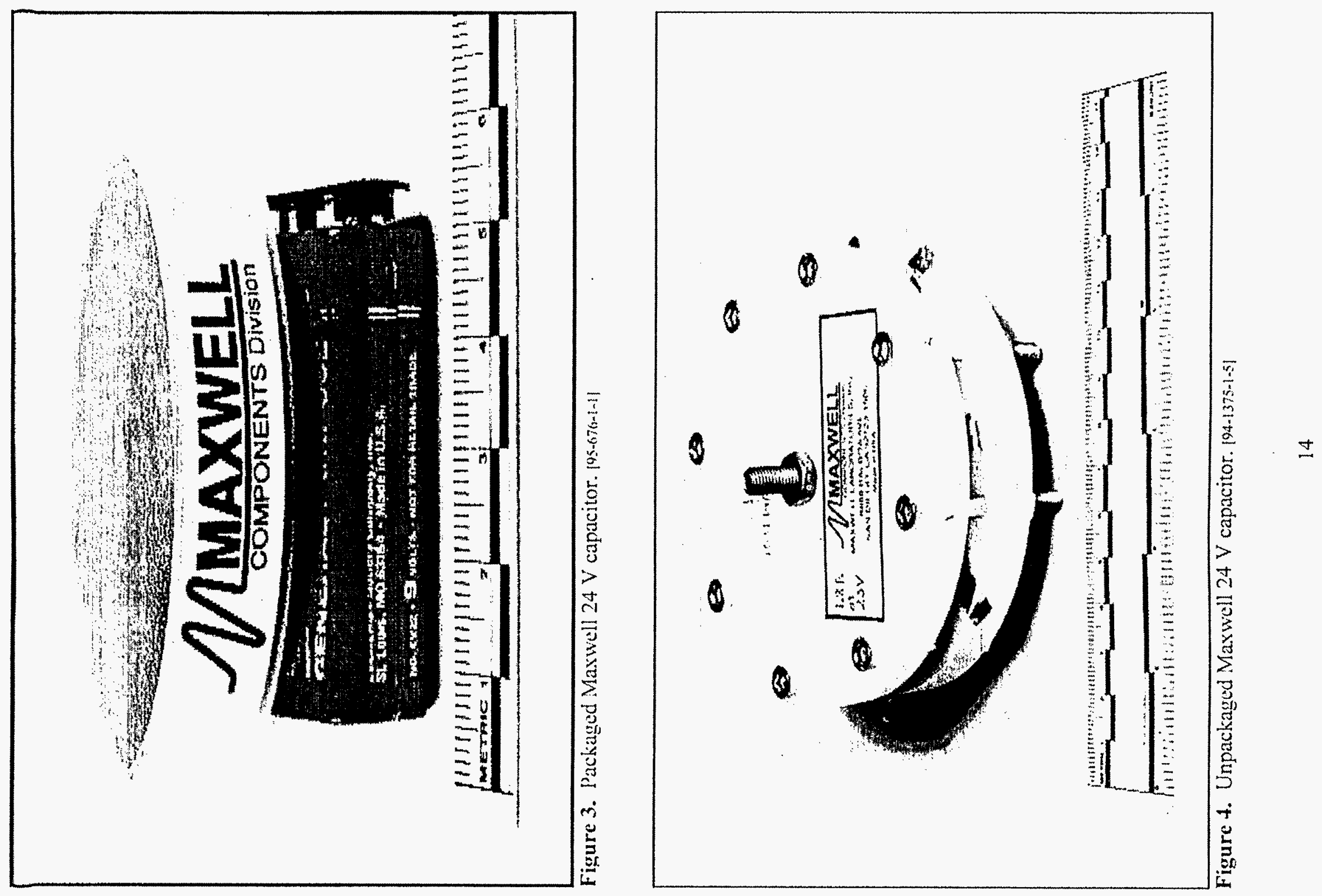


\section{TEST RESULTS}

\section{Set \#1 (Capacitors \#1141, \#1142 and \#1143) [5-7]}

The measured as-tested weights for the three capacitors were:

$\begin{array}{ll}\text { \#1141: } & 33.20 \mathrm{~g}(0.03320 \mathrm{~kg}) \\ \text { \#1142: } & 33.23 \mathrm{~g}(0.03323 \mathrm{~kg}) \\ \text { \#1143: } & 31.90 \mathrm{~g}(0.03190 \mathrm{~kg})\end{array}$

\section{Constant-Current Test Results}

The first test that was run on these capacitors was the constant-current test. In Figure A-1 is shown a representative three-cycle constant-current test on capacitor \#1143 that used a $1 \mathrm{~A}$ constant-current charge from $0.05 \mathrm{~V}$ to $3 \mathrm{~V}$ and a constant-current discharge from $3 \mathrm{~V}$ to $0.5 \mathrm{~V}$. Shown in this figure is the capacitor voltage (in volts) as a function of test time (in minutes). The voltage can be observed to increase fairly linearly with time during the charging portion of the test cycle as predicted by the ideal-capacitor model expressed by equation (3) in Appendix B. The charging step is followed by a 20 -second hold at $3 \mathrm{~V}$ at which point a $16 \mathrm{~A}$ constant-current discharge was begun. The voltage is seen to decrease from $3 \mathrm{~V}$ to $0.05 \mathrm{~V}$ in a fairly linear manner with time as would be expected based on the ideal-capacitor model, equation (4). The voltage is held at the $0.05 \mathrm{~V}$ level for 20 seconds before the start of the next cycle of the test. The test current corresponding to the test cycle data shown in Figure A-1 is shown in Figure A-2. The constant-current charge ( $1 \mathrm{~A})$, voltage hold (20 seconds) and constant-current discharge (16 A) portions of the test cycle can be seen. To further examine the degree to which these capacitors follow the theoretical ideal-capacitor model, in Figure A-3(a) are shown the time dependence of the experimental charge and discharge voltage data and the calculated charge and discharge voltage using equations (3) and (4) for capacitor \#1143. The values for C (140.7 F) and $R_{s}$ (11.5 milliohms) used in the calculated curves were the experimentally derived values from the $2 \mathrm{~A}$ discharge data and the constant-current test values $\left(I_{c}=1 \mathrm{~A}\right.$ and $\left.I_{d}=2 \mathrm{~A}\right)$ were the test values. Deviations from the ideal-capacitor model can be seen in both the charge and discharge portions of the test cycle. During charging, the capacitor voltage increases faster (larger slope) at the shorter times ( $<90$ seconds; $<0.8 \mathrm{~V})$ than the model implying that the capacitance is smaller than $140.7 \mathrm{~F}$. At times greater than approximately 110 seconds $(>1.1 \mathrm{~V})$ the voltage increases slower than that predicted by the ideal-model (smaller slope) indicating that the capacitance is greater than the $140.7 \mathrm{~F}$ used in the model. The deviations of the discharge voltage data from the ideal-model also indicate that at voltages greater than $0.8 \mathrm{~V}$ the capacitance is larger than $140.7 \mathrm{~F}$, and at voltages less than $0.8 \mathrm{~V}$ the capacitance is smaller than this value. These observed voltage deviations from the ideal-model can be more clearly seen in Figure A3(b) where the potential difference (experimental-theory) values are plotted as a function of the measured test voltage. The deviations can be attributed to the changing capacitance as a function of the capacitor potential as previously described.

Initially, the constant-current tests were done using a $1.5 \mathrm{~V}$ to $3.0 \mathrm{~V}$ charge and discharge voltage range. Due to the deviations from the ideal-capacitor model noted above, the constantcurrent test using a $0.05 \mathrm{~V}$ to $3.0 \mathrm{~V}$ charge and discharge voltage range was done on 
capacitor \#1143 to determine if the voltage range affected the measured capacitance value (see below). The experimental constant-current discharge voltage data (cycle two of the three test cycles) as a function of the discharge time (seconds) for the various constant-current discharge currents used in the tests for capacitors $\# 1141$ and \#1142 (for a discharge voltage range of $3.0 \mathrm{~V}$ to $1.5 \mathrm{~V}$ ), and \#1143 (for a discharge voltage range of $3.0 \mathrm{~V}$ to $0.05 \mathrm{~V}$ ) are shown in Figures A-4, A-5 and A-6 respectively. The constant-current charging current was 1 A for all of these tests. The average equivalent series resistance, ESR, values (average value from the three test cycles) calculated using equation (73) as a function of the constant-current discharge current are shown in Figure A-7. The ESR values for the three capacitors ranged between approximately 9 to 22 milliohms and generally increased with increasing discharge current. Equation (64) was used to calculate the capacitances of the three devices as a function of the constant-current discharge current. The values are shown in Figure A-8. The values are seen to vary from $150 \mathrm{~F}$ to $163 \mathrm{~F}$ at $2 \mathrm{~A}$ decreasing to $95 \mathrm{~F}$ to $135 \mathrm{~F}$ at $16 \mathrm{~A}$. The capacitance of each capacitor decreased as the discharge current increased. As pointed out above, the constant-current test was repeated using a discharge voltage range of $3.0 \mathrm{~V}$ to $0.05 \mathrm{~V}$ for device \#1143. These capacitance values are also plotted in Figure A-8. There was indeed a difference in the measured capacitance that can be attributed to the non-ideal capacitor behavior of the device that is not accounted for when using equation (64) to calculate the capacitance from the constant-current discharge (or charge) test data.

\section{Constant-Power Test Results}

The constant-power test was the next test to be conducted on the three capacitors. Shown in Figure A-9 is the test voltage as a function of test time for capacitor \#1143 for a three-cycle constant-power test that used a 1 A constant-current charge from $1.5 \mathrm{~V}$ to $3.0 \mathrm{~V}$, followed by a 20 seconds hold at $3.0 \mathrm{~V}$, followed by an $18 \mathrm{~W}$ constant-power discharge from $3.0 \mathrm{~V}$ to $1.5 \mathrm{~V}$ and, finally, a 20 second hold at $1.5 \mathrm{~V}$. The corresponding test current for this three-cycle test is shown in Figure A-10. The measured constant-power discharge voltage as a function of discharge time for various constant-power discharges is shown in Figures A-11, A-12 and A-13 for capacitors \#1141, \#1142 and \#1143 respectively. Using equation (74), the specific energy as a function of specific power was calculated from the constant-power discharge data. The results are shown in Figure A-14(a) (as a linear-linear plot) and in Figure A-14(b) (as a log-log plot). The specific energy for all three devices was approximately $4.3 \mathrm{Wh} / \mathrm{kg}$ at $141 \mathrm{~W} / \mathrm{kg}$, decreasing to approximately $1.9 \mathrm{Wh} / \mathrm{kg}$ at $1100 \mathrm{~W} / \mathrm{kg}$ (for capacitors \#1142 and \#1143). The specific energy of capacitor \#1141 was observed to decrease more rapidly with increasing specific power (it was only tested to an $18 \mathrm{~W}$ constant-power discharge; see Figure A-11).

\section{Leakage-Current Test Results}

The leakage-current test was then conducted on capacitors \#1142 and \#1143. The measured leakage-current (amperes) to maintain a potential of $3 \mathrm{~V}$ on the capacitors as a function of test time (hours) is shown in Figure A-15. It took approximately 20 hours for the leakagecurrent to stabilize at approximately 500 microamps for both capacitors. Capacitor \#1143 had a slightly better (i.e., lower) leakage-current. Equation (77) was used to calculate the time dependence of the equivalent parallel resistance (EPR) of the capacitors. These data are shown in Figure A-16 for both capacitors. The stable EPR of both devices after approximately 20 hours 
was between 700 and $1000 \mathrm{ohms}$. The noise in the curves is do to the noise in the Maccor current measuring module that was used to conduct the tests.

\section{Self-Discharge Test Results}

The self-discharge test was the next test to be run on the three capacitors. In Figure A-17 are shown the self-discharge potential (volts) as a function of test time (hours) for the three capacitors of Set \#1. Capacitor \#1142 was the best of the three devices as it had the smallest decrease in voltage over the 72-hour test time. Capacitor \#1143 was next best, followed by \#1141. From these data the self-discharge energy loss factor, equation (78), was calculated for the three capacitors as a function of test time. The results are shown in Figure A-18. Capacitor \#1142 was the best device losing approximately $43 \%$ (after 72 hours) of its initial stored energy due to non-ideal energy storage/transfer processes. Device \#1143 was next best losing $\sim 57 \%$ of its stored energy after 72 hours. Capacitor \#1141 lost $\sim 72 \%$ of its initial stored energy after 72 hours. For those applications where a capacitor needs to supply energy (and power) at times after its initial charging, the self-discharge energy loss factor is an important measure of a capacitor's performance. These data show that a considerable voltage decrease can occur in a capacitor due to self-discharge processes over a relatively short ( 3 days) period of time which can dramatically lower its stored energy.

\section{Set \#2 (Capacitors \#1021, \#1022 and \#1023) [5-7]}

The measured, as-tested weights of the three capacitors were:

$$
\begin{array}{ll}
\# 1021: & 622 \mathrm{~g}(0.622 \mathrm{~kg}) \\
\# 1022: & 633 \mathrm{~g}(0.633 \mathrm{~kg}) \\
\# 1023: & 635 \mathrm{~g}(0.635 \mathrm{~kg})
\end{array}
$$

\section{Constant-Current Test Results}

ESR and Capacitance Measurements. A representative constant-current test cycle is shown in Figure A-19 for capacitor \#1022 (a series of three cycles was used during the tests) that used a 100 A constant-current charge to $3.0 \mathrm{~V}$ followed by a 20 -second hold at $3.0 \mathrm{~V}$, and then a 100 A constant-current discharge to $0.01 \mathrm{~V}$ followed by a 20 -second hold at this voltage before the start of the next test cycle. From these data can be seen the IR-induced increase at the start of the charge, and a drop in the voltage at the start of the discharge portions of the test cycle due to the equivalent series resistance of the capacitor. The voltage is also seen not to be a linear function of time [as predicted by the ideal-capacitor model, equations (3) and (4)] for either the charge or discharge portions of the test cycle. The corresponding test current measured during the constant-current test cycle shown in Figure A-19 is shown in Figure A-20. The $100 \mathrm{~A}$ constant-current charge and discharge portions of the test can be seen as well as the current decline during the 20 -second hold at $3.0 \mathrm{~V}$ and at $0.01 \mathrm{~V}$. In order better to observe the voltage deviations from the ideal-capacitor model during charging and discharging of these capacitors, Figures A-21 and A-22 compare the test cycle-\#2 experimental voltage and the voltage calculated using the theoretical ideal-capacitor model, equations (3) and (4), as a function of test 
time. The calculations are shown for the charge and discharge portions of the constant-current test cycle for a $10 \mathrm{~A}$ test (Figure A-21) and for a $150 \mathrm{~A}$ test (Figure A-22) for capacitor \#1021. For the $10 \mathrm{~A}$ test (Figure A-21), the experimental voltage is larger than that predicted by the model except at the $0.01 \mathrm{~V}$ and $3.0 \mathrm{~V}$ end points of the test [values of $\mathrm{R}=0.50 \mathrm{milliohms}$ and $\mathrm{C}$ $=2384.5 \mathrm{~F}$ were used in equations (3) and (4)]. These deviations can probably be attributed to a capacitance that depends on the voltage on the capacitor as the slope of the experimental data is smaller (i.e., larger capacitance than that used in the model) at voltages greater than $\sim 1.5 \mathrm{~V}$. Similarly, the slope is greater (i.e., smaller capacitance than that used in the model) for voltages less than $\sim 1.5 \mathrm{~V}$. The comparisons shown in Figure A-22 for the $150 \mathrm{~A}$ test also indicate that the capacitance of the capacitor is not a constant, but varies with the voltage on the capacitor. The values of $R=0.18$ milliohms and $C=2330.0 \mathrm{~F}$ were used in the model for the $150 \mathrm{~A}$ test.

Initially, the constant-current tests were run using a $1.5 \mathrm{~V}$ to $3.0 \mathrm{~V}$ voltage range but were then redone using a $0.01 \mathrm{~V}$ to $3.0 \mathrm{~V}$ voltage range. For these tests the three capacitors were tested at various constant-currents from $10 \mathrm{~A}$ to $100 \mathrm{~A}$ using a Maccor test system, and from $100 \mathrm{~A}$ to $300 \mathrm{~A}$ (the recommended upper current limit suggested by Maxwell) using a Bitrode test system. Shown in Figures A-23, A-24 and A-25 are the constant-current discharge test data for capacitor \#1021 using a charging and discharging voltage range of $1.5 \mathrm{~V}$ to $3.0 \mathrm{~V}$ and $0.01 \mathrm{~V}$ to $3.0 \mathrm{~V}$. Figures A-26, A-27 and A-28 display the constant-current discharge test data for capacitor \#1022 using both of the voltage test ranges. Figures A-29, A-30 and A-31 are the test data for capacitor \#1023, also using both of the voltage test ranges. These test data were from cycle-\#2 of the three-cycle test sequence and were taken at a nominal capacitor temperature of $23^{\circ} \mathrm{C}$. From the constant-current test data the equivalent series resistance (ESR) and capacitance values as a function of the constant current test current were calculated using equations (73) and (65) respectively. In Figure A-32 are plotted the ESR values for capacitor \#1021 as a function of the discharge test current for the constant-current tests conducted using a Maccor tester over a discharge voltage range of $3 \mathrm{~V}$ to $1.5 \mathrm{~V}$ (Test \#1) and for the tests conducted over a voltage range of $3 \mathrm{~V}$ to $0.01 \mathrm{~V}$ (Test \#2). The constant-current tests conducted using a Bitrode tester did not allow the calculation of the ESR values because the time increment between voltage readings was 0.1 second that is too large for accurate measurements when equation (73) is used. There was a large difference between the ESR values determined from Test \#1 and Test \#2. The only thing different between these tests (other than the discharge voltage range, which should not affect these measurements) is that the voltage hold between the charge and discharge portions of the test cycle was 10 seconds for Test $\# 1$ and 20 seconds for Test \#2. Test $\# 2$ was also done after constant-current tests using the Bitrode tester had been completed and also after the constantpower tests had been conducted. The time increment between voltage measurements was 0.030 second for all of the discharge tests, except for the $100 \mathrm{~A}$ discharge test where the time increment was 0.012 second. Perhaps something internal to this device had changed during the course of these tests causing its performance to change (for the better). The ESR values increased slightly with increasing constant-current discharge current, reached a maximum, and then decreased as the discharge current was further increased.

Similarly, the measured ESR values for capacitor \#1022 are shown in Figure A-33. For this device, the differences between the Test \#1 and Test \#2 values are not as great as that observed for capacitor \#1021. During the constant-current tests on this capacitor the constant voltage hold between the charge and discharge portions of the test cycle was 10 seconds; 10 
seconds was also used for Test $\# 2$ for the $10 \mathrm{~A}$ and $20 \mathrm{~A}$ tests, but 20 seconds for the $50 \mathrm{~A}$ and $100 \mathrm{~A}$ tests. The ESR values for capacitor \#1023 for Test $\# 1$ and Test $\# 2$ are shown in Figure A-34. A relatively small difference in the ESR values was observed between these two tests. The constant voltage hold times between the charge and discharge portions of the test cycle were the same as given previously for device \#1022. The ESR values for capacitors \#1022 and \#1023 generally tended to decrease in a nonlinear manner with increasing discharge current. This could be due, in part, to an increase in the internal temperature of the devices arising from the resistance heating at the larger test currents.

The measured capacitance of the three capacitors as a function of the discharge current is presented in Figures A-35, A-36 and A-37. The values presented are from those constant-current tests conducted using the Maccor tester $(10 \mathrm{~A}$ to $100 \mathrm{~A})$ and the Bitrode tester $(100 \mathrm{~A}$ to $300 \mathrm{~A})$ and using a voltage discharge range of $3 \mathrm{~V}$ to $1.5 \mathrm{~V}$, and $3 \mathrm{~V}$ to $0.01 \mathrm{~V}$ as labeled in the figures. The Bitrode tester had to be used for the higher current tests as it was the only tester channel available at the time of the test that was capable of going to $300 \mathrm{~A}$. There is an obvious discontinuity between the values at $100 \mathrm{~A}$ using the Maccor and Bitrode testers. As both testers were calibrated before their use, we do not have an explanation for this observation except to state that the precision of the Bitrode tester is not nearly as good as the Maccor tester and that the time increment between data collection is only 0.1 second for the Bitrode tester. There was also a large difference between the capacitance calculated from the $3 \mathrm{~V}$ to $1.5 \mathrm{~V}$ discharge range data as compared with that calculated from the $3 \mathrm{~V}$ to $0.01 \mathrm{~V}$ discharge range data. This discrepancy is due to the fact that the discharge voltage is not a linear function of the discharge time (as previously discussed) because the capacitance of the device is not a constant, but is a function of the voltage (charge) on the capacitor. The capacitance values calculated using the $3 \mathrm{~V}$ to $0.01 \mathrm{~V}$ discharge range are the most accurate when using equations (64) and (65) since the calculations include a greater range $(\Delta \mathrm{V}$ and $\Delta t)$ of the experimental discharge data. In general, the capacitance of the three capacitors decreased with increasing discharge current in a somewhat linear fashion. Using the $3 \mathrm{~V}$ to $0.01 \mathrm{~V}$ values at a $23^{\circ} \mathrm{C}$ test temperature, the average capacitance of the three capacitors was approximately $2400 \mathrm{~F}$ at a $10 \mathrm{~A}$ discharge, decreasing to approximately $2275 \mathrm{~F}$ at a discharge current of $300 \mathrm{~A}$. Capacitor \#1023 had the highest capacitance of the three devices.

Constant-current tests were also conducted at temperatures of $-20^{\circ} \mathrm{C}$ and $55^{\circ} \mathrm{C}$ on capacitor \#1022 in addition to the $23^{\circ} \mathrm{C}$ tests described above. Tests were also performed at $55^{\circ} \mathrm{C}$ on capacitors \#1021 and \#1023. All of these tests were done using various test channels on a Maccor tester. The discharge voltage as a function of discharge time for a series of constantcurrent tests at $10 \mathrm{~A}, 20 \mathrm{~A}, 50 \mathrm{~A}, 100 \mathrm{~A}$ and $200 \mathrm{~A}$ (the same constant-current was used to charge the capacitor) at a temperature of $55^{\circ} \mathrm{C}$ for capacitor \#1022 are shown in Figure A-38 over the discharge voltage range of $3 \mathrm{~V}$ to $1.5 \mathrm{~V}$. The results of the same tests $(10 \mathrm{~A}, 20 \mathrm{~A}, \quad 50 \mathrm{~A}, 100$ $\mathrm{A}, 200 \mathrm{~A}$ and $250 \mathrm{~A}$ ) at a test temperature of $-20^{\circ} \mathrm{C}$ over the discharge voltage range of $3 \mathrm{~V}$ to $0.05 \mathrm{~V}$ are shown in Figures $\mathrm{A}-39$ and $\mathrm{A}-40$. The discharge voltage is more nonlinear with time at $-20^{\circ} \mathrm{C}$ as compared to the $55^{\circ} \mathrm{C}$ and $23^{\circ} \mathrm{C}$ test results that can be observed upon close comparison of the data. ESR values were calculated using equation (73) from the temperature test data $\left(-20^{\circ} \mathrm{C}\right.$ and $55^{\circ} \mathrm{C}$ for capacitor \#1022, and $55^{\circ} \mathrm{C}$ for capacitors \#1021 and \#1023) and are shown in Figure $A-41$ as well as the previously discussed values measured from all three capacitors at $23^{\circ} \mathrm{C}$ that are shown in Figures A-32, A-33 and A-34. The ESR values were 
observed to be strongly influenced by the temperature of the capacitor, increasing at the lowest test temperature and generally decreasing as the temperature is increased. The ESR values were also observed to be highly nonlinear functions of the constant-current discharge current at $-20^{\circ} \mathrm{C}$ and $23^{\circ} \mathrm{C}$. At $-20^{\circ} \mathrm{C}$ and for a discharge current of $10 \mathrm{~A}$, the ESR value was $\sim 2.4$ milliohms, increasing to a maximum of $\sim 3.6$ milliohm at $100 \mathrm{~A}$, and then decreasing to $\sim 1.6$ milliohms at $250 \mathrm{~A}$. At $55^{\circ} \mathrm{C}$, the ESR values ranged from $\sim 0.45$ milliohms to $\sim 0.65$ milliohms at $10 \mathrm{~A}$, increasing to $\sim 0.6$ milliohms to $\sim 0.9$ milliohms at $100 \mathrm{~A}$, and decreasing slightly to $\sim 0.6$ milliohms to $\sim 0.8$ milliohms at the maximum test current of $200 \mathrm{~A}$. The nonlinearity with discharge current was not as pronounced at the $55^{\circ} \mathrm{C}$ test temperature.

The capacitance as a function constant-current discharge current at the $-20^{\circ} \mathrm{C}$ and $55^{\circ} \mathrm{C}$ test temperatures were also determined using equation (65) [which sums the ampere-hours (charge in coulombs) removed during the discharge divided by the voltage range of the discharge]. The measured values at $-20^{\circ} \mathrm{C}$ and $50^{\circ} \mathrm{C}$, in addition to those at $23^{\circ} \mathrm{C}$, are shown in Figure A-42 for those tests that used a $3 \mathrm{~V}$ to $1.5 \mathrm{~V}$ discharge range. In Figure A-43 are shown the capacitance values measured using equation (65) for those tests conducted at $-20^{\circ} \mathrm{C}(3 \mathrm{~V}$ to $0.05 \mathrm{~V}$ discharge range) and $23^{\circ} \mathrm{C}$ ( $3 \mathrm{~V}$ to $0.01 \mathrm{~V}$ discharge range). The capacitance values that are shown in these figures were those acquired using a Maccor tester; the capacitance values previously discussed that resulted from those tests that used the Bitrode tester at $23^{\circ} \mathrm{C}$ are not included in these figures due to the disparity of the measured values as discussed previously. A large difference occurs when one compares the capacitance values for tests where the discharge voltage was from $3 \mathrm{~V}$ to $1.5 \mathrm{~V}$ (Figure A-42) and for those tests where the discharge voltage was $3 \mathrm{~V}$ to $\sim 0.0 \mathrm{~V}$ (Figure A-43). For example, for capacitor \#1022 at a $10 \mathrm{~A}$ constant-current discharge for a $3 \mathrm{~V}$ to $1.5 \mathrm{~V}$ discharge range the capacitance was $2473.2 \mathrm{~F}$ as compared to $2300.9 \mathrm{~F}$ measured for the $3 \mathrm{~V}$ to $0.05 \mathrm{~V}$ test range. At a 200 A constant-current discharge the capacitance for the $3 \mathrm{~V}$ to $1.5 \mathrm{~V}$ discharge range was $200.7 \mathrm{~F}$, but for the $3 \mathrm{~V}$ to $0.05 \mathrm{~V}$ range it was determined to be $1216.7 \mathrm{~F}$. A very large difference indeed! It is becoming quite clear from the capacitor testing that has been performed at the INEEL that the notion of a constant value for the capacitance of a capacitor that is not a function of the voltage (and charge) on the capacitor is not correct and should be more closely examined when reporting the capacitance values of a capacitor. This point will be elaborated on further in a following section of this report where additional examples concerning this observation will be given. The capacitance values were generally the highest at $23^{\circ} \mathrm{C}$, decreasing slightly when the temperature was increased to $55^{\circ} \mathrm{C}$. When the temperature was decreased to $-20^{\circ} \mathrm{C}$, the capacitance values displayed a more significant decrease. At all of the test temperatures, the capacitance decreased in a fairly linear manner as the constant-current discharge current increased. The extent of the decrease with increasing discharge current was the greatest at $-20^{\circ} \mathrm{C}$, followed by those at $23^{\circ} \mathrm{C}$. The extent of decrease of the capacitance with increasing discharge current was the smallest at the $55^{\circ} \mathrm{C}$ test temperature.

RC-Time Constant Measurements. Knowing the values of the ESR and capacitance as a function of the constant-current discharge current allows the calculation of the RC-time constant [i.e., resistance (in ohms) times the capacitance (in farads) equals time (in seconds)] and its dependence on the discharge current for the capacitor (refer to Appendix B for further discussion). The RC-time constant is a measure of how fast the voltage and charge of the capacitor decrease when the capacitor is connected to an external load, or if the capacitor is 
short-circuited. In brief, a smaller RC-time constant allows the capacitor to charge and discharge faster. For example, during a constant voltage charge the RC-time constant specifies the time it takes the capacitor to charge to $63.2 \%\left(=1-\mathrm{e}^{-1}\right)$ of the constant voltage charging voltage. Similarly, on discharge, $\mathrm{RC}$ specifies the time it takes the capacitor to discharge to $36.8 \%\left(=\mathrm{e}^{-1}\right)$ of its initial voltage. The RC-time constant also enters into considerations concerning how rapidly energy can be extracted from the capacitor and also how fast power can be provided by the capacitor (refer to discussions relevant to this issue in Appendix B). In Figure A-44 are shown the RC-time constant (in seconds) as a function of the constant-current discharge current for capacitors \#1021, \#1022 and \#1023 at $23^{\circ} \mathrm{C}$ for constant-current discharge tests conducted using a discharge voltage range of $3 \mathrm{~V}$ to $1.5 \mathrm{~V}$. The RC-time constants are seen not to be linear functions of the constant-current discharge current and decrease as the discharge current increases. This is because both the ESR and capacitance values of these capacitors generally decreased with increasing discharge current at this temperature. The $\mathrm{RC}$ values are essentially the same for all three capacitors with device \#1021 having the smallest, and device $\# 1022$ having the largest, $\mathrm{RC}$-time constant over the $10 \mathrm{~A}$ to $100 \mathrm{~A}$ discharge current range with values decreasing from 2.6-3.1 seconds to 0.8-1.0 seconds. In Figure A-45 are shown the $23^{\circ} \mathrm{C}$ RCtime constant values for devices $\# 1021, \# 1022$ and $\# 1023$ as a function of discharge current that resulted from those tests conducted using a $3 \mathrm{~V}$ to $0.01 \mathrm{~V}$ discharge range. The $\mathrm{RC}$ values for devices \#1022 and \#1023 increased slightly, but those of \#1021 decreased considerably as compared with those values determined using the $3 \mathrm{~V}$ to $1.5 \mathrm{~V}$ test range. The major reason for the decrease in the RC values for capacitor \#1021 is due to a large decrease in its measured ESR for the larger voltage range test. At this time we have no physical/chemical explanation for this behavior of device \#1021. In Figure A-46 are shown the RC-time constant values as a function of discharge current for capacitor $\# 1022$ for test temperatures of $-20^{\circ} \mathrm{C}, 23^{\circ} \mathrm{C}$ and $55^{\circ} \mathrm{C}$ for tests that used a $3 \mathrm{~V}$ to $1.5 \mathrm{~V}$ discharge range. In general, the RC-time constant decreased with increasing operating temperature. This general trend is also observed in the data shown in Figure A-47 that displays the RC-time constant as a function of discharge current for capacitor \#1022 at temperatures of $-20^{\circ} \mathrm{C}$ (discharge voltage range of $3 \mathrm{~V}$ to $0.05 \mathrm{~V}$ ) and $23^{\circ} \mathrm{C}$ (discharge voltage range of $3 \mathrm{~V}$ to $0.01 \mathrm{~V}$ ). For a $10 \mathrm{~A}$ constant-current discharge, the $\mathrm{RC}$ values were 3.3 seconds at $23^{\circ} \mathrm{C}$ that increased to 4.7 seconds at $-20^{\circ} \mathrm{C}$. At a $100 \mathrm{~A}$ discharge, $\mathrm{RC}$ was equal to 1 second at $23^{\circ} \mathrm{C}$ and increased to 6.4 seconds at $-20^{\circ} \mathrm{C}$.

All three capacitors were tested at $55^{\circ} \mathrm{C}$ using a voltage discharge range of $3 \mathrm{~V}$ to $1.5 \mathrm{~V}$. From these tests the corresponding RC-time constant could be obtained as a function of discharge current. The time constants are presented in Figure A-48 as a function of discharge current. The $\mathrm{RC}$ values are highly nonlinear functions of the discharge current and, when compared with the values shown in Figure A-44, they do not decrease as rapidly with increasing discharge current. The $\mathrm{RC}$ values at $55^{\circ} \mathrm{C}$ are smaller at the lower discharge currents $(<50 \mathrm{~A})$ than at $23^{\circ} \mathrm{C}$, but are somewhat larger at the $100 \mathrm{~A}$ discharge current level.

\section{Discussion of Ideal-Capacitor Model}

Comparisons to Ideal Capacitor Model. The issue of how well the ideal-capacitor model and the assumption of a constant capacitance for the capacitor will now be examined in further detail. In Figure A-49 are plotted the experimental charge and discharge voltage for capacitor \#1022 during a $10 \mathrm{~A}$ charge and discharge constant-current test (cycle \#2) at $23^{\circ} \mathrm{C}$ as a 
function of charge and discharge time as compared to the time dependent voltage as calculated using the ideal-capacitor model given by equation (3) for charging and equation (4) for discharging. The values for $R_{c}=0.001417 \mathrm{ohms}, C_{c}=2367.5 \mathrm{~F}$ and $V_{o}=0.00099$ volts were used for the charging equation; $R_{c}=0.001408$ ohms, $C_{c}=2373.0 \mathrm{~F}$ and $V_{o}=3.00015 \mathrm{~V}$ were used for the discharge equation. It can be seen in Figure A-49 that the ideal-capacitor model does not fit the data very well except at the voltage limits of $3 \mathrm{~V}$ and $\sim 0.01 \mathrm{~V}$. The measured voltage increased faster than predicted by the ideal-capacitor model during the charging, and decreased slower than predicted during discharging. Figure A-50 more graphically illustrates the voltage difference between the experimental and model predictions. Since the slope of the voltage versus time is proportional to $1 / \mathrm{C}$, this result would imply that the capacitance of the capacitor actually depends on the voltage (charge) on the capacitor and is not a constant.

Further to illustrate this point, we have used equations (7) and (10) to calculate the voltage dependence of the charging capacitance and discharging capacitance respectively. The results are shown in Figure A-51 where the charging and discharging capacitance are shown as a function of the voltage on the capacitor. It should be pointed that the voltage shown on this graph is not the experimental test voltage, but the experimental voltage corrected for the IR factor; i.e., the voltage shown is the capacitor's voltage that is calculated using equation (6) for charging and equation (8) for discharging. From these results it is seen that the capacitor's charging capacitance increases quite rapidly over the $0 \mathrm{~V}$ to $\sim 0.2 \mathrm{~V}$ range, and then continues to increase less rapidly from $\sim 0.2 \mathrm{~V}$ to $3 \mathrm{~V}$. The capacitance reached at $3 \mathrm{~V}$ is very close to that determined using equation (65) which integrates the ampere hour (charge) put into the capacitor over the voltage range divided by the voltage range to determine the capacitance. During the constant-current discharge, the capacitance is seen in Figure A-51 to be very close to that calculated using equation (65) at $3 \mathrm{~V}$, but decreases as the voltage on the capacitor decreases, and at $\sim 0.3 \mathrm{~V}$, decreases quite rapidly to zero. Figures A-52 and A-53 present similar treatments of the experimental voltage as a function of test time, the ideal-capacitor model calculations, and the difference in the experimental-theory voltage as a function of the test voltage for a $100 \mathrm{~A}$ constant-current test (cycle \#2) of capacitor $\# 1022$ at $23^{\circ} \mathrm{C}$. The values used in the idealcapacitor model equations were for charging $R_{c}=0.0003926$ ohms, $C_{c}=2225.4 \mathrm{~F}$ and $V_{o}$ $=0.00107 \mathrm{~V}$, and discharging $R_{d}=0.0004143 \mathrm{ohms}, \mathrm{C}_{d}=2276.1 \mathrm{~F}$ and $\mathrm{V}_{\mathrm{o}}=3.00000 \mathrm{~V}$. As can be seen in these figures, there is a greater disparity between the experimental and model results as compared with the 10 A results. Figure A-54 presents the calculated voltage dependent capacitance for charging and discharging as a function of the capacitor's voltage. It is again observed that both the charge and discharge capacitance is highly dependent on the capacitor voltage. Only at voltage levels approaching $3 \mathrm{~V}$ do the capacitance values approach those measured by the usual method, equation (65).

Figures A-55 to A-60 present the same treatment of the experimental and model results for capacitor \#1022 for charge and discharge tests using $10 \mathrm{~A}$ and $100 \mathrm{~A}$ constant-current tests at $-20^{\circ} \mathrm{C}$. The values used for the ideal-capacitor model were: (a) for charging $R_{c}=0.002541$ ohms, $C_{c}=2337.3 \mathrm{~F}$ and $V_{o}=0.04814 \mathrm{~V}$; and for discharging $R_{d}=0.002068 \mathrm{ohms}$, $\mathrm{C}_{\mathrm{d}}=2300.9 \mathrm{~F}$ and $\mathrm{Vo}=3.00000 \mathrm{~V}$ for the $10 \mathrm{~A}$ tests (Figure A-55); and (b) for charging $\mathrm{R}_{\mathrm{c}}=$ $0.004351 \mathrm{ohms}, \mathrm{C}_{\mathrm{c}}=1898.9 \mathrm{~F}$ and $\mathrm{V}_{\mathrm{o}}=0.00000 \mathrm{~V}$; and for discharging $\mathrm{R}_{\mathrm{d}}=0.003591 \mathrm{ohms}, \mathrm{C}_{d}$ $=1798.9 \mathrm{~F}$ and $\mathrm{V}_{\mathrm{o}}=3.00000 \mathrm{~V}$ for the $100 \mathrm{~A}$ tests (Figure A-58). The differences between the measured voltage and the calculated voltage can be quite large as is shown in Figures A-56 and 
A-59. Figures A-57 and A-60, that display the charging and discharging capacitance as a function of the capacitor's voltage, again show that the capacitance is not a constant, but is different during charging and discharging, and also depends on the capacitor's voltage. Note that in Figure A-60 both the charging and discharging capacitance can be greater than that measured by the usual method at voltages less than the upper voltage limit of $3 \mathrm{~V}$.

When the data acquired from a $250 \mathrm{~A}$ constant-current charge and discharge test is treated in a similar fashion the discrepancy between the ideal-capacitor model and the experimental data is even greater. In Figures A-61 and A-62 are shown the experimental and model calculations for the $250 \mathrm{~A}$ tests at $-20^{\circ} \mathrm{C}$ on capacitor $\# 1022$. As these figures indicate, the discrepancy can be as much as $\pm 0.9 \mathrm{~V}$. It should also be pointed out that the time increment for collection of the charging data was a factor of ten less than that used for the discharge data so that not as many data points appear in the charging data set in these figures. The values used for the idealcapacitor model for this $250 \mathrm{~A}$ test were for charging $R_{c}=0.0008359 \mathrm{ohms}, \mathrm{C}_{\mathrm{c}}=1372.3 \mathrm{~F}$ and $\mathrm{V}_{\mathrm{o}}=0.00000 \mathrm{~V}$; and for discharging $\mathrm{R}_{\mathrm{d}}=0.001593 \mathrm{ohms}, \mathrm{C}_{\mathrm{d}}=1216.7 \mathrm{~F}$ and $\mathrm{V}_{\mathrm{o}}=3.00008 \mathrm{~V}$. Figure A-63 shows the voltage dependence of the capacitance quite dramatically as it illustrates that under extreme conditions of test temperature and high charge/discharge rates the capacitor's capacitance can vary widely from that determined assuming that the capacitance is constant, independent of the capacitor's voltage (or charge).

Constant-current testing was also conducted at $55^{\circ} \mathrm{C}$, as has been discussed previously. The issue of the voltage dependence of the capacitance was also addressed using these test data sets. Unfortunately, the constant-current tests were only conducted for a charging range of $1.5 \mathrm{~V}$ to $3 \mathrm{~V}$, and a discharge range of $3 \mathrm{~V}$ to $1.5 \mathrm{~V}$ (except for one test as will be described below). These tests, however, allowed the comparison of the ideal-capacitor model to the experimental data as was done above. Figures A-64 and A-65 present the ideal-capacitor model results in comparison with the experimental data for a 10 A charge/discharge; Figures A-66 and A-67 show the $100 \mathrm{~A}$ results. The values used in the ideal-capacitor model were: (a) for charging $\mathrm{R}_{\mathrm{c}}=0.0001452 \mathrm{ohms}, \mathrm{C}_{\mathrm{c}}=2660.1 \mathrm{~F}$ and $\mathrm{V}_{\mathrm{o}}=1.51064 \mathrm{~V}$; and for discharging $R_{d}=0.003906$ ohms, $C_{d}=2504.9 \mathrm{~F}$ and $V_{o}=3.00000 \mathrm{~V}$ for the $10 \mathrm{~A}$ tests; and (b) for charging $\mathrm{R}_{\mathrm{c}}=0.0003212 \mathrm{ohms}, \mathrm{C}_{\mathrm{c}}=2356.1 \mathrm{~F}$ and $\mathrm{V}_{\mathrm{o}}=1.59304 \mathrm{~V}$; and for discharging $R_{d}=0.0008121$ ohms, $C_{d}=2363.3 \mathrm{~F}$ and $V_{o}=3.00008 \mathrm{~V}$ for the $100 \mathrm{~A}$ tests. The $10 \mathrm{~A}$ comparison between the model and the experimental data show a rather small difference, particular the discharge data and model predictions. The comparisons for the $100 \mathrm{~A}$ data and the model are not quite as good as the deviations are larger. The calculated voltage dependence of the charge/discharge capacitances at $10 \mathrm{~A}$ are shown in Figure A-68; the $100 \mathrm{~A}$ results are shown in Figure A-69. As discussed in Appendix B, the calculations of the voltage dependence of the charge/discharge capacitances shown in Figures A-68 and A-69 are not really correct since the limited voltage range of $1.5 \mathrm{~V}$ to $3 \mathrm{~V}$ on charge, and $3 \mathrm{~V}$ to $1.5 \mathrm{~V}$ on discharge, do not allow the measurement of the charge (in coulombs or ampere hours) on the capacitor between $\sim 0 \mathrm{~V}$ and $1.5 \mathrm{~V}$ on charge, and between $1.5 \mathrm{~V}$ and $\sim 0 \mathrm{~V}$ on discharge. This term is represented by $Q_{o}$ in equation (7) for $C_{c}$ (i.e., the charging capacitance) and in equation (10) for $C_{d}$ (i.e., the discharging capacitance). Therefore, the voltage dependence of the charge/discharge capacitance show in Figures A-68 and A-69 is correct with regard to how the capacitance changes with the capacitor's voltage, but its absolute magnitude is not. 
Fortunately, one set of constant-current charge/discharge tests at $200 \mathrm{~A}$ was run on capacitor $\# 1022$ at $55^{\circ} \mathrm{C}$ over the charge/discharge voltage range of $\sim 0 \mathrm{~V}$ to $3 \mathrm{~V}$ which can be used for comparison with the other previously described results. As before, the charge and discharge experimental voltages and the ideal-capacitor model voltages as a function of test time are shown in Figure A-70; and the difference between the experimental and model voltages as a function of test voltage are given in Figure A-71. The values used for the ideal-capacitor model were for charging $R_{c}=0.0007652 \mathrm{ohms}, C_{c}=2177.4 \mathrm{~F}$ and $V_{o}=0.04990 \mathrm{~V}$; and for discharging $R_{d}=0.0007196$ ohms, $C_{d}=2221.7 \mathrm{~F}$ and $V_{o}=3.00023 \mathrm{~V}$ for these $200 \mathrm{~A}$ tests. Once again, there are rather large deviations between the experimental data and the model calculations, suggesting that the capacitance is not constant, but is voltage dependent. The voltage dependence of the capacitance was calculated using equations (7) and (10) for charging and discharging respectively. The results are shown in Figure A-72 compared with those constant capacitance values calculated using equation (65). As before, the charge/discharge capacitance is highly dependent on the capacitor's voltage.

An additional way of observing that the capacitance may be voltage dependent is by referring to the definition of capacitance [as discussed in Appendix B, equation (1)] as CV $=\mathrm{Q}$, i.e., capacitance is the constant of proportionality between the voltage on the capacitor, $\mathrm{V}$, and its total stored charge, $Q$. If the capacitance is indeed constant, then a plot of $Q$ versus $V$ should be a straight line with a positive slope equal to the capacitance, $C$, and an intercept equal to zero (i.e., if the capacitor's ESR is equal to zero). To determine if this method of analyzing the experimental data is useful in this regard, in Figure A-73 are plotted the experimental charge stored in capacitor \#1022, as determined by summing the product of the constant-current charging current and the time increment between measurements (i.e., the sum of the I $\Delta t$ product), as a function of the capacitor's voltage as calculated using equation (6) which subtracts the ESRinduced IR voltage jump from the test voltage. These data are for three constant-current charging tests (cycle \#2) on capacitor \#1022 at currents of $10 \mathrm{~A}, 100 \mathrm{~A}$ and $250 \mathrm{~A}$ measured at $20^{\circ} \mathrm{C}$. Upon careful examination (hold a straightedge to the data), the data as plotted in this manner are not straight lines, but are nonlinear, i.e., the slope of the capacitor charge versus capacitor voltage plot changes, thus indicating that the capacitance varies with the voltage (charge) on the capacitor. Similarly, in Figure A-74 are plotted the capacitor's charge as a function of the capacitor's voltage for three constant-current discharge tests (cycle \#2) at $10 \mathrm{~A}$, $100 \mathrm{~A}$ and $250 \mathrm{~A}$ for capacitor $\# 1022$ at $-20^{\circ} \mathrm{C}$. In this plot, the charge on the capacitor was taken as the charge put into the capacitor during the charging cycle minus the charge that was removed during the constant-current discharge as a function of the voltage on the capacitor. The slope of these data plots is again seen to be highly nonlinear, again showing that the capacitance is not constant, but depends on the voltage (charge) on the capacitor as has been previously shown.

\section{Stored and Extracted Energy from Constant-Current Tests}

Since the experimental constant-current discharge data have yielded values for the discharge ESR, $R_{d}$, and the discharge capacitance, $C_{d}$, as functions of the discharge current, $I_{d}$, then from equation (16) in the Appendix the total energy that can be extracted from the capacitor, not including that removed by the voltage drop due to $I_{d} R_{d}$, can be calculated. In Figure A-75 are plotted the Maximum Extractable Energy $=(1 / 2) C_{d}\left(3-I_{d} R_{d}\right)^{2}$, equation (16), where the 3 in the 
expression arises due the fact that the capacitors were charged to $3 \mathrm{~V}$, as a function of the constant-current discharge current for capacitor $\# 1022$ for test temperatures of $-20^{\circ} \mathrm{C}, 23^{\circ} \mathrm{C}$ and $55^{\circ} \mathrm{C}$. As can be seen from these data, the maximum extractable energy is the highest for $55^{\circ} \mathrm{C}$, which decreases at $23^{\circ} \mathrm{C}$, and further decreases at $-20^{\circ} \mathrm{C}$. The $55^{\circ} \mathrm{C}$ energy decreases fairly linearly with increasing discharge current, that at $23^{\circ} \mathrm{C}$ is somewhat nonlinear, and at $-20^{\circ} \mathrm{C}$ the decrease is quite nonlinear. From the data collected during the constant-current tests it was possible to determine the energy extracted during the constant-current discharge tests as a function of the discharge current. This measured energy includes that lost due to the $I_{d}{ }^{2} R_{d}$ losses arising from the ESR of the capacitor as well as the energy extracted during the constant-current discharge test. In Figure A-76 are shown the Measured Energy Extracted from capacitor \#1022 at $-20^{\circ} \mathrm{C}, 23^{\circ} \mathrm{C}$ and $55^{\circ} \mathrm{C}$ as a function of the discharge test current. The energy extracted is given over two discharge voltage ranges for $-20^{\circ} \mathrm{C}(3 \mathrm{~V}$ to $0.05 \mathrm{~V}$, and $3 \mathrm{~V}$ to $1.5 \mathrm{~V}$ that were from the same test but the data were analyzed for the two voltage ranges), over two voltage ranges at $23^{\circ} \mathrm{C}(3 \mathrm{~V}$ to $0.01 \mathrm{~V}$, and $3 \mathrm{~V}$ to $1.5 \mathrm{~V}$ where the data were from two separate tests), and over just one voltage range at $55^{\circ} \mathrm{C}$ (the only constant-current tests conducted at this temperature were over a voltage range of $3 \mathrm{~V}$ to $1.5 \mathrm{~V}$ ). All of the Measured Energy Extracted values decreased with increasing discharge current. They decreased fairly linearly with increasing discharge current for the $23^{\circ} \mathrm{C}$ and $55^{\circ} \mathrm{C}$ temperatures, but decreased nonlinearly at $20^{\circ} \mathrm{C}$. Those energy values measured at $-20^{\circ} \mathrm{C}$ and $23^{\circ} \mathrm{C}$ over the two voltage test ranges followed closely the expected $3 / 4$ decrease in extracted energy when the voltage range was $3 \mathrm{~V}$ to $1.5 \mathrm{~V}$ as compared with when it was $3 \mathrm{~V}$ to $\sim 0 \mathrm{~V}$ [refer to equations (11) and 12)]. These results certainly show that in order for these capacitors to supply the maximum amount of energy they should be operated at the higher temperatures. However, caution presumably would need to be exercised not to exceed the decomposition temperature of the electrolyte.

\section{Constant-Power Test Results}

The constant-power tests conducted on capacitors \#1021, \#1022 and \#1023 will now be discussed. Examples of the constant-power test sequence (three of these test cycles were used in the tests at each constant-power discharge) are shown in Figures A-77 and A-78. The test voltage as a function of test time is shown in Figure A-77 (Maccor tester) for a test on capacitor $\# 1022$ at $23^{\circ} \mathrm{C}$ that included a $50 \mathrm{~A}$ constant-current charge from $1.5 \mathrm{~V}$ to $3 \mathrm{~V}$, followed by a voltage clamp at $3 \mathrm{~V}$ for 10 seconds, and then a $100 \mathrm{~W}$ constant-power discharge from $3 \mathrm{~V}$ to $1.5 \mathrm{~V}$, and, finally, a voltage clamp for 10 seconds at $1.5 \mathrm{~V}$. For those tests that used a Bitrode tester a 20-second voltage clamp was used instead of the 10 seconds that was used with the Maccor tester. The IR-jump due to the ESR of the capacitor is evident at the beginning of the charge and the IR-drop due to the ESR of the capacitor can be seen at the beginning of the constant-power discharge. Figure A-78 shows the test current as a function of test time corresponding to the test voltage shown in Figure A-77. Shown in Figure A-79 is the discharge voltage as a function of discharge time for the constant-power tests at $10 \mathrm{~W}$ to $100 \mathrm{~W}$ for capacitor \#1021 (Maccor tester was used). Figure A-80 displays the results for tests ranging from $100 \mathrm{~W}$ to $330 \mathrm{~W}$ (Bitrode tester was used). For these tests, a $10 \mathrm{~A}$ constant-current charge was used for the $10 \mathrm{~W}, 20 \mathrm{~W}, 100 \mathrm{~W}, 200 \mathrm{~W}, 250 \mathrm{~W}$ and $330 \mathrm{~W}$ tests; a $50 \mathrm{~A}$ constant-current charge was used for the $50 \mathrm{~W}$ and $100 \mathrm{~W}$ tests. In a similar manner, the discharge voltage as a function of discharge time for the constant-power tests for capacitor \#1022 are shown in Figures A-81 and A-82, and for capacitor \#1023 in Figures A-83 and A-84. From these data, and using 
equation (74), the specific energy as a function of the specific power was determined. The results at $23^{\circ} \mathrm{C}$ are presented in Figure A-85 as a linear-linear plot and in Figure A-86 as a log$\log$ plot for the three capacitors. There is some scatter in the data, especially between those values measured using the Maccor tester $(10 \mathrm{~W}$ to $100 \mathrm{~W}$ ) and the Bitrode tester (100 W to 330 $\mathrm{W}$ ). The specific energy for the three capacitors varied between approximately $3.85 \mathrm{~W} \cdot \mathrm{h} / \mathrm{kg}$ to $3.98 \mathrm{~W} \cdot \mathrm{h} / \mathrm{kg}$ at approximately $15.7 \mathrm{~W} / \mathrm{kg}$, decreasing in a fairly linear manner to approximately $3.5 \mathrm{~W} \cdot \mathrm{h} / \mathrm{kg}$ to $3.6 \mathrm{~W} \cdot \mathrm{h} / \mathrm{kg}$ at approximately $530 \mathrm{~W} / \mathrm{kg}$. The specific energy volumetric density as a function of the specific power volumetric density were also determined for the three capacitors at $23^{\circ} \mathrm{C}$. The values are plotted in Figure A-87. These values were calculated using equation (75) with the volume of the capacitor calculated from the dimensions supplied by Maxwell Energy Products, Inc. (i.e., 0.462 liters). The specific energy volumetric density varied from $5.24 \mathrm{~W} \cdot \mathrm{h} / \mathrm{L}$ to $5.5 \mathrm{~W} \cdot \mathrm{h} / \mathrm{L}$ at $21.7 \mathrm{~W} / \mathrm{L}$, decreasing to $4.73 \mathrm{~W} \cdot \mathrm{h} / \mathrm{L}$ to $4.95 \mathrm{~W} \cdot \mathrm{h} / \mathrm{L}$ at $714.8 \mathrm{~W} / \mathrm{L}$. Capacitor \#1023 displayed the best energy and power performance of the three devices. The specific energy and specific power of these devices are well below those set by the near-term (1997) goals set by PNGV of power/energy $>100$, specific energy $=6 \mathrm{~W} \cdot \mathrm{h} / \mathrm{kg}$ and energy density $=9 \mathrm{~W} \cdot \mathrm{h} / \mathrm{L}$, as well as the long-term (2006) PNGV goals of power/energy $>100$, specific energy $=18 \mathrm{~W} \cdot \mathrm{h} / \mathrm{kg}$ and energy density $=24 \mathrm{~W} \cdot \mathrm{h} / \mathrm{L}[1,2]$.

Constant-power tests were also conducted at temperatures of $55^{\circ} \mathrm{C}$ on capacitors $\# 1021$, $\# 1022$ and $\# 1023$ besides the $23^{\circ} \mathrm{C}$ tests described above. The discharge voltage as a function of discharge time for a series of constant-power tests at $10 \mathrm{~W}, 20 \mathrm{~W}, 50 \mathrm{~W}, 100 \mathrm{~W}, 200 \mathrm{~W}, 250 \mathrm{~W}$ and $330 \mathrm{~W}$ (a constant-current of $10 \mathrm{~A}$ was used to charge the capacitor) at a temperature of $55^{\circ} \mathrm{C}$ for capacitor \#1022 are shown in Figures A-88 and A-89 over the discharge voltage range of $3 \mathrm{~V}$ to $1.5 \mathrm{~V}$. These tests were all done using a Maccor tester on a test channel that had higher current and power capabilities than that used for the previously discussed $23^{\circ} \mathrm{C}$ tests. The same sequence of constant-power tests $(10 \mathrm{~W}, 20 \mathrm{~W}, 50 \mathrm{~W}, 100 \mathrm{~W}, 200 \mathrm{~W}, 250 \mathrm{~W}$ and $330 \mathrm{~W})$ at a test temperature of $-20^{\circ} \mathrm{C}$ over the discharge voltage range of $3 \mathrm{~V}$ to $1.5 \mathrm{~V}$ were also conducted on capacitor \#1022. As before, a $10 \mathrm{~A}$ constant-current charge was used on all of these tests. The discharge voltage as a function of discharge time for the various constant-power discharge tests are shown in Figures A-90 and A-91. Upon close comparison of the data in Figures A-88 and A-89 with those in Figures A-90 and A-91 the discharge voltage can be observed to decrease more rapidly with time at $-20^{\circ} \mathrm{C}$ as compared with the $55^{\circ} \mathrm{C}$ tests (and the $23^{\circ} \mathrm{C}$ tests that are shown in Figures A-81 and A-82). This more rapid voltage decrease results from the capacitor having a smaller capacitance and, therefore, a smaller stored energy at $-20^{\circ} \mathrm{C}$ than at the higher temperatures. The specific energy as a function of specific power was calculated from the $55^{\circ} \mathrm{C}$ and $-20^{\circ} \mathrm{C}$ data using equation (74). The results are plotted in Figure 92 that compares the specific energy as a function of specific power for all of the measurements on the three capacitors at all three test temperatures. The highest specific energy was observed to occur at $23^{\circ} \mathrm{C}$. The specific energy was lower at $55^{\circ} \mathrm{C}$ and it also decreased more rapidly with increasing specific power than was observed at $23^{\circ} \mathrm{C}$. The values at $55^{\circ} \mathrm{C}$ for the three capacitors ranged from $3.6 \mathrm{~W} \cdot \mathrm{h} / \mathrm{kg}$ to $3.8 \mathrm{~W} \cdot \mathrm{h} / \mathrm{kg}$ at $15.7 \mathrm{~W} / \mathrm{kg}$, decreasing in a fairly linear manner to $3.0 \mathrm{~W} \cdot \mathrm{h} / \mathrm{kg}$ to 3.4 $\mathrm{W} \cdot \mathrm{h} / \mathrm{kg}$ at $520 \mathrm{~W} / \mathrm{kg}$. At $-20^{\circ} \mathrm{C}$ the specific energy (capacitor \#1022) was reduced even further. The specific energy at $-20^{\circ} \mathrm{C}$ decreased quite rapidly with increasing specific power compared with those at the higher test temperatures. The value of the specific energy was $3.6 \mathrm{~W} \cdot \mathrm{h} / \mathrm{kg}$ at a specific power of $15.8 \mathrm{~W} / \mathrm{kg}$, decreasing linearly to $0.98 \mathrm{~W} \cdot \mathrm{h} / \mathrm{kg}$ at $521 \mathrm{~W} / \mathrm{kg}$. 
As discussed in Appendix B, by knowing the discharge ESR, $R_{d}$, of the capacitor as a function of the constant-current discharge (and temperature), it is possible to calculate the maximum power that can be provide by the capacitor during a constant-current discharge. Using equation (22), $P_{d \text { max }}=V_{o}^{2} /\left(4 R_{d}\right)$, and a working voltage of $V_{o}=3 V$ the value of $P_{d, \text { max }}$ as a function of the constant-current discharge current were calculated for capacitor $\# 1022$ at $-20^{\circ} \mathrm{C}$, $23^{\circ} \mathrm{C}$ and $55^{\circ} \mathrm{C}$. The results are presented in Figure A-93. The highest available power would be at $55^{\circ} \mathrm{C}$ for discharge currents $<100 \mathrm{~A}$, at which point the power available at $23^{\circ} \mathrm{C}$ would be higher. The higher power available at $100 \mathrm{~A}$ is due to the much lower discharge ESR at $23^{\circ} \mathrm{C}$ compared with that measured at $55^{\circ} \mathrm{C}$. Unfortunately, we were unable to measure the discharge ESR at $23^{\circ} \mathrm{C}$ for discharge currents greater than $100 \mathrm{~A}$ to determine if this trend in the $\mathrm{P}_{\mathrm{d} \text {,max }}$ values would continue at the higher discharge currents. The available power at $-20^{\circ} \mathrm{C}$ would be considerably lower than at the higher temperatures due to the larger discharge ESR values measured at this temperature. The observed nonlinearity in the $P_{d, \max }$ data is due to the observed nonlinearity in the discharge ESR of the capacitor as a function of discharge current (refer to Figure A-41). As is pointed out in Appendix B, $P_{d \max }$, as calculated using equation (22) is for an efficiency of only $1 / 3$ or $\sim 33.3 \%$. The maximum discharge energy that corresponds to $P_{d, \max }$ can also be calculated using equation (24) as is given in Appendix $B$, i.e., $E_{d, \max }=C_{d} V_{0}^{2} / 8$ where $C_{d}$ is the measured discharge capacitance that is a function of the constant-current discharge current and $V_{o}$ is the working voltage, which in the present case is $3 \mathrm{~V}$. The calculated values for $E_{d, \max }$ as a function of the discharge current for capacitor $\# 1022$ at the three test temperatures of $-20^{\circ} \mathrm{C}$, $23^{\circ} \mathrm{C}$ and $55^{\circ} \mathrm{C}$ are given in Figure A-94. The greatest energy would be at $55^{\circ} \mathrm{C}$ that decreases as the temperature is lowered and also as the discharge current is increased. The decrease with increasing discharge current is fairly linear at all three temperatures. Equations (22) and (24) used in the above calculations were derived assuming a constant value of the discharge ESR that is independent of the constant-current discharge current. Therefore, the calculated values for $P_{d, \max }$ and $E_{d, \max }$ shown in Figures A-93 and A-94 would be those values if the ESR of the capacitor was constant and equal to the $R_{d}$ values used. The fact that the ESR may vary with temperature does not influence the derivation of the equations used, but simply reflects the fact that $P_{d, \text { max }}$ and $E_{d, \text { max }}$ may be temperature dependent.

\section{Constant-Current and Constant-Power Discharge Efficiency}

The constant-current and constant-power test data can be used to calculate the efficiencies for the constant-current and constant-power discharge tests for the capacitors at the three different test temperatures. Using equation (72) given in Appendix B for the round-trip efficiency of a constant-current charge/discharge cycle, the efficiency as a function of the charge/discharge current was calculated from the constant-current test data for capacitor \#1022 at temperatures of $-20^{\circ} \mathrm{C}, 23^{\circ} \mathrm{C}$ and $55^{\circ} \mathrm{C}$. The results are given in Figure A-95. The highest measured efficiency of $\sim 1$ was observed at a charge/discharge current of $100 \mathrm{~A}$ at $55^{\circ} \mathrm{C}$. The $55^{\circ} \mathrm{C}$ efficiencies were observed to increase with increasing charge/discharge current, reaching a maximum at $100 \mathrm{~A}$, and then decreased with further increases in the charge/discharge current. Over the charge/discharge current test range of $10 \mathrm{~A}$ to $100 \mathrm{~A}$, the efficiencies at $23^{\circ} \mathrm{C}$ decreased with increasing charge/discharge current, decreasing from $\sim 94 \%$ at $10 \mathrm{~A}$ to $\sim 90 \%$ at $100 \mathrm{~A}$. The $-20^{\circ} \mathrm{C}$ efficiencies were lower than those at the higher temperatures and decreased very rapidly with increasing charge/discharge current. At 10 A the efficiency was $~ 92 \%$, decreasing to $\sim 32 \%$ at $250 \mathrm{~A}$. 
As also discussed in Appendix B, the round-trip constant-power discharge efficiencies can be determined from the constant-power tests. The relevant equation to use here is equation (76) given in Appendix B. In this instance, a constant-current charge of $10 \mathrm{~A}$ was used in the present tests and a series of constant-power discharges were then studied as has been previously described. Analyzing the constant-power tests using equation (76) for capacitor \#1022 at the three test temperatures, the efficiencies were determined and are shown in Figure A-96 as a function of the constant-power discharge power. Here, the highest efficiencies were measured for those tests conducted at $23^{\circ} \mathrm{C}$ where the efficiency was $\sim 98 \%$ at $10 \mathrm{~W}$, increasing to a maximum of $\sim 99 \%$ at $50 \mathrm{~W}$, and then decreasing to $\sim 96 \%$ at $100 \mathrm{~W}$. At $55^{\circ} \mathrm{C}$, the efficiency was a maximum at $20 \mathrm{~W}(\sim 96 \%)$, and decreased to an efficiency of $\sim 85 \%$ at $330 \mathrm{~W}$. The efficiency at $-20^{\circ} \mathrm{C}$ was a maximum at $20 \mathrm{~W}(\sim 96 \%)$ that decreased quite rapidly to $\sim 25 \%$ at $330 \mathrm{~W}$.

Further considerations of the efficiencies associated with the constant-current discharge of a capacitor are considered in Appendix B, where relationships between the energy that can be provided by a capacitor at a given efficiency and the energy stored in it [equations (35) and (36)] are derived and discussed. The power that can be provided by a capacitor at a given efficiency as a function of the energy stored in it [equations (37) and (38)] are also given in Appendix B. Both the energy and power are also dependent on the voltage to which the capacitor is charged. Assuming that for practical applications an efficiency of $90 \%$ is desired, by using equation (37) and the experimental values for the constant-current discharge current dependence of the discharge ESR, the corresponding maximum power that can be produced by capacitor \#1022 at temperatures of $-20^{\circ} \mathrm{C}, 23^{\circ} \mathrm{C}$ and $55^{\circ} \mathrm{C}$ were determined. In Figure $\mathrm{A}-97$ are shown the results of these calculations. Actually, the same values for the power as were shown in Figure A-93 are plotted here but they are reduced by a factor of $4\left[2 n(1-n) /(1+n)^{2}\right]=\sim 0.1995$ for an efficiency of $90 \%$, i.e., $n=0.9$ [refer to equation (44)]. The highest power available at an efficiency of $90 \%$ from capacitor \#1022 at constant-current discharges $<100 \mathrm{~A}$ would be provided if the capacitor was operated at $55^{\circ} \mathrm{C}$. At a discharge current of $100 \mathrm{~A}$, the highest power at $90 \%$ efficiency would occur if the capacitor was at $23^{\circ} \mathrm{C}$. Because no discharge data was acquired at $23^{\circ} \mathrm{C}$ at discharge currents greater than $100 \mathrm{~A}$ from which the discharge ESR could be determined, it is not known if the dramatic increase in the power at $90 \%$ efficiency as seen in Figure A-97 at a $100 \mathrm{~A}$ discharge would continue for higher discharge currents. The lowest discharge power at $90 \%$ efficiency would be if the capacitor was at $-20^{\circ} \mathrm{C}$, but the trend of the data is for an increasing power at discharge currents greater than $250 \mathrm{~A}$. The corresponding energy that could be provided by capacitor $\# 1022$ at the three test temperatures at $90 \%$ efficiency as a function of the constant-current discharge current was calculated using equation (35) as given in Appendix B. Shown in Figure A-98 are the results of this calculation. Actually the same values of the energy that were plotted in Figure A-94, i.e., $E_{d, \max }$ at $P_{d, \max }$, are plotted in Figure A-98 except they have been multiplied by a factor of $4\left[2 n /(1+n)^{2}\right]=3.59$ for an efficiency of $90 \%$, i.e., $n=0.9$ [refer to equations (24) and (36) in Appendix B]. The maximum energy that would be available from capacitor \#1022 would be if it were operated at $55^{\circ} \mathrm{C}$. At a $10 \mathrm{~A}$ constant-current discharge, the energy is $\sim 2.82 \mathrm{~W} \cdot \mathrm{h}$, decreasing to $\sim 2.53 \mathrm{~W} \cdot \mathrm{h}$ at $200 \mathrm{~A}$. A lower amount of energy would be available at $23^{\circ} \mathrm{C}$ where at $10 \mathrm{~A}$ the energy is $-2.67 \mathrm{~W} \cdot \mathrm{h}$, decreasing to $\sim 2.56$ $\mathrm{W} \cdot \mathrm{h}$ at $100 \mathrm{~A}$. At $-20^{\circ} \mathrm{C}$, the available energy decreases even further where at $10 \mathrm{~A}$ the energy at $90 \%$ efficiency is $\sim 2.58 \mathrm{~W} \cdot \mathrm{h}$ that decreases to $\sim 1.34 \mathrm{~W} \cdot \mathrm{h}$ at a discharge current of $250 \mathrm{~A}$. At all three test temperatures, the amount of available energy at $90 \%$ efficiency decreased in a 
somewhat linear manner as the constant-current discharge current increased as can be observed in Figure A-98.

\section{Leakage-Current Test Results}

Leakage-current tests, which measure the current required to maintain, in this case, a voltage of $3 \mathrm{~V}$ on the capacitor, were conducted on the three capacitors only at $23^{\circ} \mathrm{C}$. The collected test data are shown in Figure 99. Capacitor \#1021 was not very good as its leakage current stabilized at approximately 85 milliamperes after $\sim 8$ hours, while devices $\# 1022$ and \#1023 have leakage currents of approximately 10 milliamperes after $\sim 21$ hours. The corresponding equivalent parallel resistance (EPR) values can be calculated from these data using equation (77) in Appendix $B$ with $V_{0}=3 \mathrm{~V}$. The results for the equivalent parallel resistance as a function of test time, $R_{p}(t)$, are shown in Figure A-100. Devices \#1022 and \#1023 were essentially the same having an EPR value of $\sim 300$ ohms after 24 hours on test while that of \#1021 was $~ 3.5$ ohms after 8 hours (the total test time on this capacitor). Compared with the previously discussed values of the ESR that were in the milliohm range, it is a good approximation to neglect the EPR when discussing the ideal-capacitor model as applied to constant-current and constant-power tests.

\section{Self-Discharge Test Results}

The self-discharge test, which measures the voltage decrease of the capacitor as a function of time when it is not connected to a load, was conducted on all three capacitors at $23^{\circ} \mathrm{C}$ and $55^{\circ} \mathrm{C}$, and on capacitor $\# 1022$ at $-20^{\circ} \mathrm{C}$. Shown in Figure A-101 is the measured capacitor voltage for the three capacitors at a test temperature of $23^{\circ} \mathrm{C}$ as a function of test time after each capacitor had been charged to $3 \mathrm{~V}$ using a $10 \mathrm{~A}$ constant-current charge, and then held at $3 \mathrm{~V}$ for 30 minutes before the start of the self-discharge test. Capacitor \#1021 exhibited much worse performance than devices \#1022 and \#1023 as its voltage decreased to $\sim 1.4 \mathrm{~V}$ after 48 hours while the voltage of capacitor \#1022 decreased to $\sim 2.5 \mathrm{~V}$, and the voltage of capacitor \#1023 decreased to $\sim 2.65 \mathrm{~V}$.

The self-discharge test not only provides information concerning the rate at which the voltage on a capacitor declines with time in the open-circuit state and, thereby, the amount of energy left stored in the capacitor, but the test data can also be analyzed to determine what physical and/or chemical mechanisms may be responsible for the observed voltage decrease. Electrochemical capacitors in the charged condition, like batteries, are in a state of high energy relative to that of the system in the discharged state. There is, therefore, a driving force corresponding to the free energy of discharge that tends to diminish the charge if some mechanism(s) of self-discharge exist by which that discharge can take place [11-14]. Selfdischarge of a charged capacitor can only occur if some Faradaic electron-transfer process(es) can take place at and below the maximum potential. An ideal-capacitor has no self-discharge or current-leakage pathway and, hence, can remain charged indefinitely. Practical capacitors, however, like batteries, can suffer self-discharge over a period of time so that this phenomenon is of major interest in the evaluation of capacitor (and battery) performance and does influence the materials and fabrication methods used in their production. Conway [11,12] has identified three potential causes of self-discharge. Case (1) can arise if the self-discharge occurs from an ohmic 
leakage between the pair of double-layer electrodes constituting one cell of the capacitor. The self-discharge in this case is simply that of a decline in voltage represented by a parallel-RC circuit so that the time dependent behavior of the capacitor's voltage would be of the form $\ln \left[\mathrm{V}(\mathrm{t}) / \mathrm{V}_{\mathrm{o}}\right]=-\mathrm{t} / \mathrm{RC}$ or, $\mathrm{V}(\mathrm{t})=\mathrm{V}_{\mathrm{o}} \exp (-\mathrm{t} / \mathrm{RC})$. Thus, $\mathrm{V}(\mathrm{t})$ declines exponentially with time $[6,7]$. In this expression, $V_{0}$ is the initial voltage on the capacitor, $R$ is the value of the parallel resistance, and $\mathrm{C}$ is the capacitance of the capacitor. Case (2) is when the capacitor electrode material and/or its electrolyte contain impurities that are oxidizable or reducible over the potential range corresponding to the potential difference attained in the capacitor. If these impurities are present in small concentrations, then the self-discharge redox process(es) is (are) diffusion-controlled. This (these) process(es) generally lead $[6,7]$ to a functional form for the decrease in the capacitor potential having the form $V_{o}-V(t) \propto t^{1 / 2}$, i.e., the voltage as a function of time should decrease approximately as the square-root of the test time. Case (3) can result from overcharging the capacitor beyond the respective " + " or "-" decomposition potential of the electrolyte. This leakage process corresponds to a Faradaic, potential-dependent charge-transfer reaction. An equivalent circuit representation of the behavior of the electrode interphase or double-layer would be a charge leakage process that corresponds to a potential-dependent Faradaic resistance operating in parallel across the double-layer capacitance; its value increasing with declining potential. This case results in a functional form of the self-discharge voltage represented by $V_{0}-V(t) \propto \ln (t+$ const. $)$, i.e., the voltage of the capacitor declines logarithmically with time [6,7]. In order to acquire a feeling for how these models of the capacitor self-discharge process(es) account for the observed data, the experimental self-discharge voltage data for the capacitors have been fit to the three functional forms that represent each of the three cases. In Figure A-101 the fit to an exponential function of time, Case (1), is shown for the three capacitors at a test temperature of $23^{\circ} \mathrm{C}$. In these fits the initial voltage, $\mathrm{V}_{0}$, was held at $3 \mathrm{~V}$ so that at time equal to zero the exponential fit was forced to be equal to three volts. The values for the best regression analysis fit is shown as well as the square of the correlation coefficient $\left(\mathrm{R}^{2}\right)$ of the fit to the data. Obviously the fit is not very good for any of the capacitor data sets. The three data sets were also analyzed using the Case (2) model. The results of the regression analysis fits to a square-root dependence on the time is given in Figure A-102. Here, the fits are perhaps a little better, but do not fit the entire self-discharge voltage range of the data. The three test data sets were then plotted for the Case (3) process, i.e., a logarithmic time dependence, and are shown in Figure A-103. The data plotted in this form is obviously not a straight line over the entire voltage range, but, perhaps, there may be limited voltage and time ranges where the correlation may be applicable. A more extensive analysis of the data that uses various voltage/time ranges fit to the three cases may yield more definitive correlations, but this has not been done in the present study.

The self-discharge data can also be used to determine the Self-Discharge Energy Loss Factor (SDLF), which, as discussed in Appendix B, is a measure of how much energy has been lost from the capacitor as a function of time due to the self-discharge process. Using equation (78) given in Appendix B, the time dependence of the SDLF $(t)$ was calculated for each of the three capacitors from the test data given in Figure A-101. The results are presented in Figure A-104 for capacitors \#1021, \#1022 and \#1023 for a temperature of $23^{\circ} \mathrm{C}$. The best capacitor was \#1023 that lost $\sim 22 \%$, after 48 hours, of the initial stored energy it had at $3 \mathrm{~V}$. The next best device was \#1022 that lost $~ 32 \%$ of its initial stored energy after 48 hours, followed by 
capacitor \#1023 that lost $\sim 78 \%$ of its stored energy after this same period.

The self-discharge test was also done on all three of the capacitors at $55^{\circ} \mathrm{C}$. The voltage data is plotted as a function of time in Figure A-105. In this figure, and in Figures A-106 to A-108, the data are plotted and fit to the Case (1), Case(2) and Case (3) model predictions as was previously described. In this instance, as was the case for the $23^{\circ} \mathrm{C}$ self-discharge data, none of the model cases fit the data except, possibly, for limited voltage/time regions. Figure A-109 presents the calculations for the Self-Discharge Energy Loss Factor as a function of the test time at the $55^{\circ} \mathrm{C}$ test temperature. At this test temperature, capacitor $\# 1023$ again displayed the least amount of energy loss, $\sim 33 \%$ after 48 hours, higher than the $22 \%$ loss is had at $23^{\circ} \mathrm{C}$. Capacitor $\# 1022$ lost $\sim 35 \%$ of its initial energy at $55^{\circ} \mathrm{C}$ compared with the $\sim 32 \%$ it lost at $23^{\circ} \mathrm{C}$. Capacitor \#1021 was again the worst of the three capacitors at this temperature, losing $\sim 72 \%$ of its stored energy after 48 hours. Its performance, however was better at $55^{\circ} \mathrm{C}$ than at $23^{\circ} \mathrm{C}$, where it lost $\sim 78 \%$ of its stored energy after the 48 -hour test period.

The self-discharge test was also done on capacitor $\# 1022$ at $-20^{\circ} \mathrm{C}$; the time dependence of the voltage as a function of test time is shown in Figure A-110. Also shown in this figure, and in Figures A-111 and A-112, are the various linear regression fits to the voltage/time data in accordance with the Case (1), (2) and (3) physical/chemical models for the self-discharge process(es). Once again the model fits to the data over the full self-discharge time range are not good, but there is a time region of $\sim 2$ hours to 48 hours for the Case (3) model (Figure A-112) where the voltage as a function of the $\log _{10}$ of the test time is quite linear. Figure $A-113$ shows the SDLF $(t)$ as a function of test time as calculated from the voltage/time data using equation (78). As can be seen in this figure, after 48 hours capacitor \#1022 lost $\sim 16 \%$ of its stored energy at $-20^{\circ} \mathrm{C}$ compared with its having lost $\sim 32 \%$ at $23^{\circ} \mathrm{C}$ and $\sim 35 \%$ at $55^{\circ} \mathrm{C}$. This is quite an improvement compared with the two higher test temperatures.

In order too more readily visualize the effects of temperature on the self-discharge voltage as a function of test time, in Figures A-114 to A-117 the self-discharge data acquired from capacitor $\# 1022$ at $-20^{\circ} \mathrm{C}, 23^{\circ} \mathrm{C}$ and $55^{\circ} \mathrm{C}$ have been re-plotted on the same graph. The data presented in these figures have been plotted to the Case (1) through Case (3) model predictions and the linear regression fits to the data are also shown as before. Shown in Figure A-114 are the best fits to the Case (1) model in the form an exponential time dependence of $V(t)$ for the entire test time range of the self-discharge data at each of the three test temperatures. The best regression analysis fits are shown by the dashed lines that represent the displayed expressions, an exponential fit to the test time. A value for the quality of fit is given by the $\mathrm{R}^{2}$ value. The regression fits in this figure were forced to have an intercept, i.e., when $t=0$, of $3 \mathrm{~V}$. In Figure A-115, the regression fits were for the case where the intercept value was not fixed, but was treated as an adjustable parameter. The shapes of the trend lines shown in Figures A-114 and A-115 do not really correspond to the entire range of the data curves at any of the test temperatures. Perhaps if this form of the time dependence was applied only over limited ranges of the voltage/time data then better fits, with multiple time constants, could be obtained. The important point to be by the data presented in Figures A-114 and A-115 is that the capacitor's voltage decreased more rapidly as the temperature was increased, i.e., the voltage remained higher longer at the $-20^{\circ} \mathrm{C}$ temperature than at $23^{\circ} \mathrm{C}$ and $55^{\circ} \mathrm{C}$. At $55^{\circ} \mathrm{C}$, the rate of the voltage decrease was initially slower than that observed at $23^{\circ} \mathrm{C}$, but was more rapid after approximately 
19 hours. When the capacitor was tested at $-20^{\circ} \mathrm{C}$, the rate of the voltage decrease is much slower over the entire test period (48 hours). Figure A-116 plots the self-discharge data in a manner corresponding to the Case (2) model that predicts a $\sim \mathrm{t}^{1 / 3}$ time dependence for the capacitor self-discharge voltage. Again the regression fits to the entire self-discharge voltage/time data sets are not very good; but some correlation may exist at the longer test times. Similarly, in Figure A-117 the Case (3) model is applied to the data in the form of a logarithmic time dependence of the self-discharge voltage data. There perhaps may be certain time regions of the voltage versus time data, in particular at the longer test times, over which a linear fit could be obtained, but certainly the major portions of the data do not correspond to this model. Given the rather poor fits to the data, it is not appropriate at this time to discuss in any detail possible temperature dependent physical/chemical processes and their affects when discussing these results. Perhaps as more capacitor self-discharge data are acquired and analyzed by these and additional model predictions concerning the time (and temperature) dependence of the selfdischarge voltage, then a better understanding of this important phenomenon will be obtained.

The self-discharge energy loss factor, $\operatorname{SDLF}(\mathrm{t})$, that was previously presented in Figures A-104, A-109 and A-113 for capacitor \#1022 at the three test temperatures, was re-plotted in Figure A-118 too more readily visualize the effects of temperature on this factor. As can be seen in this figure, the SDLF factor is much lower at $-20^{\circ} \mathrm{C}$ than it is at the two higher test temperatures. Discussed in Appendix B is the fact that the self-discharge energy loss factor can be used to determine how much energy the capacitor loses as a function of time after it has been charged to its working voltage and then left in an open-circuit state. The total potential energy stored in a capacitor is equal to $E(t)=1 / 2 C V(t)^{2}$ where $E$ is the energy in joules stored in the capacitor as a function of time, $C$ is the capacitance of the capacitor in farads, and V(t) is the voltage on the capacitor at a time $t$ after it has been charged to its initial voltage and left in an open-circuit condition. From this relation, a decrease in the voltage on the capacitor will affect its total stored energy as the energy will change as the square of the voltage. The voltage measured as a function of time by the self-discharge test is $\mathrm{V}(\mathrm{t})$ and, as is shown in Appendix B, equation (84), leads to an expression for the energy remaining in the capacitor as a function of time after the self-discharge of the capacitor has begun. If one assumes that the capacitance does not depend on the voltage on the capacitor or on the discharge current, assumptions shown previously not to be the case for capacitor \#1022, then the energy remaining in the capacitor can be calculated using the time dependent self-discharge voltage in the relation $E_{\text {remaining }}=1 / 2 \mathrm{CV}(\mathrm{t})^{2}$, equation (84). Using the capacitance values measured for capacitor \#1022 from the $10 \mathrm{~A}$ constant-current discharge tests at the three temperature, i.e., $\mathrm{C}=2302.7 \mathrm{~F}$ at $-20^{\circ} \mathrm{C}, \mathrm{C}=$ $2373.3 \mathrm{~F}$ at $23^{\circ} \mathrm{C}$, and $\mathrm{C}=2503.9 \mathrm{~F}$ at $55^{\circ} \mathrm{C}$, the energy remaining in the capacitor as a function of time during self-discharge was calculated. The results are shown in Figure A-119 where the energy remaining, in watt-hours, as a function of self-discharge time, in hours, is given for the three temperatures. Initially, at time $t=0$, the capacitor had the greatest amount of stored energy at $55^{\circ} \mathrm{C}(3.13 \mathrm{~W} \cdot \mathrm{h})$ due to its having the highest capacitance at this temperature. The amount of stored energy at time $\mathrm{t}=0$ decreased as the temperature was lowered, $2.97 \mathrm{~W} \cdot \mathrm{h}$ at $23^{\circ} \mathrm{C}$, and $2.87 \mathrm{~W} \cdot \mathrm{h}$ at $-20^{\circ} \mathrm{C}$. However, as the self-discharge test time increased, at $\sim 6$ hours the energy remaining in the capacitor at $-20^{\circ} \mathrm{C}$ was approximately the same as that stored when the capacitor was at $55^{\circ} \mathrm{C}$. However, for times greater than $\sim 6$ hours the energy stored in the capacitor was always greater at $-20^{\circ} \mathrm{C}$ than it was at either $23^{\circ} \mathrm{C}$ or $55^{\circ} \mathrm{C}$. After 48 hours, the energy remaining in the capacitor was $\sim 2.43 \mathrm{~W} \cdot \mathrm{h}$ at $-20^{\circ} \mathrm{C},-2.00 \mathrm{~W} \cdot \mathrm{h}$ at $23^{\circ} \mathrm{C}$, and $\sim 2.06 \mathrm{~W} \cdot \mathrm{h}$ 
at $55^{\circ} \mathrm{C}$. These values correspond to a decrease in the stored energy of $\sim 15 \%$ at $-20^{\circ} \mathrm{C}, \sim 33 \%$ at $23^{\circ} \mathrm{C}$, and $\sim 34 \%$ at $55^{\circ} \mathrm{C}$, not inappreciable amounts after only 48 hours under open-circuit conditions. Consideration of the self-discharge energy loss could be critical for those energy storage applications of capacitors where some provision to periodically recharge the capacitor is not allowed.

As a final examination of the self-discharge test data, in Figure A-120 are compared the self-discharge voltage as a function of discharge time at a test temperature of $23^{\circ} \mathrm{C}$ for capacitor $\# 1142$ from capacitor Set \#1 and capacitor \#1023 from capacitor Set \#2. Each of these capacitors exhibited the best performance in their respective capacitor sets during the self-discharge test, i.e., they each had the smallest amount of voltage decrease as a function of test time. In Figures A-120 through A-122 are re-plotted the self-discharge voltage as a function of selfdischarge time for these two capacitors in order too more readily compare the two devices. In each figure, the best fits to the three model cases again are shown for ease of comparison. Capacitor \#1142 exhibited a more rapid falloff of its voltage than capacitor \#1023. Capacitor \#1142 had a considerable lower capacitance, $157 \mathrm{~F}$, compared with $\sim 2464 \mathrm{~F}$ for capacitor \#1023, but this does not necessarily mean that its voltage should decline more rapidly. The model fits to the three self-discharge cases are also not seen to fit the self-discharge voltage for capacitor \#1142 as has been previously pointed out to be the case for capacitor \#1023.

Comparing the amount of energy lost to self-discharge, capacitor \#1142 lost $\sim 38 \%$ of its initial stored energy at $3 \mathrm{~V}$ (refer to Figure A-18), while capacitor \#1023 lost $\sim 22 \%$ of its initial stored energy at $3 \mathrm{~V}$, also after 48 hours (refer to Figure A-104). Based on the percent of the initial amount of energy lost as a function of time under open-circuit conditions, capacitor \#1023 is better than \#1142. As we do not know the details of the differences in the electrode materials, electrolytes and fabrication techniques used in these two sets of capacitors, we cannot at this time attribute this better performance to any particular cause or physical/chemical process(es).

\section{Set \#3 $^{[8,9]}$}

The $24 \mathrm{~V}, 8$-cell unpackaged capacitors attained specific energy levels of $5 \mathrm{~W} \cdot \mathrm{h} / \mathrm{kg}$ at specific powers as high as $1 \mathrm{~kW} / \mathrm{kg}$. The packaged $24 \mathrm{~V}$ devices suffered some performance loss and fell slightly below the unpackaged device levels achieving specific energy levels of approximately $4.5 \mathrm{~W} \cdot \mathrm{h} / \mathrm{kg}$ at a specific power of $500 \mathrm{~W} / \mathrm{kg}$. The equivalent series resistance of these capacitors, determined from the constant-current tests, when using an organic electrolyte, was measured to be approximately 0.4 to $0.6 \mathrm{ohm}\left(1\right.$ to $\left.1.5 \mathrm{ohm}-\mathrm{cm}^{2}\right)$ at room temperature $\left(25^{\circ} \mathrm{C}\right)$. This value did not change appreciably when tests were performed at $60^{\circ} \mathrm{C}$. As Figure A-123 shows, when the temperature is lowered to $-20^{\circ} \mathrm{C}$, the resistance increased substantially to approximately 1.5 to $2.0 \mathrm{ohm}\left(3.75\right.$ to $\left.5 \mathrm{ohm}-\mathrm{cm}^{2}\right)$. This result is expected for these types of electrolytes. The constant-power discharge behavior (shown in Figure A-124) displays a significant loss of available energy when the temperature was lowered to $-24^{\circ} \mathrm{C}$. Selfdischarge data is presented in Figure A-125 and shows a significant increase in the self-discharge at higher temperatures $\left(60^{\circ} \mathrm{C}\right)$ which again is expected. Round-trip efficiency for these capacitors, as measured by the constant-current charge and discharge tests, was approximately $85 \%$.

Life-cycle tests were also conducted on these capacitors. The data is shown in 
Figure A-126 for a 1 A constant-current discharge and in Figure A-127 for a 1 A constant-current charge. The capacity fell below $80 \%$ of the initial capacity after 110,000 constant-current test cycles. A constant-current test current of $1 \mathrm{~A}$ was used so as to achieve a discharge time of about 18 to 20 seconds. The distinct changes in the watt:hour data as shown in Figures A-126 and A-127 at about 55,000 and 65,000 cycles were due to changes in the test procedure. It should be noted that the specified test procedure was not followed between test cycles 1 to 65,000 in that the voltage clamps at the end of the charge and discharge portions of the cycle were not included until those test cycles after 65,000 . The test data suggests that the addition of the voltage clamps may have had a significant effect on the life-cycle performance of the capacitor as the capacity (watt-hour) of the capacitor was observed to begin to decrease after these changes were made to the test procedure. The apparent increase in the stored energy when the voltage clamp was included in the test sequence arises as the additional charge supplied by the tester during the clamp was erroneously included in the total watthour value. The intermittent "scatter" in the watthour values appears to be an effect of the test equipment, the origin of which is not currently known. 


\section{SUMMARY}

Three different sets of capacitors, based on an experimental design that used carbon electrodes with a non-aqueous electrolyte, were received from Maxwell Energy Products, Inc. for testing by the INEEL Automotive Systems and Technology Department. Set \#1 consisted of three capacitors (capacitor numbers \#1141, \#1142 and \#1143) with nominal ratings of $155 \mathrm{~F}$ to $165 \mathrm{~F}$ at $3 \mathrm{~V}$. Set \#2 consisted of three capacitors (capacitor numbers \#1021, \#1022 and \#1023) that had a nominal rating of $2300 \mathrm{~F}$ at $3 \mathrm{~V}$. Set \#3 consisted of several capacitors with ratings of $2 \mathrm{~F}$ at $24 \mathrm{~V}$.

The capacitors of Set \#1 were tested using the constant-current test, the constant-power test, the leakage-current test and the self-discharge test. All tests were conducted at $23^{\circ} \mathrm{C}$. From the constant-current tests, the equivalent series resistances (ESR) and the capacitances as a function of the constant-current discharge current were determined for all three of the capacitors. The constant-power tests provided information on the specific energy and specific power of the three capacitors. The time dependence of the equivalent parallel resistance of the three devices was determined using the leakage-current test. Using the self-discharge test, the time dependence of the self-discharge energy loss factor was measured for all three capacitors.

The capacitors of Set $\# 2$ were tested to determine the temperature $\left(-20^{\circ} \mathrm{C}, 23^{\circ} \mathrm{C}\right.$ and $55^{\circ} \mathrm{C}$ ) and constant-current discharge current dependence of the ESR and capacitance using the constant-current test. The efficiency of the capacitors during the constant-current tests was also calculated as a function of the temperature and discharge current. The constant-power test was used to measure the specific energy and specific power of the capacitors at three different temperatures $\left(-20^{\circ} \mathrm{C}, 23^{\circ} \mathrm{C}\right.$ and $\left.55^{\circ} \mathrm{C}\right)$. The efficiency of the capacitors for the constant-power test conditions was also determined. The equivalent parallel resistance was measured at $23^{\circ} \mathrm{C}$ as a function of time using the leakage-current test. The self-discharge test was used to measure the time dependence and the effect of temperature on the self-discharge energy loss factor for the three capacitors. The collected data from the tests were also used to calculate the voltage dependence of the capacitance, to discuss various consideration concerning the use of the capacitors to supply energy and power, to calculate the RC-time constants of the devices, and to discuss the self-discharge test data in terms of physical/chemical models that have been proposed to describe this phenomenon.

The Set \# 3 capacitors were tested using constant-current, constant-power, self-discharge and life-cycle tests. The ESR values at $-20^{\circ} \mathrm{C}, 25^{\circ} \mathrm{C}$ and $60^{\circ} \mathrm{C}$ were determined from the constant-current tests. Specific energy and specific power values were measured. The selfdischarge tests were conducted at $-23^{\circ} \mathrm{C}, 23^{\circ} \mathrm{C}$ and $60^{\circ} \mathrm{C}$. Life-cycle tests were conducted to 110,000 constant-current cycles.

The specific energy and specific power of all three sets of capacitors were well below those set by the near-term (1997) goals set by PNGV [1,2] of power/energy $>100$, specific energy $=6 \mathrm{~W} \cdot \mathrm{h} / \mathrm{kg}$ and energy density $=9 \mathrm{~W} \cdot \mathrm{h} / \mathrm{L}$, as well as the long-term (2006) PNGV goals of power/energy $>100$, specific energy $=18 \mathrm{~W} \cdot \mathrm{h} / \mathrm{kg}$ and energy density $=24 \mathrm{~W} \cdot \mathrm{h} / \mathrm{L}$. 


\section{REFERENCES}

1. R. A. Sutula, "The Department of Energy's High-Power Energy Storage Programs," in Preprints of the Annual Automotive Technology Development Customers' Coordination Meeting, Volume I Oral Presentations, October 28-November 1, 1996.

2. A. F. Burke, J. E. Hardin, and E. J. Dowgiallo, "Applications of Ultracapacitors in Electric Vehicle Propulsion Systems," Proceedings of the 34th Power Sources Symposium, Cherry Hill, NJ (1990).

3. J. R. Miller and A. F. Burke, Electric Vehicle Capacitor Test Procedures Manual, Revision 0, DOE/ID-10491 (October 1994).

4. C. J. Farahmandi, J. Dispennette, and E. Blank, "High Power 2,300 Farad Ultracapacitors Based on Aluminum/Carbon Electrode Technology," Proceedings of the Symposium on Electrochemical Capacitors, F. M. Delnick and M. Tomkiewicz, editors, Proceedings Volume 95-29 (The Electrochemical Society, Inc., Pennington, NJ, 1996), p. 187.

5. T. C. Murphy, R. B. Wright, and R. A. Sutula, "U. S. Department of Energy Electrochemical Capacitor Development and Testing Activities,", Proceedings of the Symposium on Electrochemical Capacitors II, F. M. Delnick, D. Ingersoll, X. Andrieu and K. Naoi, eds., Volume 96-25 (The Electrochemical Society, Inc., Pennington, NJ) p. 258.

6. R. B. Wright, T. C. Murphy, S. A. Rogers and R. A. Sutula, "Experimental Electrochemical Capacitor Test Results," to be published in the Proceedings of Symposium Y: Materials for Electrochemical Energy Storage and Conversion II Batteries, Capacitors and Fuel Cells, Materials Research Society Fall Meeting 1997.

7. R. B. Wright, T. C. Murphy, W. E. Kramer and R. A. Sutula, "Recent DOE Experimental Electrochemical Capacitor Test Results,", Proceedings of the Electric Vehicle Symposium Fourteen (EVS-14), December 1997.

8. T. C Murphy and W. E. Kramer, "Electrochemical Capacitor Development for Electric and Hybrid Vehicles," Proceeding of the 37th Power Sources Conference, June 17-20, 1996, p. 140.

9. T. C. Murphy and P. B. Davis, "Electrochemical Capacitors," Proceeding of the Symposium on Electrochemical Capacitors, F. M. Delnick and M. Tomkiewicz, eds., Volume 95-29 (The Electrochemical Society, Inc., Pennington, NJ, 1996) p. 269.

10. J. R. Miller, "Optimization of Electrochemical Capacitors for High-Power Applications," Proceedings of the 4th International Seminal on Double Layer Capacitors and Similar Energy Storage Devices, December 12-14, 1994, Volume 4. 
11. B. E. Conway and J. R. Miller, "Fundamentals and Applications of Electrochemical Capacitors," manual for an Electrochemical Society Short Course, May 4, 1997.

12. B. E. Conway, T-C Liu, and W. G. Pell, "Experimental Evaluation and Interpretation of Self-Discharge and Recovery Behavior of $\mathrm{RuO}_{2}$ and Carbon Electrodes," Proceedings of the 6th International Seminal on Double Layer Capacitors and Similar Energy Storage Devices, December 9-11, 1996, Volume 6.

13. P. Kurzweil and O. Schmid, "High Performance Metal Oxide Supercapacitors," Proceedings of the 6th International Seminal on Double Layer Capacitors and Similar Energy Storage Devices, December 9-11, 1996, Volume 6.

14. S. L. Roberson, D. Finello, R. F. Davis, T. Liu, W. Pell and B. E. Conway, "Behavior of Molybdenum Nitrides for Electrochemical Capacitors," Proceedings of the 6th International Seminal on Double Layer Capacitors and Similar Energy Storage Devices, December 9-11, 1996, Volume 6. 


\begin{abstract}
APPENDIX A
GRAPHIC FIGURES

FOR

MAXWELL ENERGY PRODUCTS, INC. CAPACITORS

SET \#1, SET \#2 AND SET \#3
\end{abstract}


A-2 


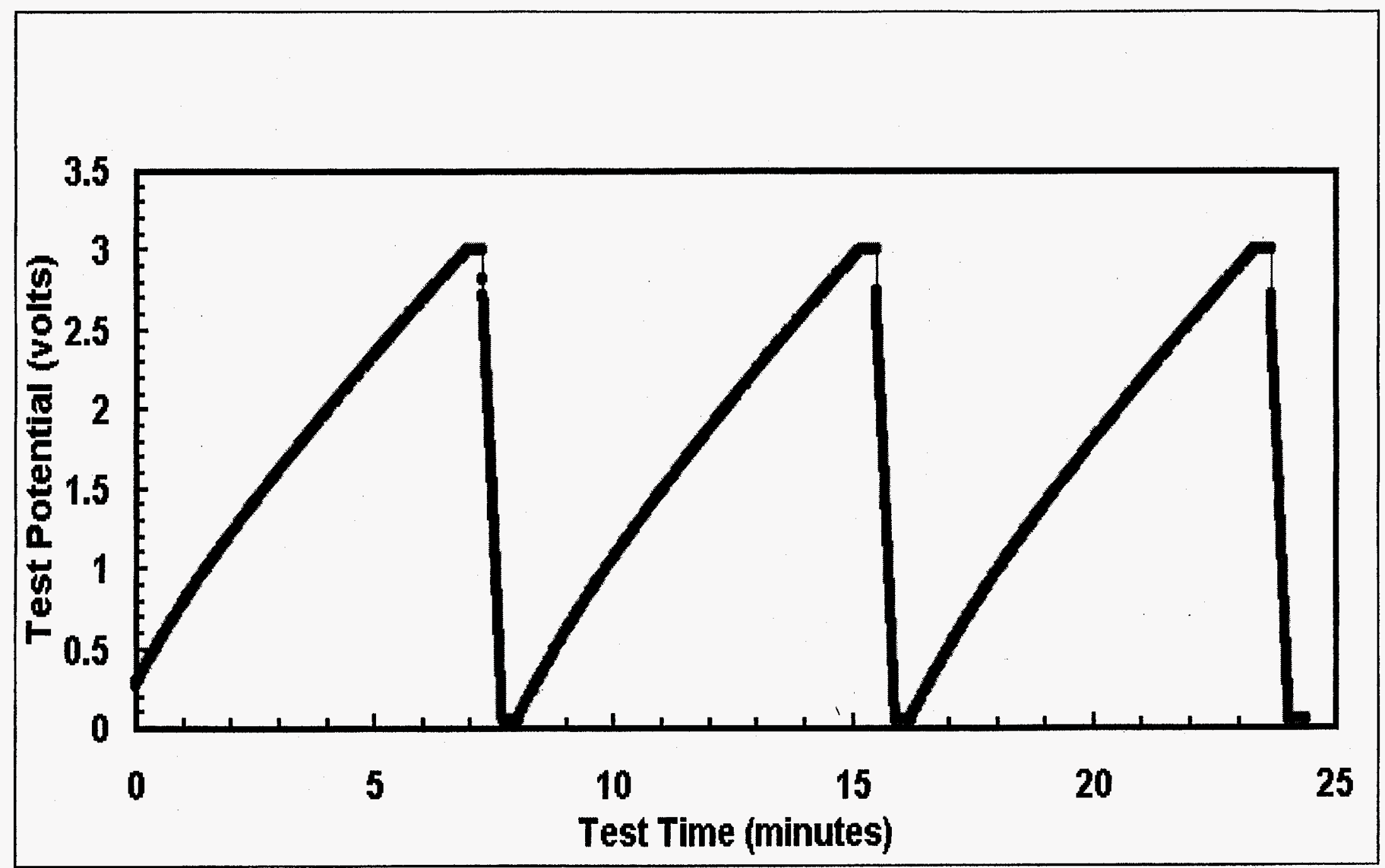

Figure A-1. Data for capacitor \#1143 from constant-current test cycle \#2 at 1 A charge and 16 A discharge. 


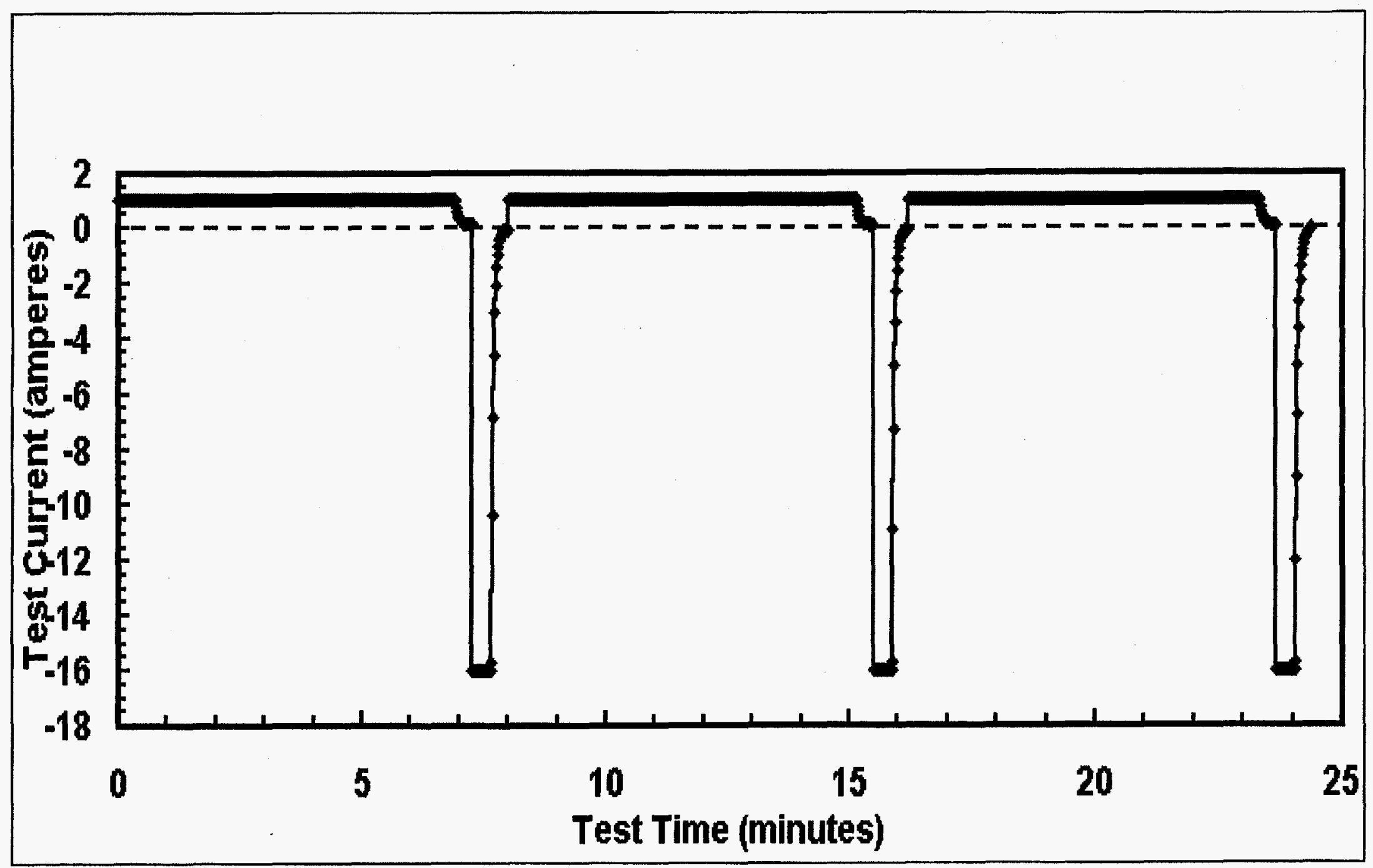

Figure A-2. Data from constant-current test cycle \#2 for capacitor \#1143 at $1 \mathrm{~A}$ charge and $16 \mathrm{~A}$ discharge. 


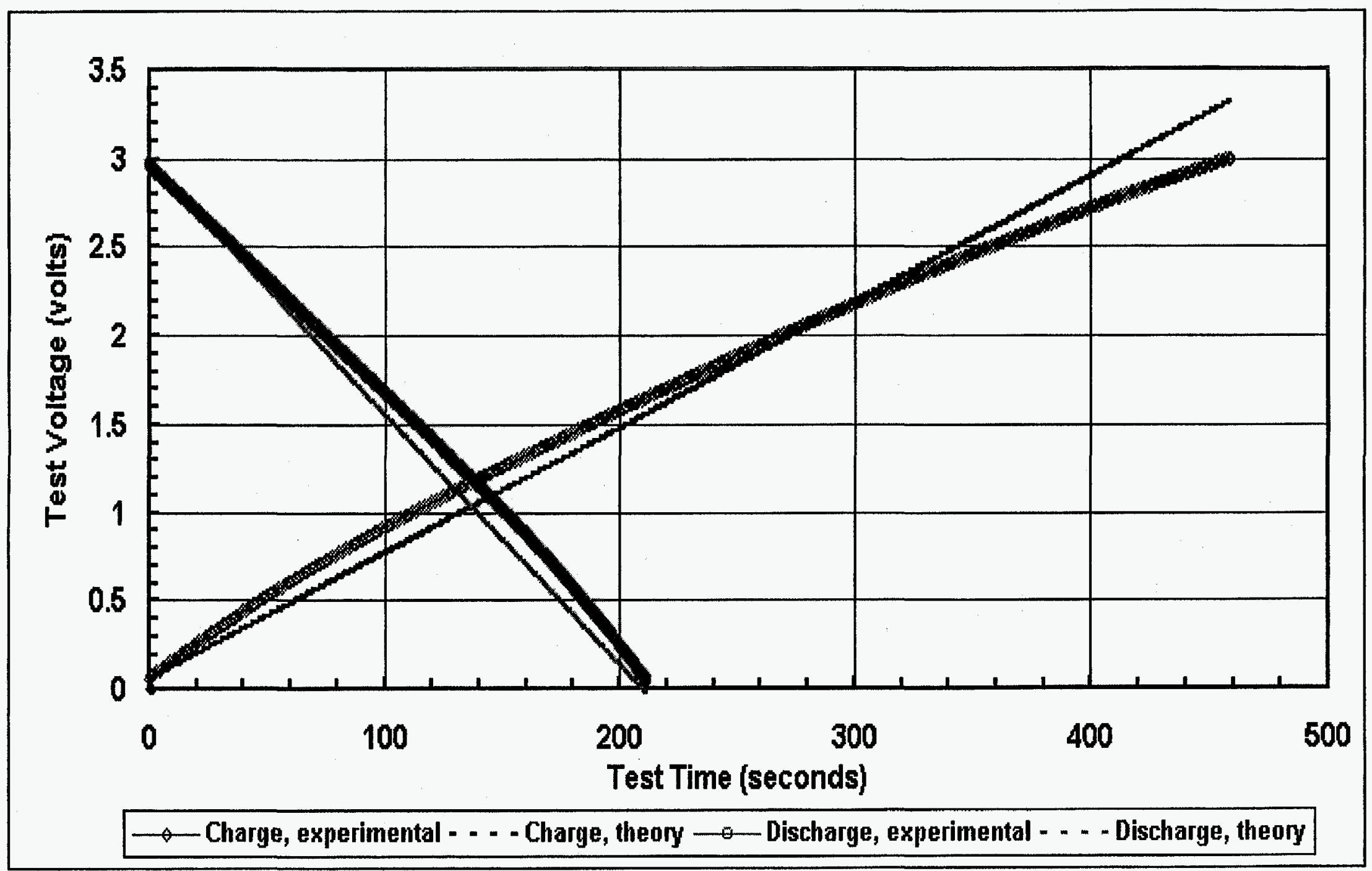

Figure A-3 (a). Data for capacitor \#1143 for a 1 A constant-current charge and a 2 A constant-current discharge. 


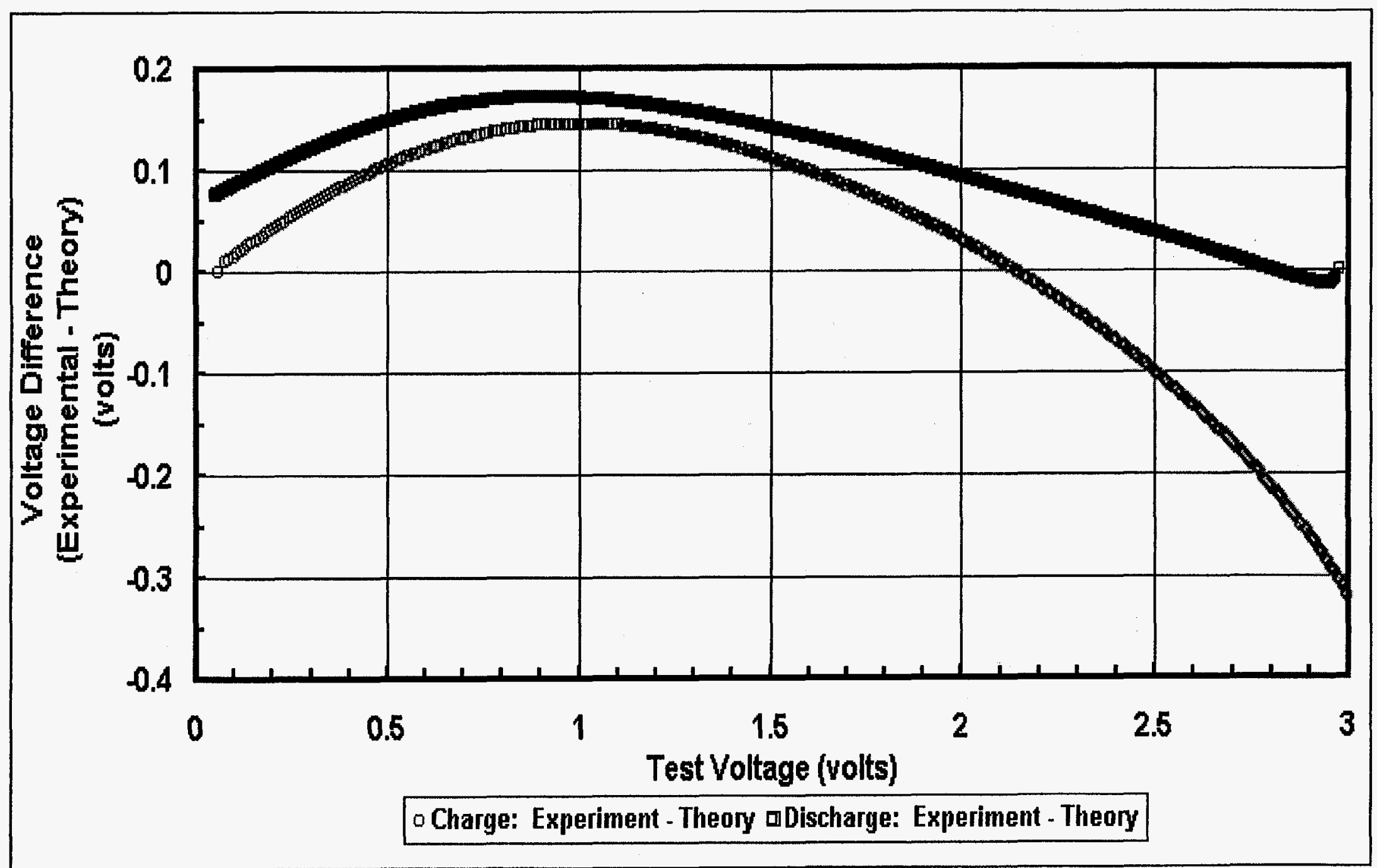

Figure A-3 (b). Potential difference (Experimental - Theory) for capacitor \#1143 for a 1 A charge and a 2 A discharge test cycle. 


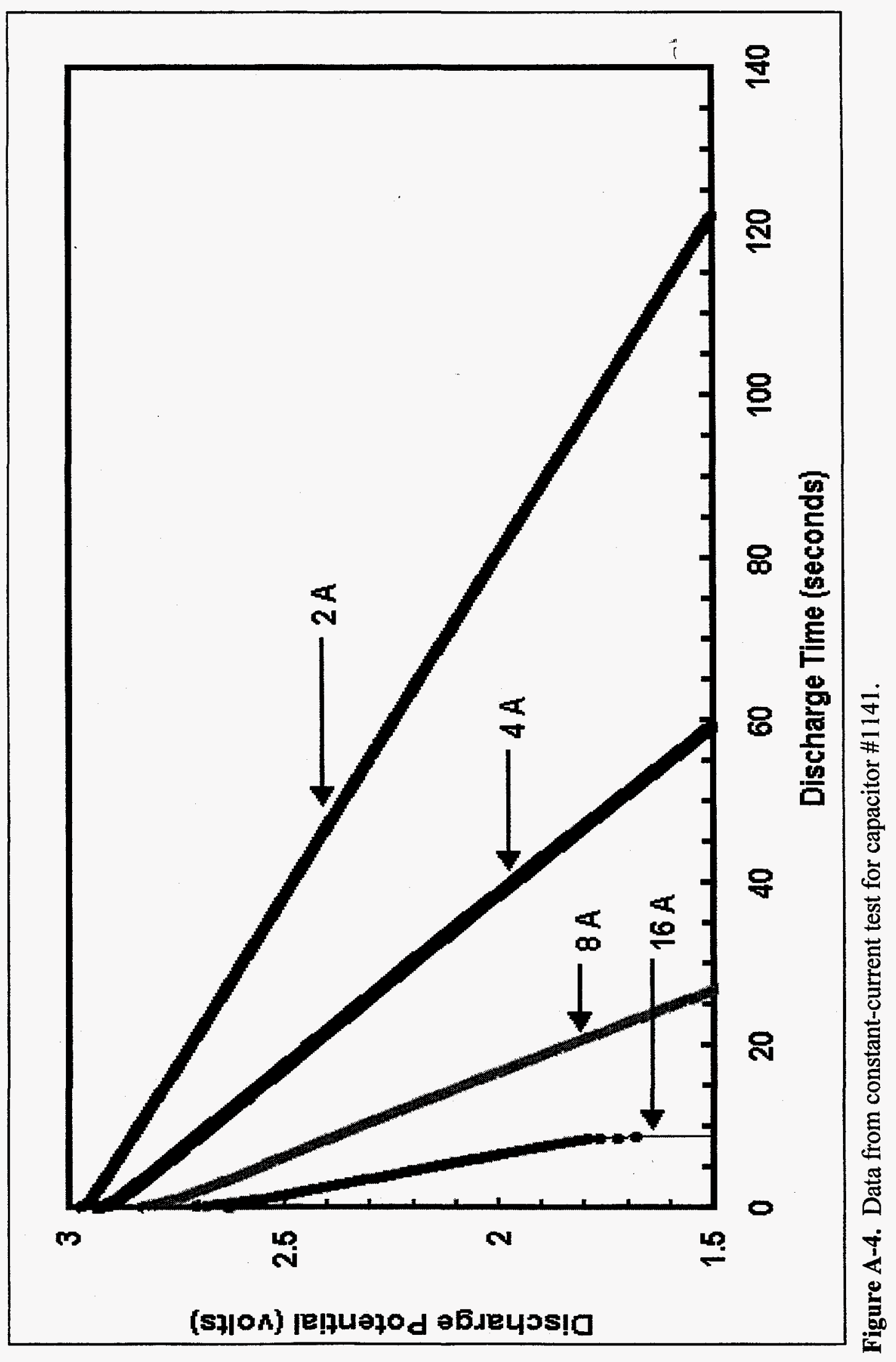




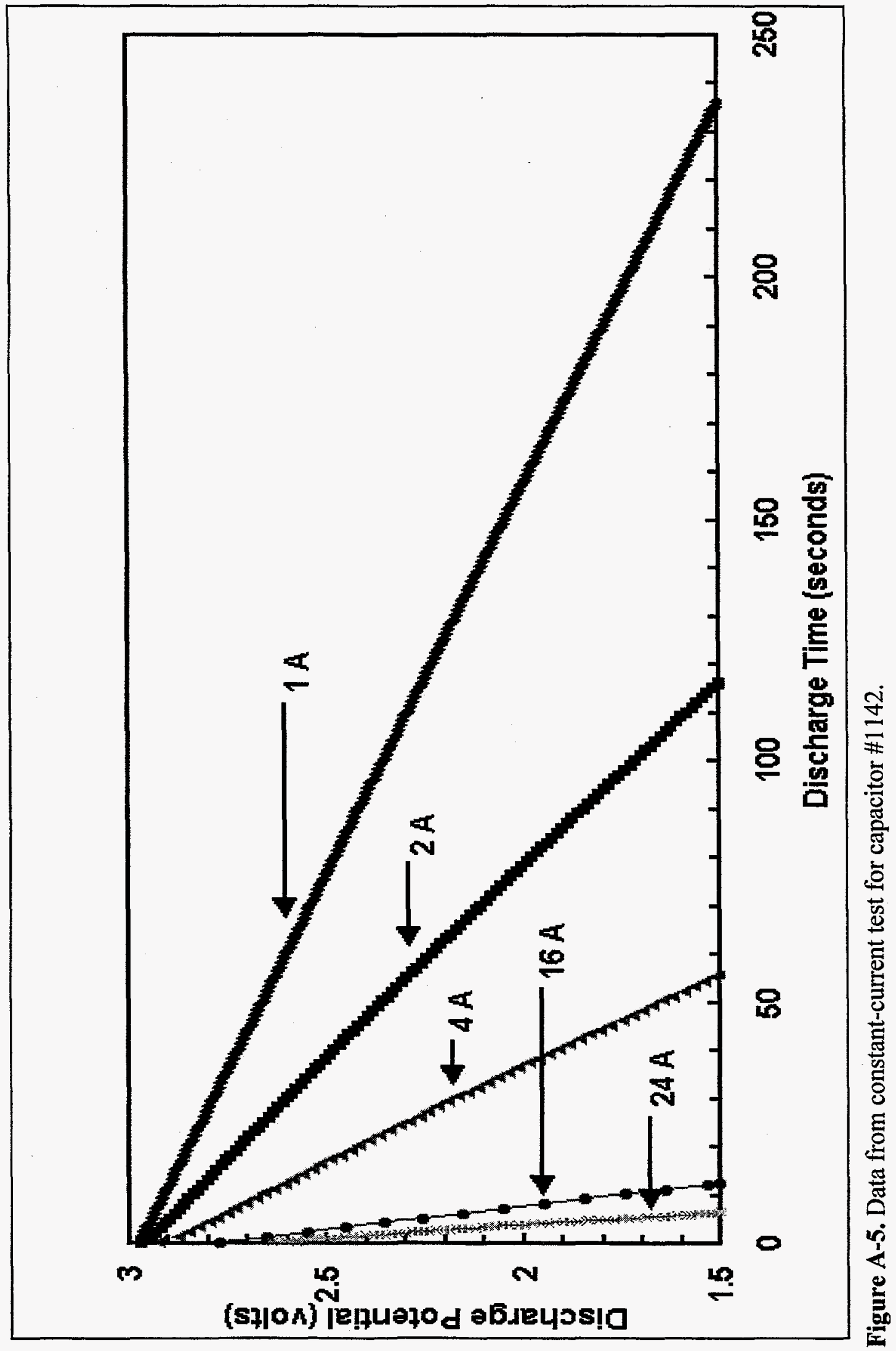




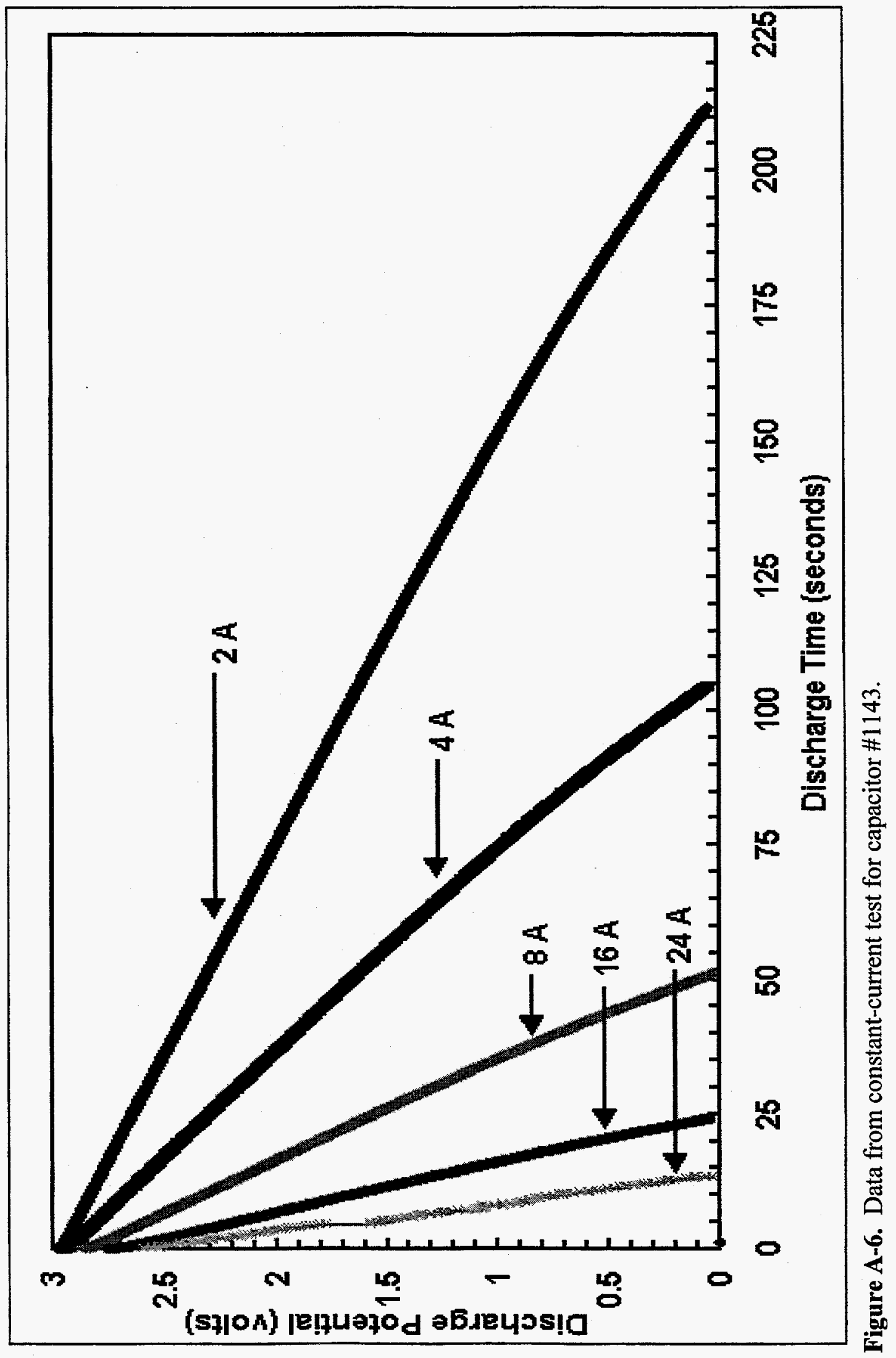




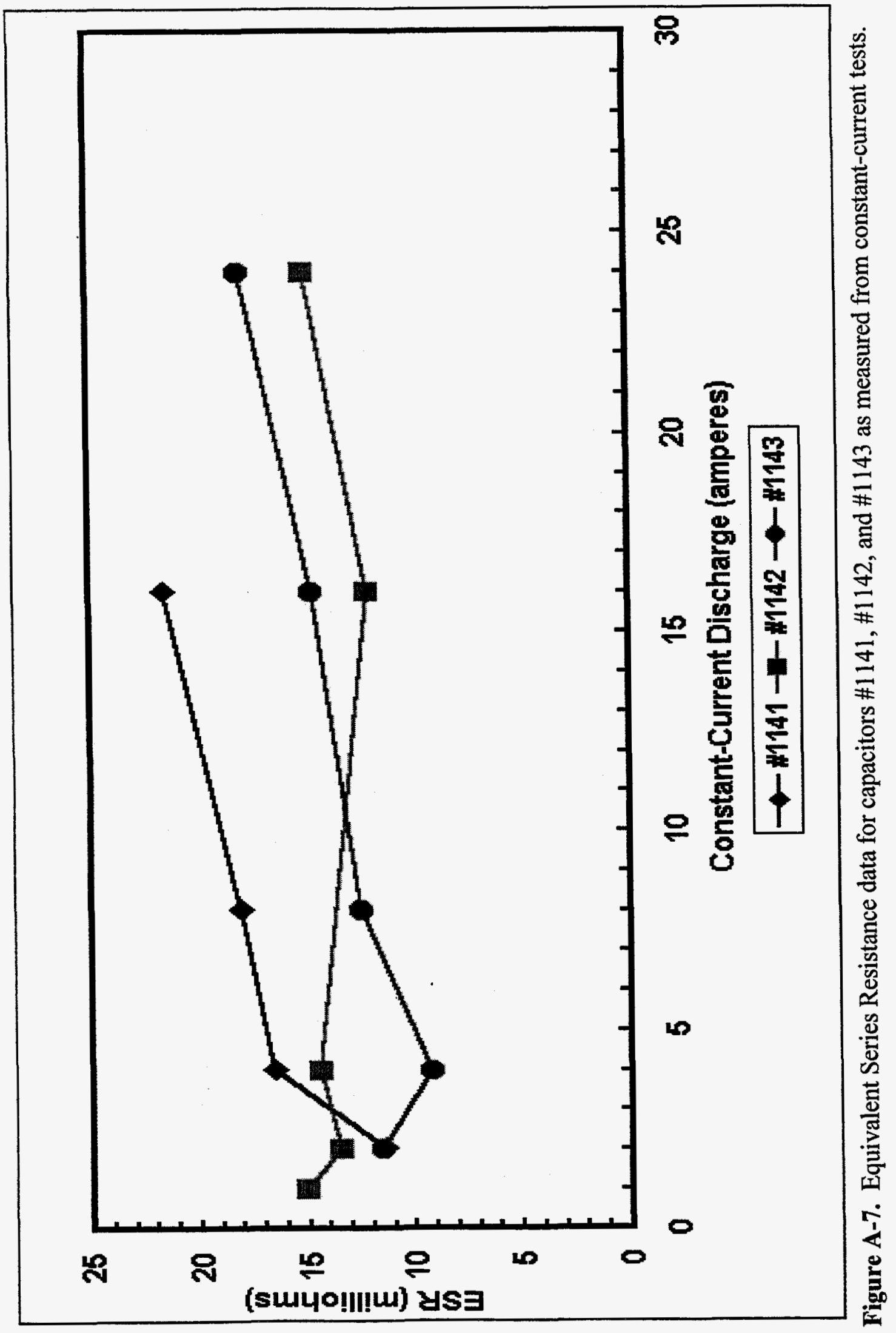




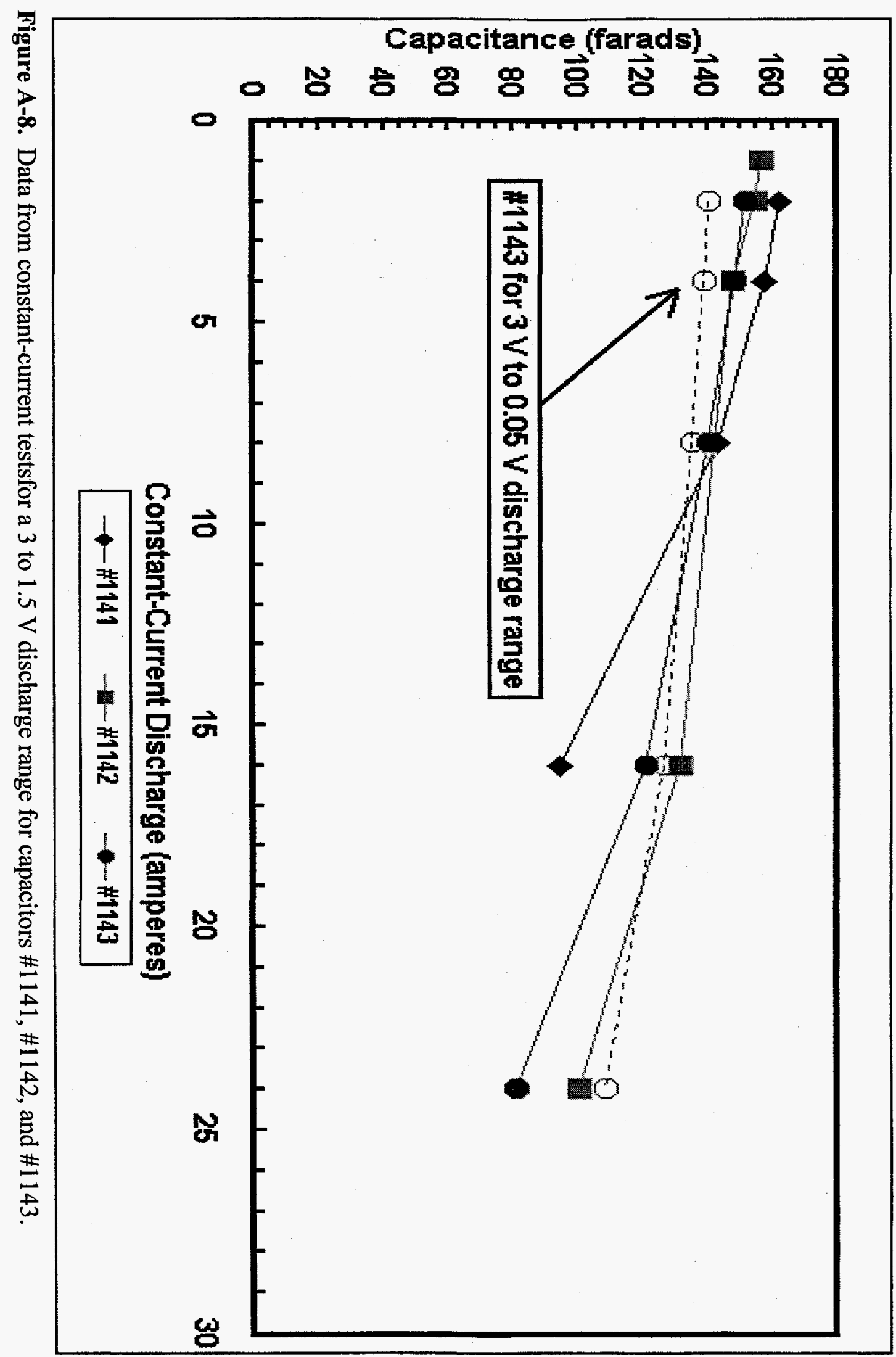




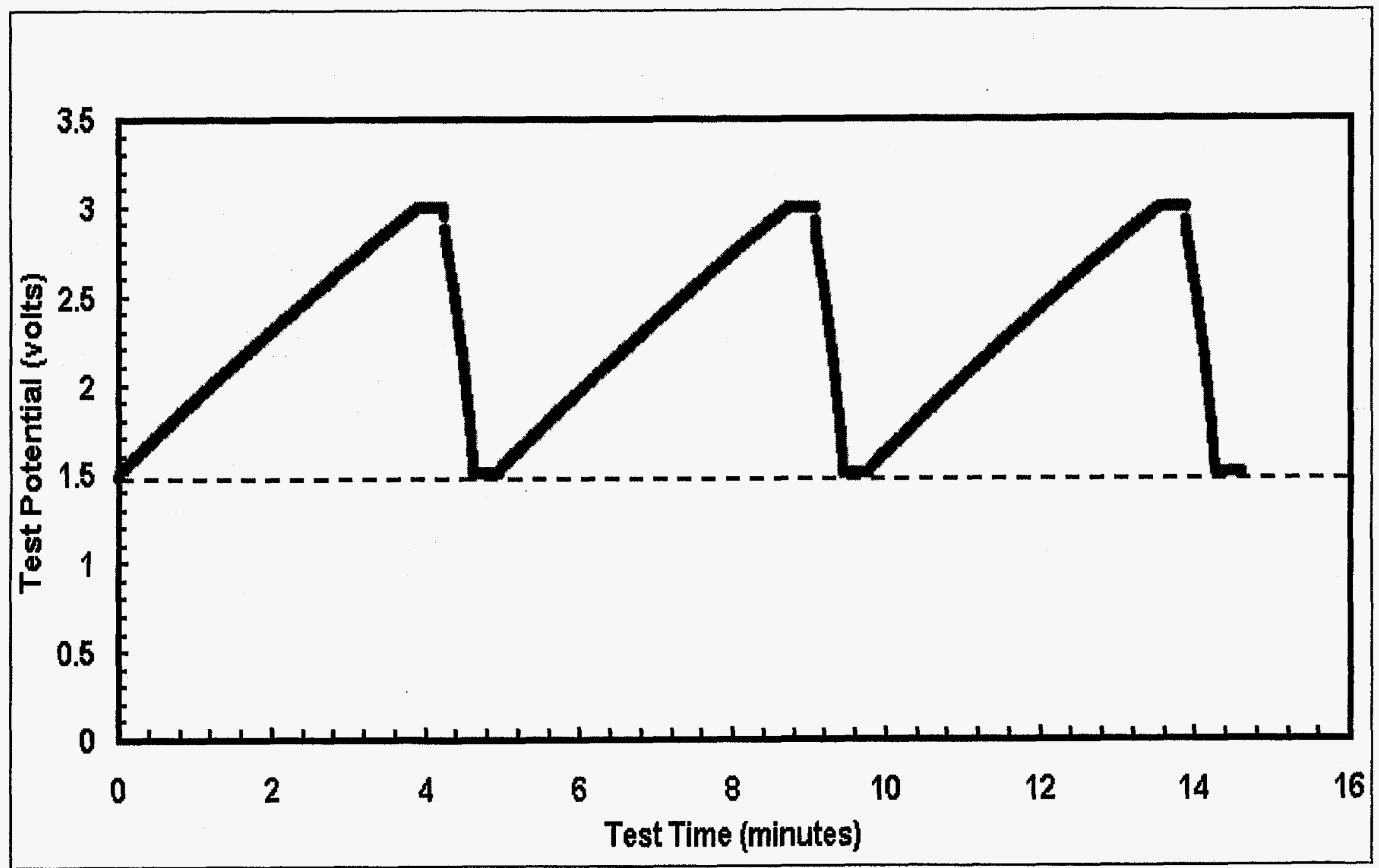

Figure A-9. Test potential as a function of test time for capacitor \#1143 from constant-power test cycle at $1 \mathrm{~A}$ charge and $18 \mathrm{~W}$ discharge. 


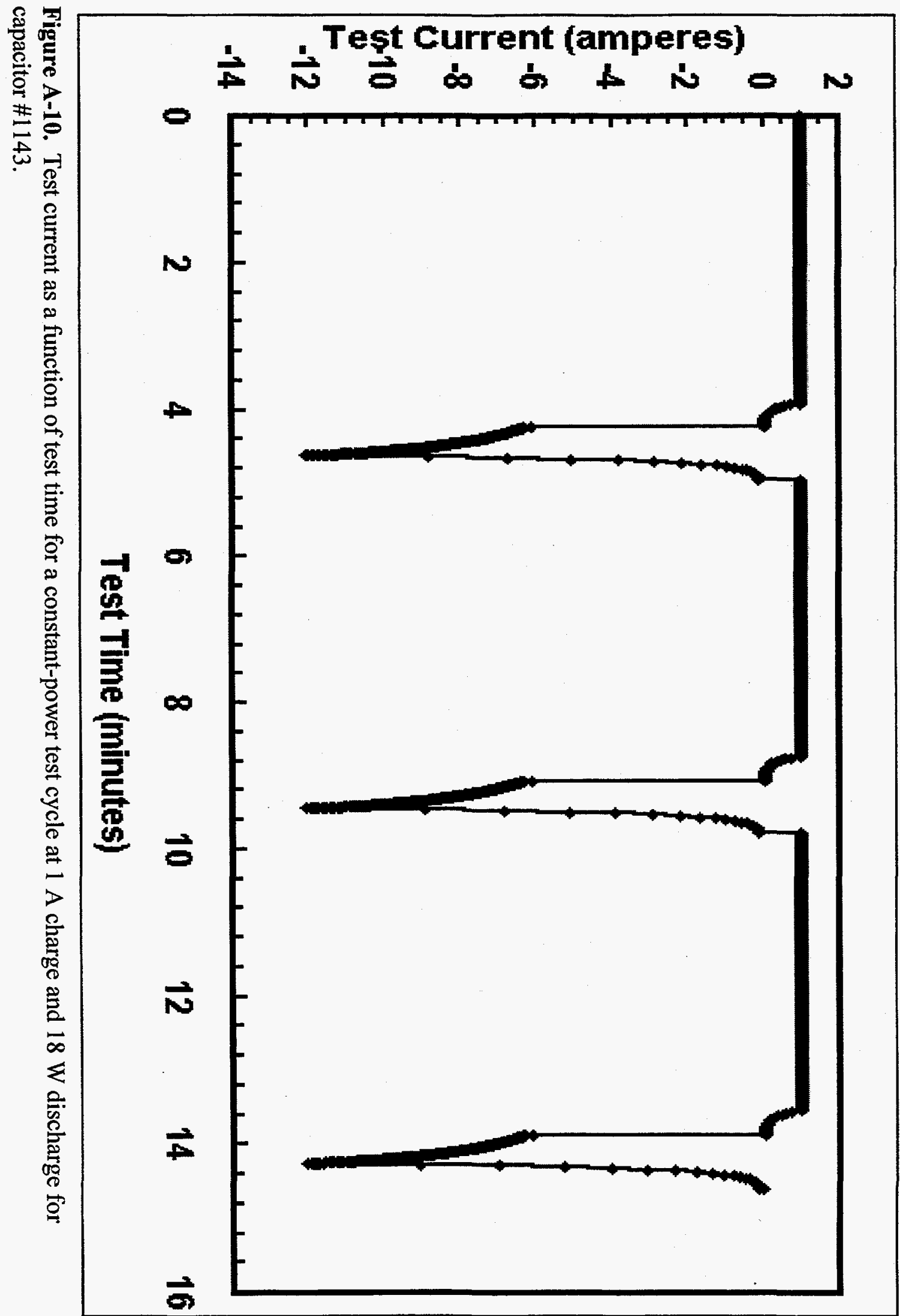




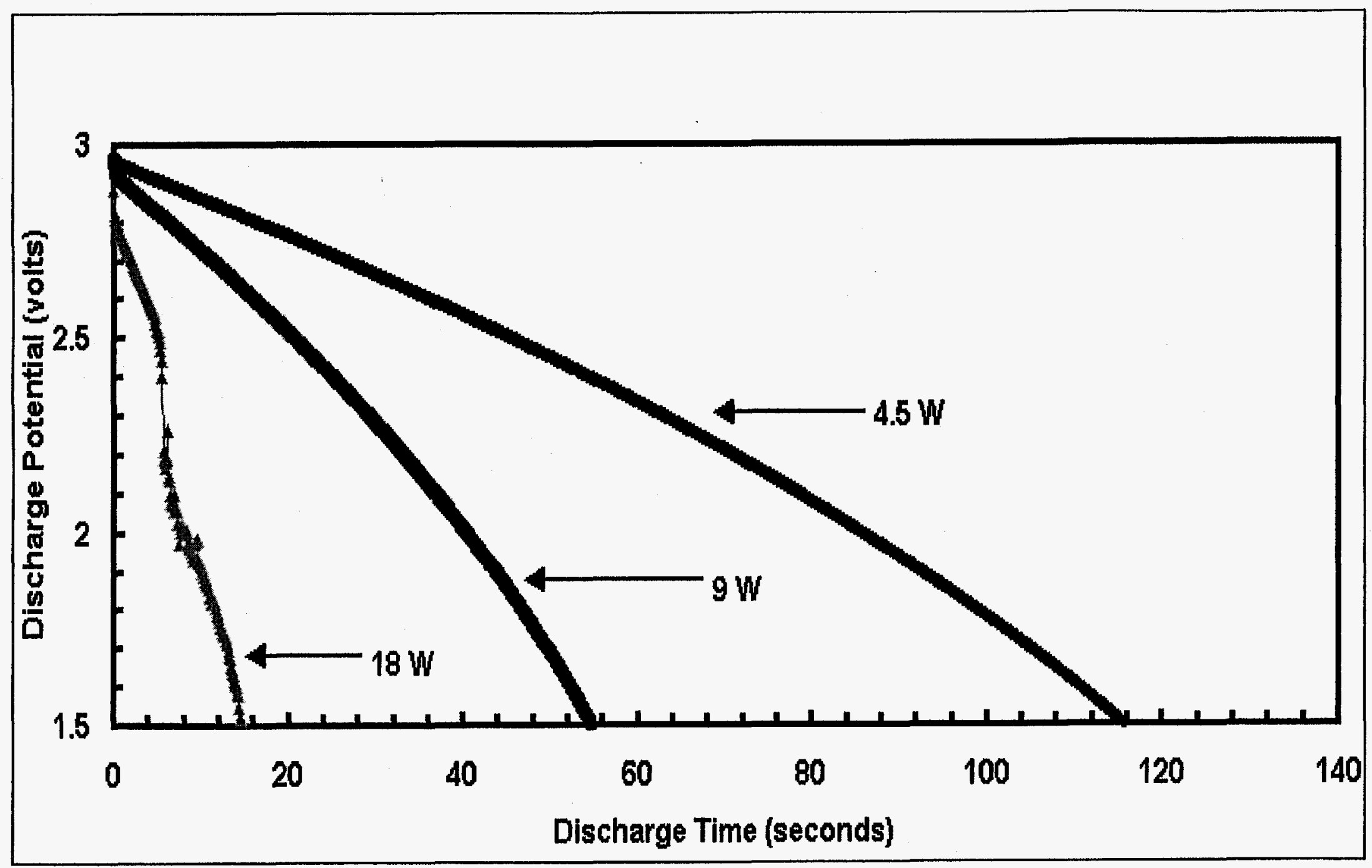

Figure A-11. Data from constant-power test for capacitor \#1141. 


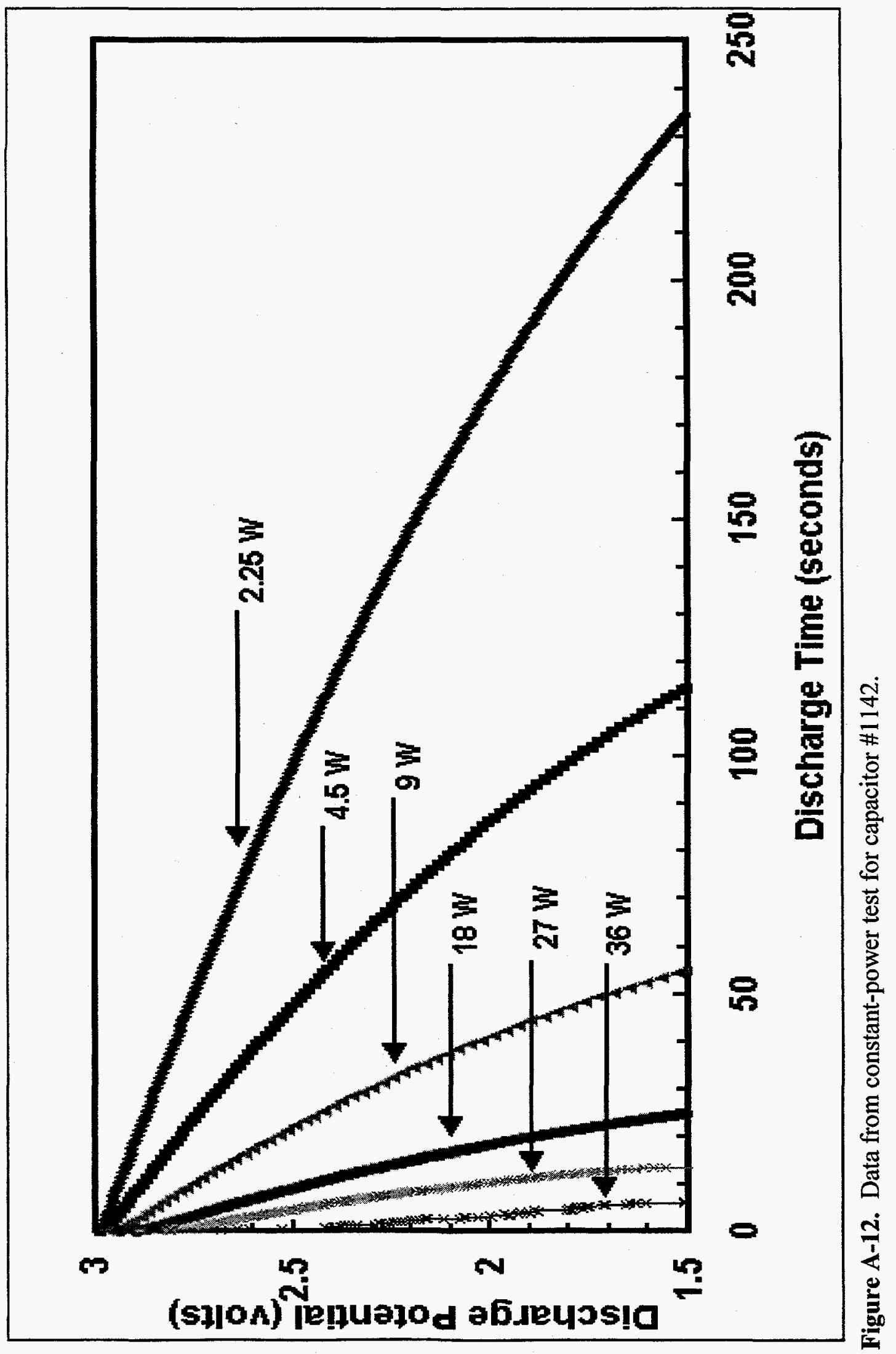




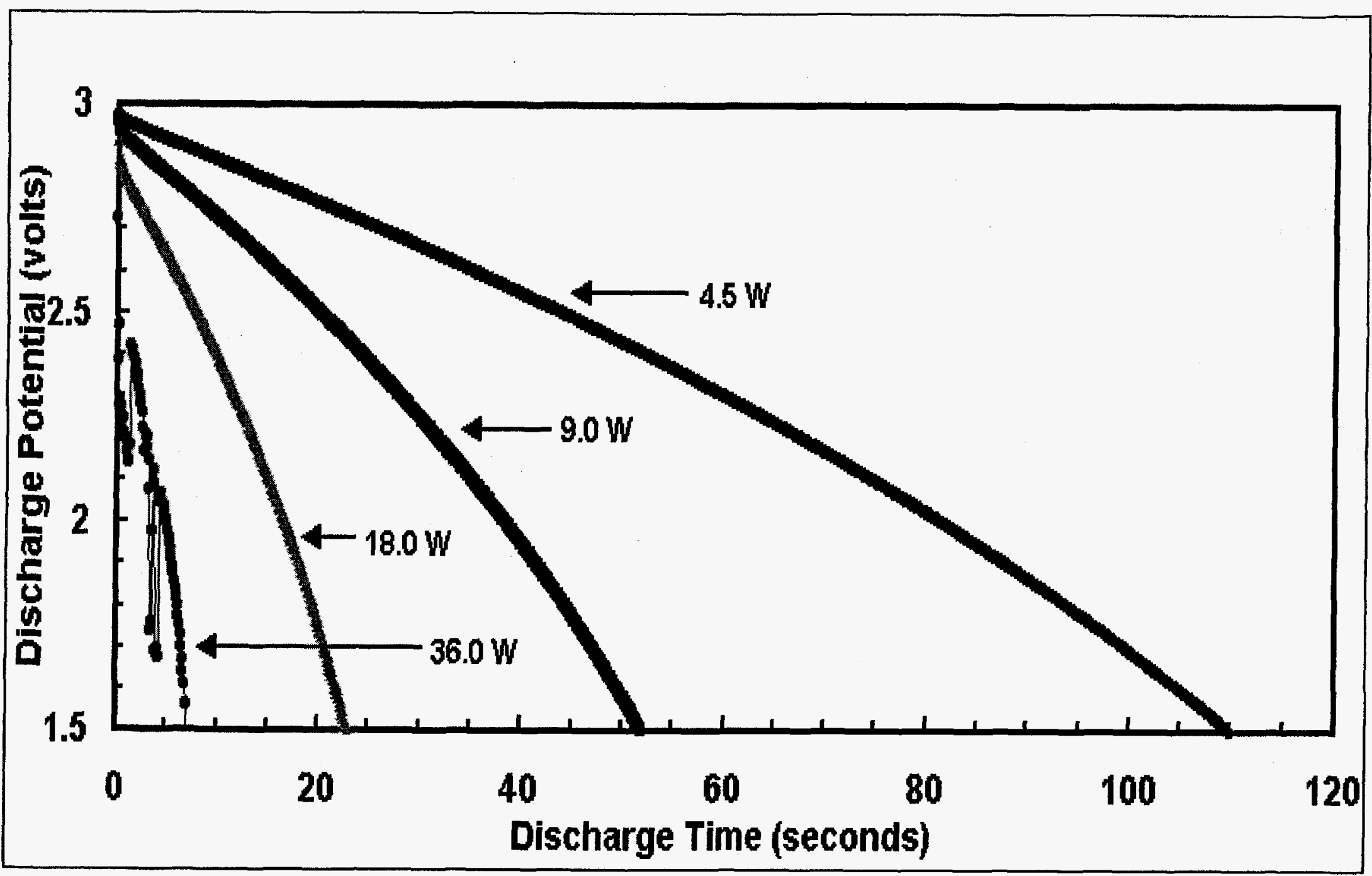

Figure A-13. Data from constant-power test for capacitor \#1143. 


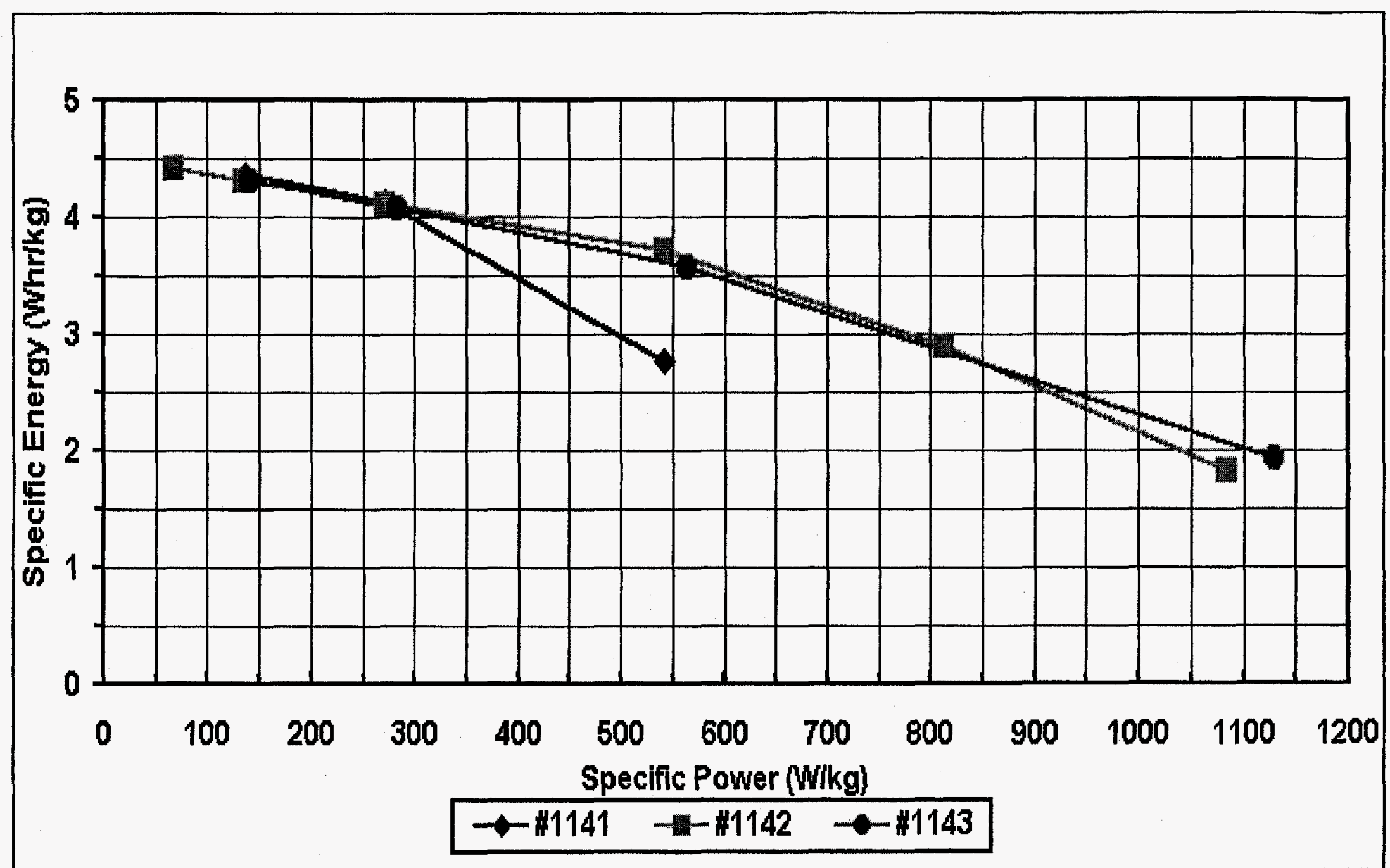

Figure A-14(a). Specific energy as a function of specific power as determined from the constant-power tests using a 3 to $1.5 \mathrm{~V}$ discharge range for capacitors \#1141, \#1142, and \#1143. 


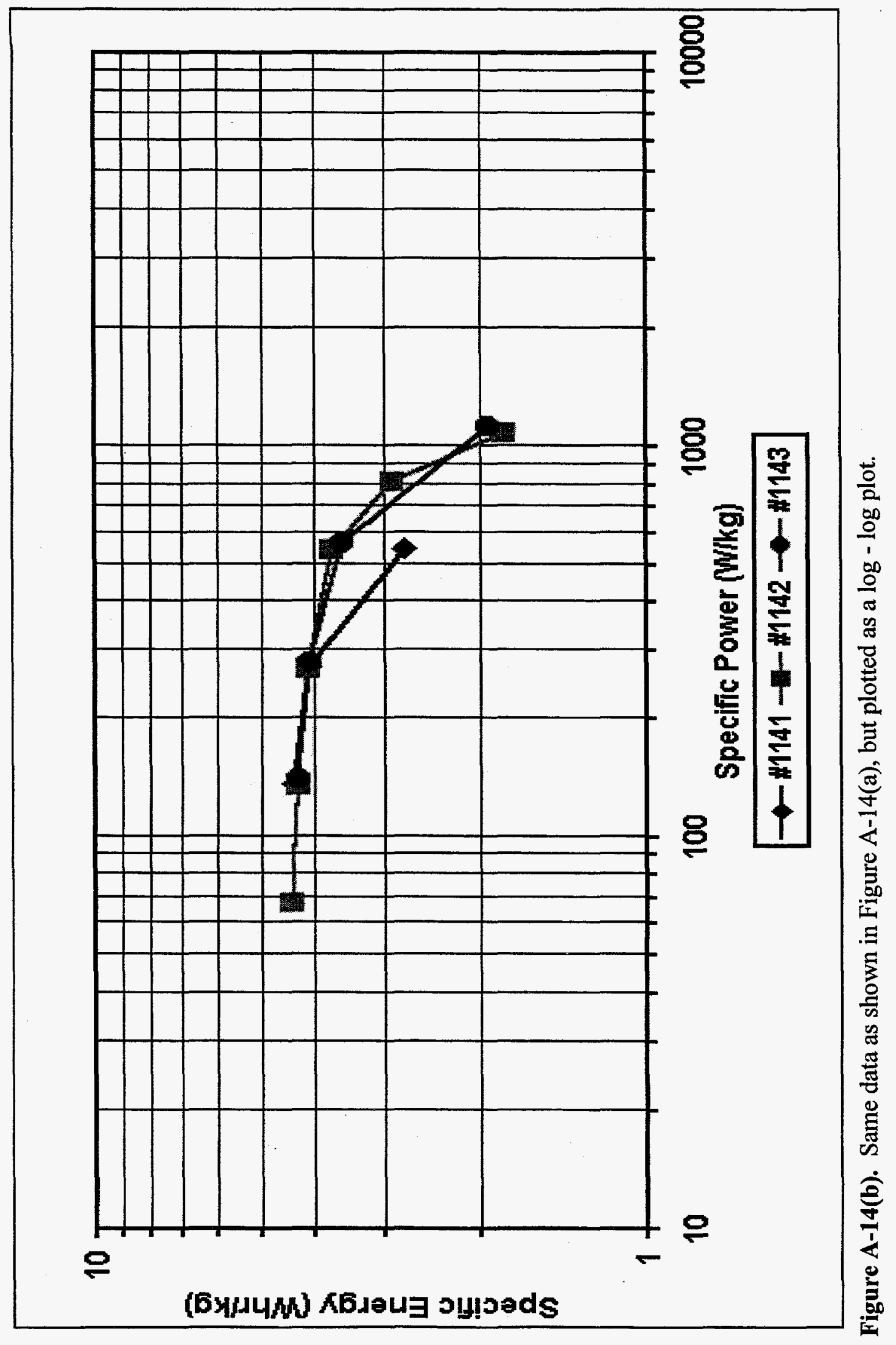




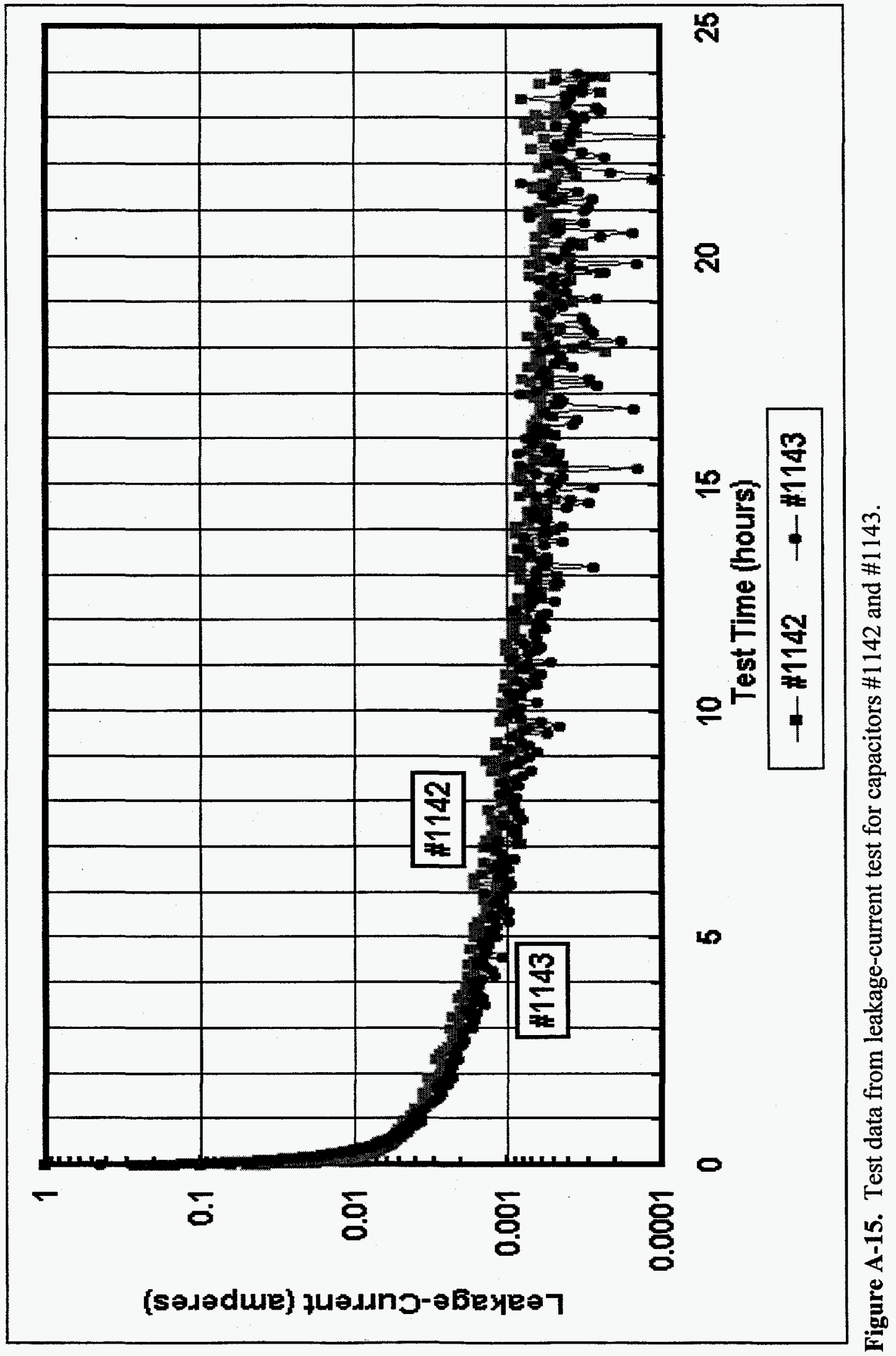




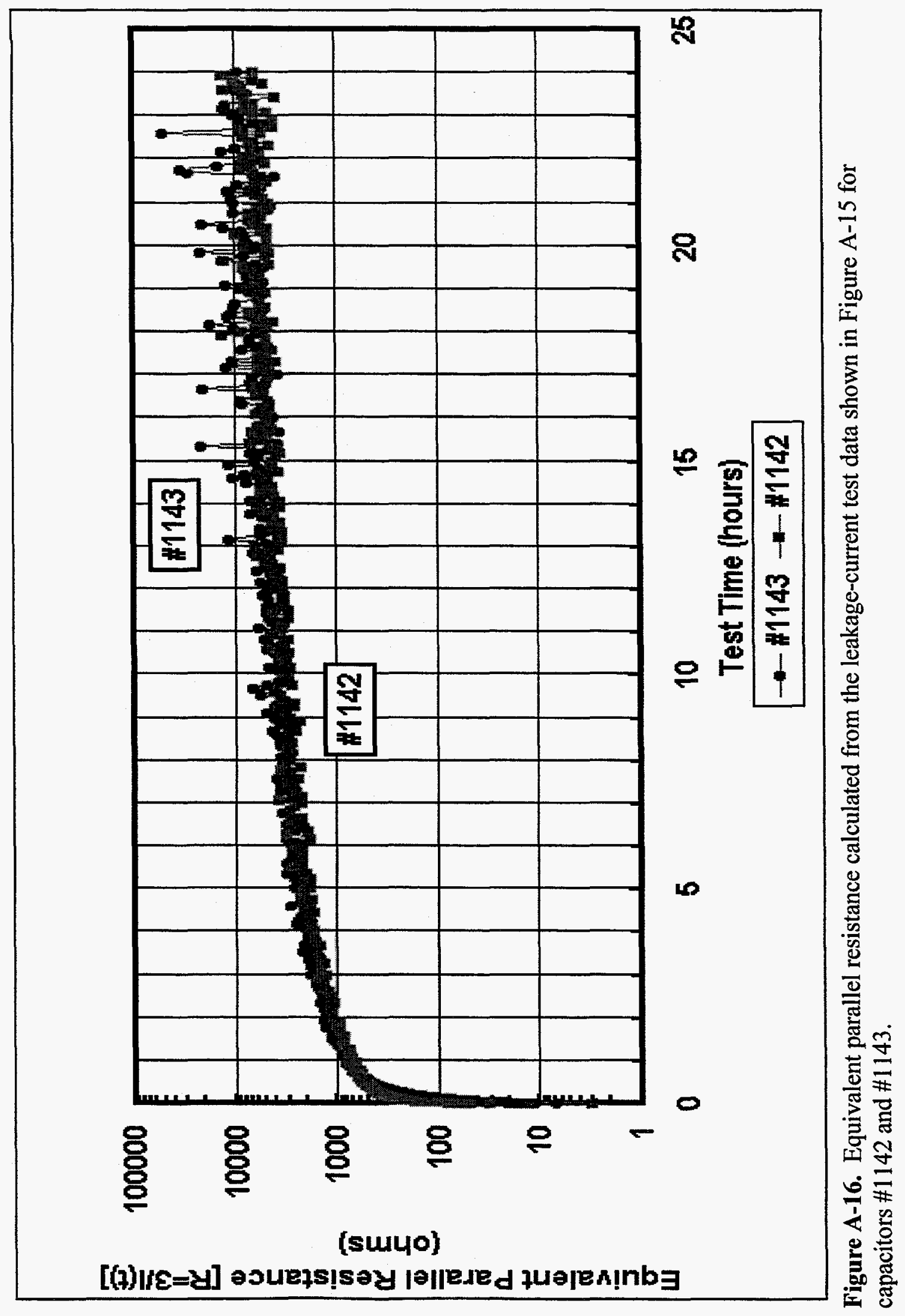




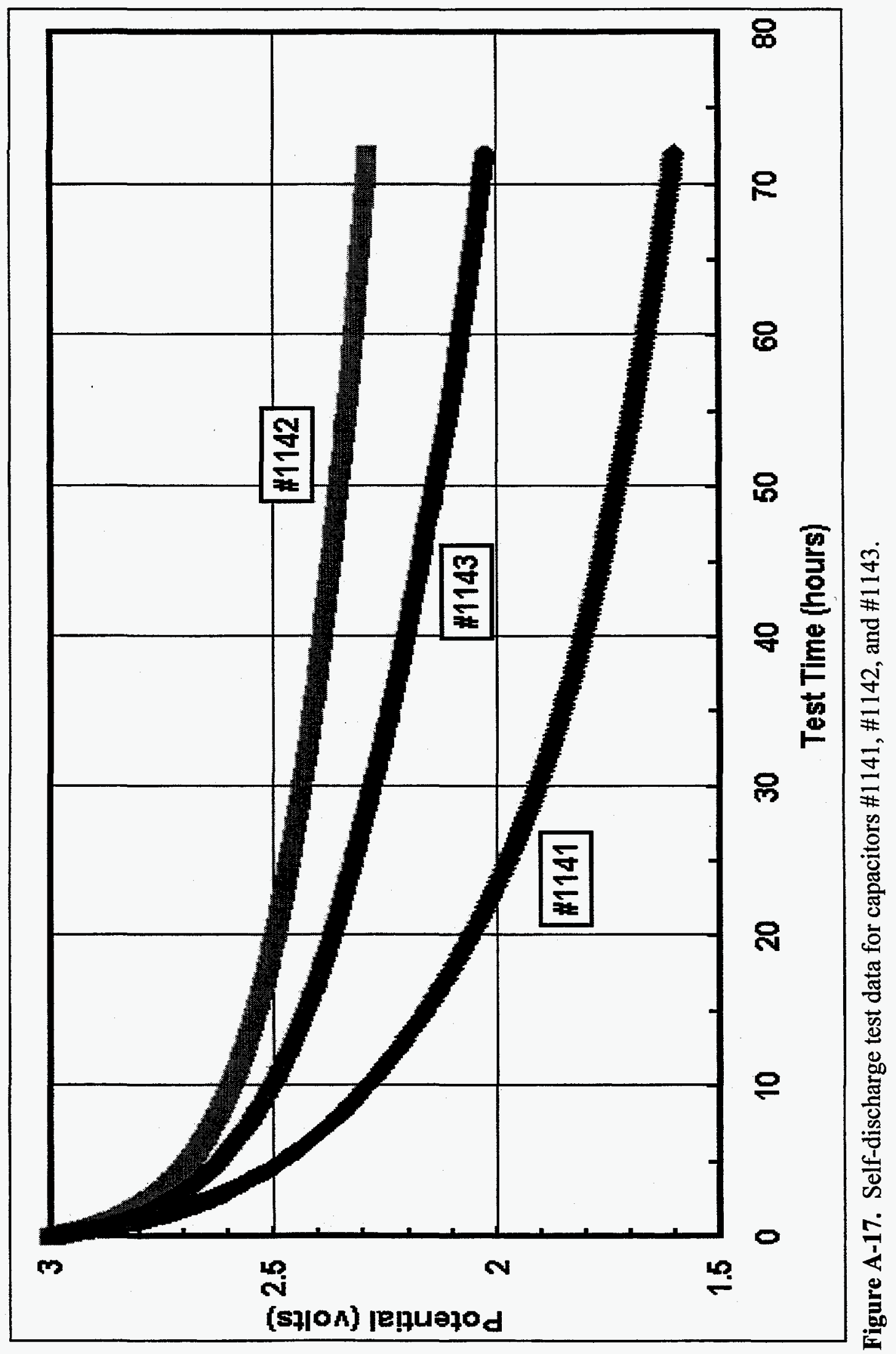




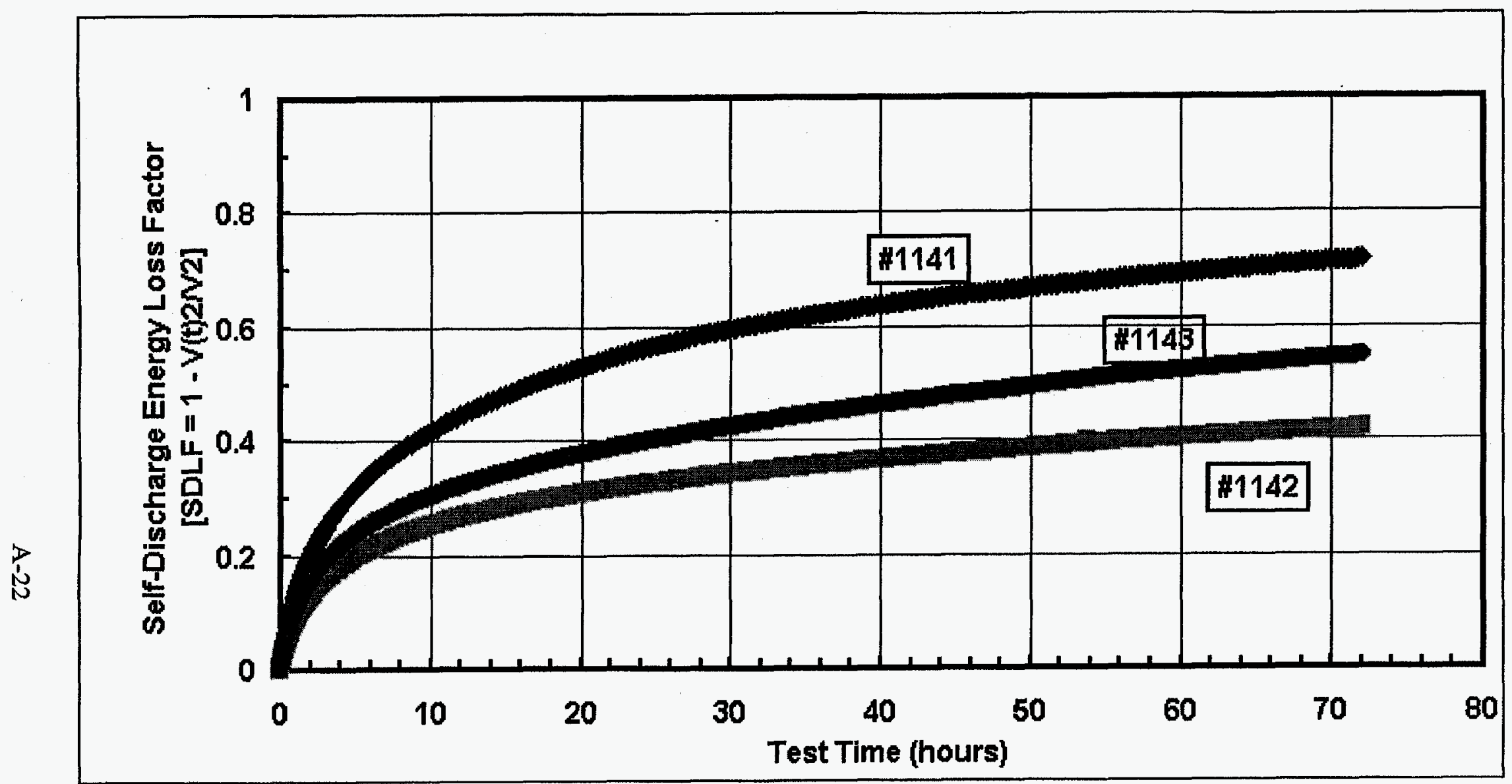

Figure A-18. Self-discharge energy loss factor as a function of test-time calculated from self-discharge test for capacitors \#1141, $\# 1142$, and \#1143. 


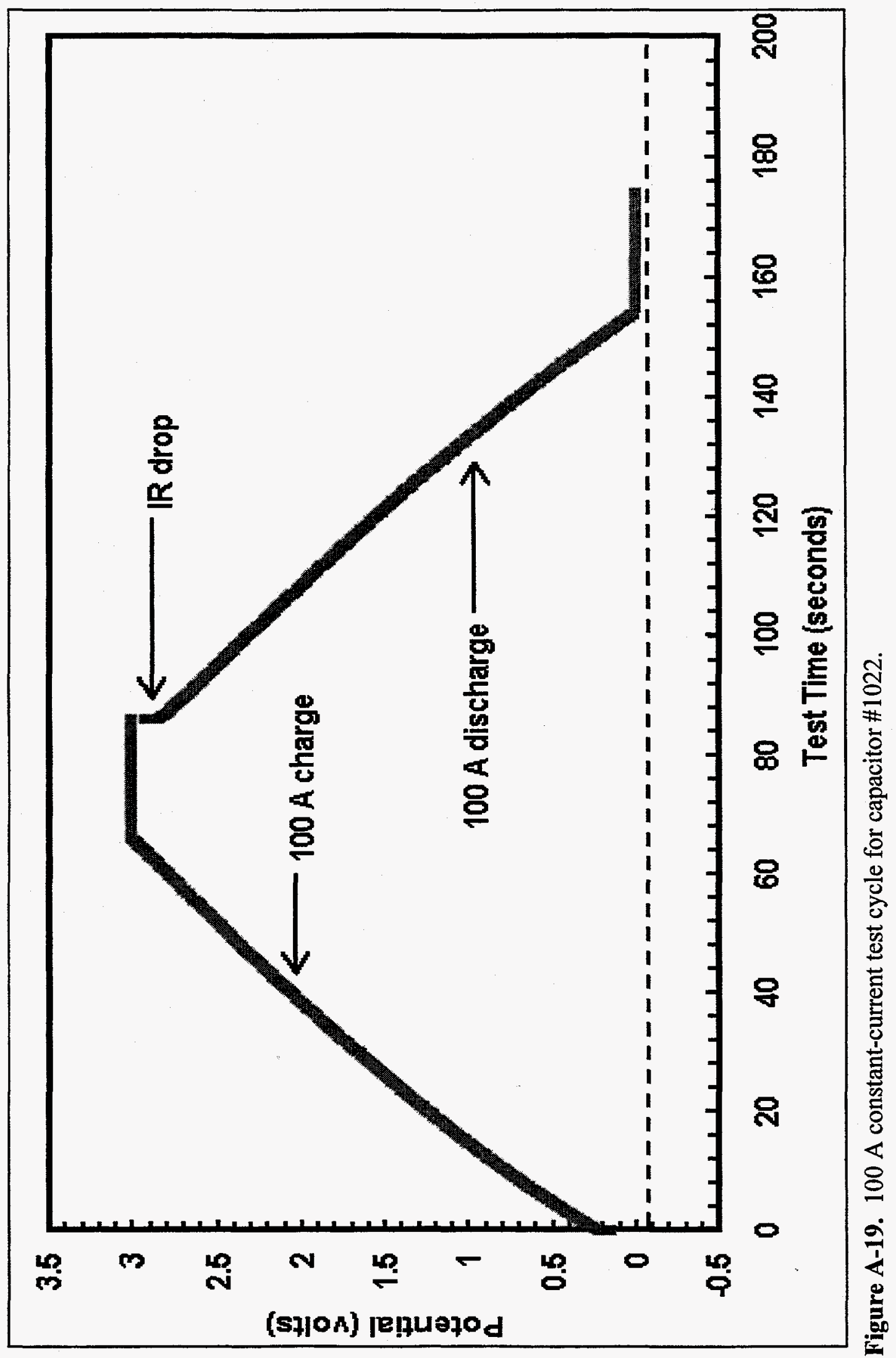




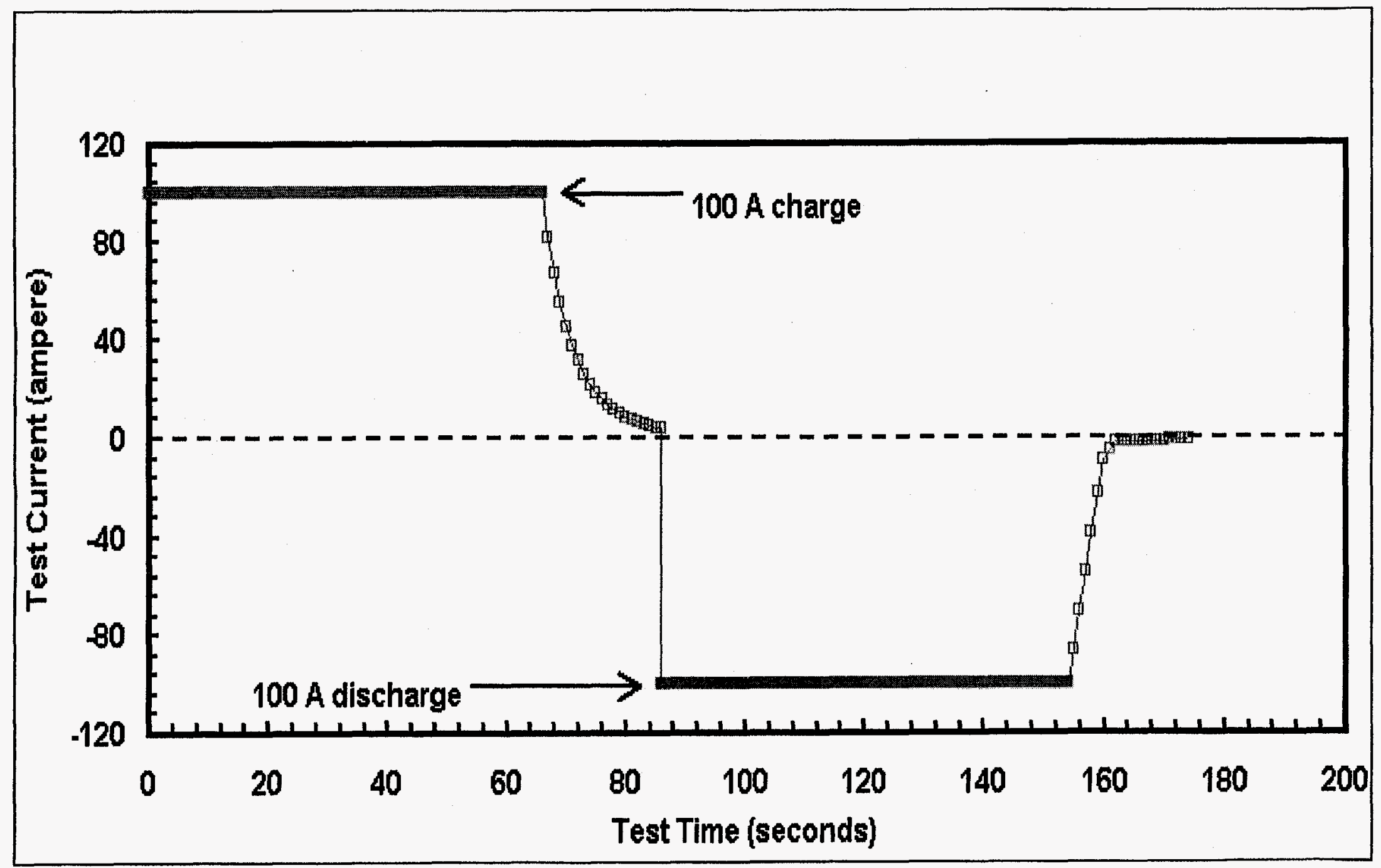

Figure A-20. Constant-current test cycle at 100 A for capacitor \#1022. 


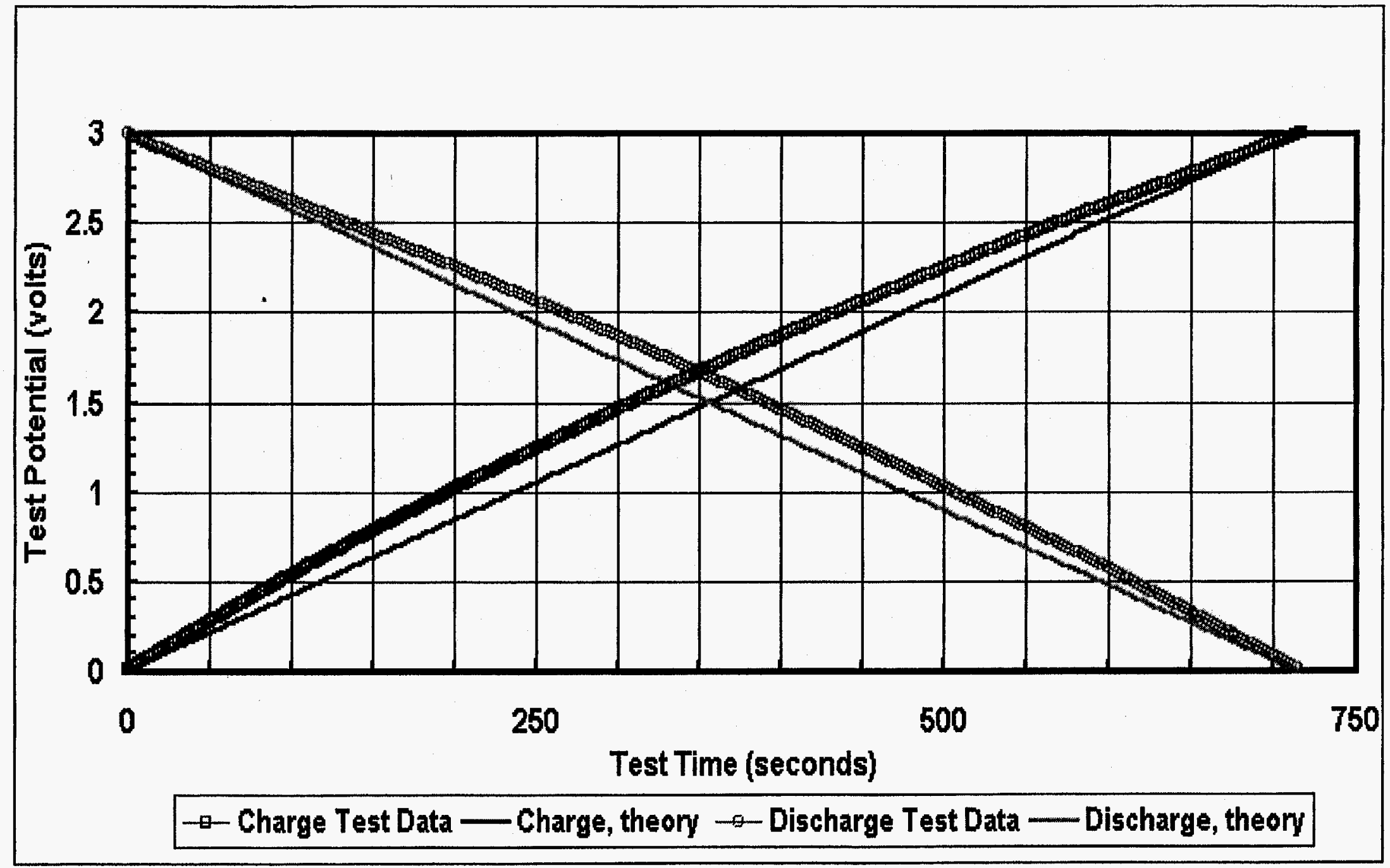

Figure A-21. Comparison between the experimental and theoretical ideal capacitor voltage as a function of test time for a $10 \mathrm{~A}$ constant-current charge and discharge test for capacitor \#1021. 


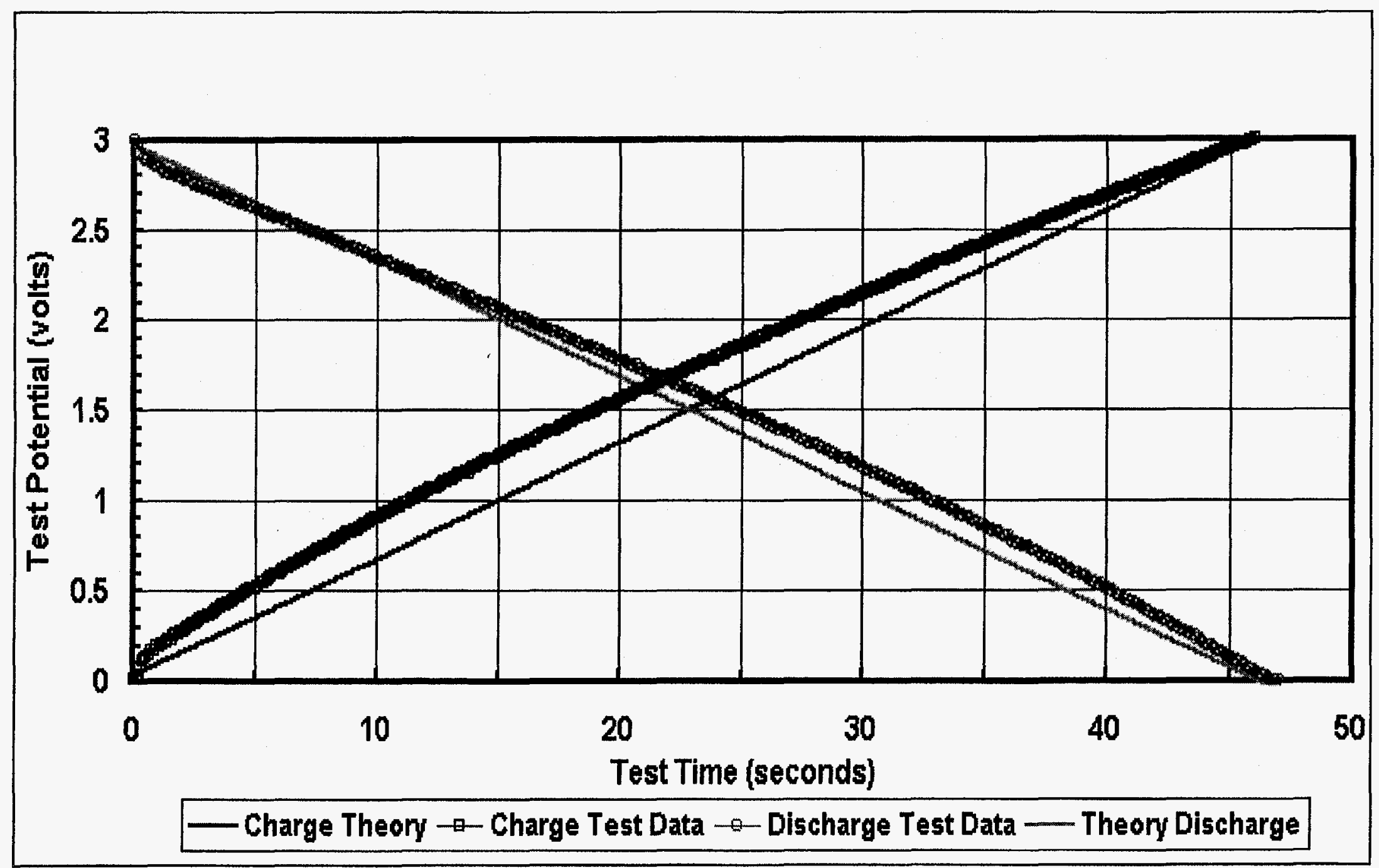

Figure A-22. Same as Figure A-21, except for a 100 A constant-current charge and discharge test. 


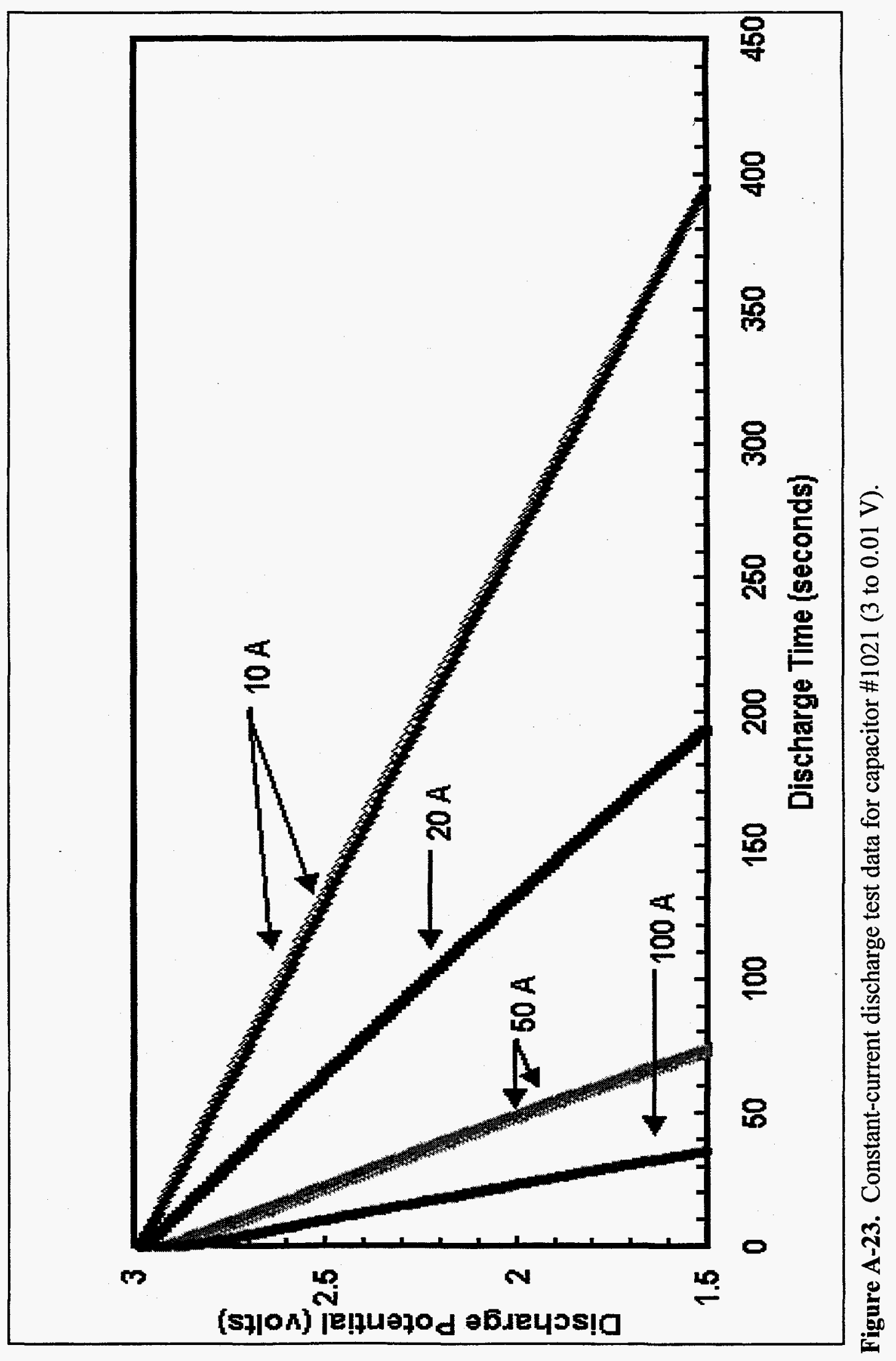




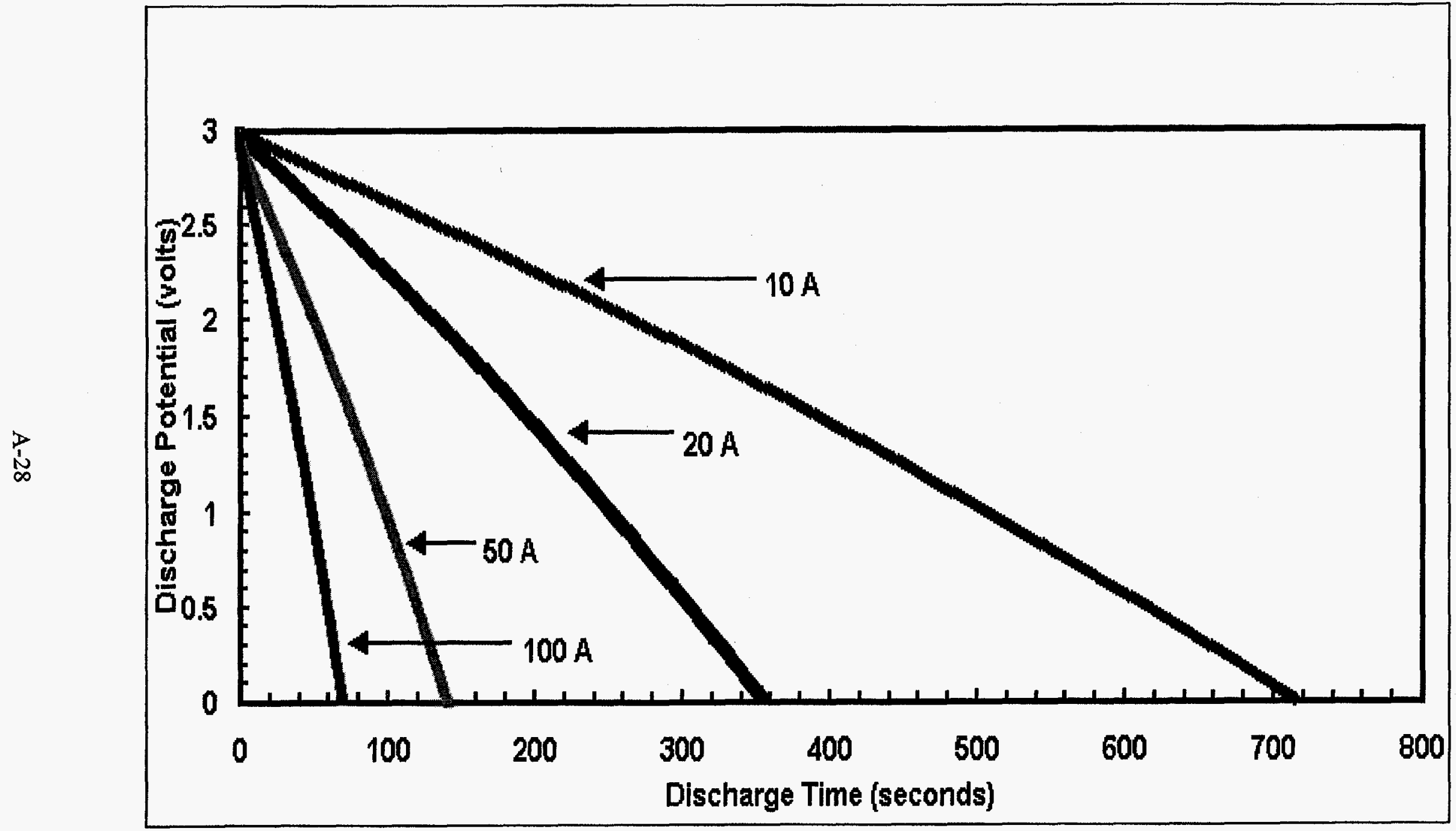

Figure A-24. Data from constant-current discharge test for capacitor \#1021 (3 to $0.01 \mathrm{~V}$ ). 


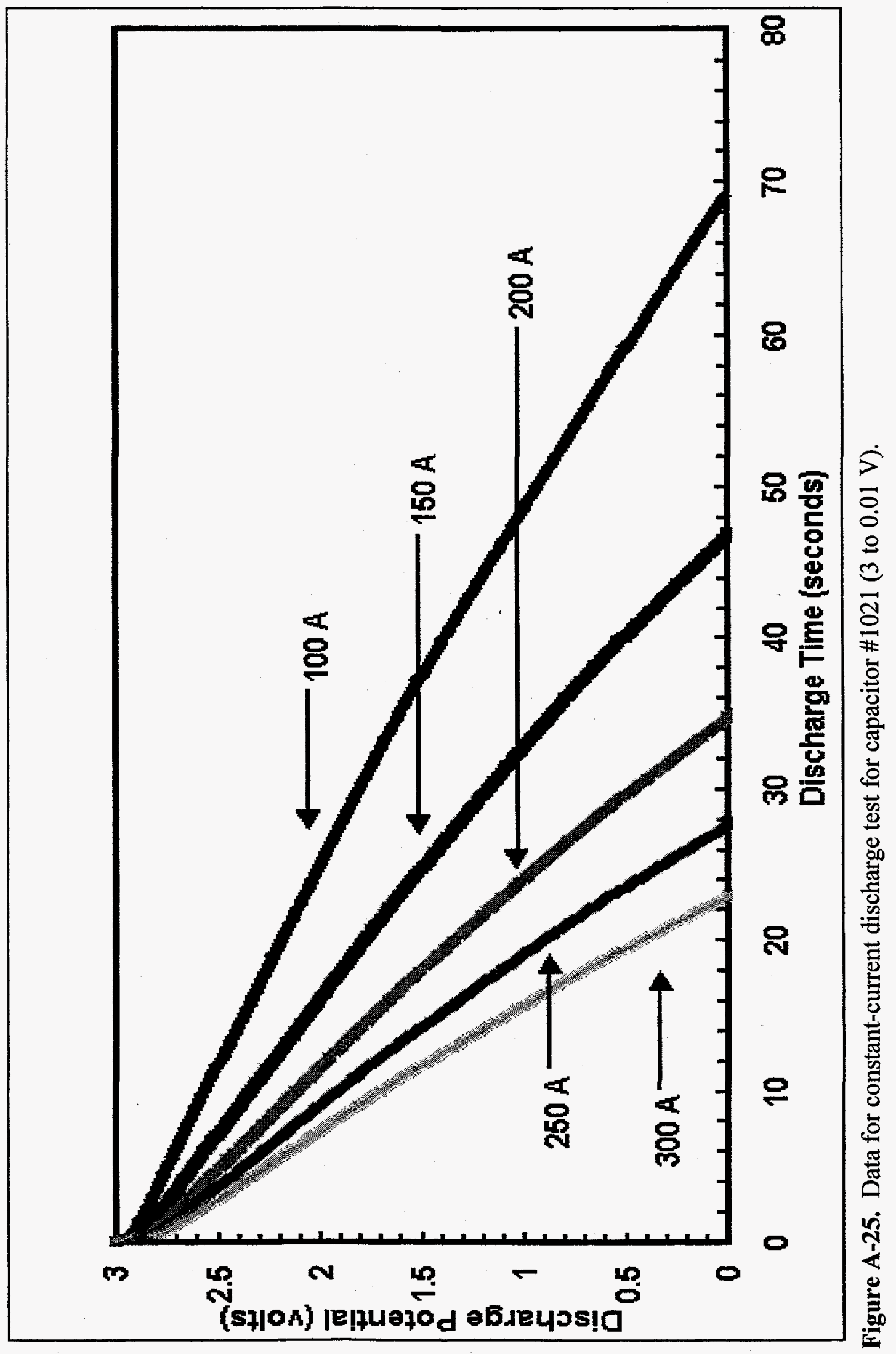




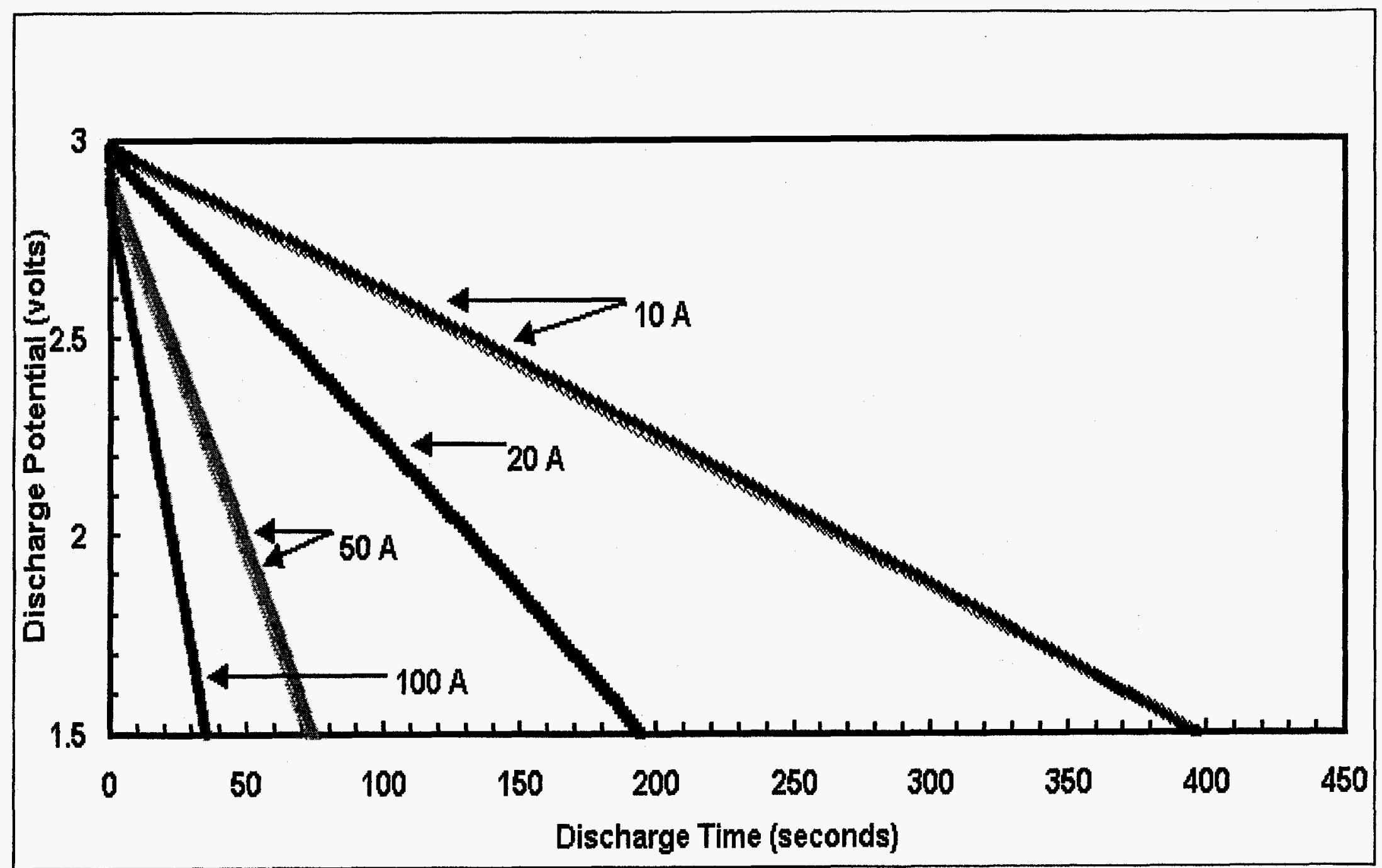

Figure A-26. Constant-current discharge test data for capacitor \#1022 (3 to $1.5 \mathrm{~V}$ ). 


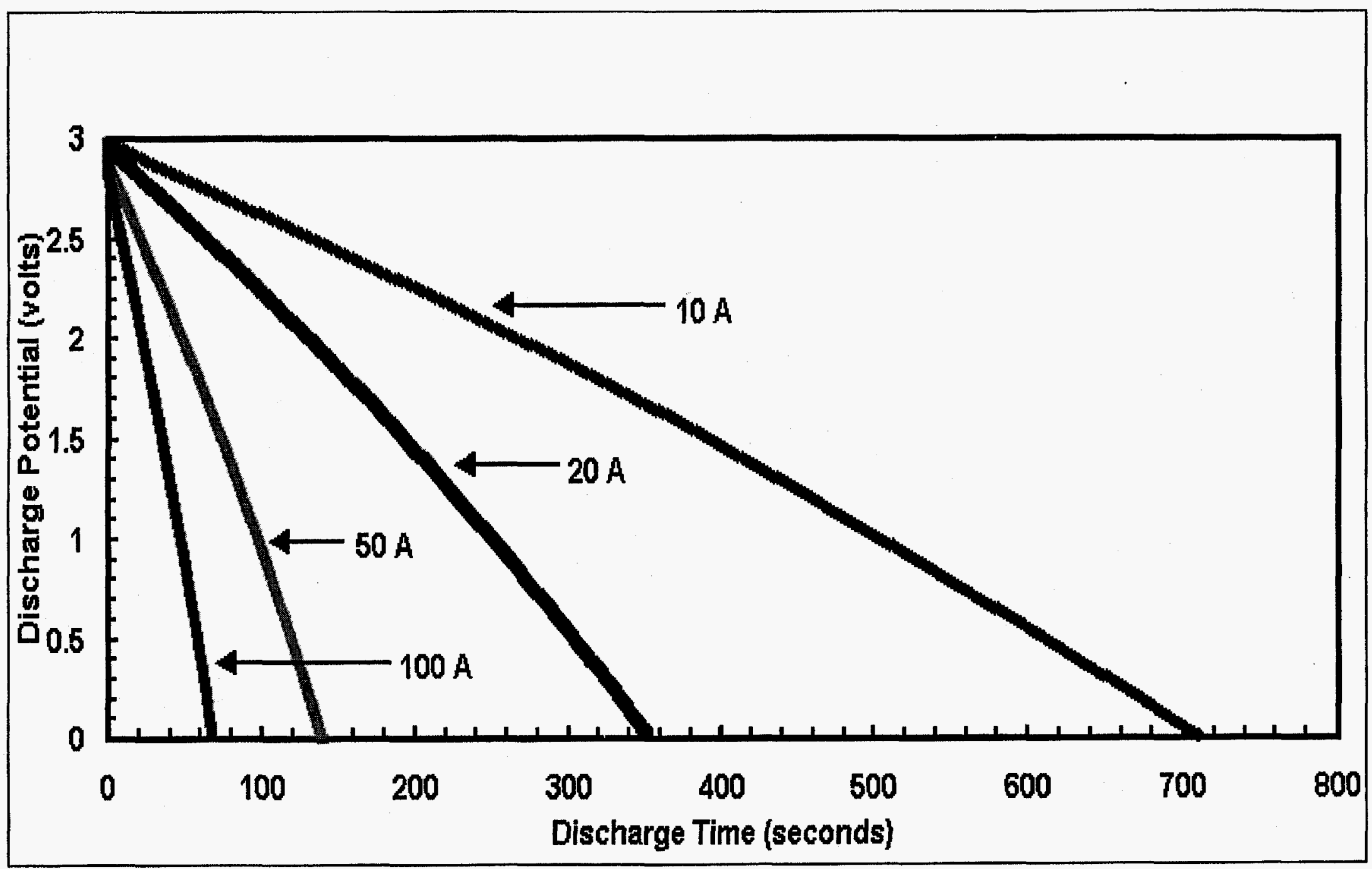

Figure A-27. Constant-current discharge test data for capacitor \#1022 (3 to $0.01 \mathrm{~V}$ ). 


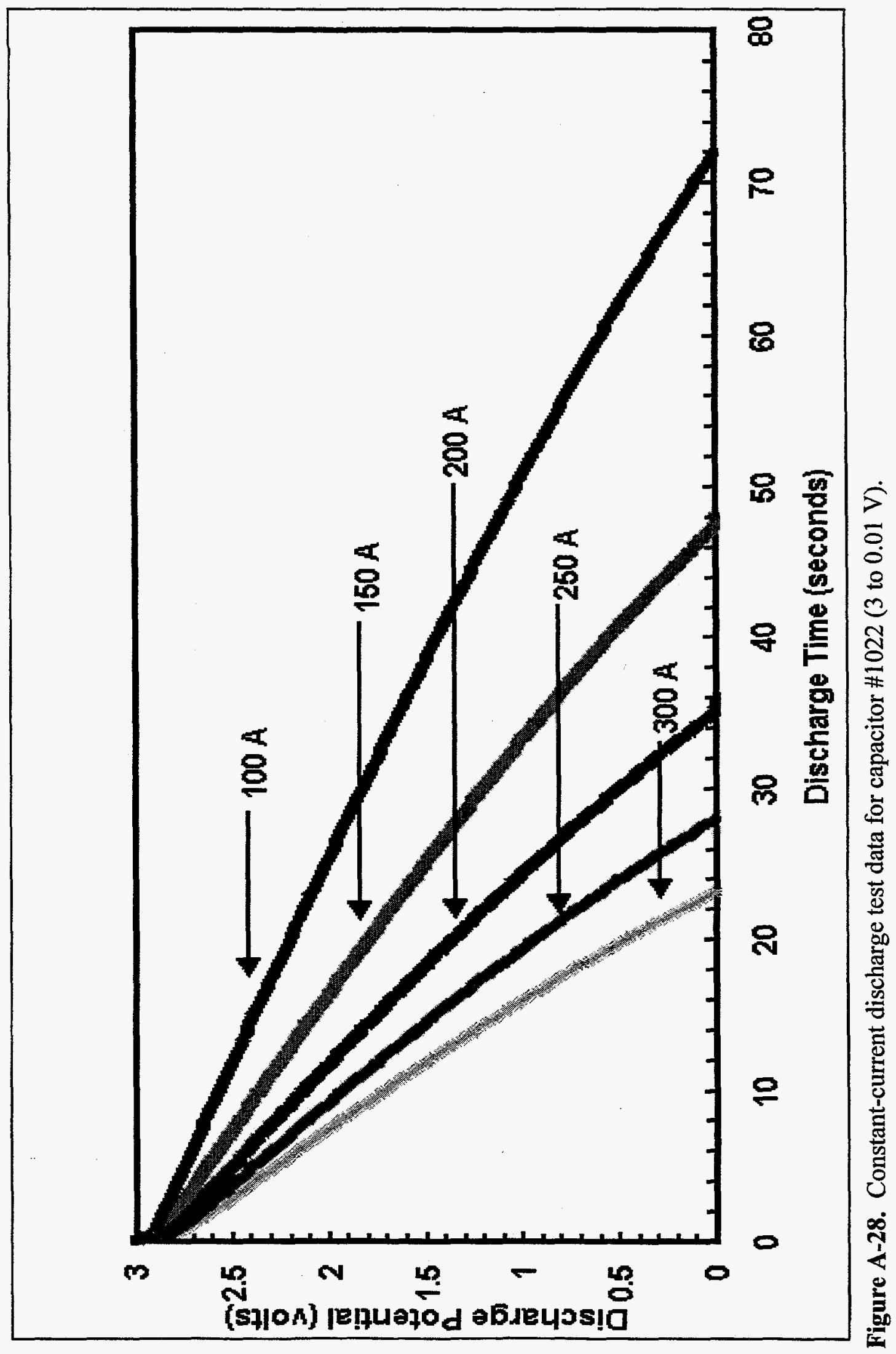




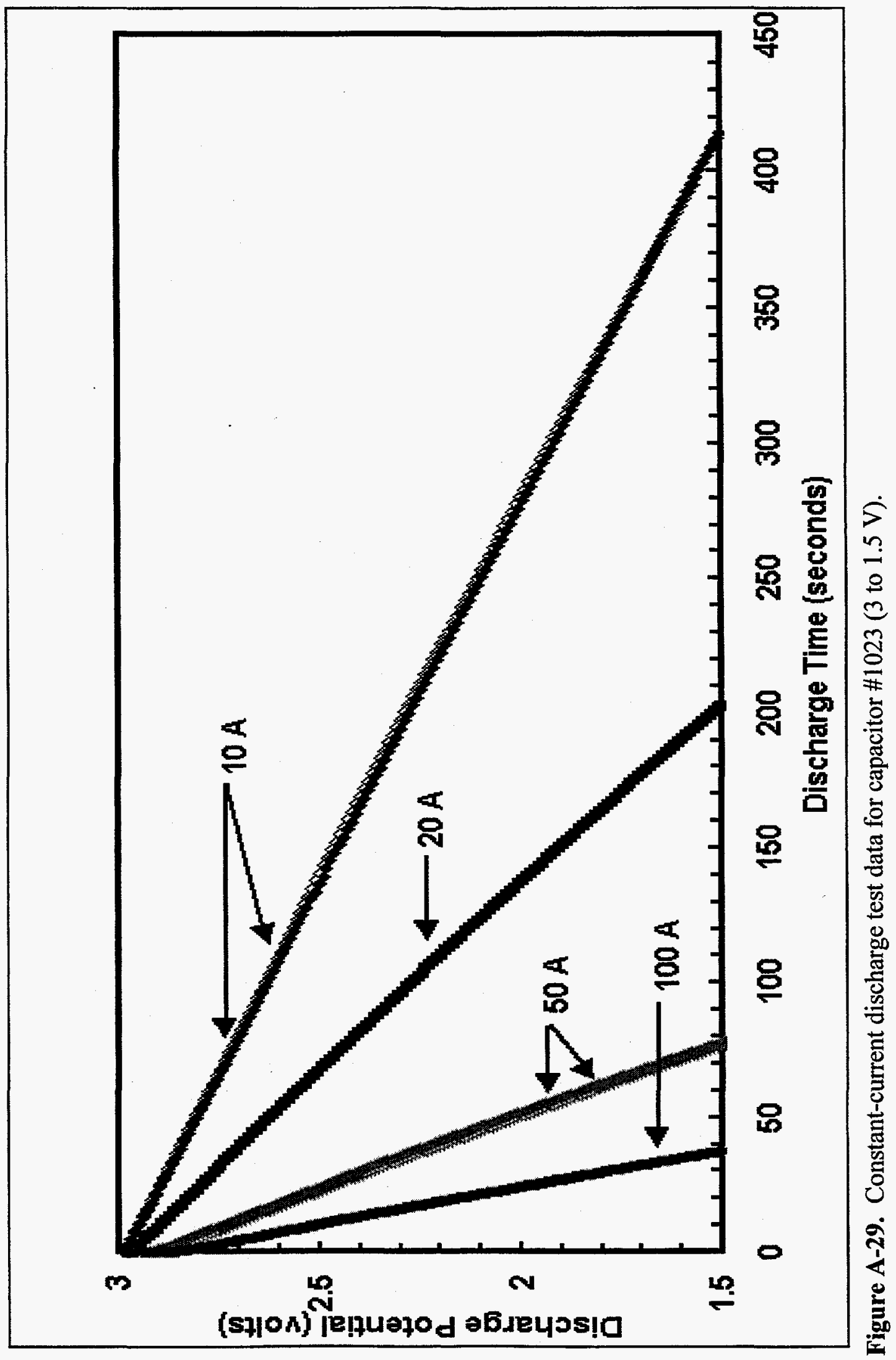




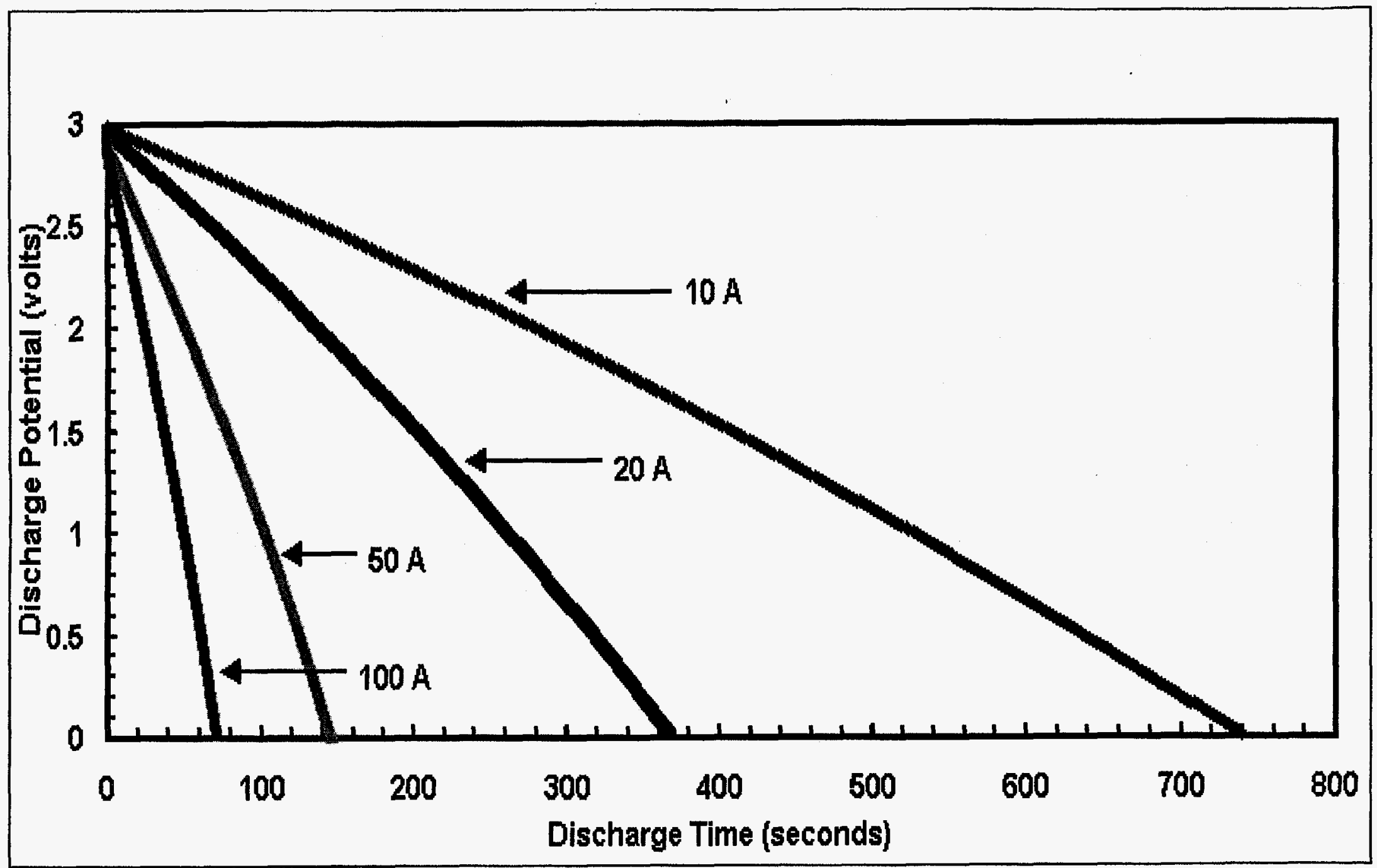

Figure A-30. Data from constant-current discharge test for capacitor \#1023 (3 to $0.01 \mathrm{~V}$ ). 


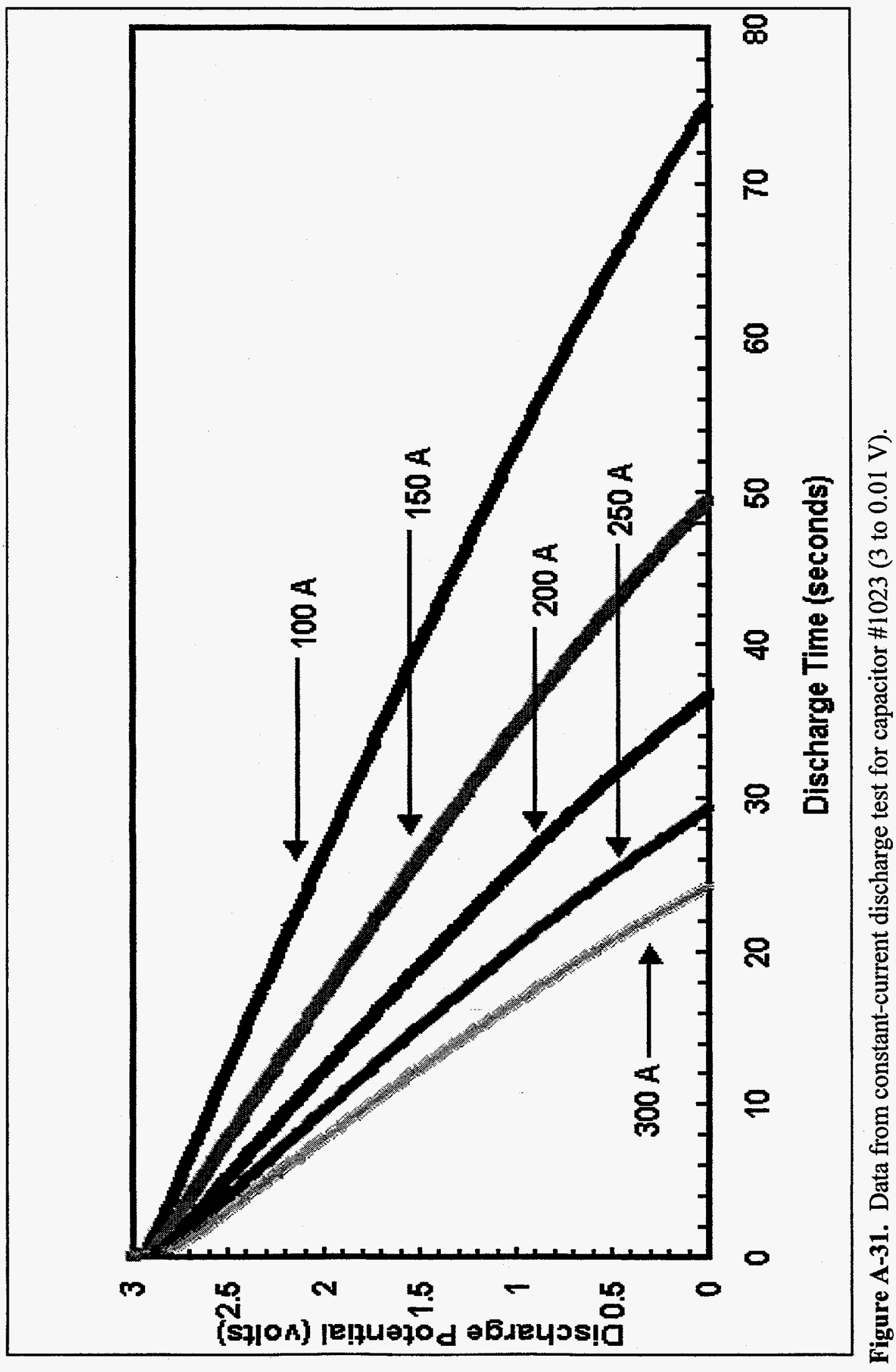




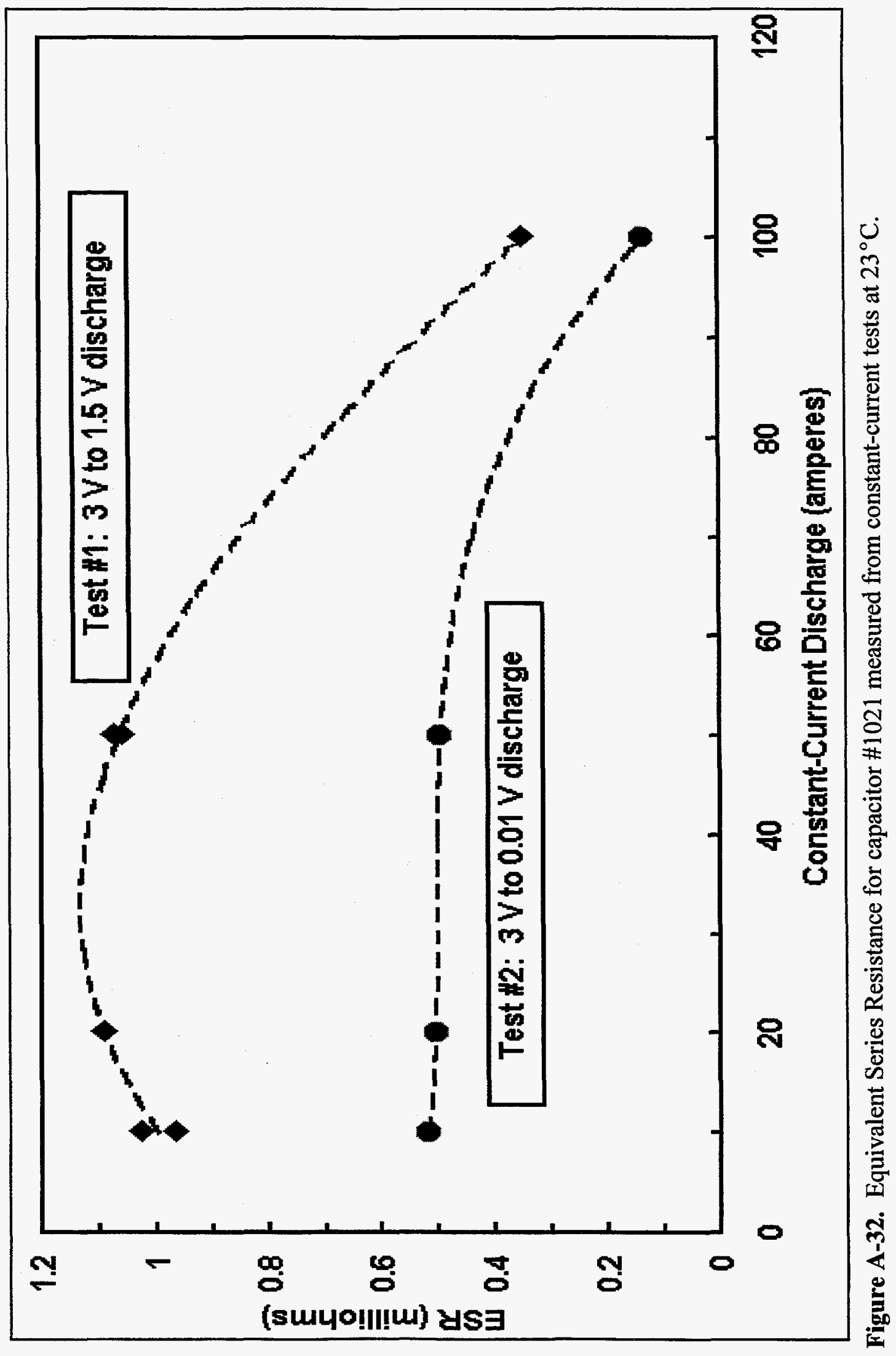




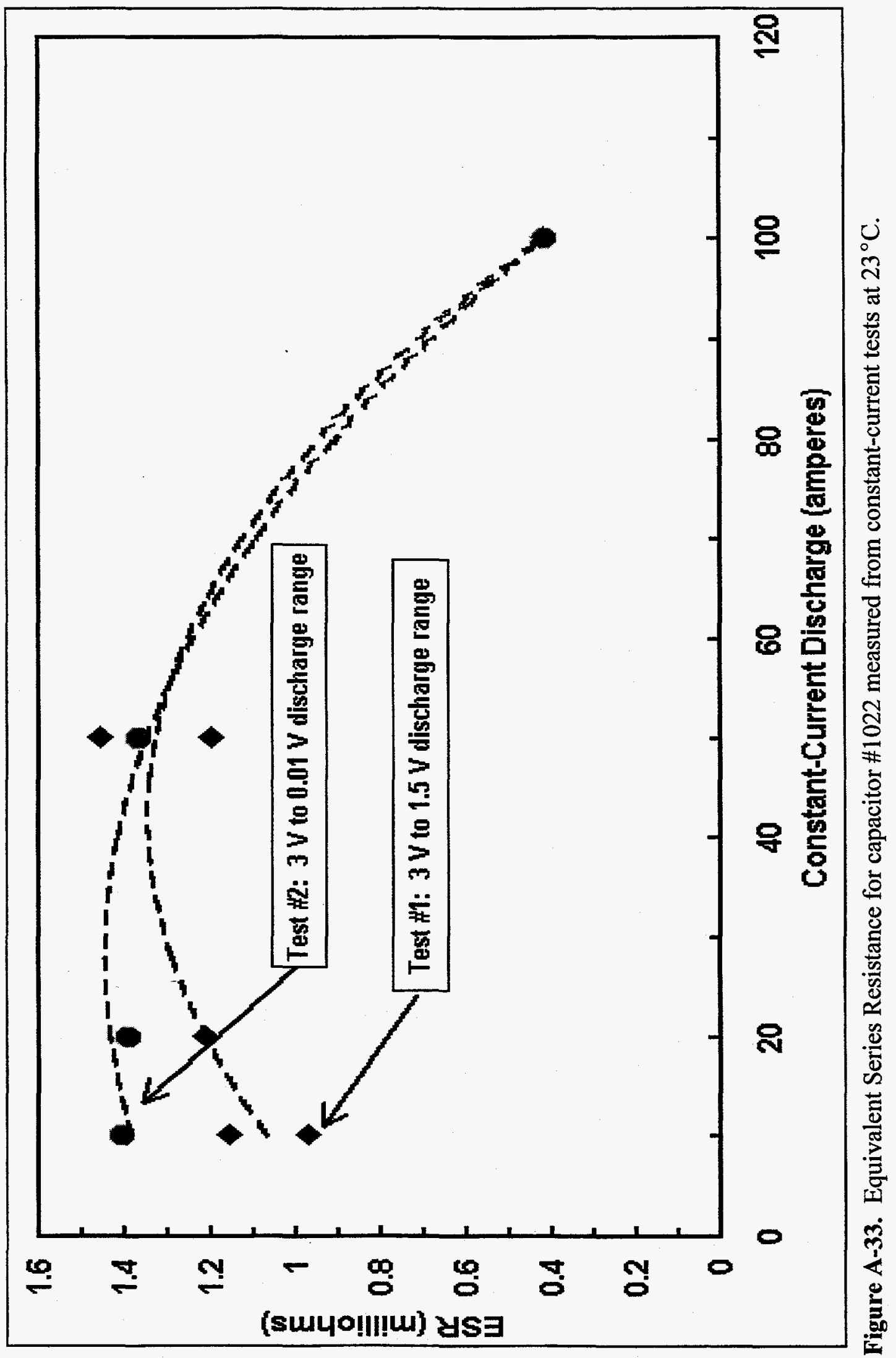




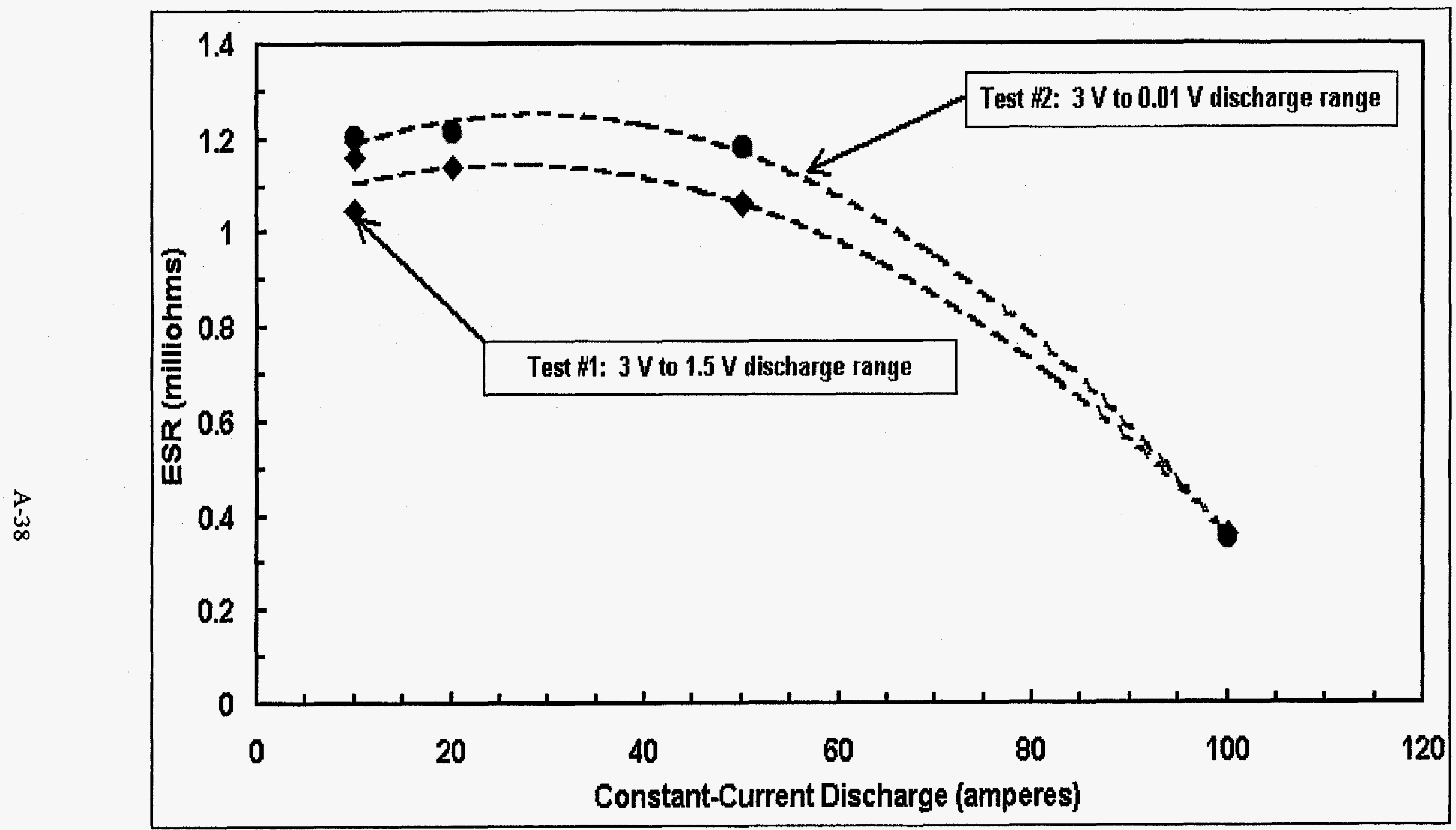

Figure A-34. Equivalent Series Resistance for capacitor $\# 1023$ measured from constant-current tests at $23^{\circ} \mathrm{C}$. 


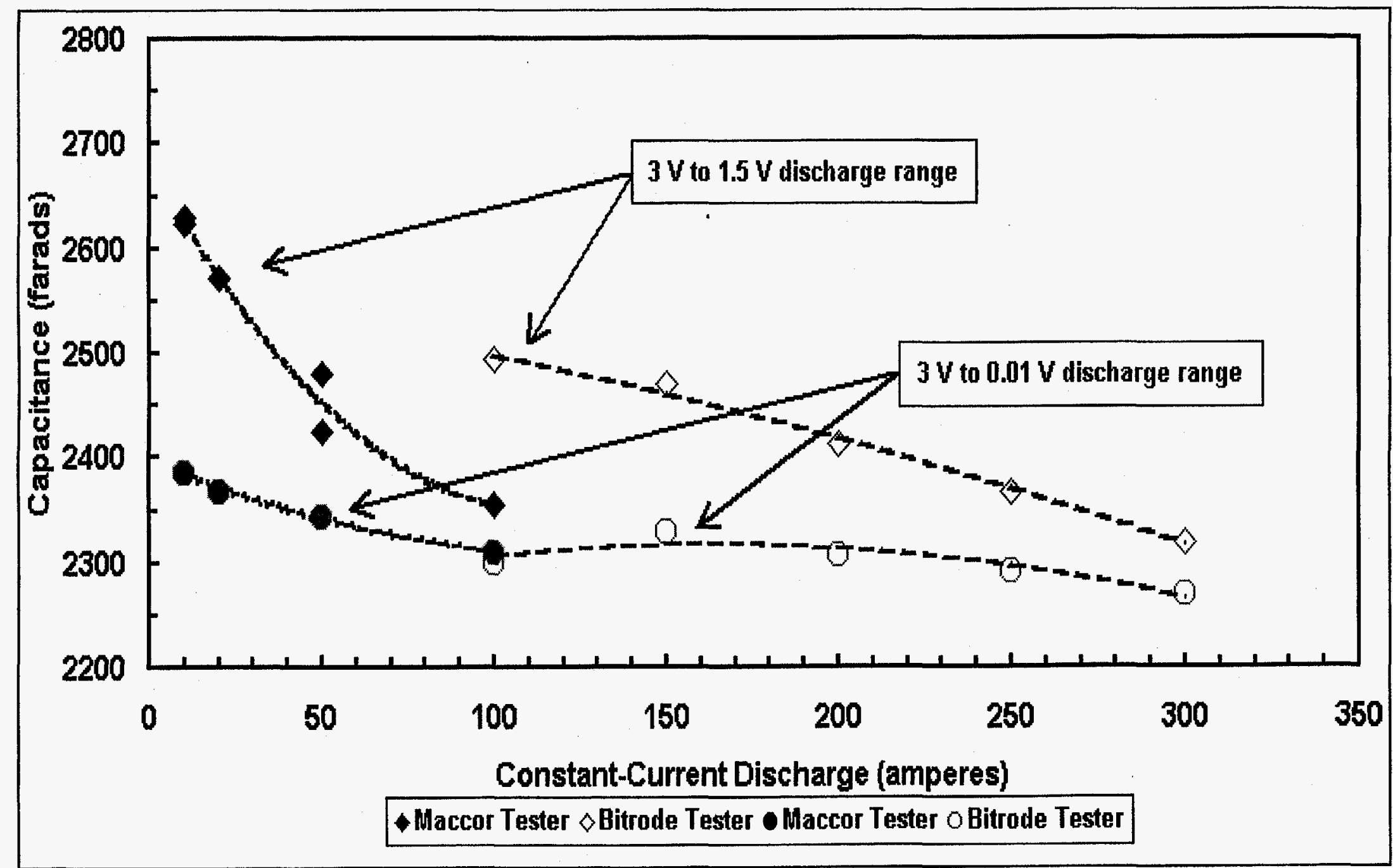

Figure A-35. Measured capacitance for capacitor $\# 1021$ at $23^{\circ} \mathrm{C}$. 


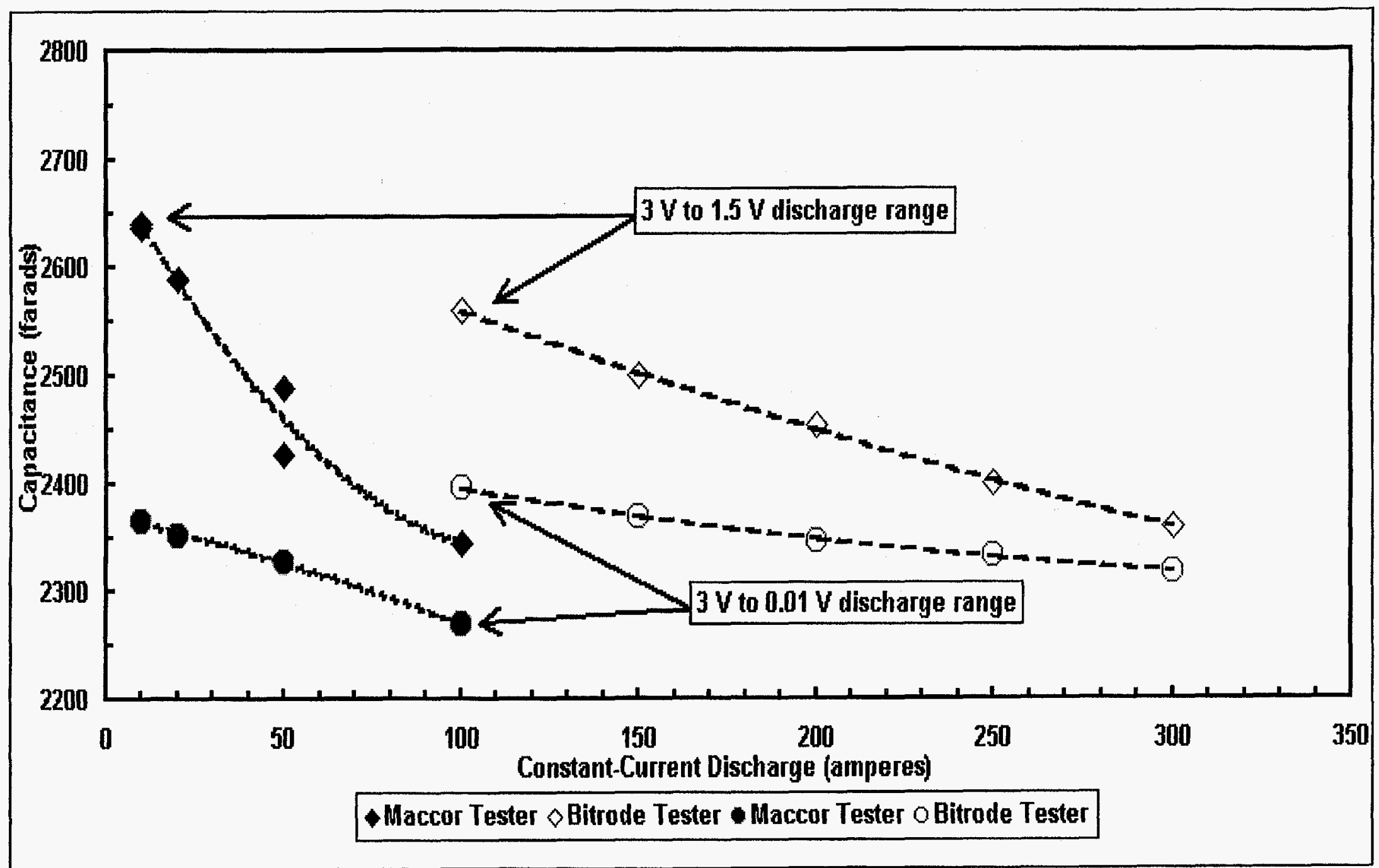

Figure A-36. Measured capacitance for capacitor $\# 1022$ at $23^{\circ} \mathrm{C}$. 


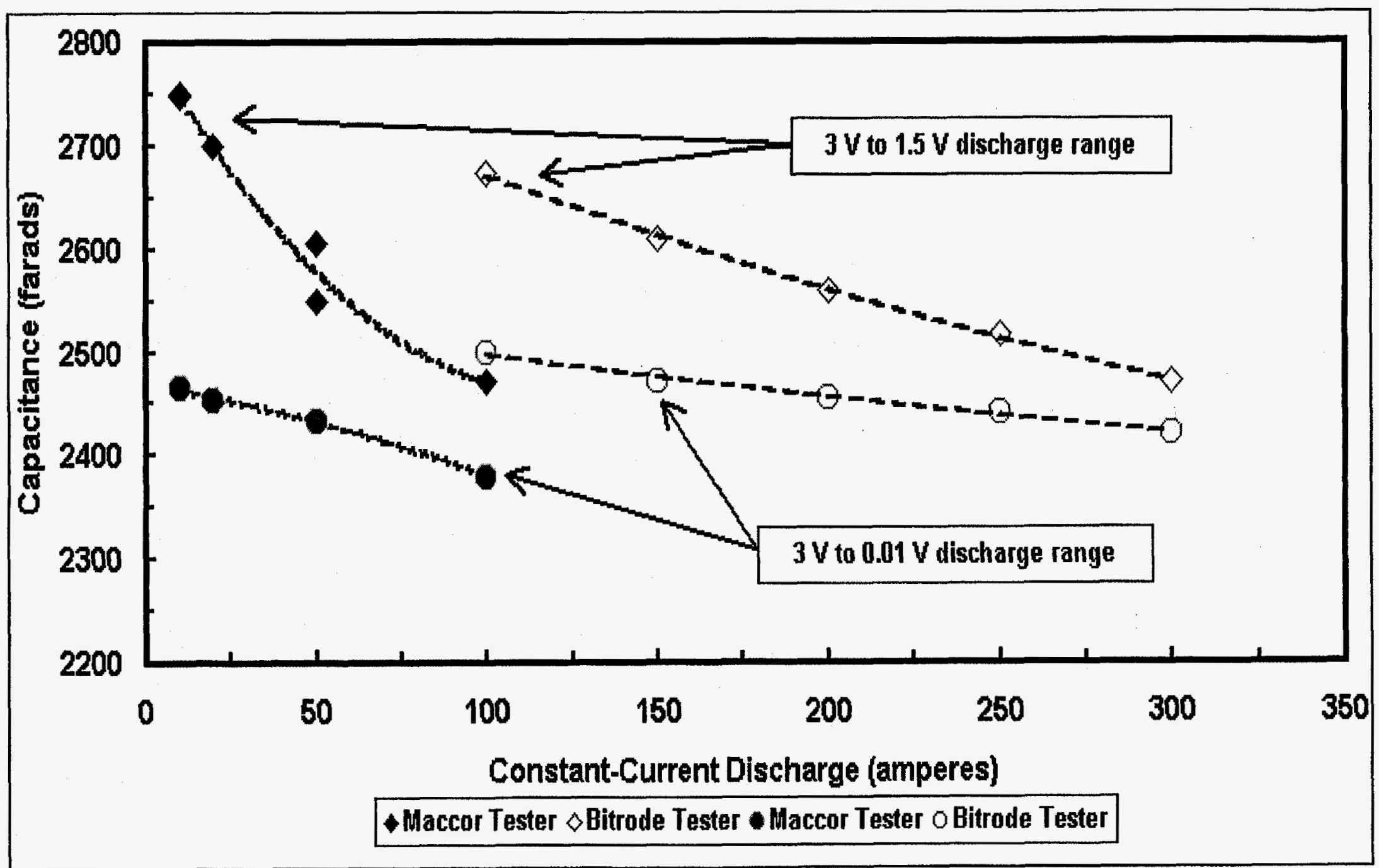

Figure A-37. Measured capacitance for capacitor $\# 1023$ at $23^{\circ} \mathrm{C}$. 


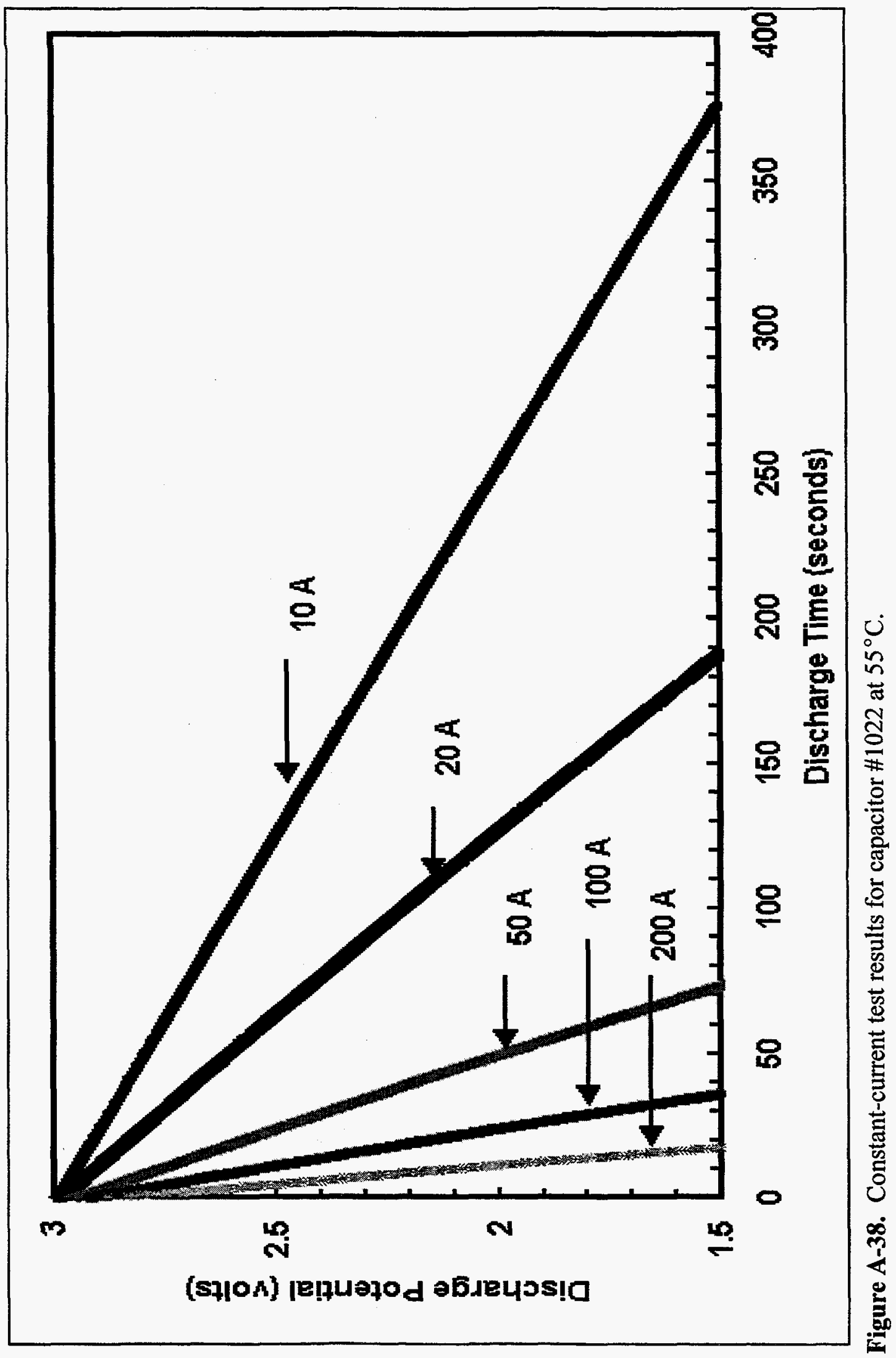




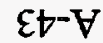

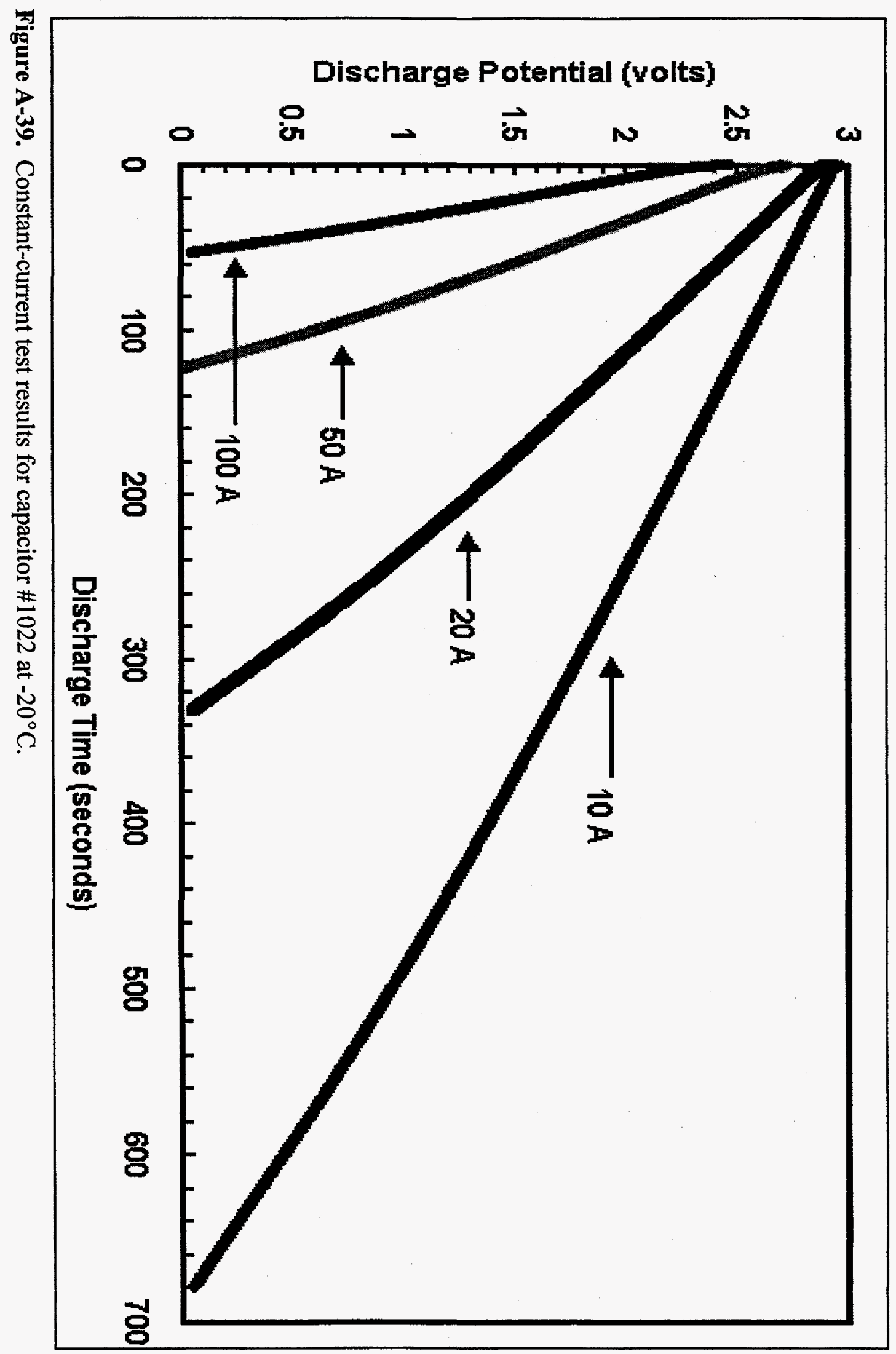




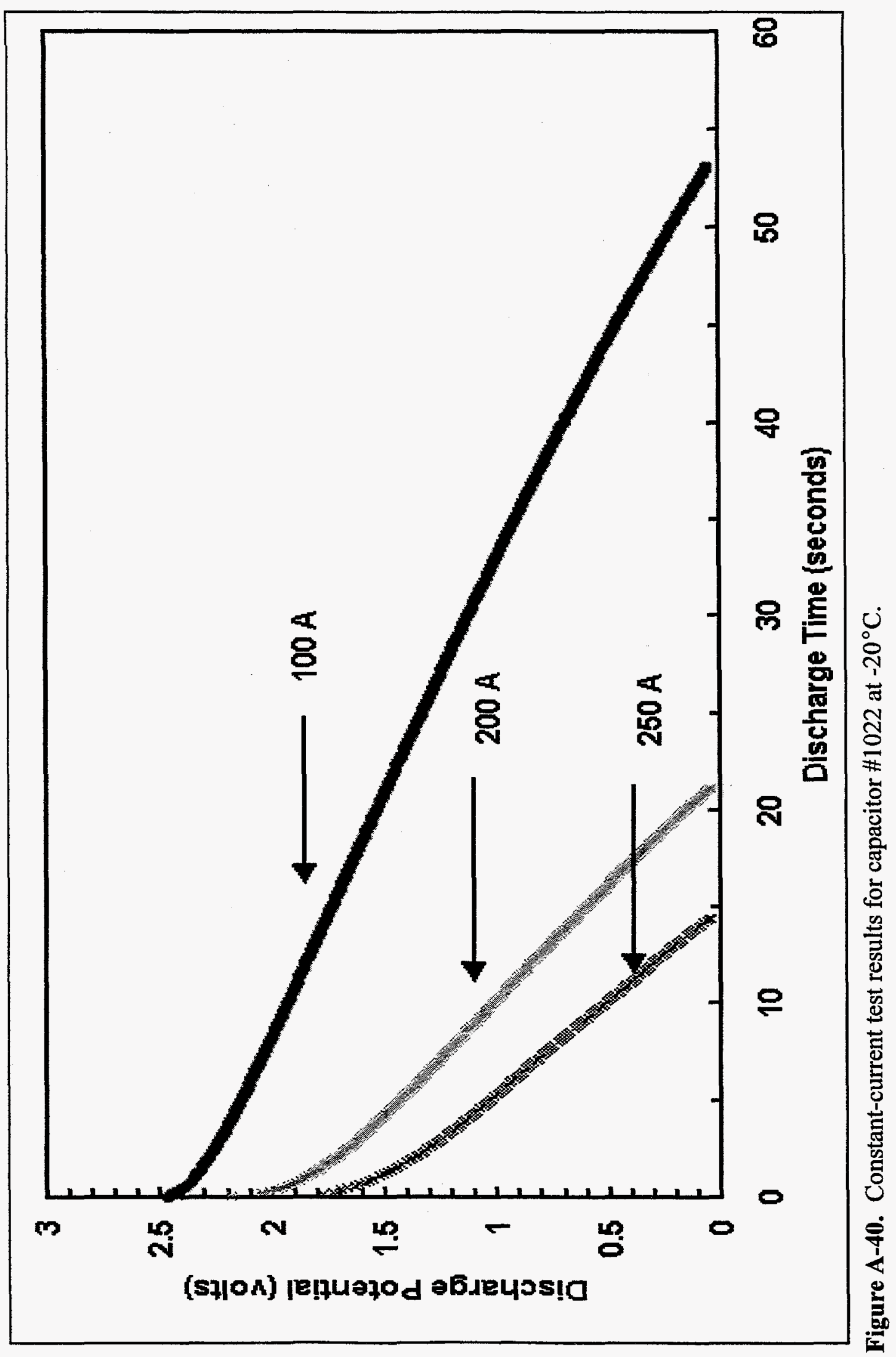




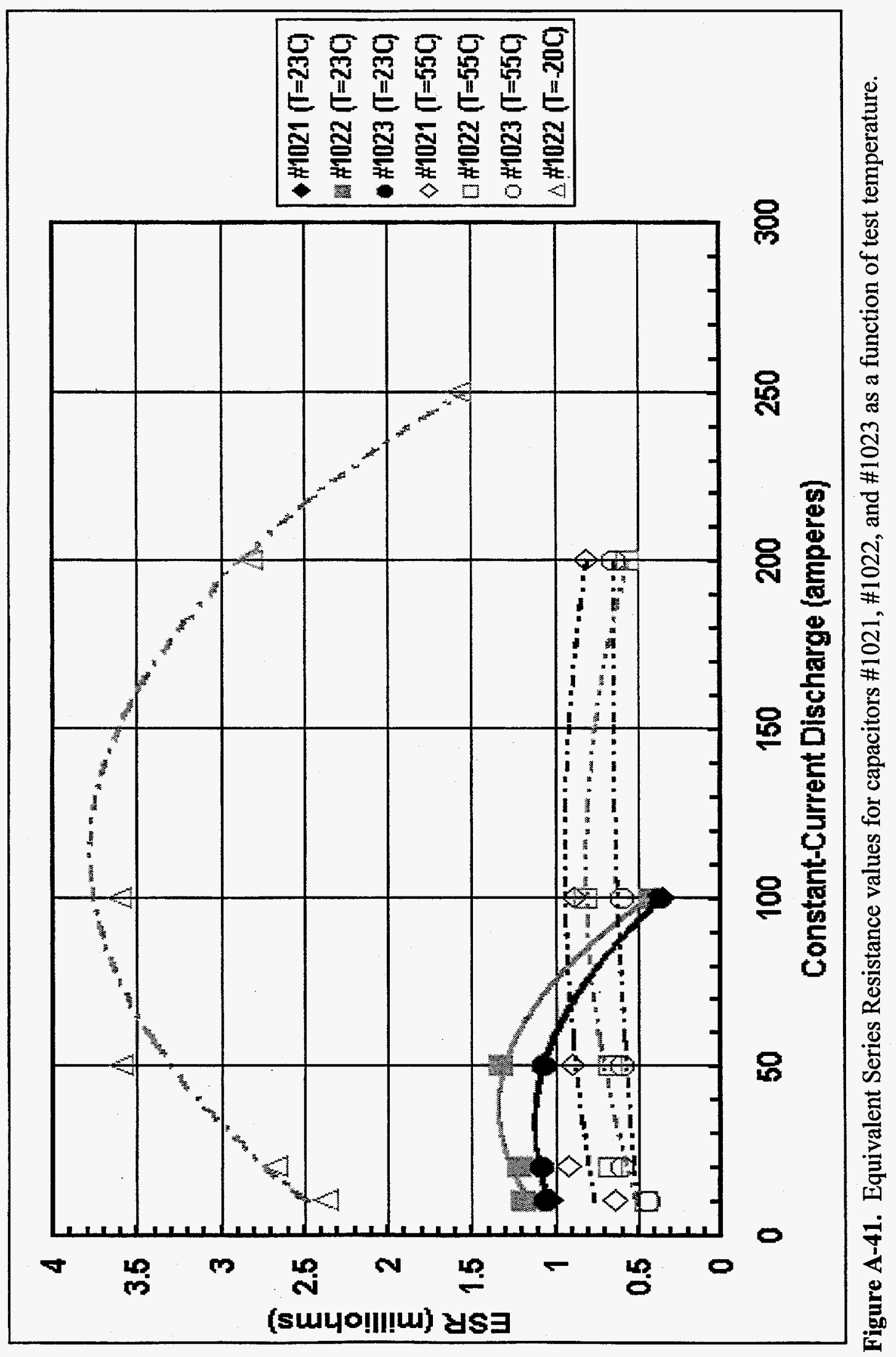




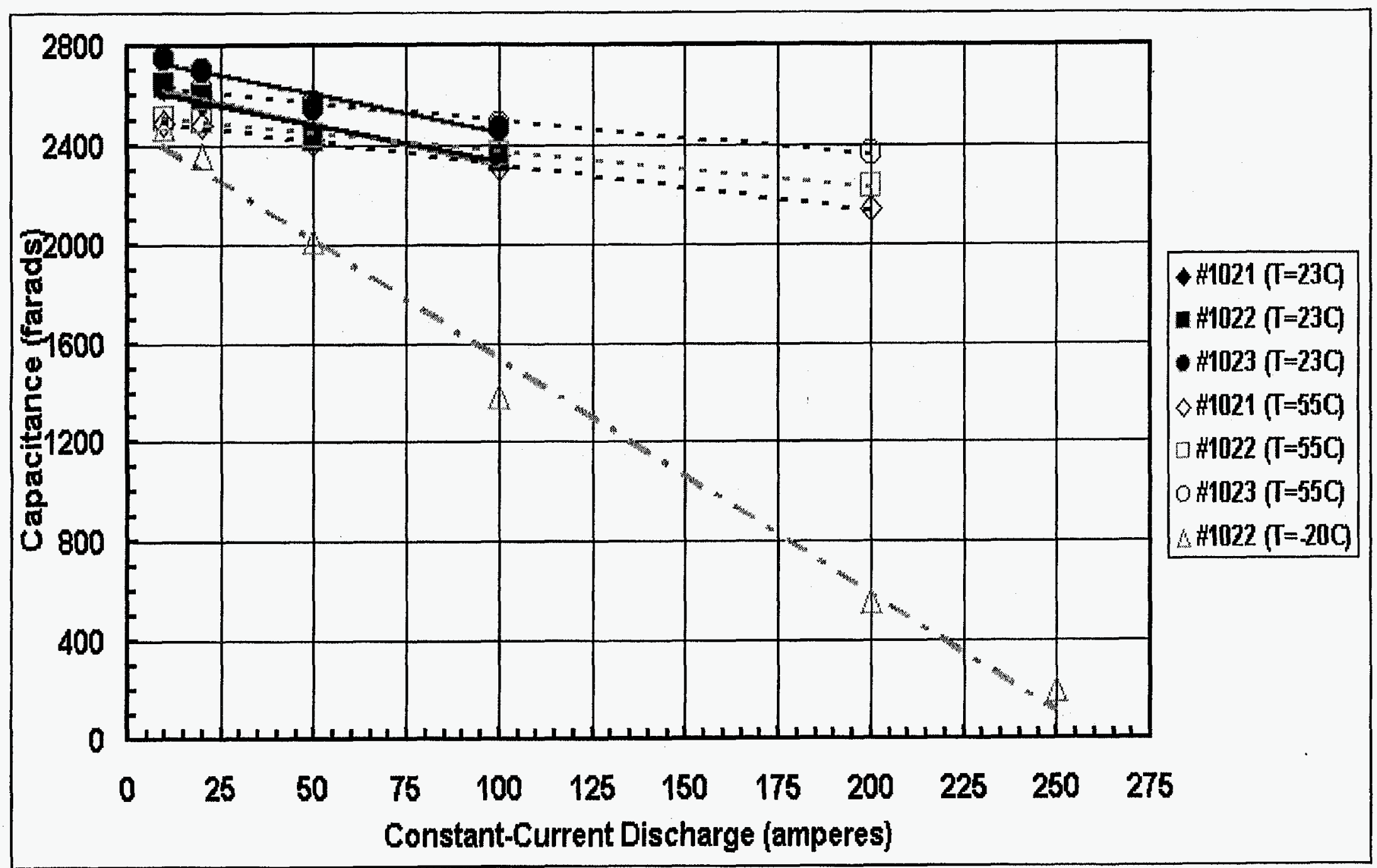

Figure A-42. Capacitance values for capacitors \#1021, \#1022, and \#1023 as a function of constant-current discharge current for test temperatures of $-20^{\circ} \mathrm{C}, 23^{\circ} \mathrm{C}$, and $55^{\circ} \mathrm{C}$. All of these values were determined from data acquired using a Maccor tester. 


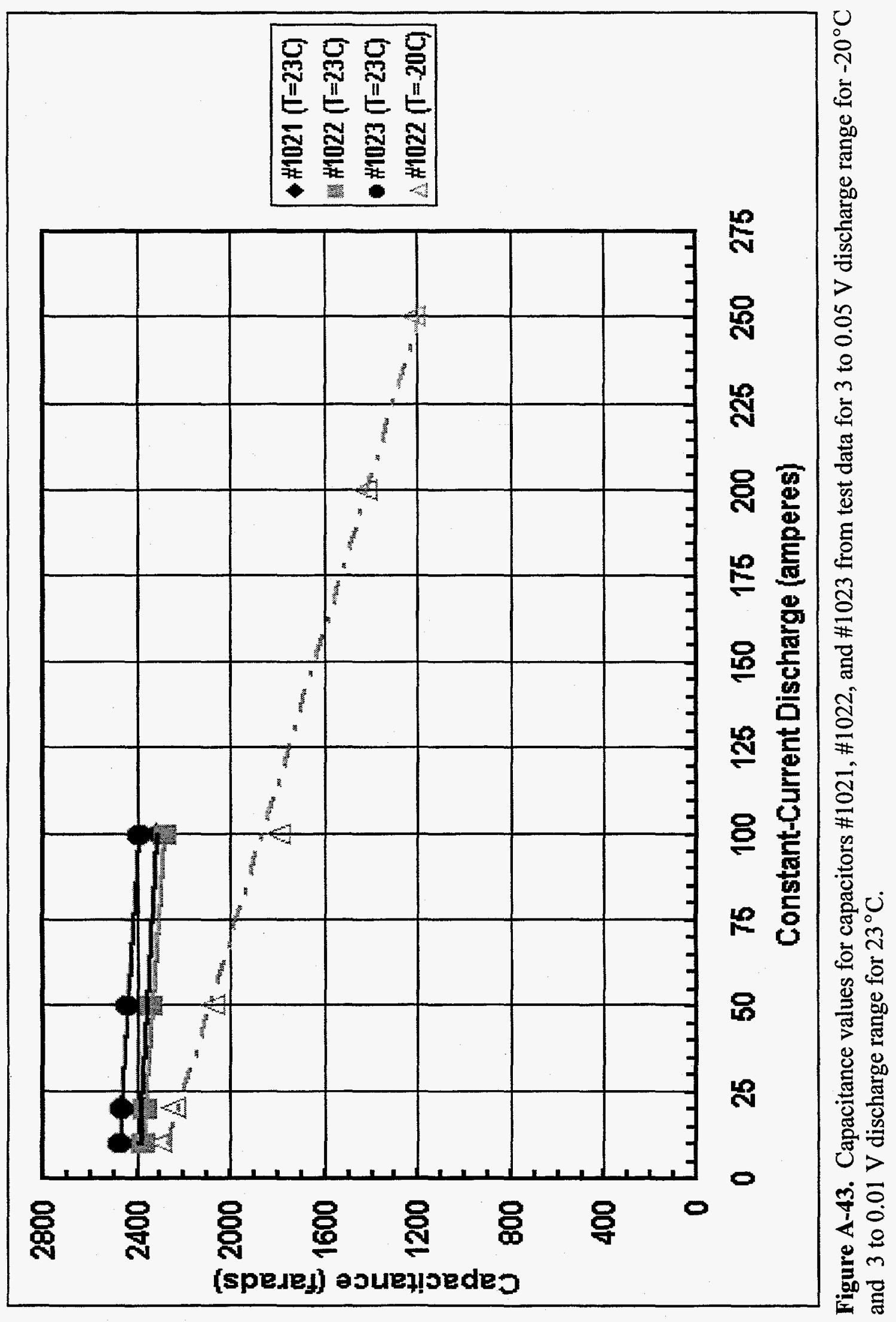




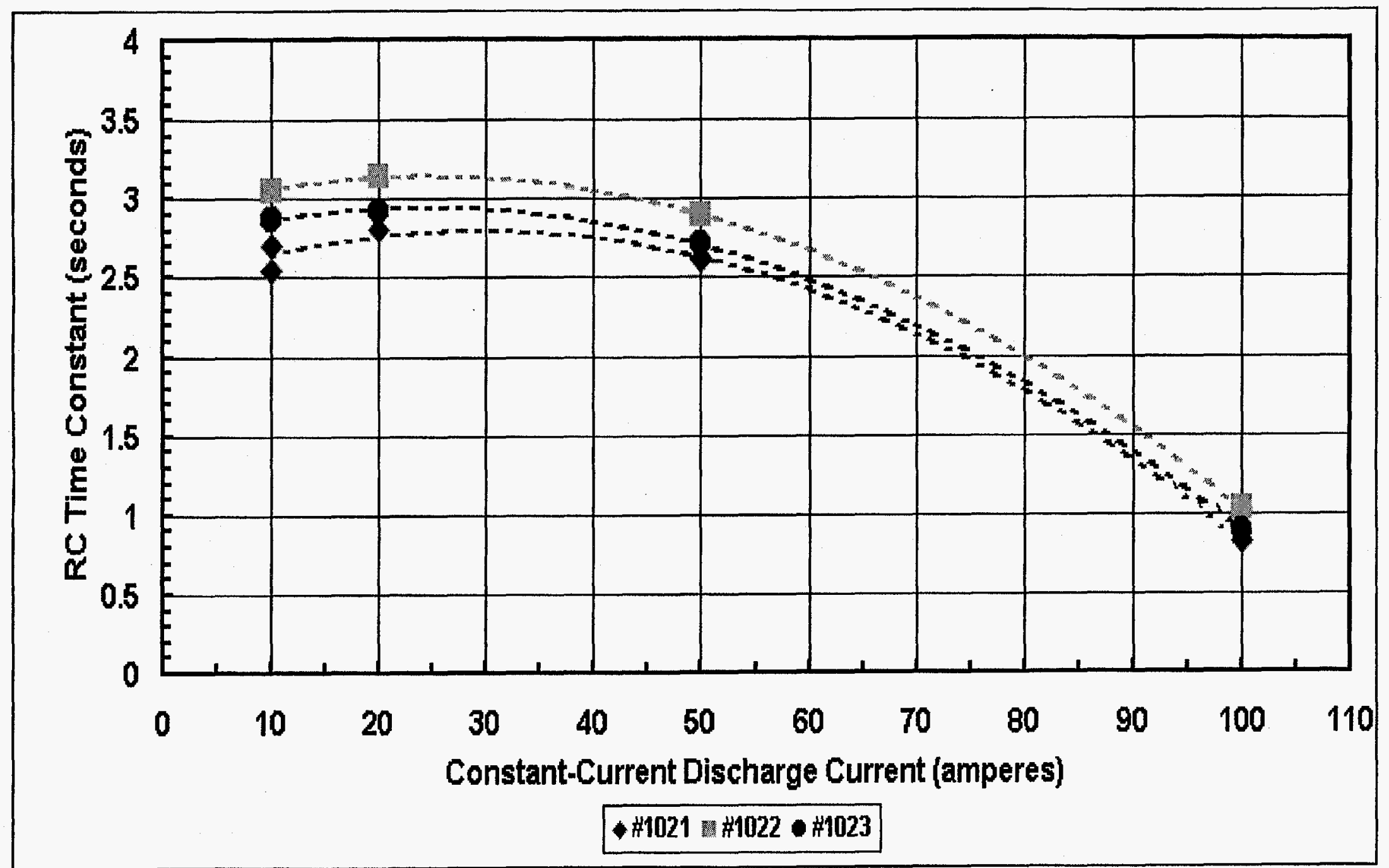

Figure A-44. Data from constant-current tests at $23^{\circ} \mathrm{C}$ for a 3 to $1.5 \mathrm{~V}$ discharge range for capacitors $\# 1021$, \#1022, and \#1023. 


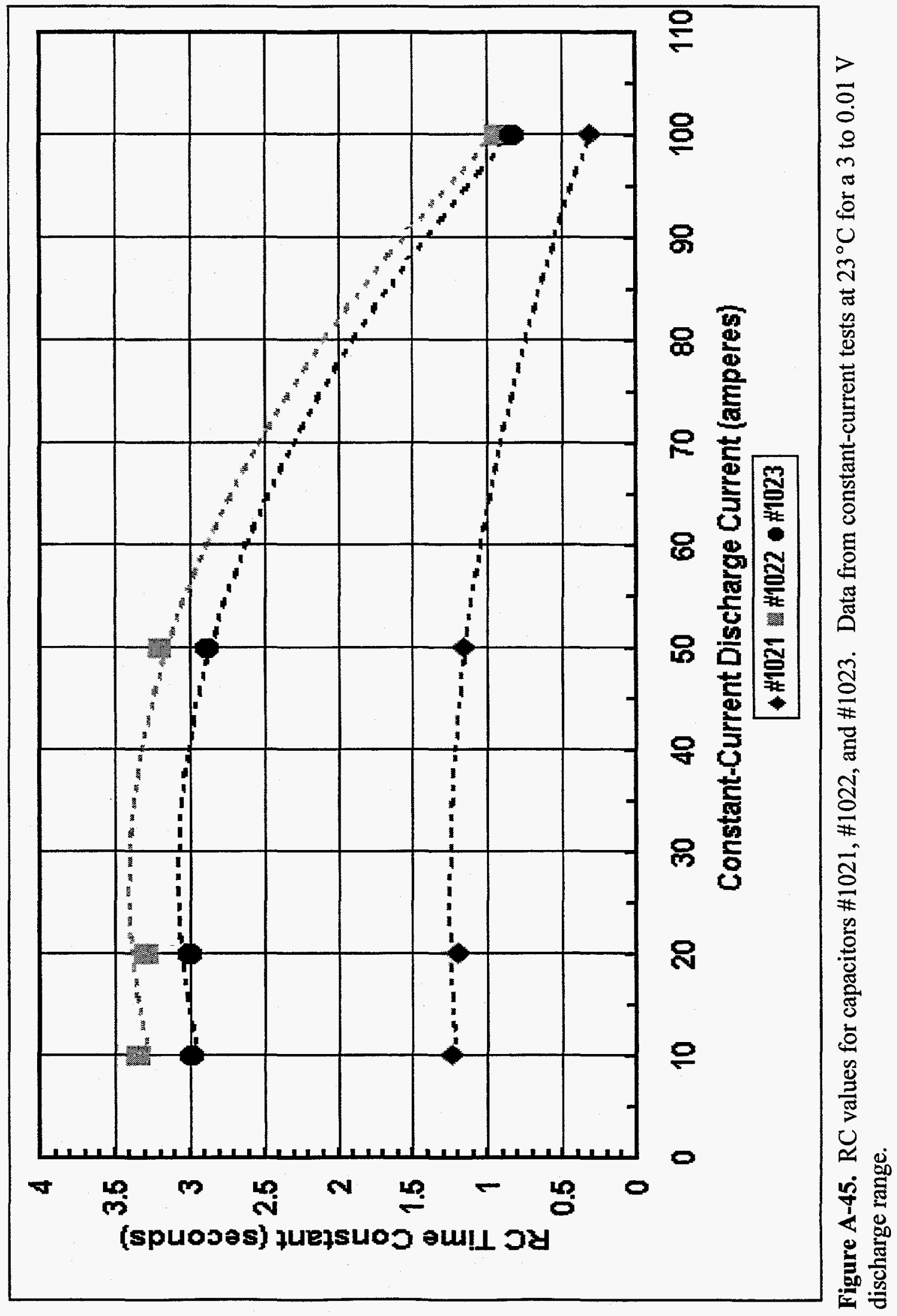




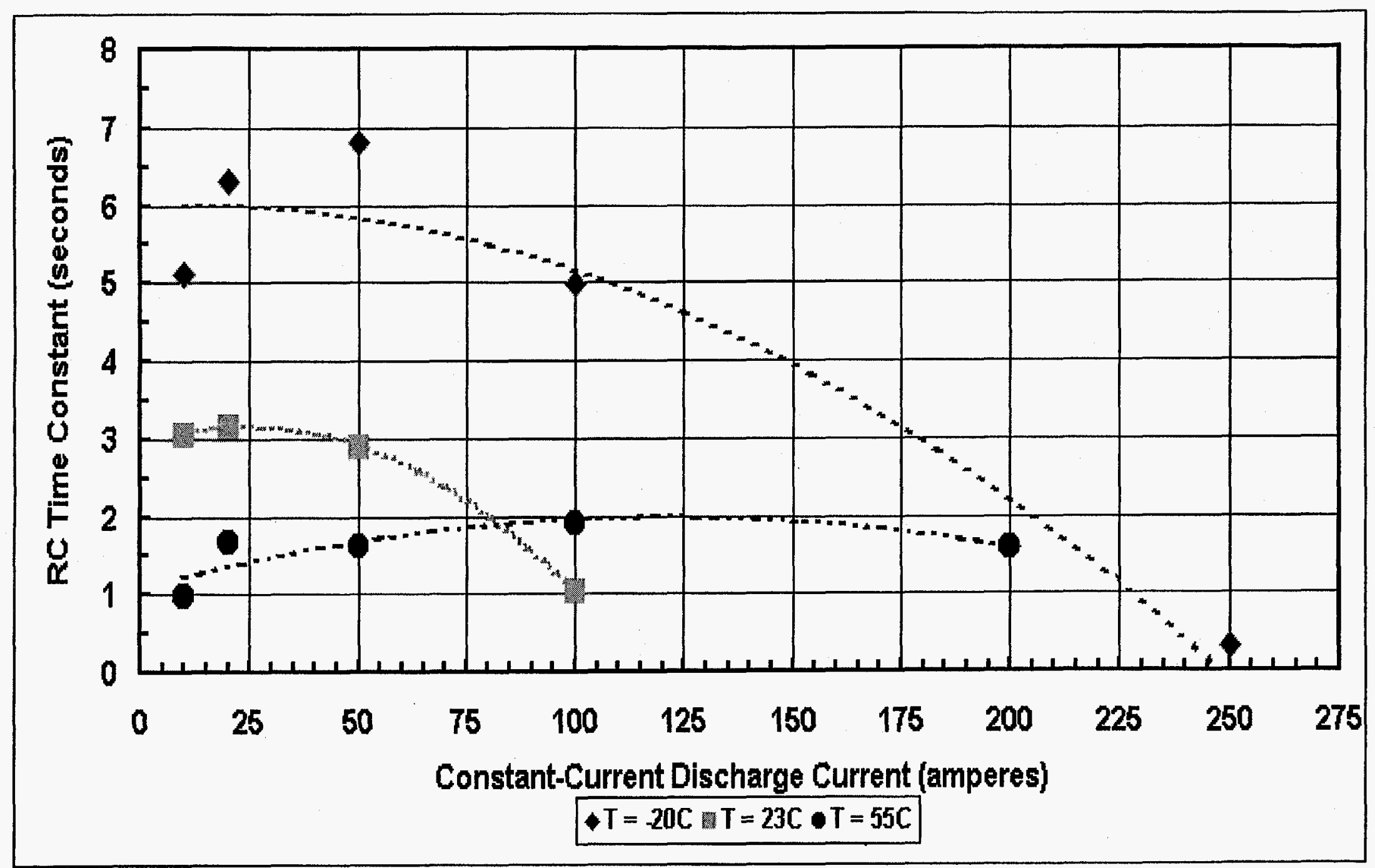

Figure A-46. RC values from constant-current tests for capacitor \#1022 for a discharge range of 3 to $1.5 \mathrm{~V}$. 


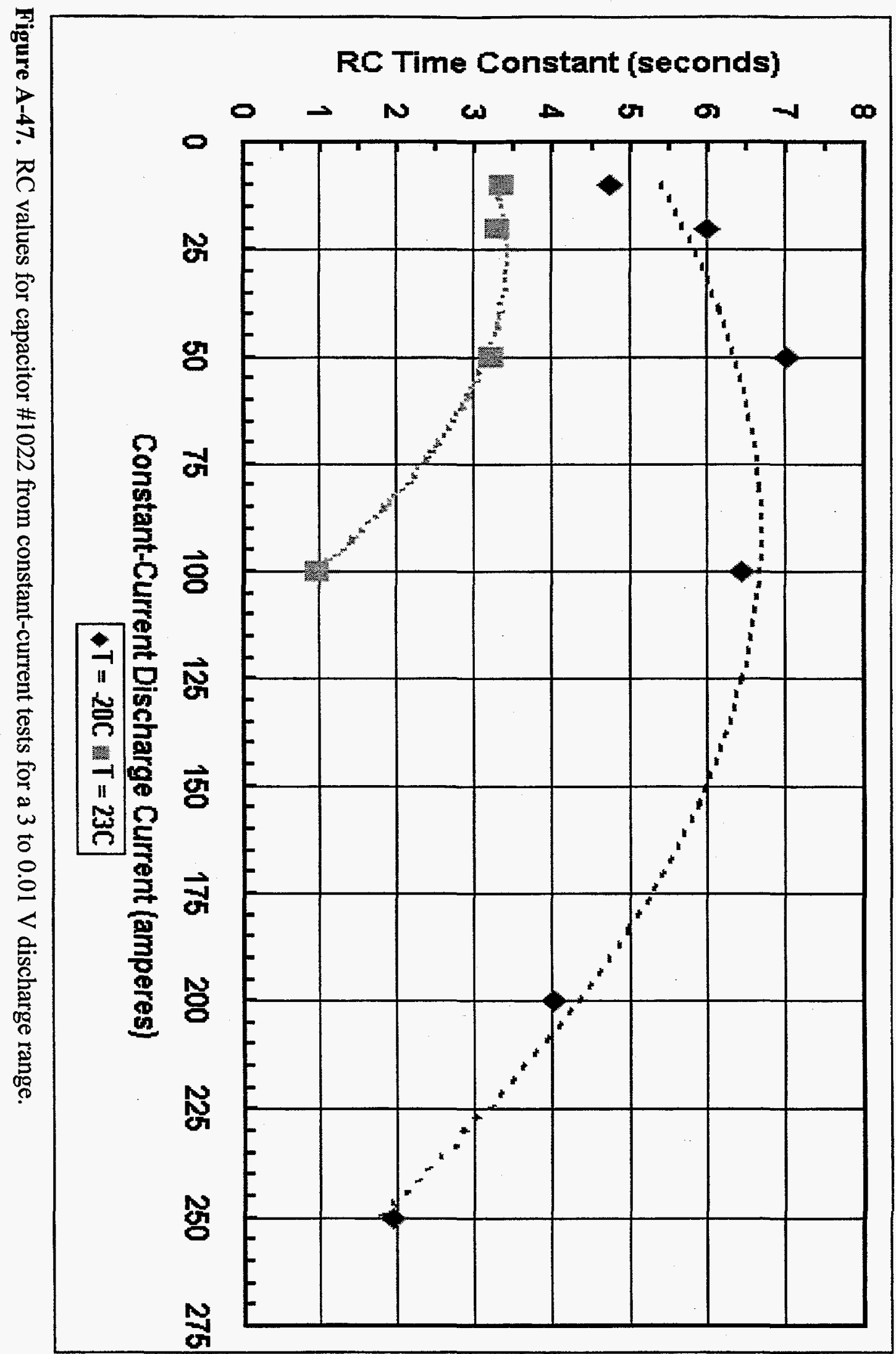




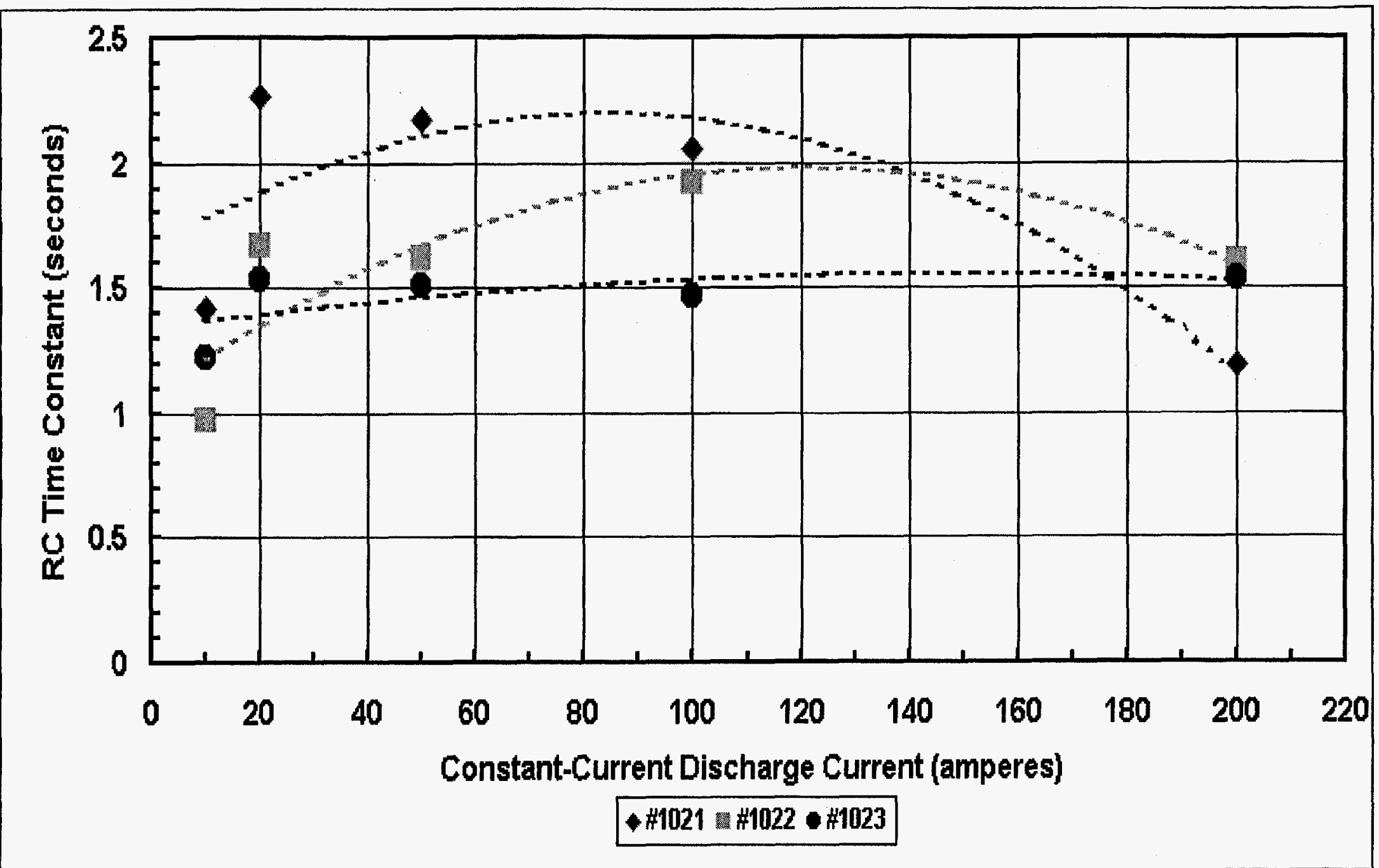

Figure A-48. $\mathrm{RC}$ values for capacitors \#1021, \#1022, and \#1023 from constant-current tests at $55^{\circ} \mathrm{C}$ for a discharge range of 3 to $1.5 \mathrm{~V}$. 


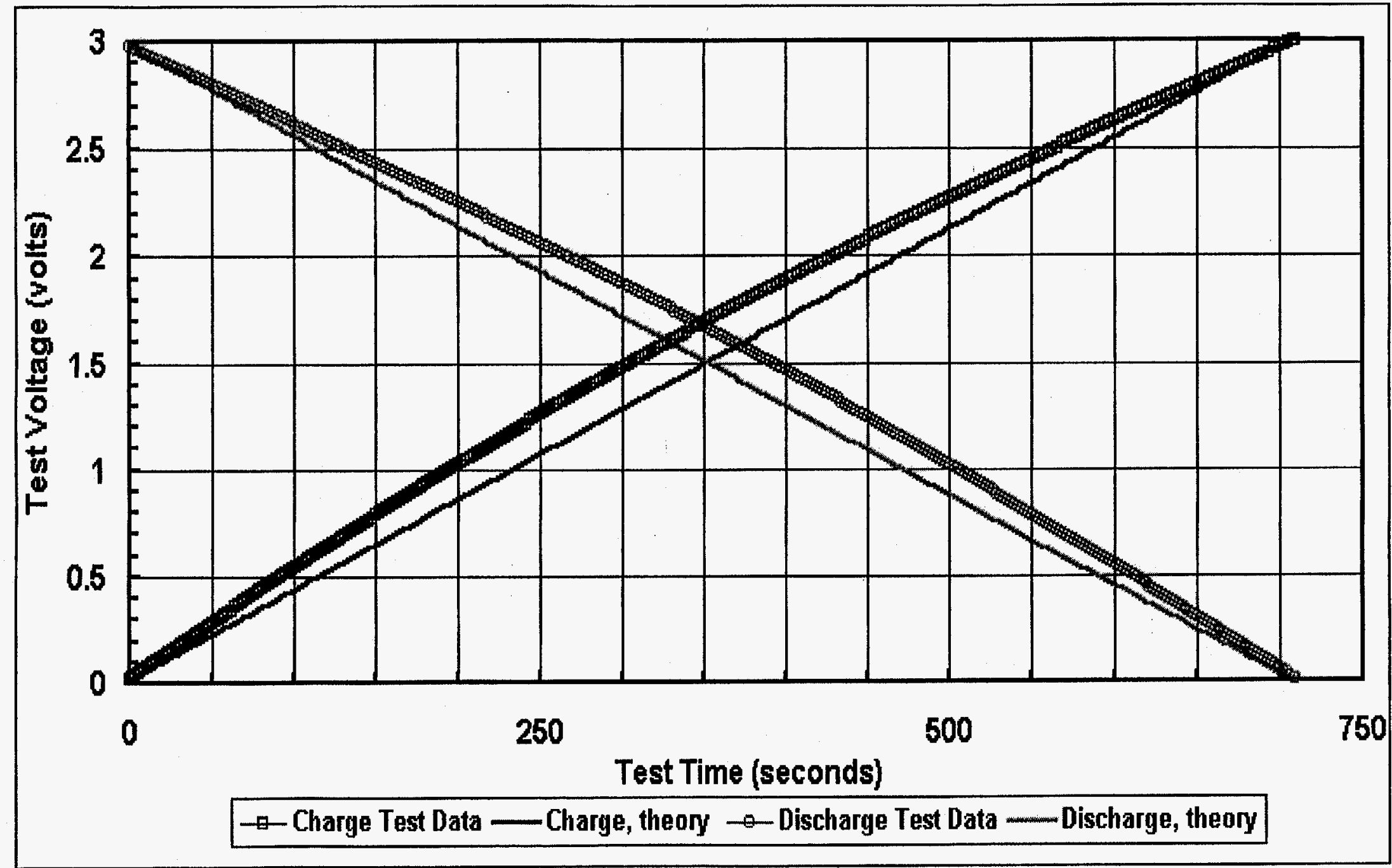

Figure A-49. Test data from $10 \mathrm{~A}$ constant-current charge/discharge test for capacitor $\# 1022$ at $23^{\circ} \mathrm{C}$. 
$t \varsigma-\forall$

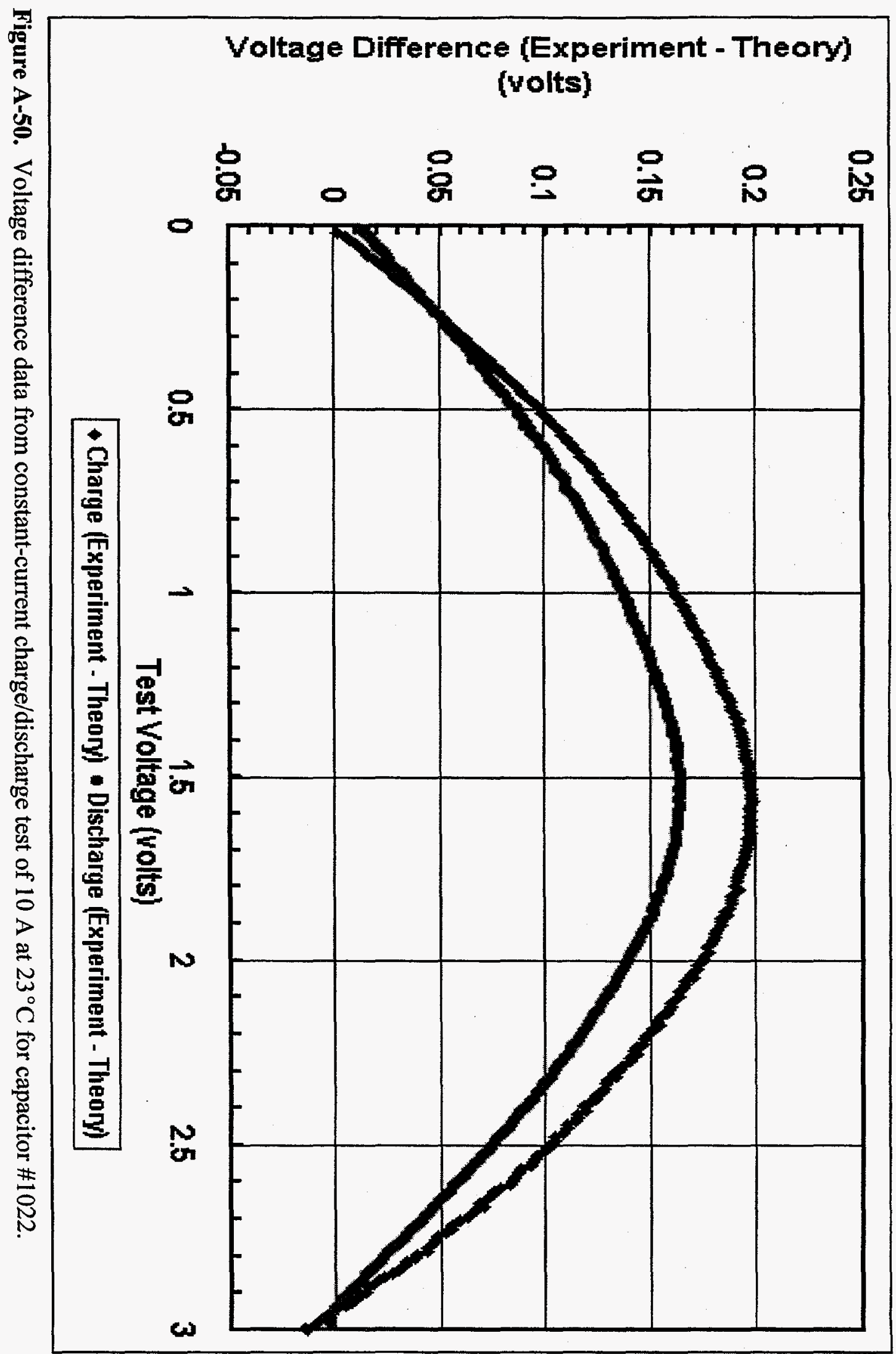




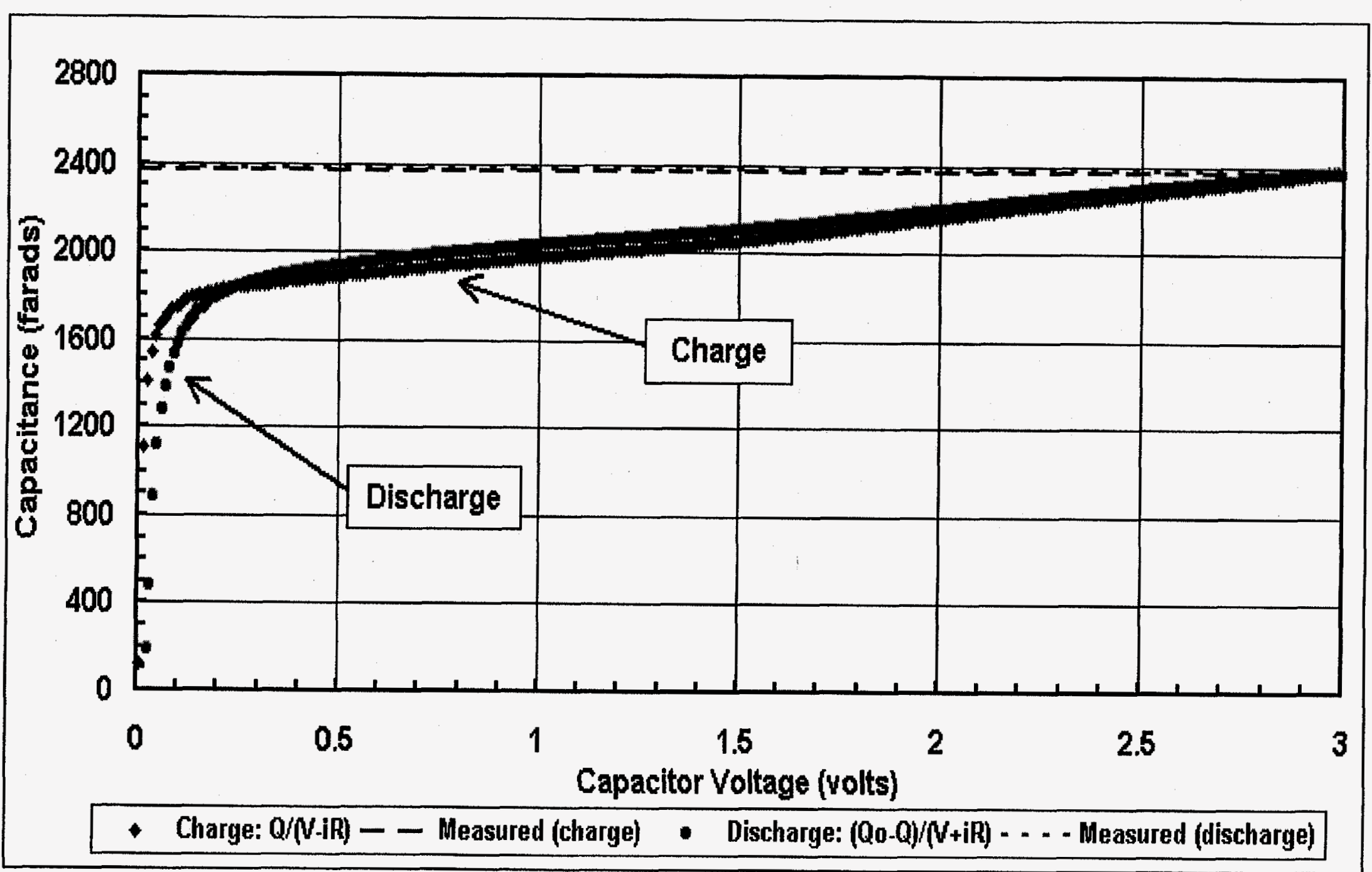

Figure A-51. Calculated capacitance data for capacitor $\# 1022$ from $10 \mathrm{~A}$ constant-current test at $23^{\circ} \mathrm{C}$. 


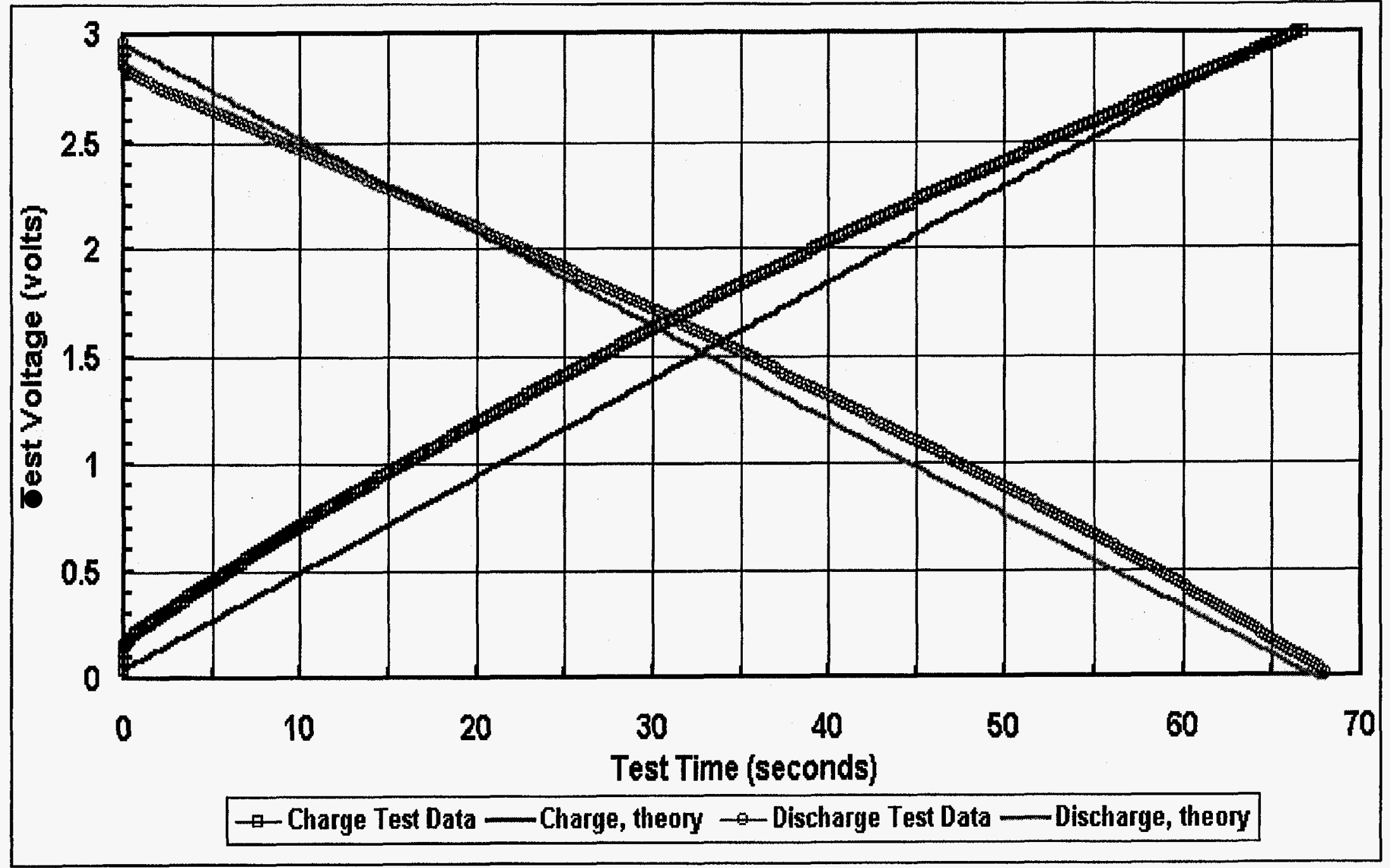

Figure A-52. Data from 100 A constant-current charge/discharge test at $23^{\circ} \mathrm{C}$ for capacitor $\# 1022$. 


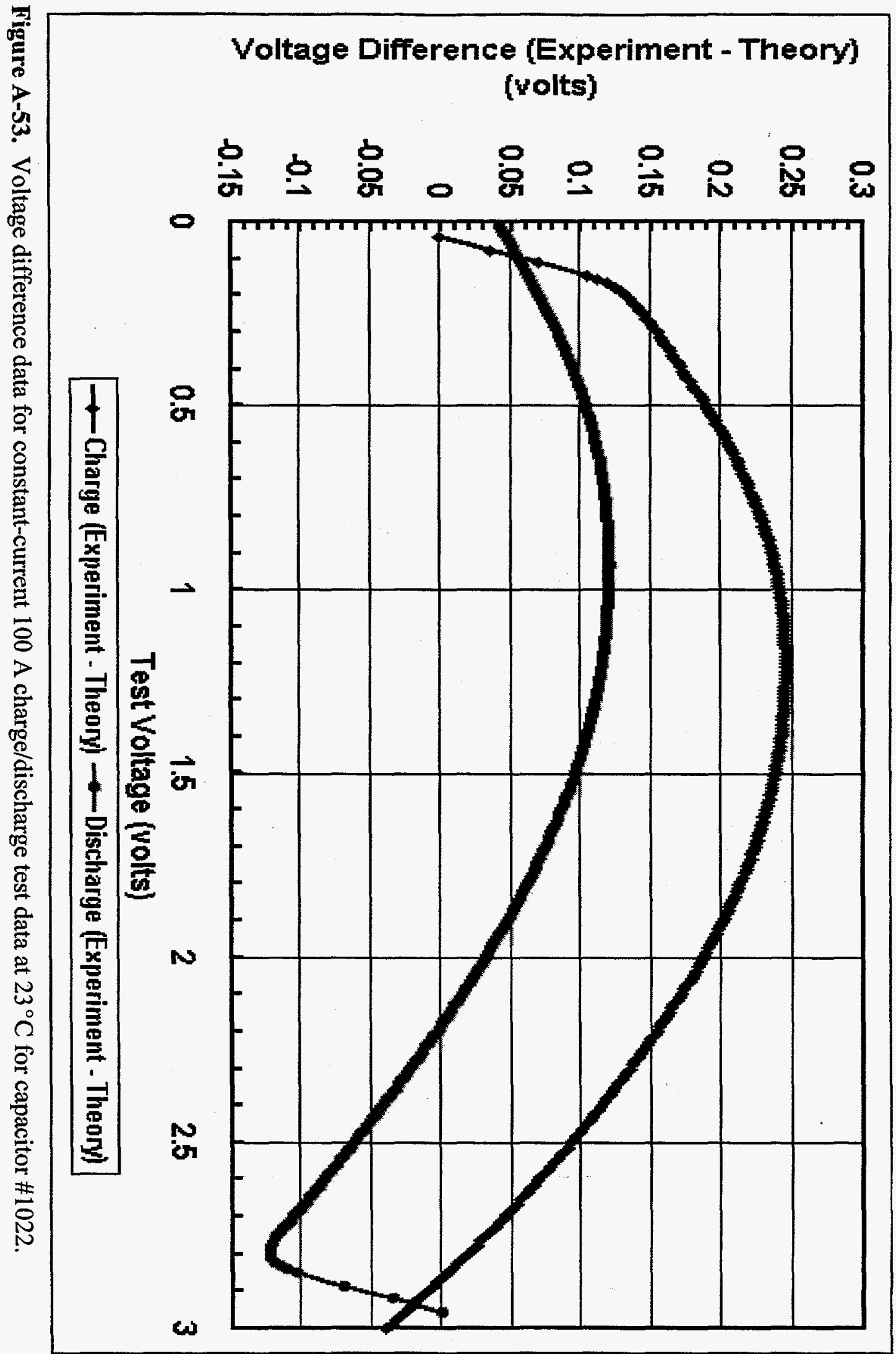




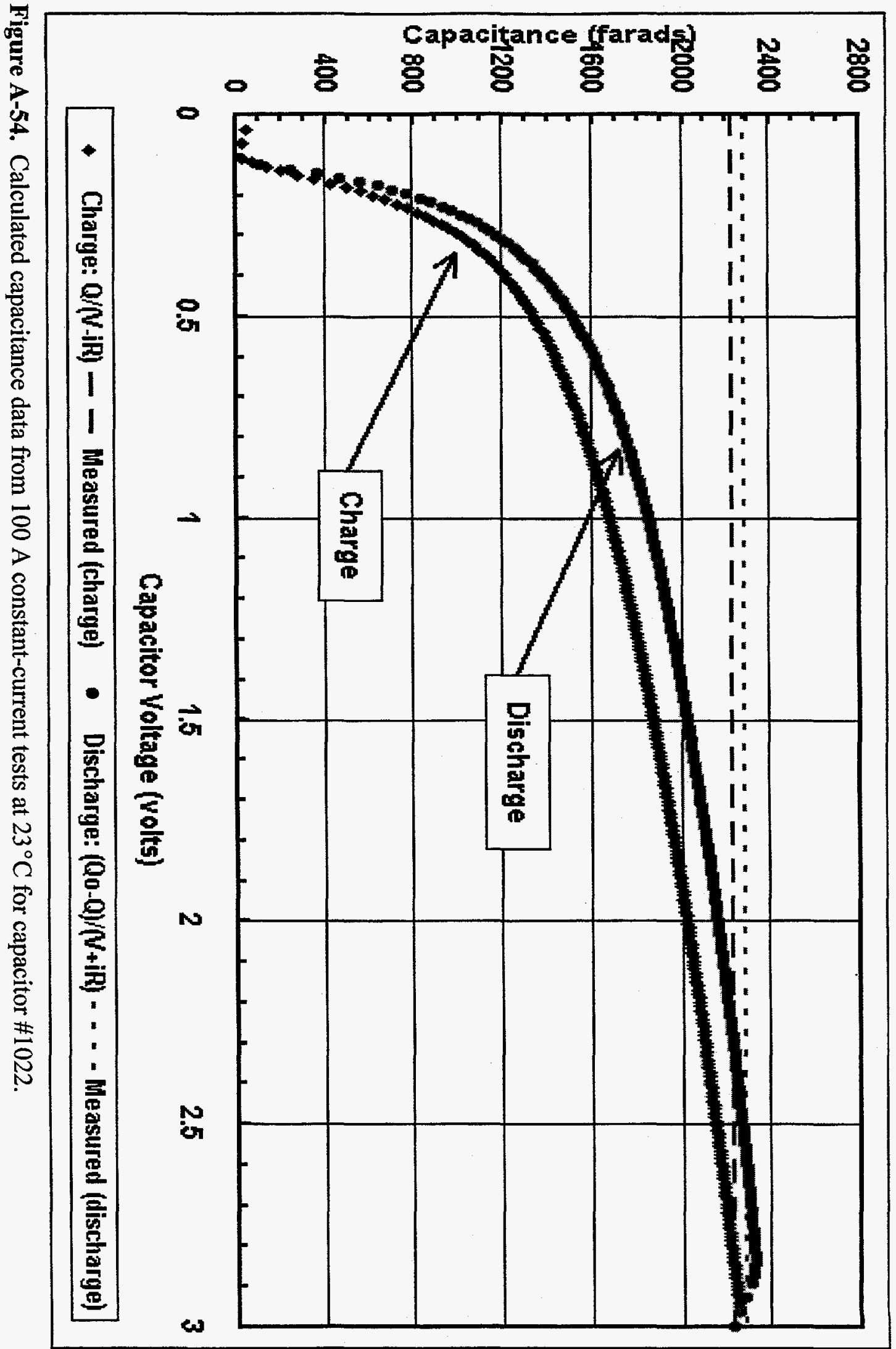




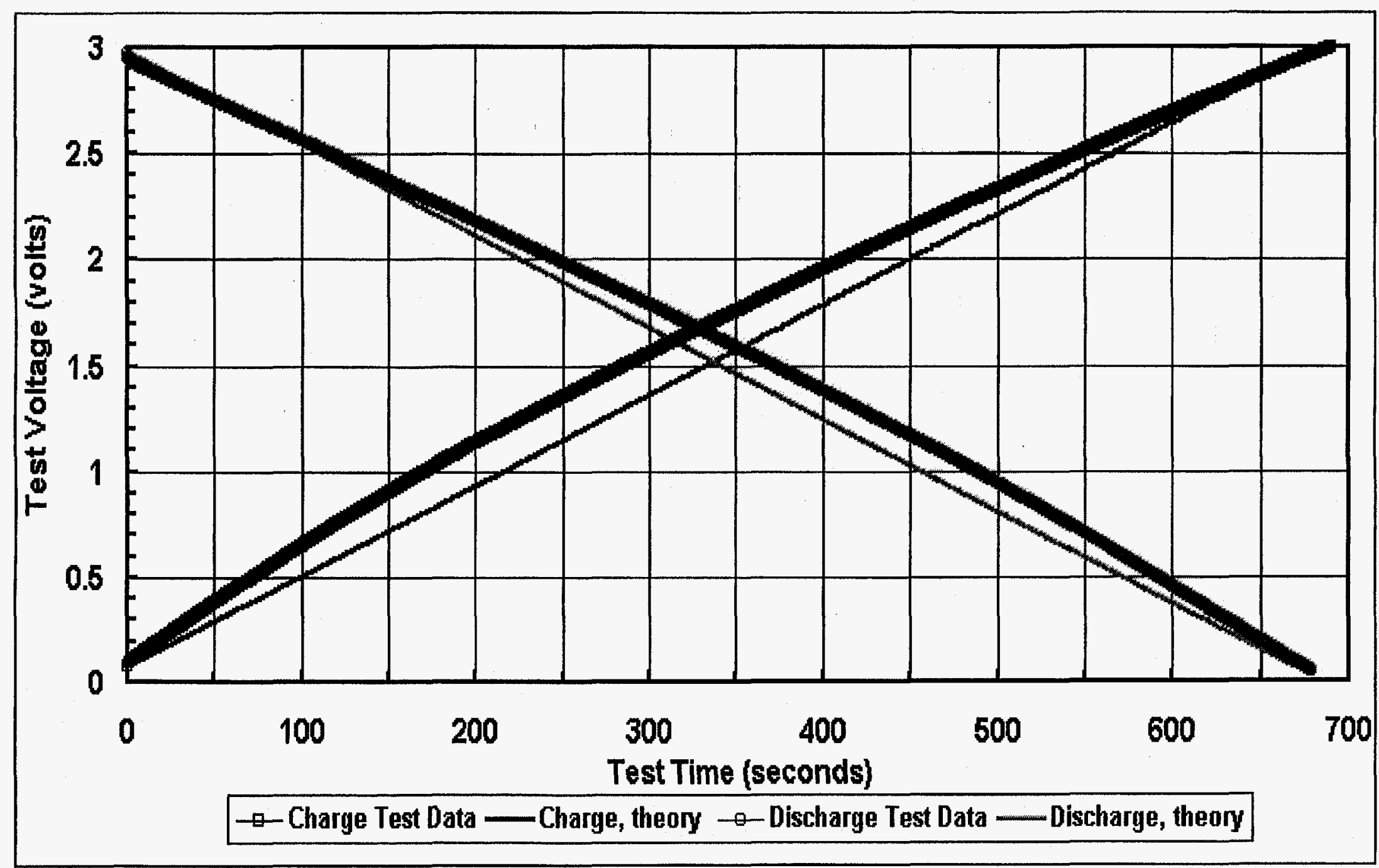

Figure A-55. Data from $10 \mathrm{~A}$ constant-current charge/discharge test at $-20^{\circ} \mathrm{C}$ for capacitor $\# 1022$. 


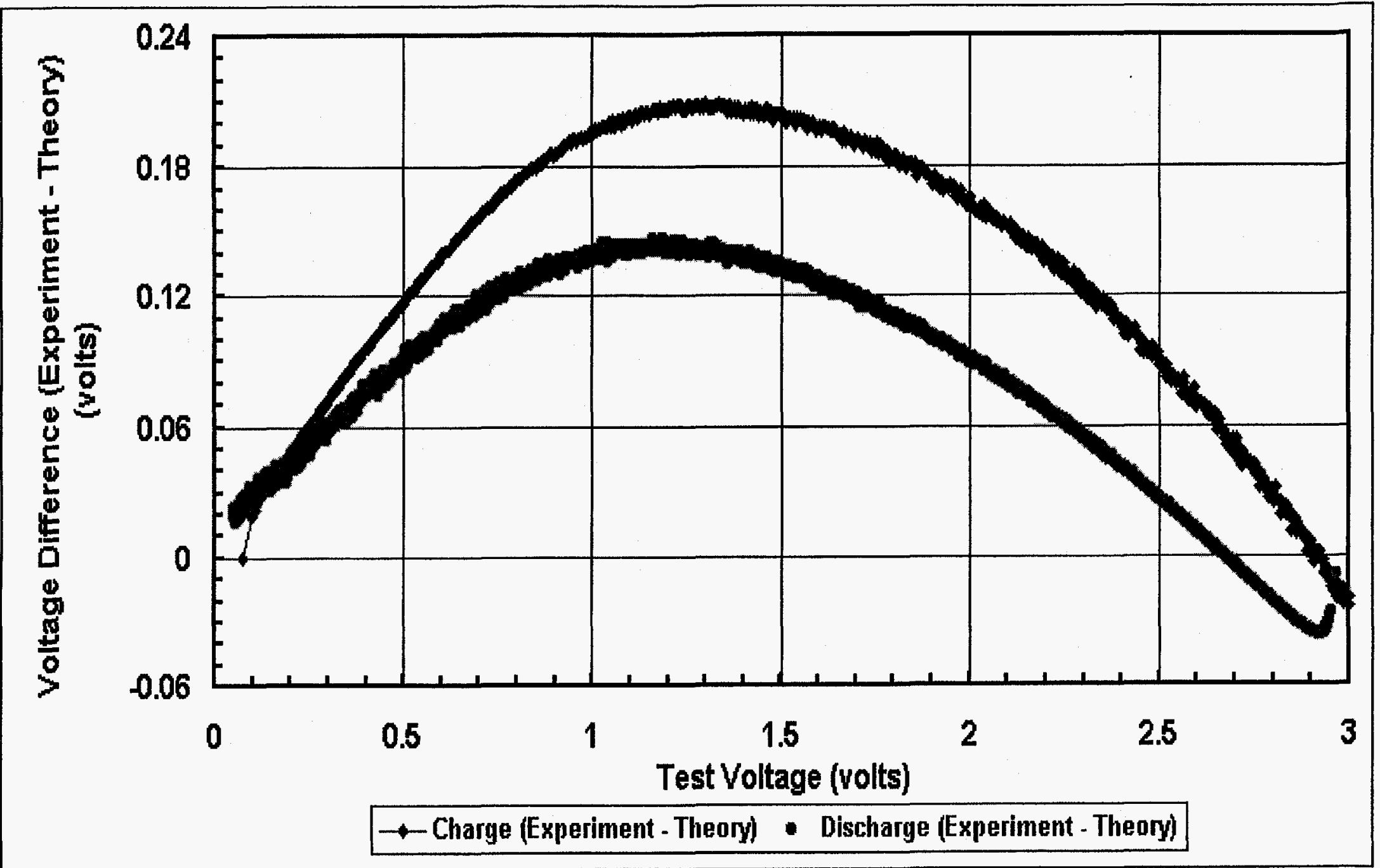

Figure A-56. Voltage difference data from $10 \mathrm{~A}$ constant-current charge/discharge at $-20^{\circ} \mathrm{C}$ for capacitor $\# 1022$. 


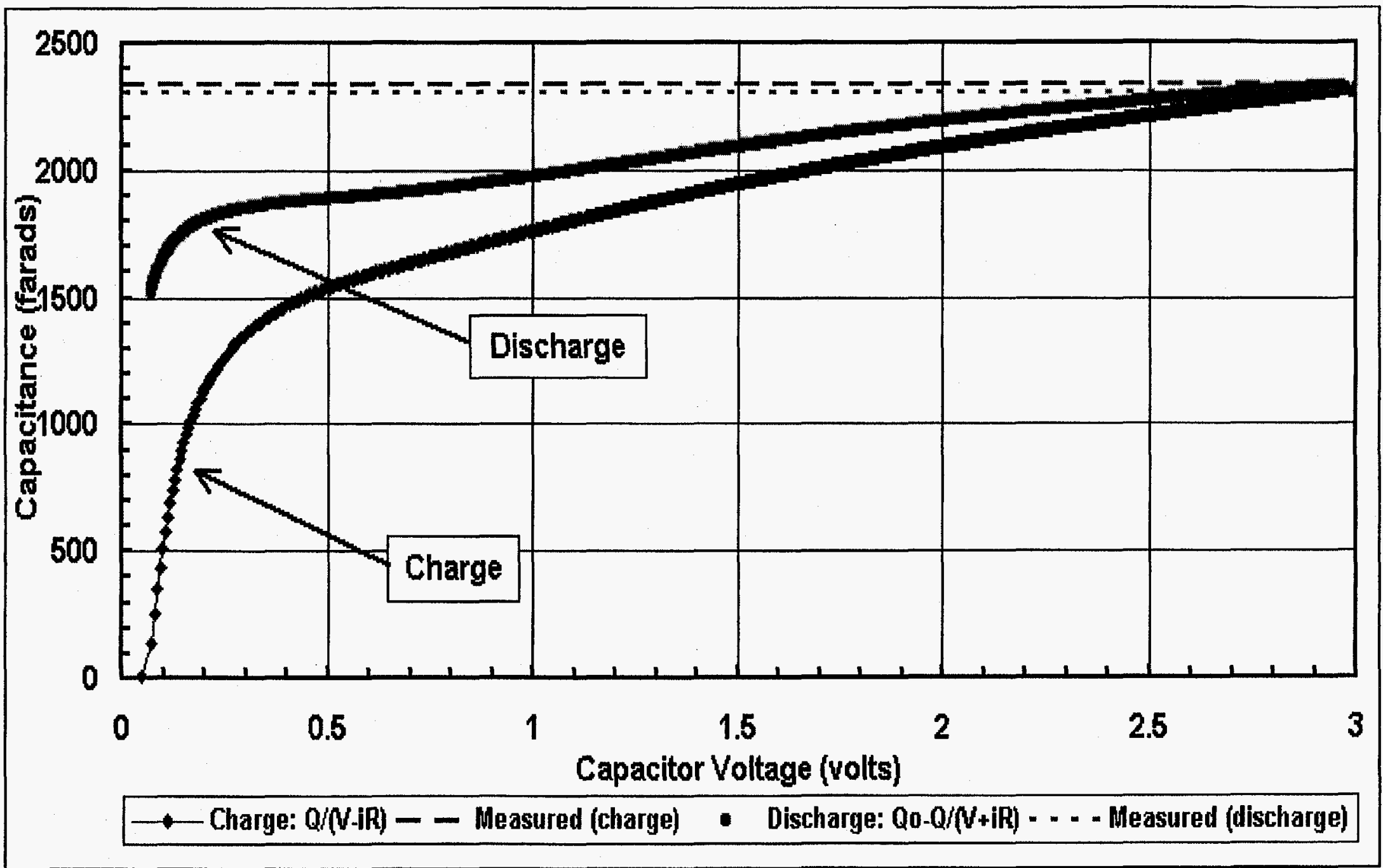

Figure A-57. Calculated capacitance for capacitor $\# 1022$ from 10 A constant-current test at $-20^{\circ} \mathrm{C}$. 


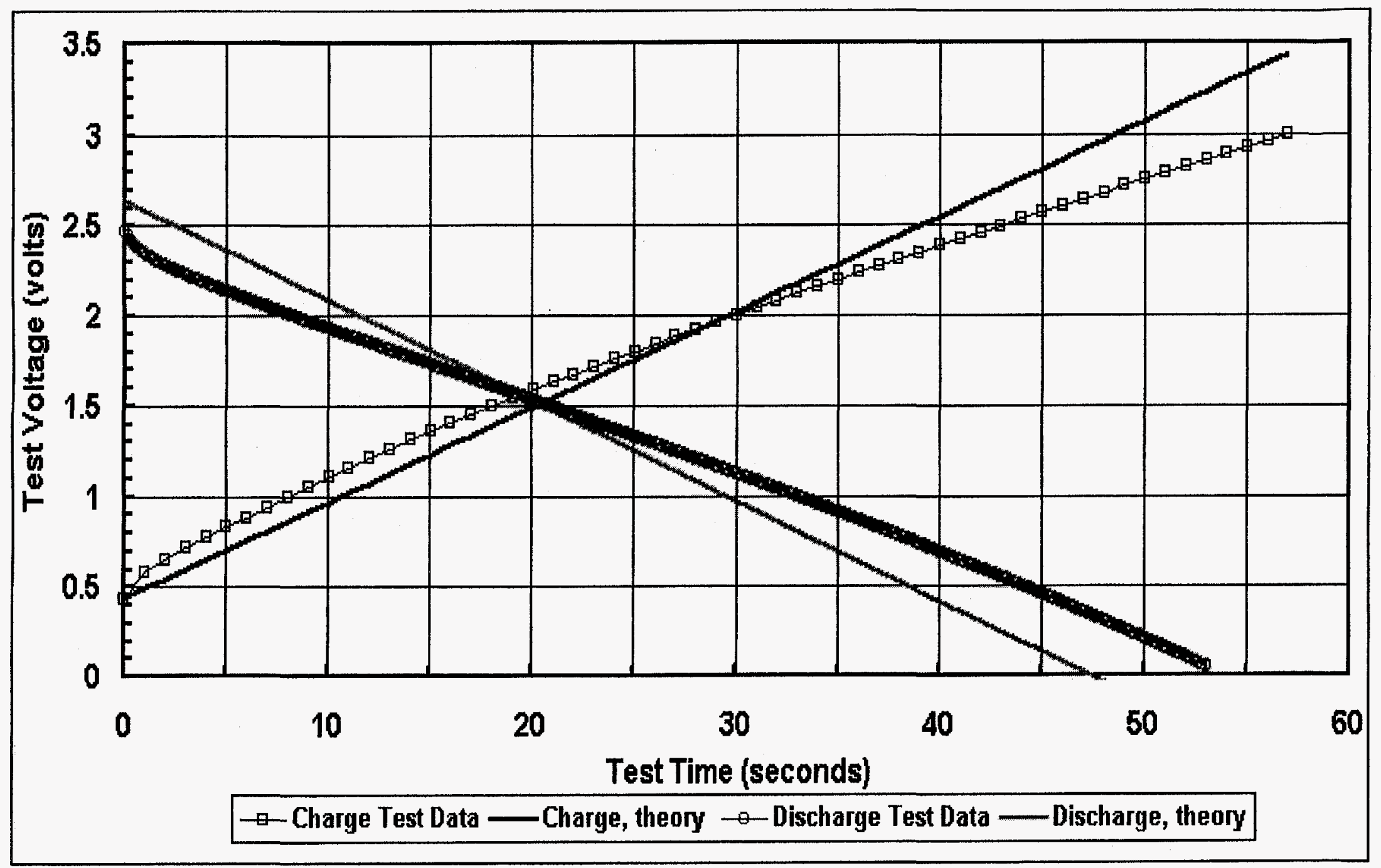

Figure A-58. $100 \mathrm{~A}$ constant-current charge/discharge test data at $-20^{\circ} \mathrm{C}$ for capacitor $\# 1022$. 


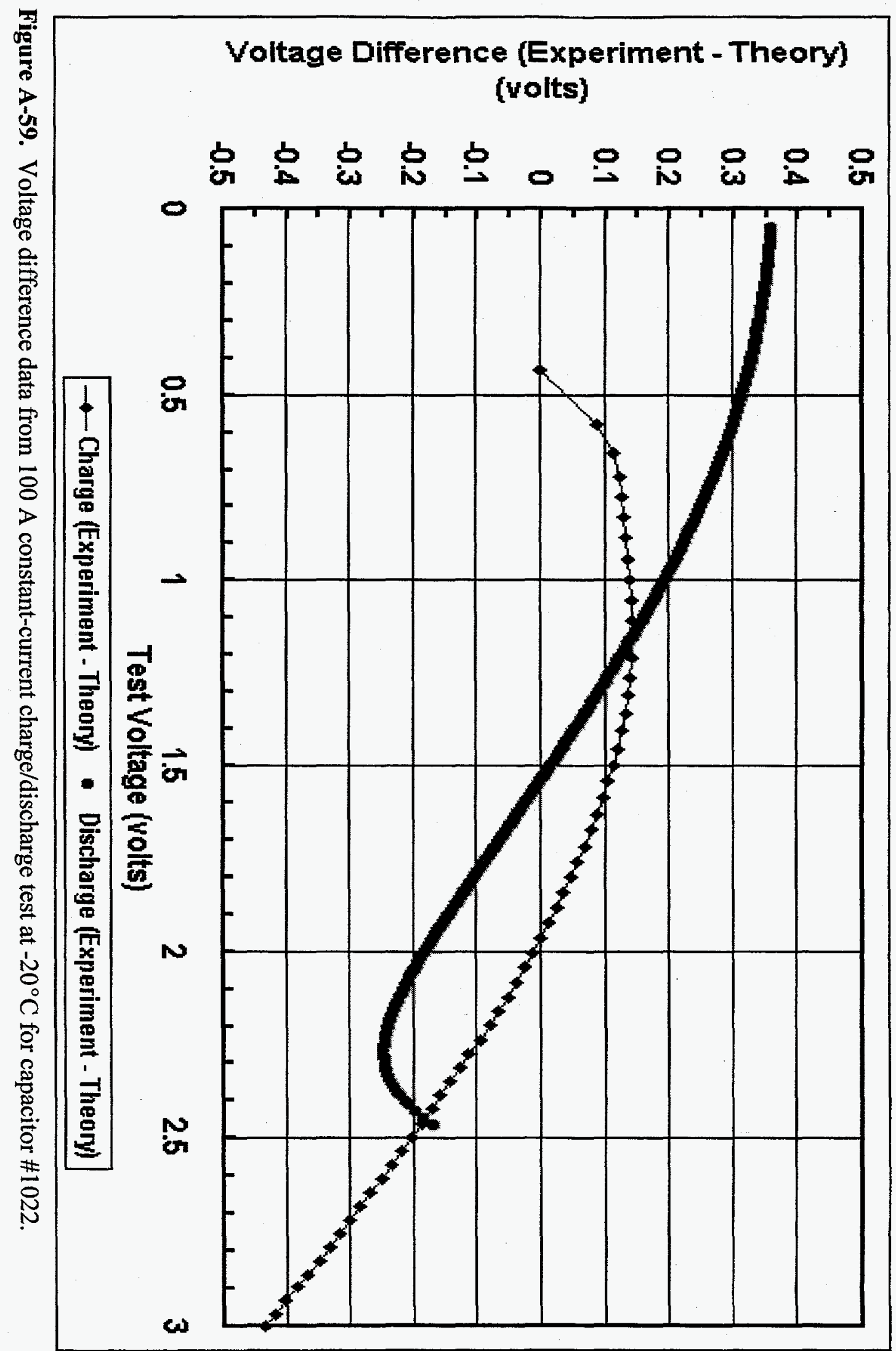




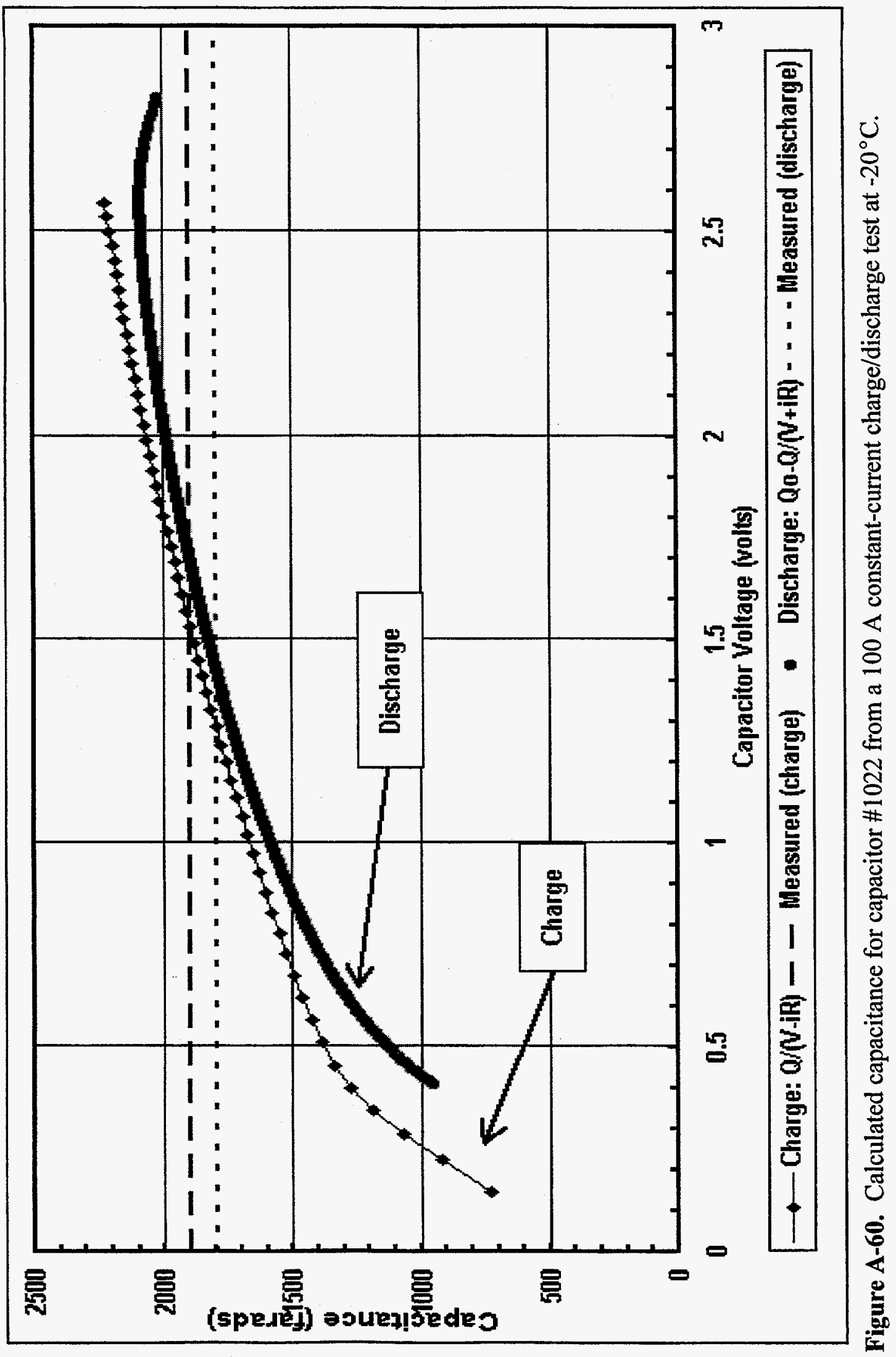




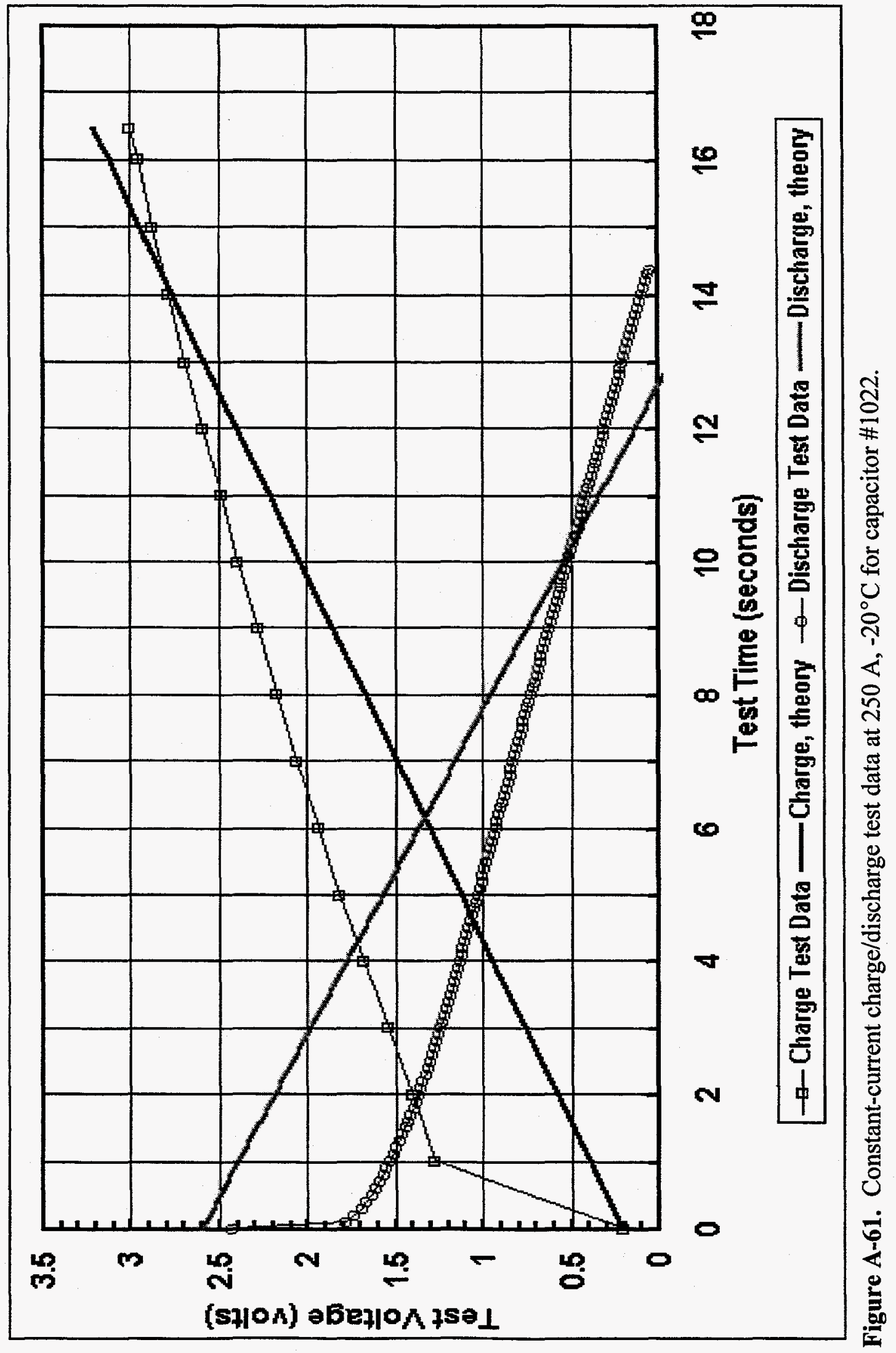




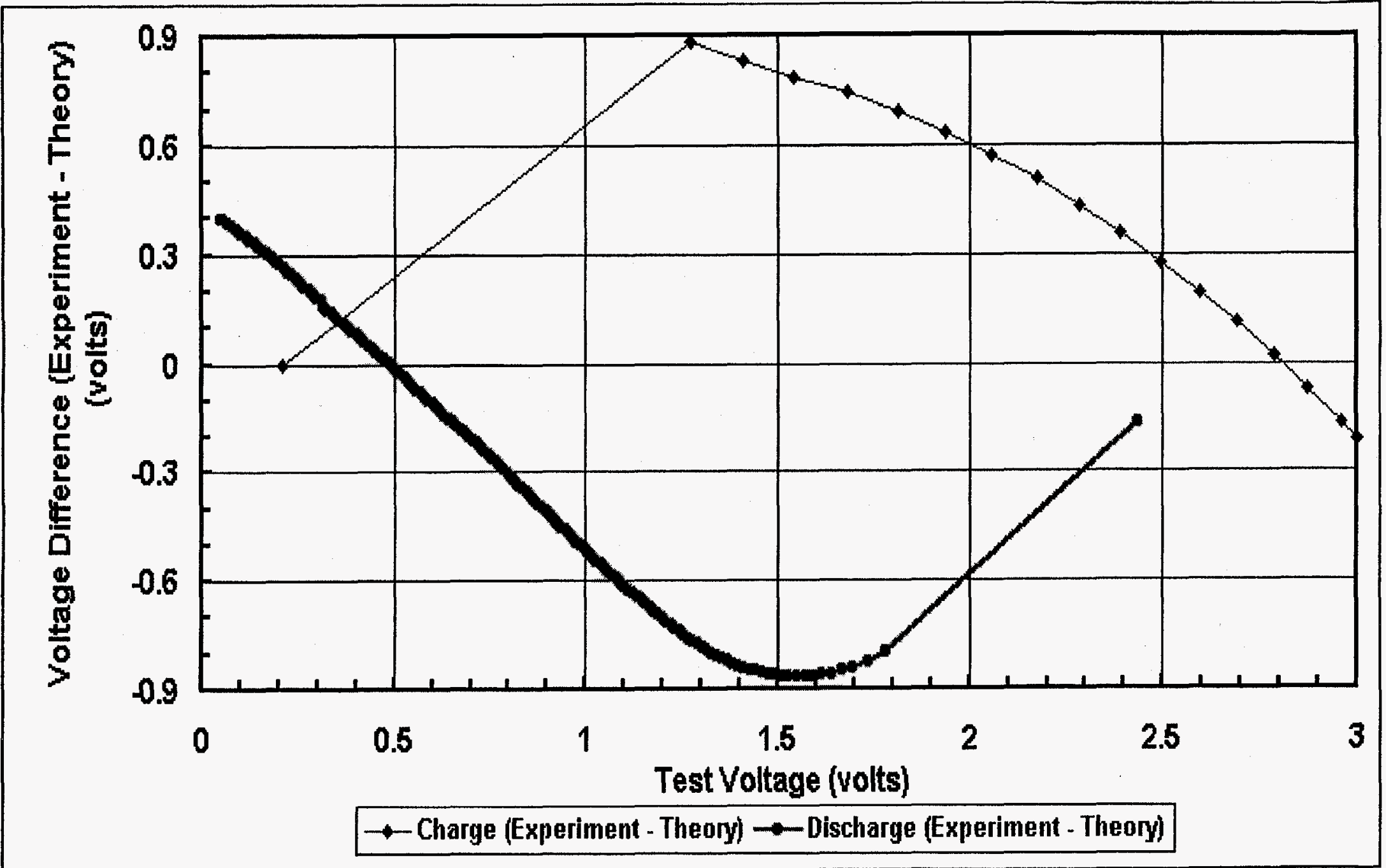

Figure A-62. Voltage difference data for capacitor \#1022 from 250 A constant-current charge/discharge test at $-20^{\circ} \mathrm{C}$. 


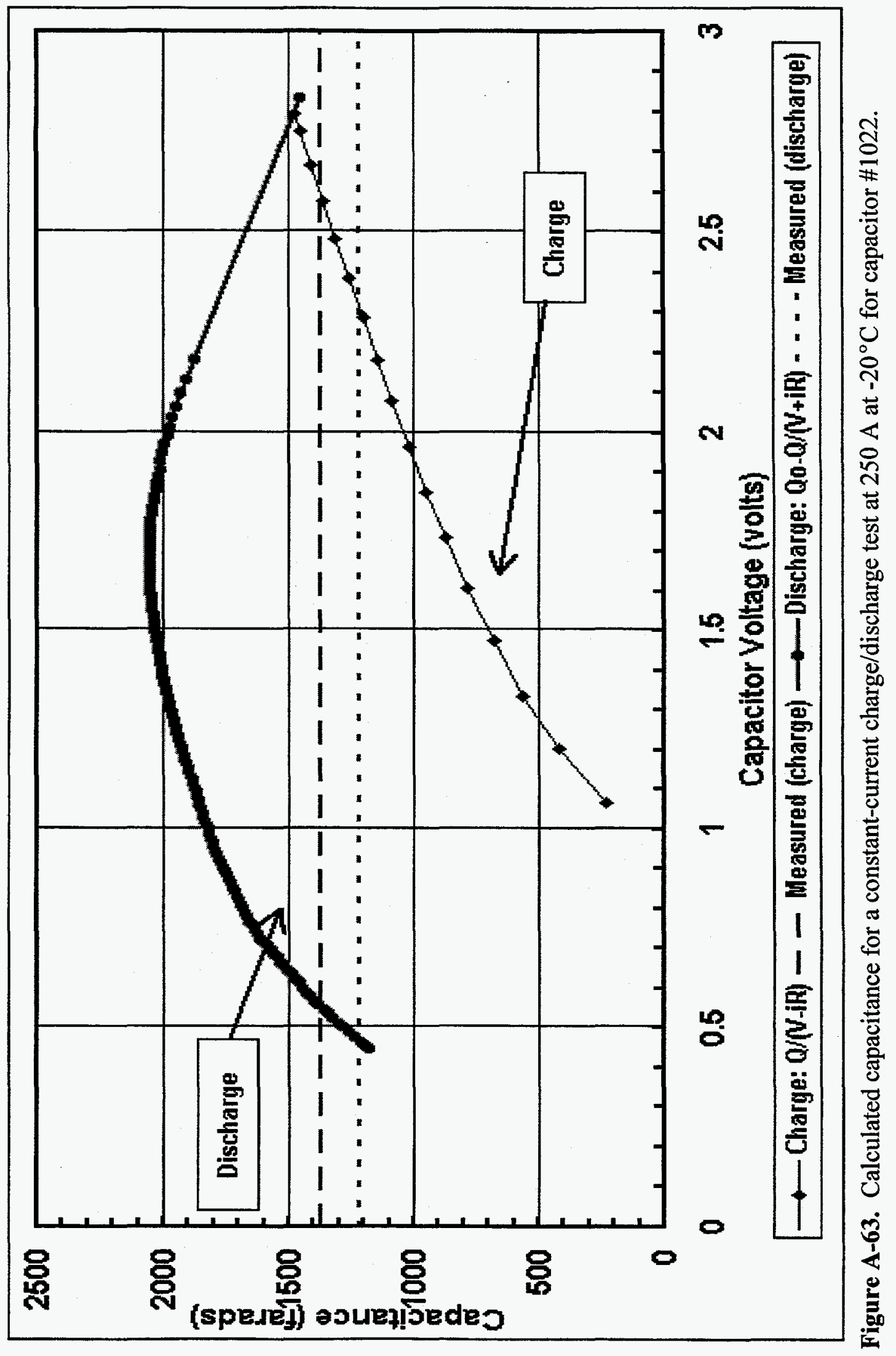




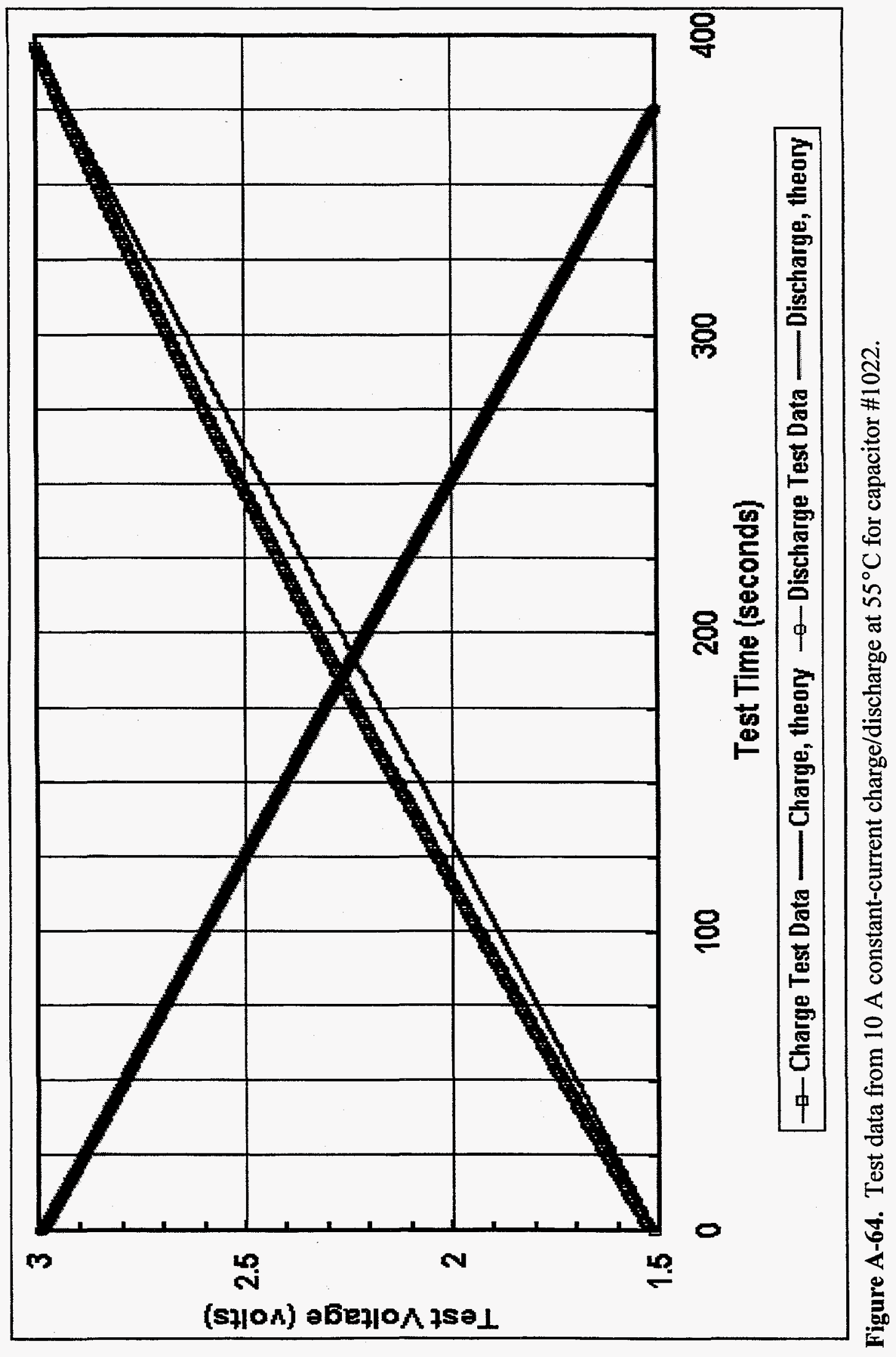




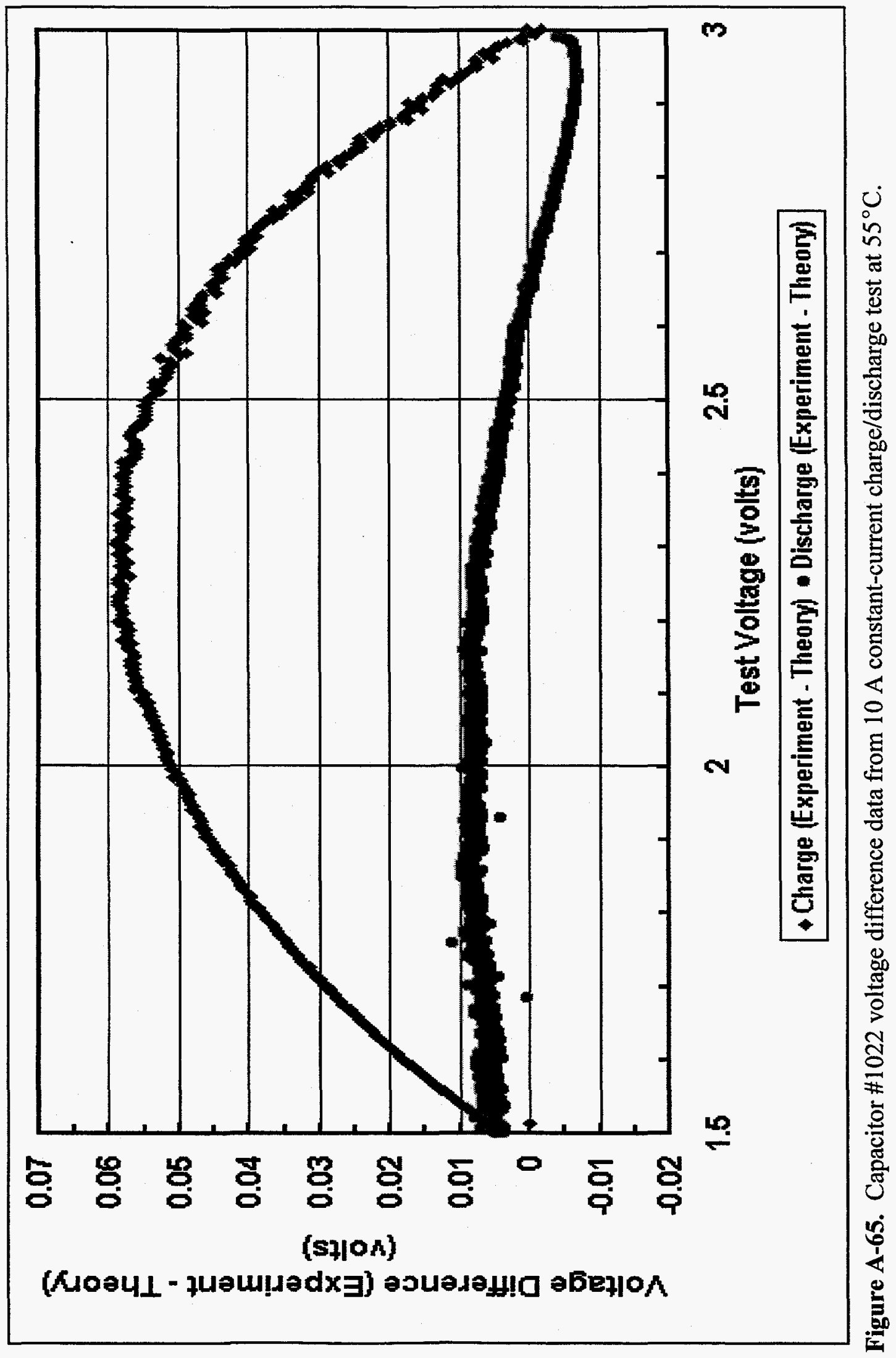




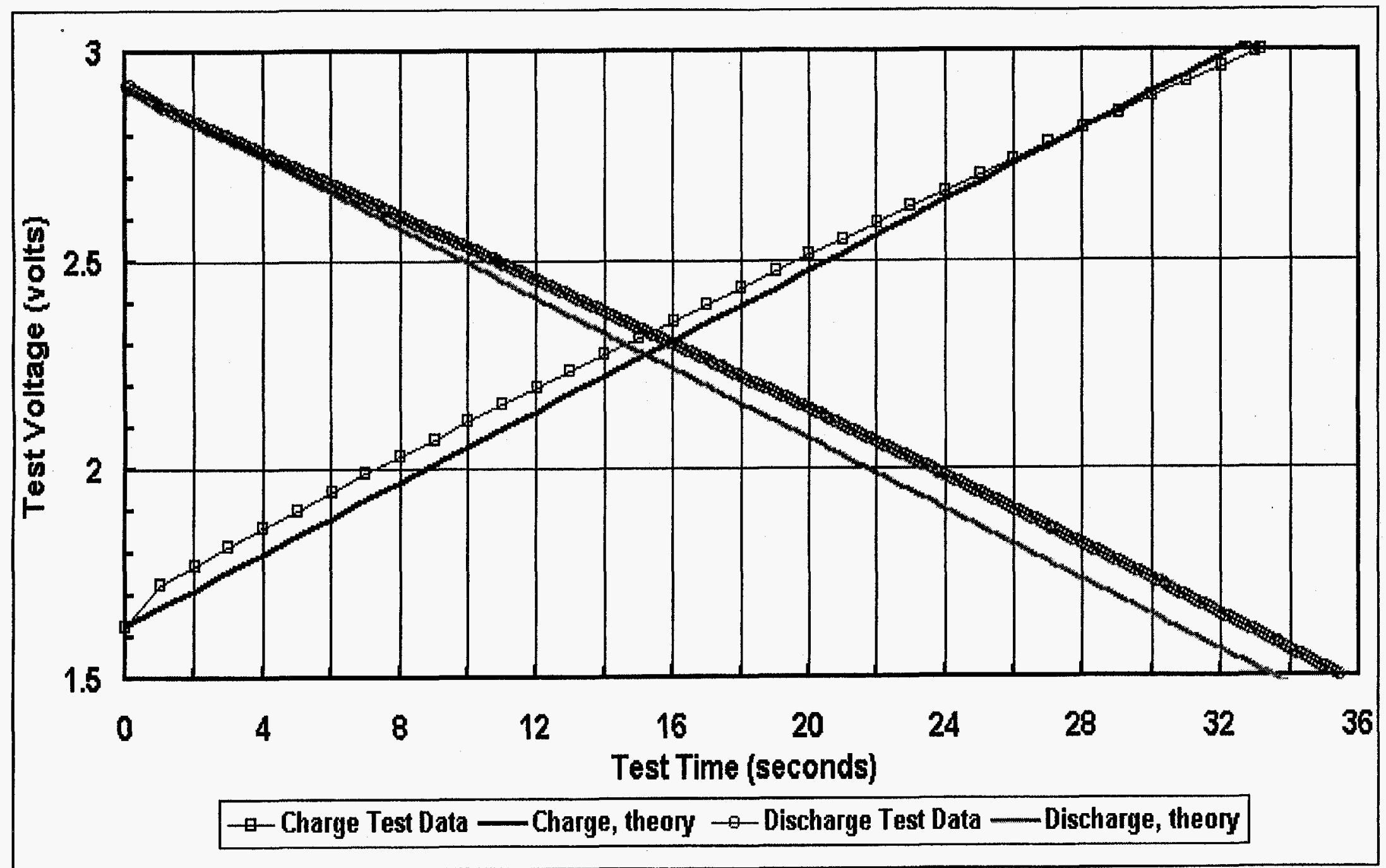

Figure A-66. Data from 100 A constant-current charge/discharge test at $55^{\circ} \mathrm{C}$ for capacitor $\# 1022$. 


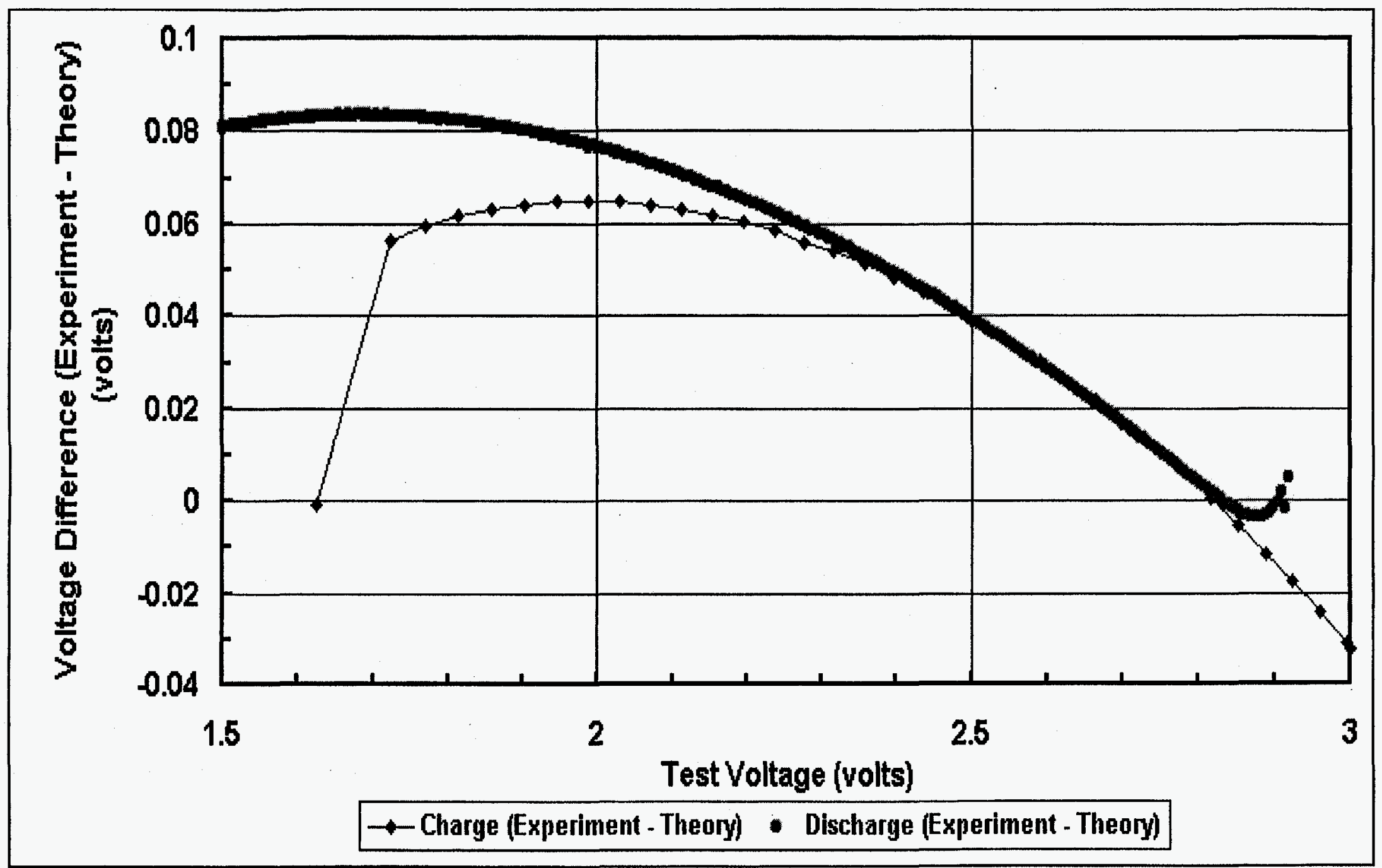

Figure A-67. Voltage difference data from $100 \mathrm{~A}$ constant-current charge/discharge for capacitor \#1022 at $55^{\circ} \mathrm{C}$. 


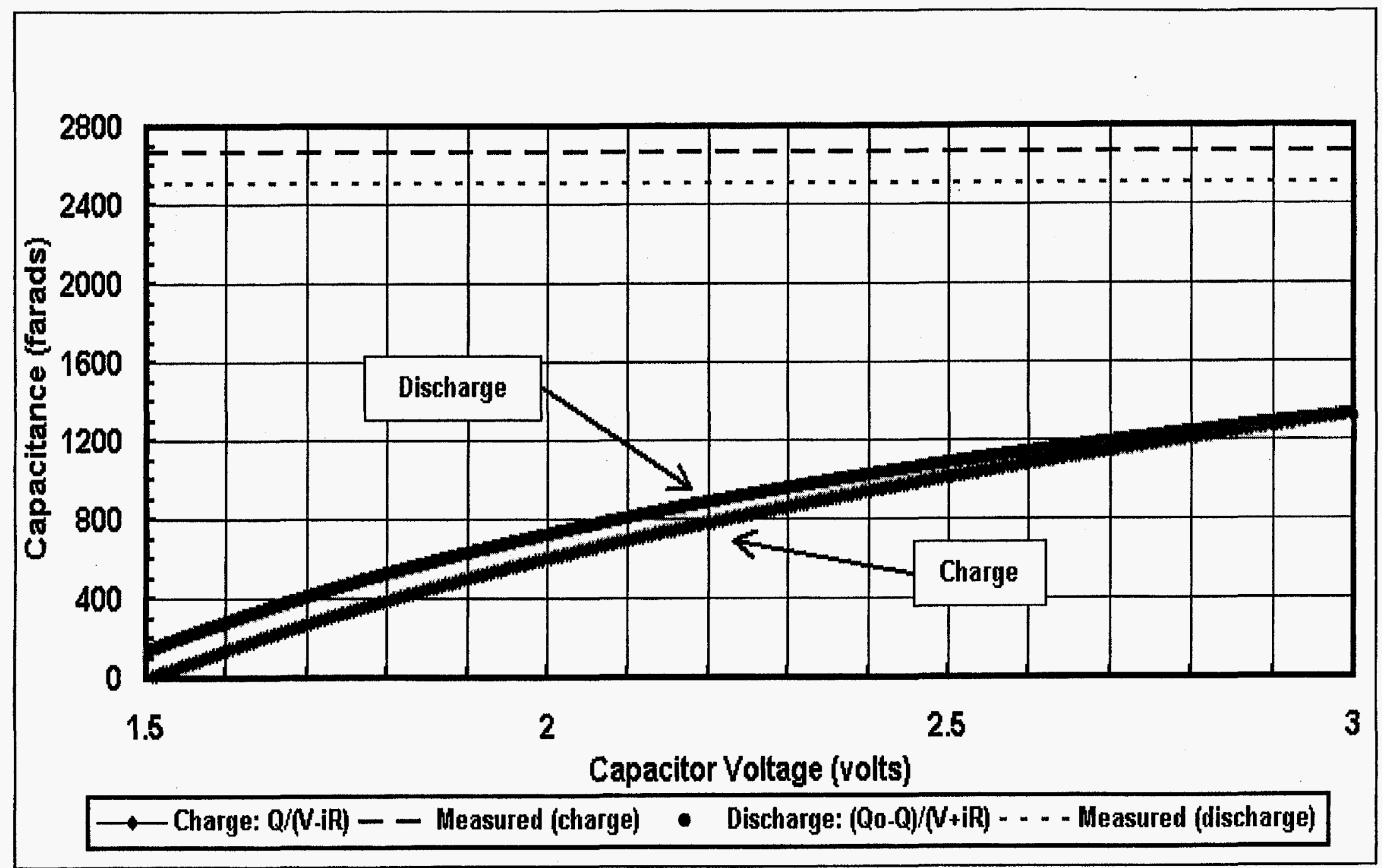

Figure A-68. Calculated capacitance for capacitor $\# 1022$ from $10 \mathrm{~A}$ constant-current test at $55^{\circ} \mathrm{C}$. 


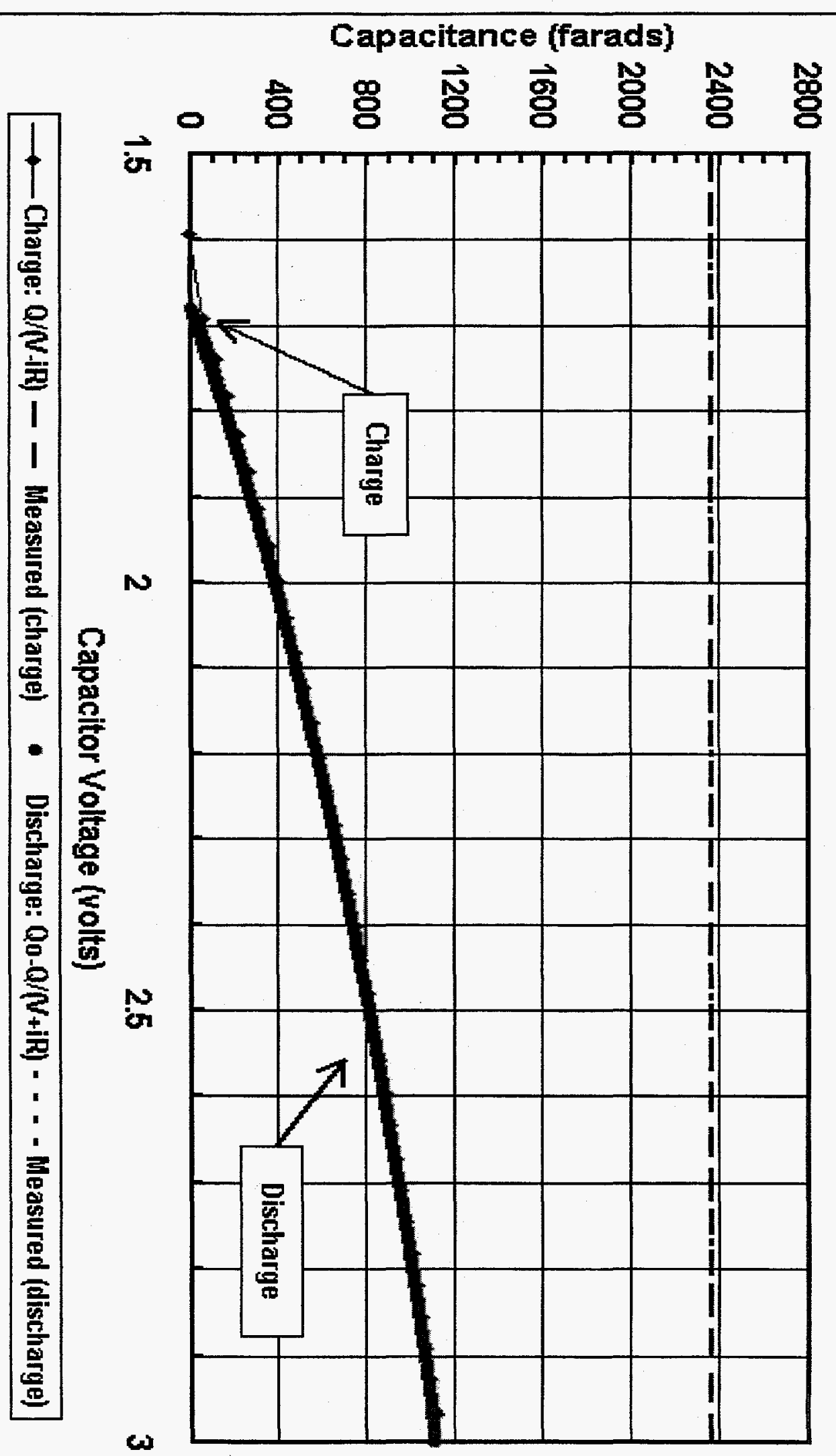




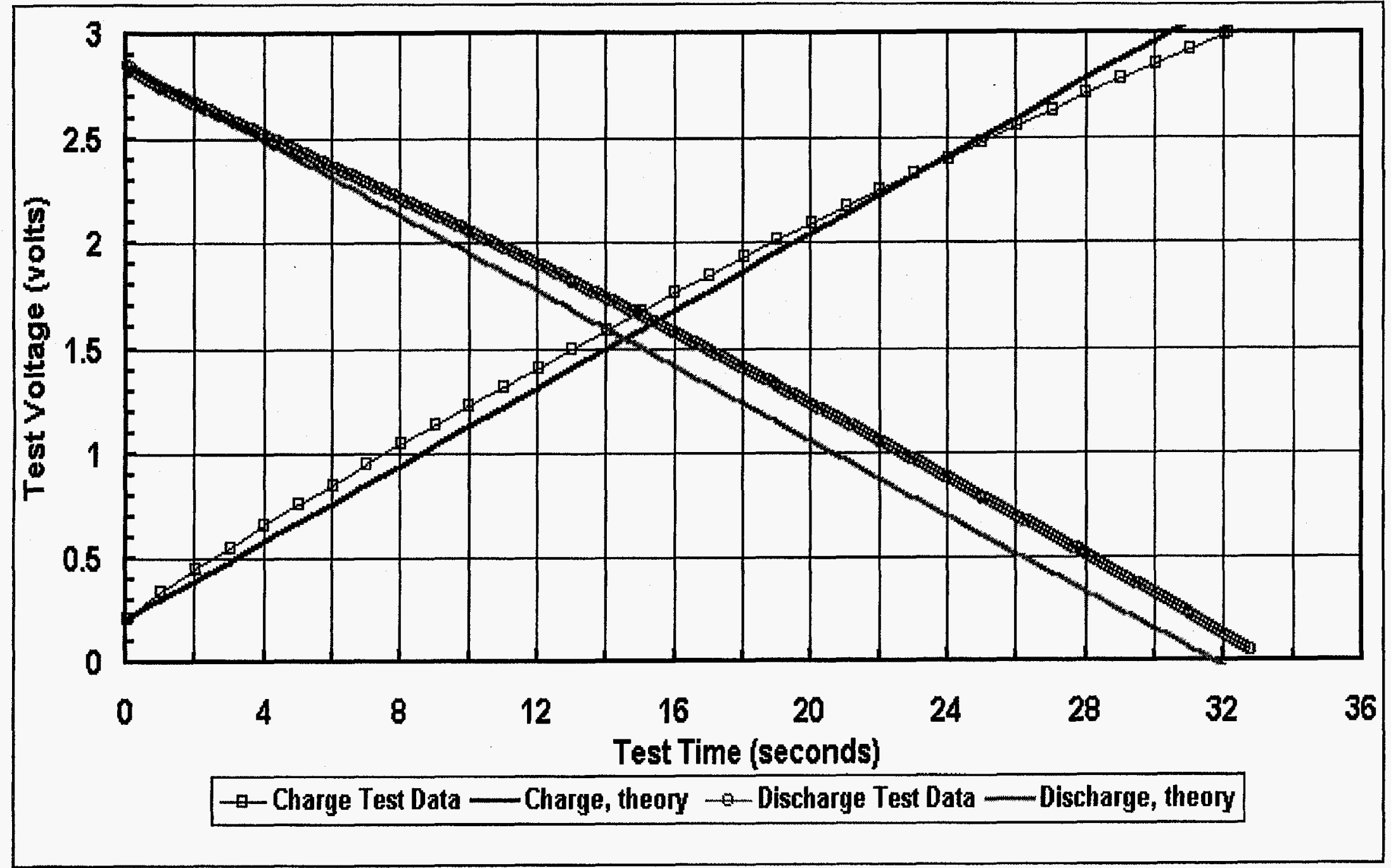

Figure A-70. Constant-current charge/discharge test data for $200 \mathrm{~A}$ at $55^{\circ} \mathrm{C}$ for capacitor $\# 1022$. 


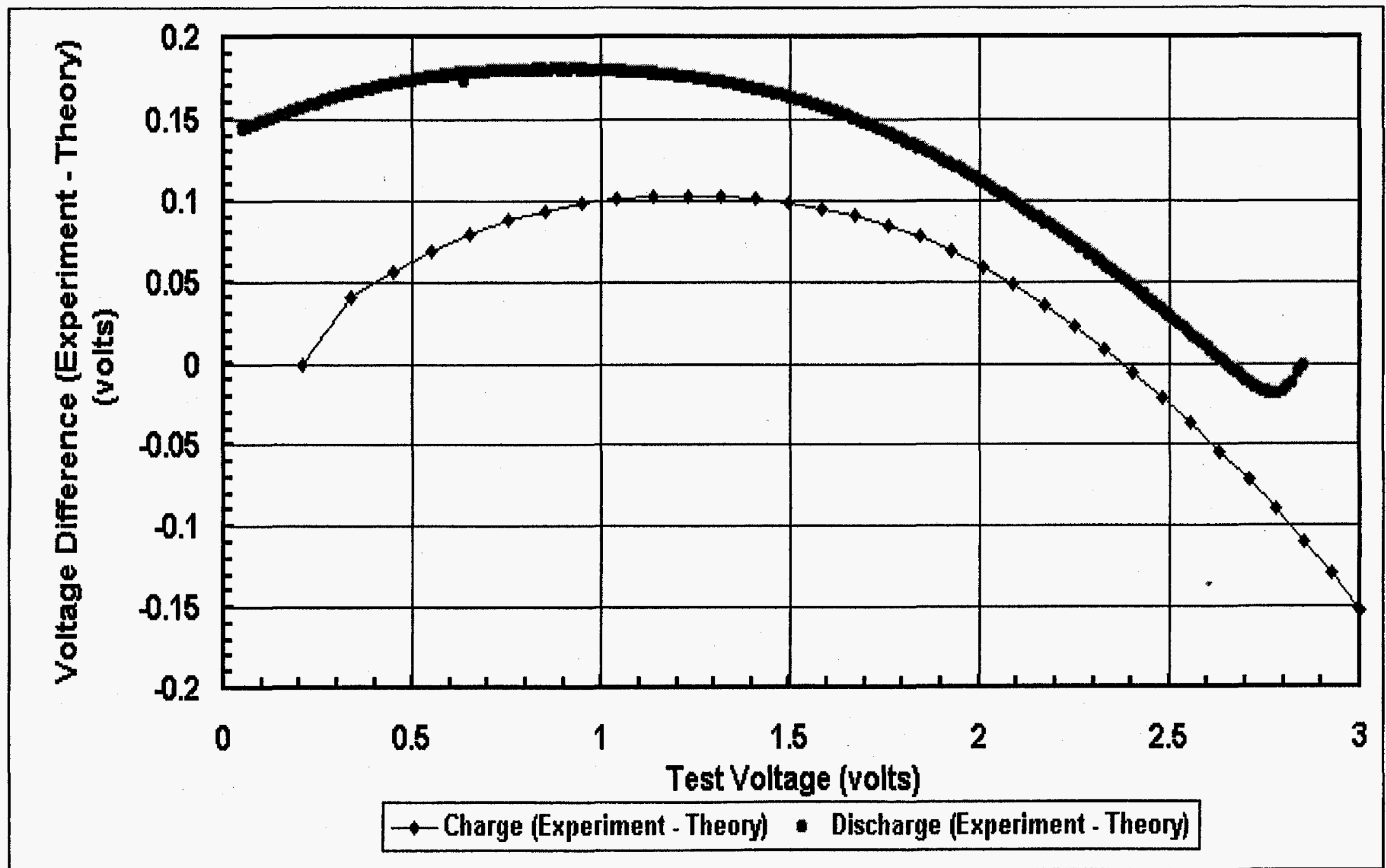

Figure A-71. Voltage difference data for capacitor \#1022 test data from a 200 A constant-current charge/discharge test at $55^{\circ} \mathrm{C}$. 


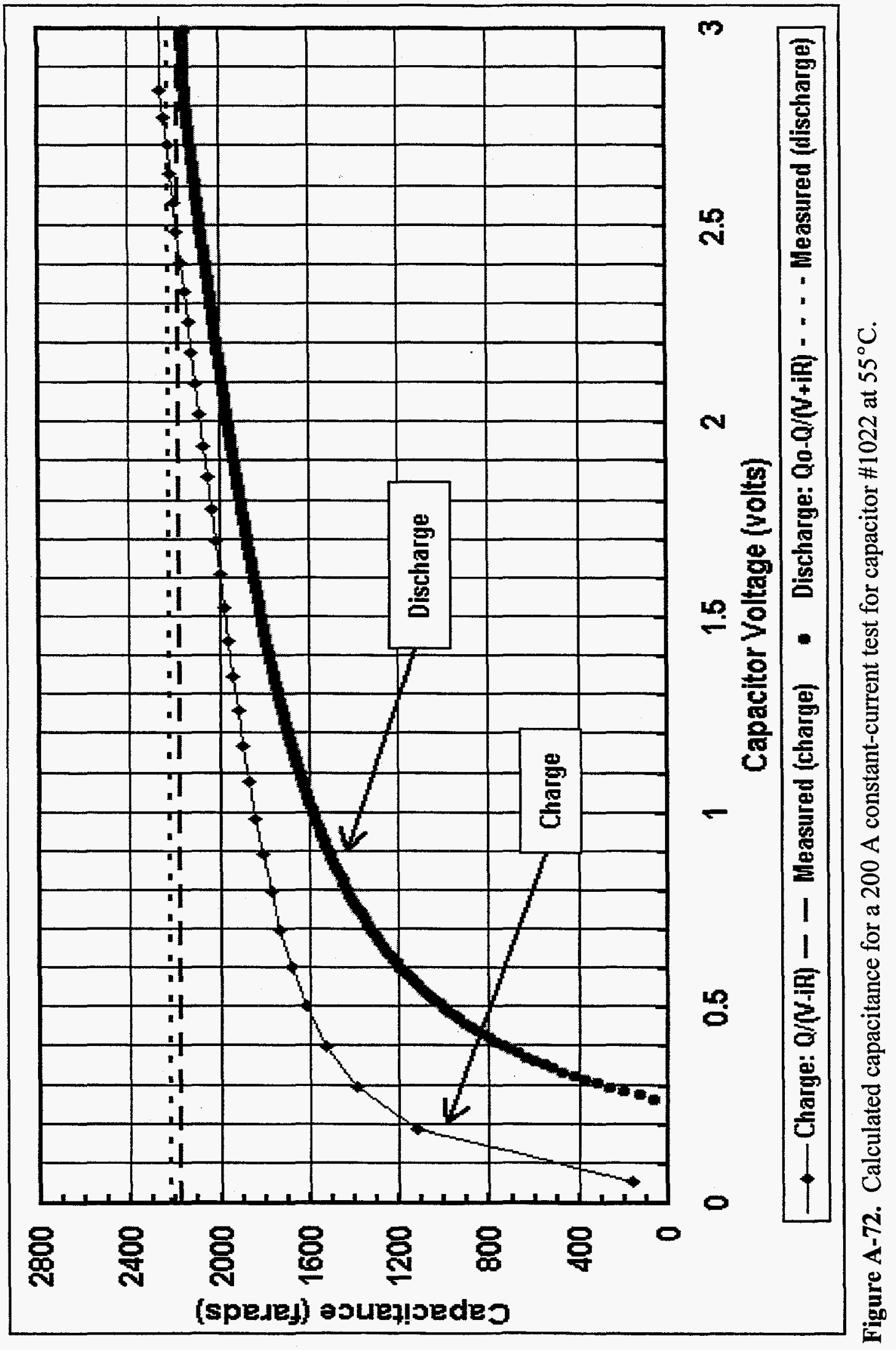




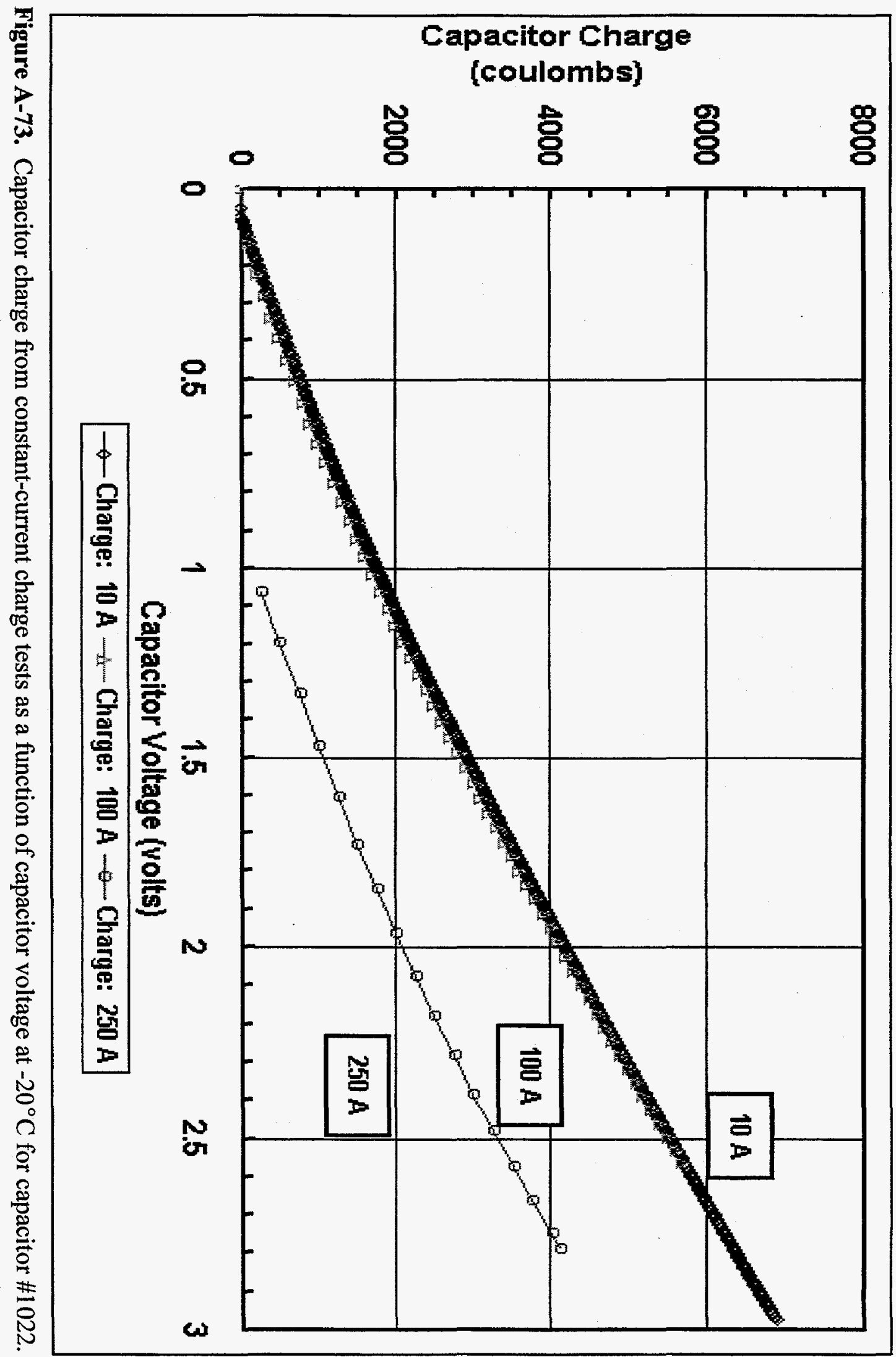




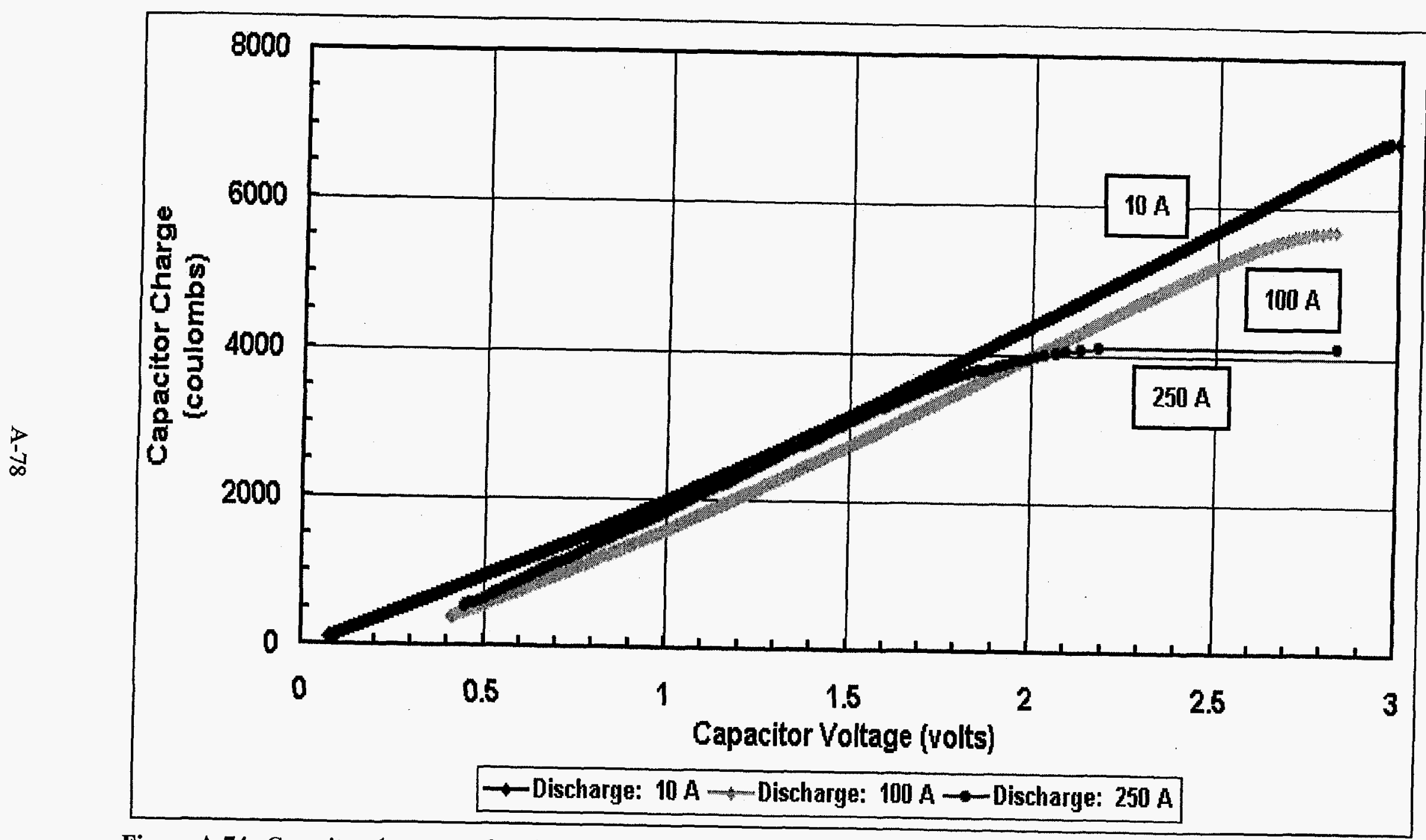
Figure A-74. Capacitor charge as a function of capacitor voltage for capacitor $\# 1022$ from constant-current discharge test
at $-20^{\circ} \mathrm{C}$. 


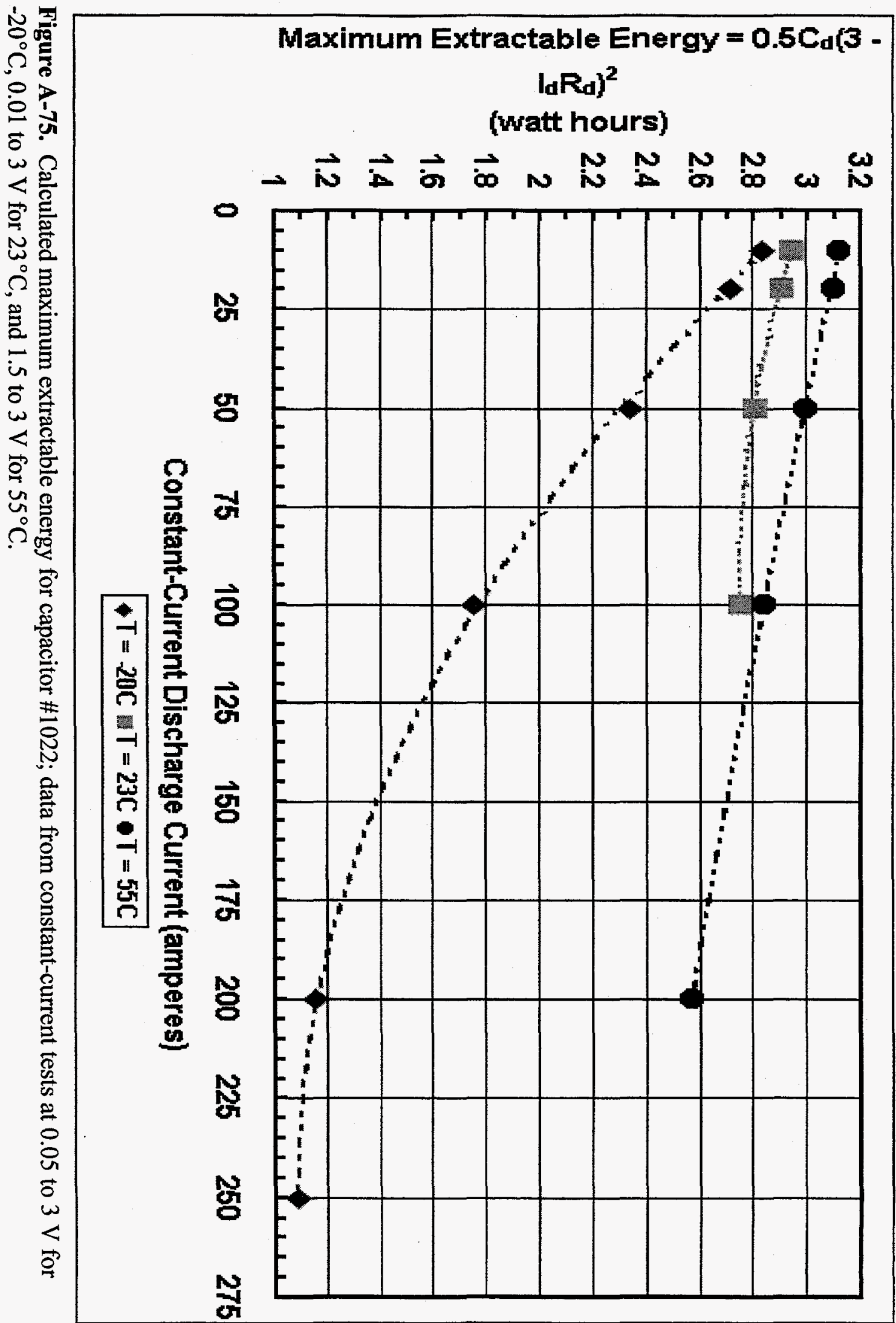




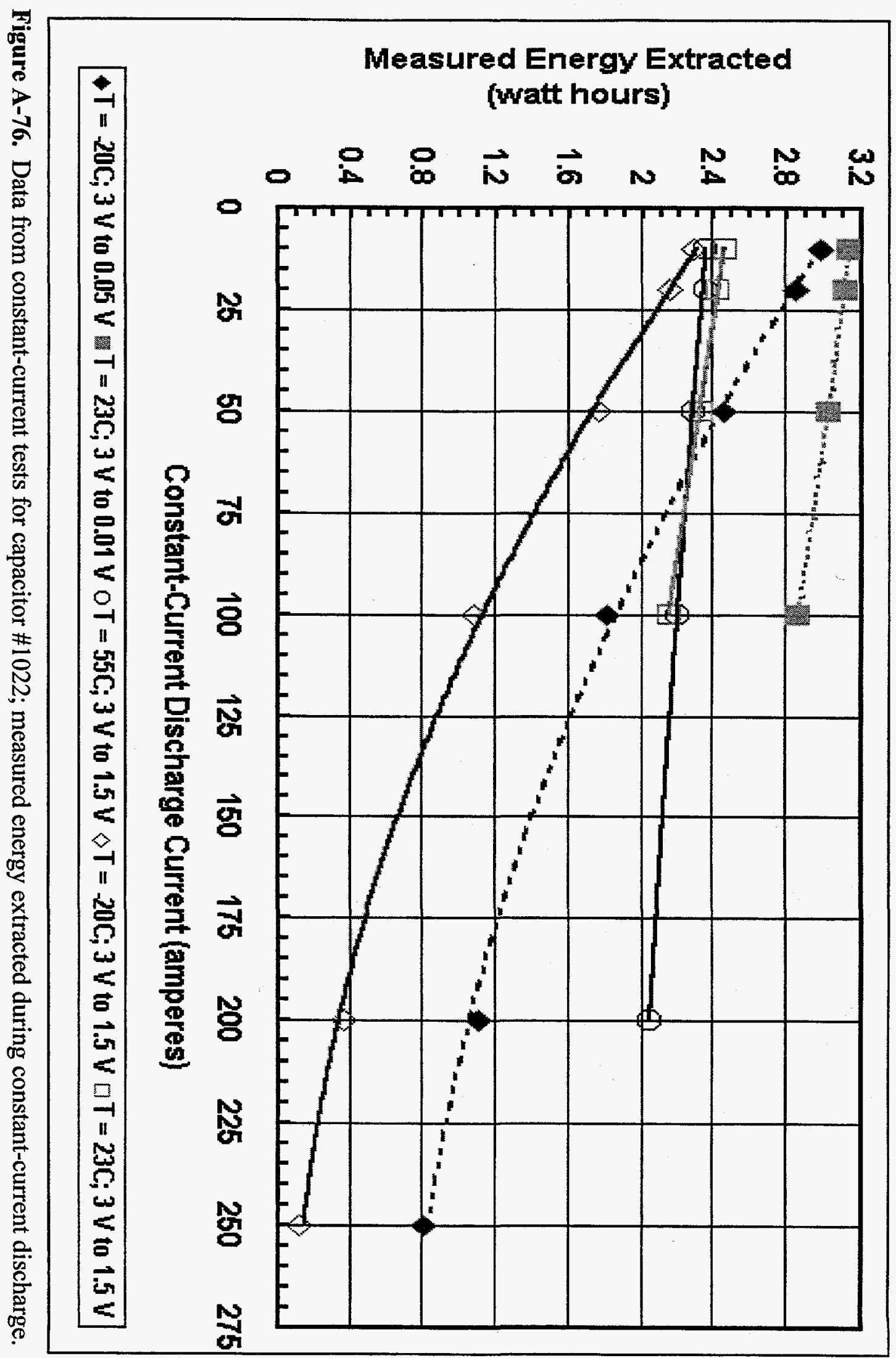




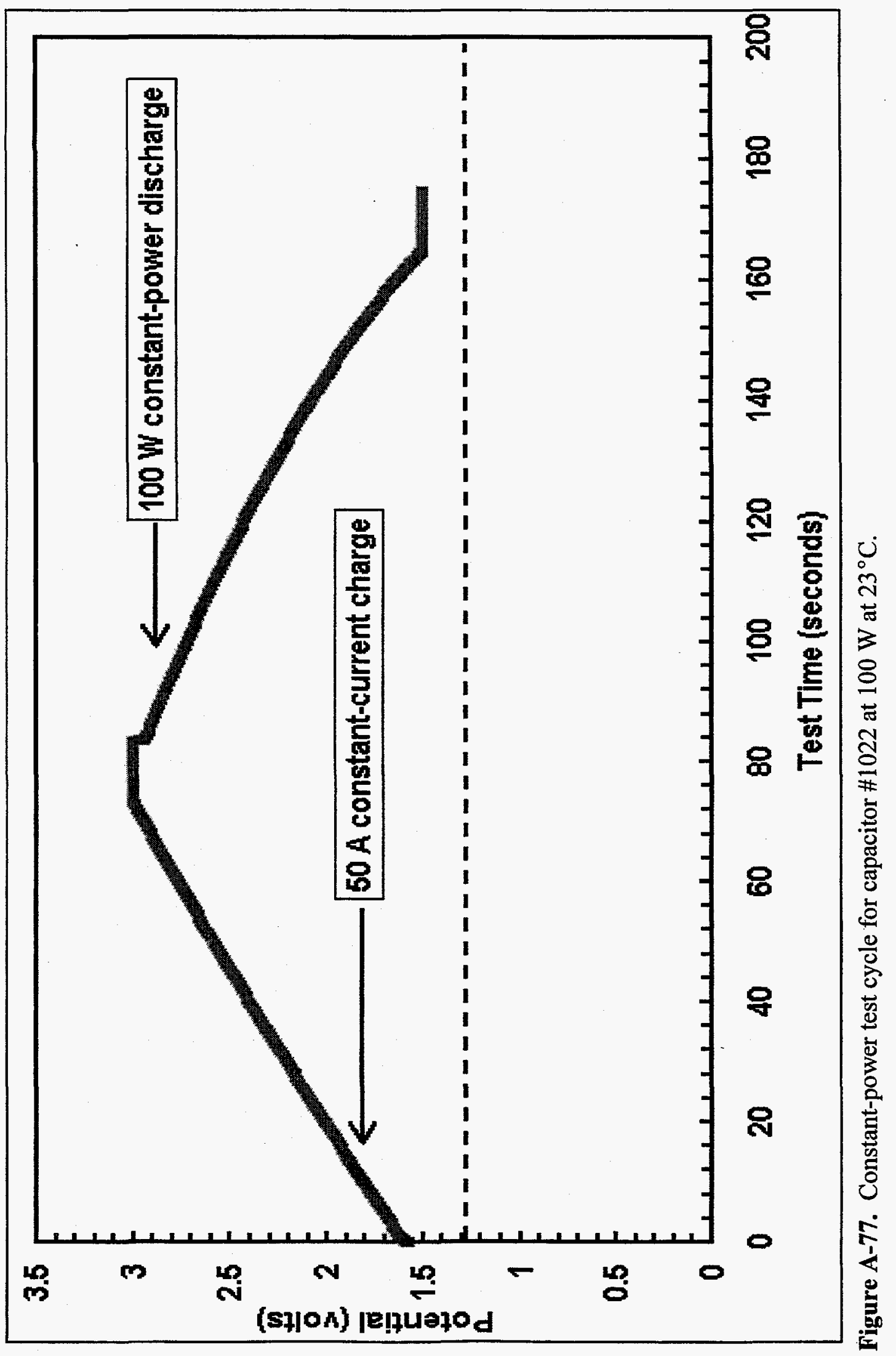




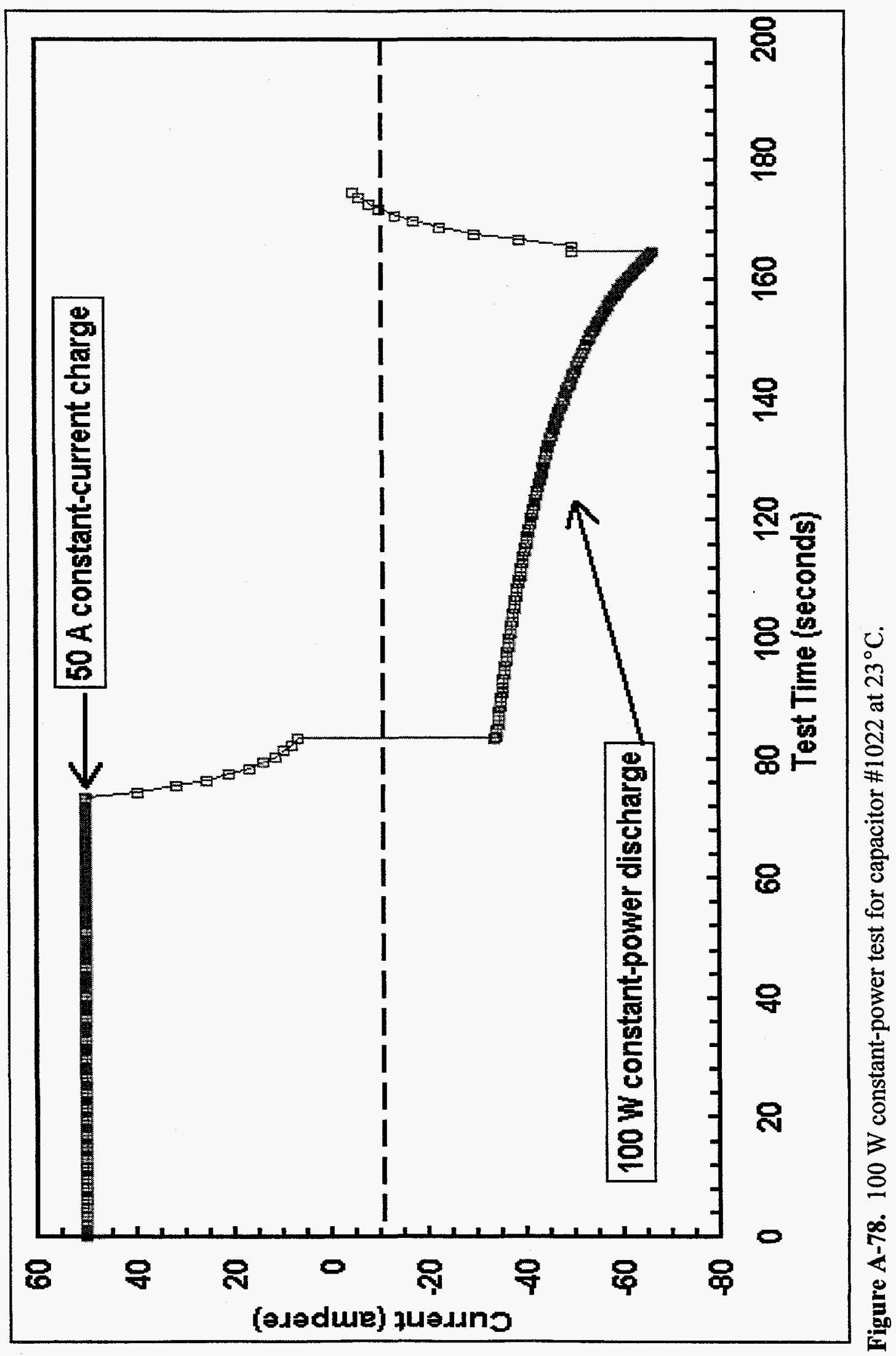




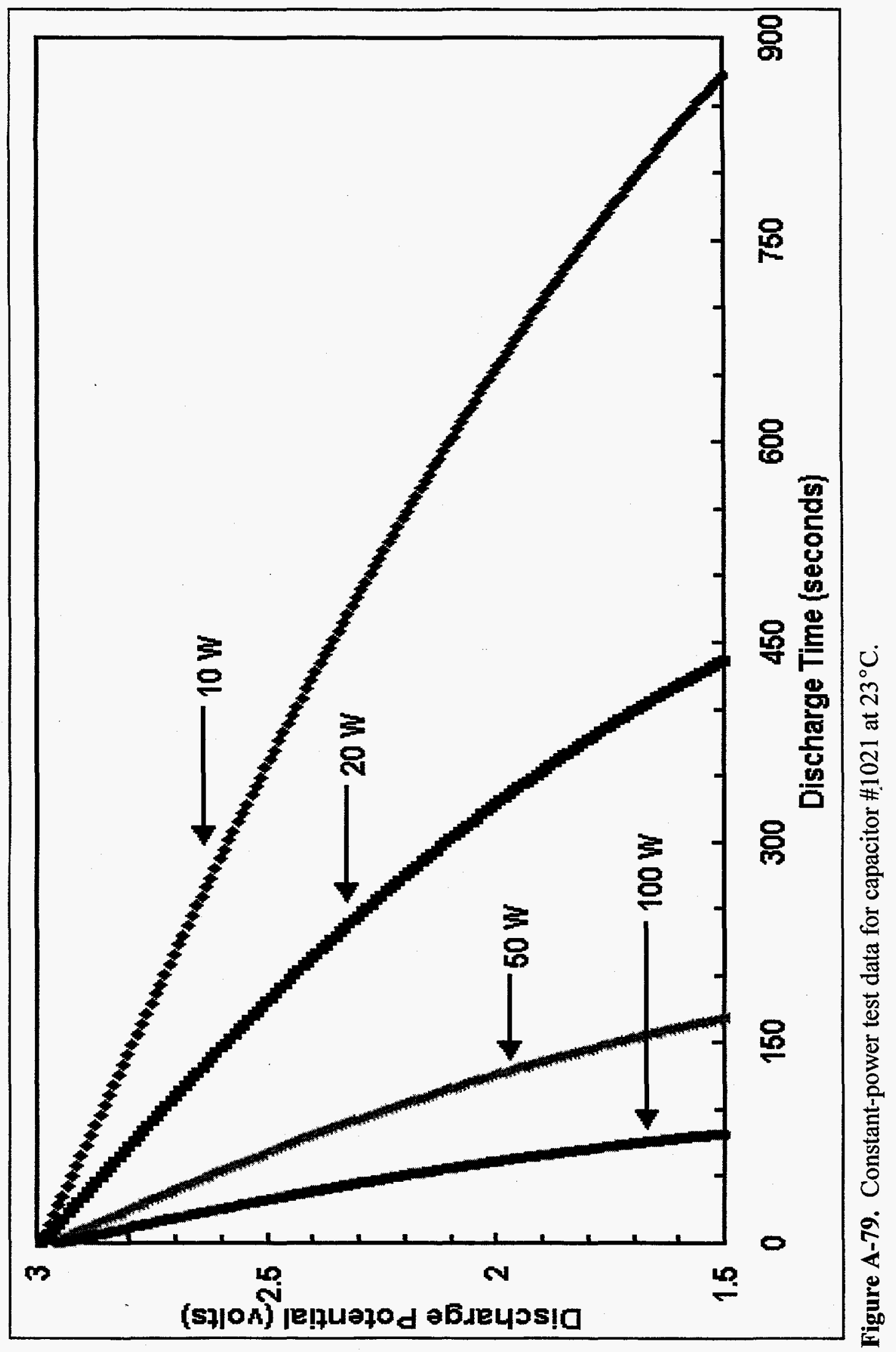




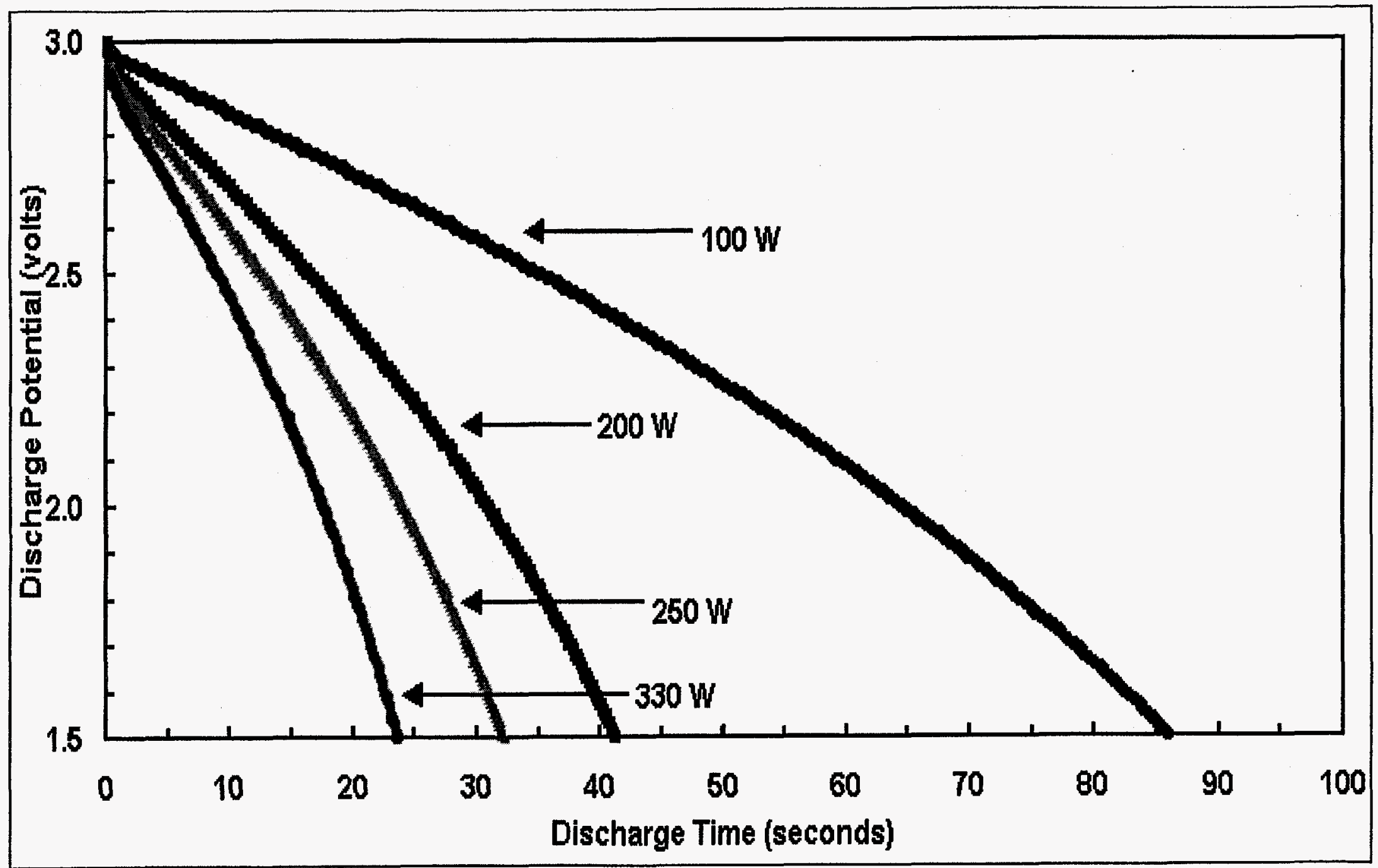

Figure A-80. Data from constant-power test for capacitor $\# 1021$ at $23^{\circ} \mathrm{C}$. 


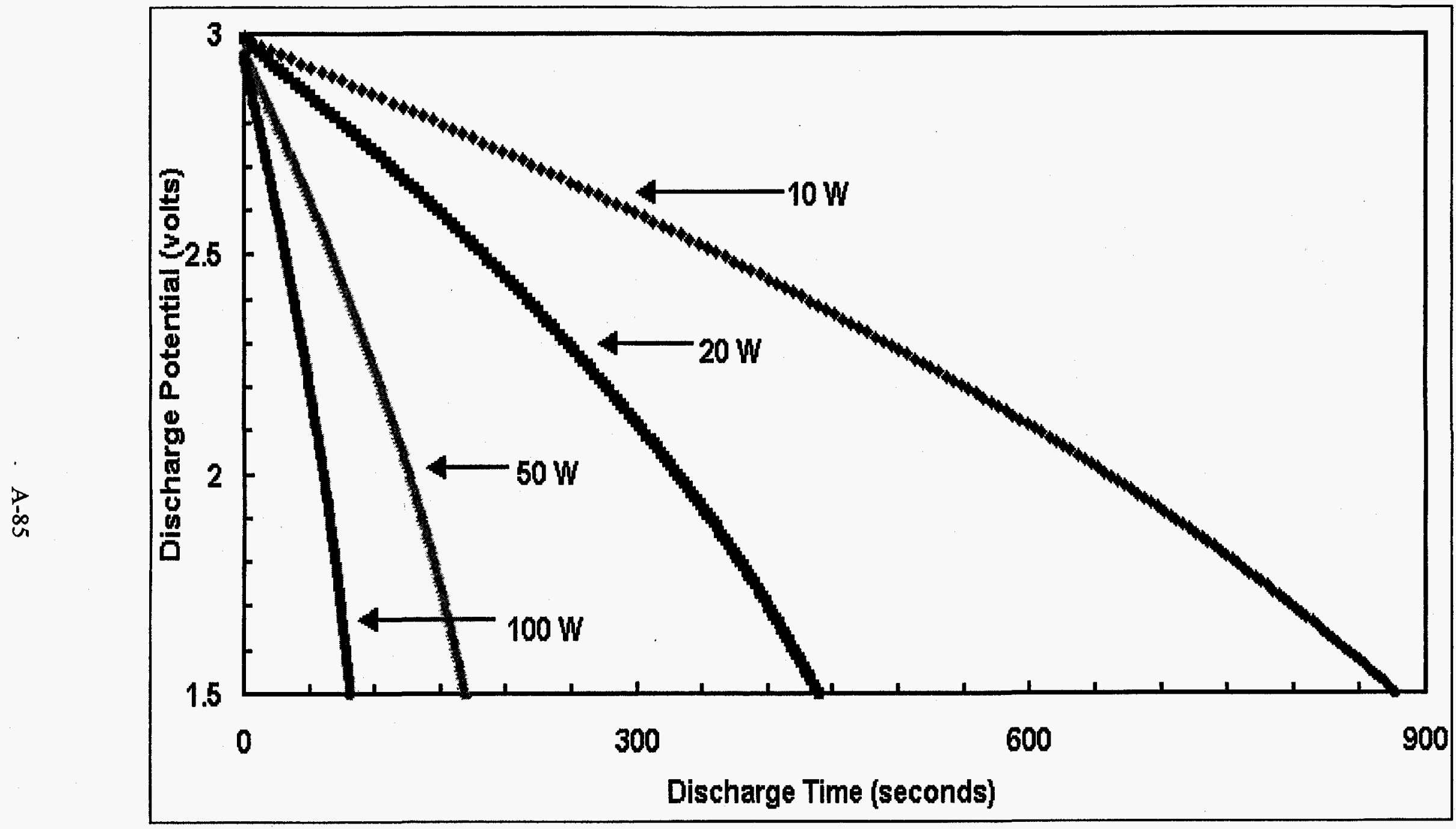

Figure A-81. Data from constant-power test for capacitor $\# 1022$ at $23^{\circ} \mathrm{C}$. 


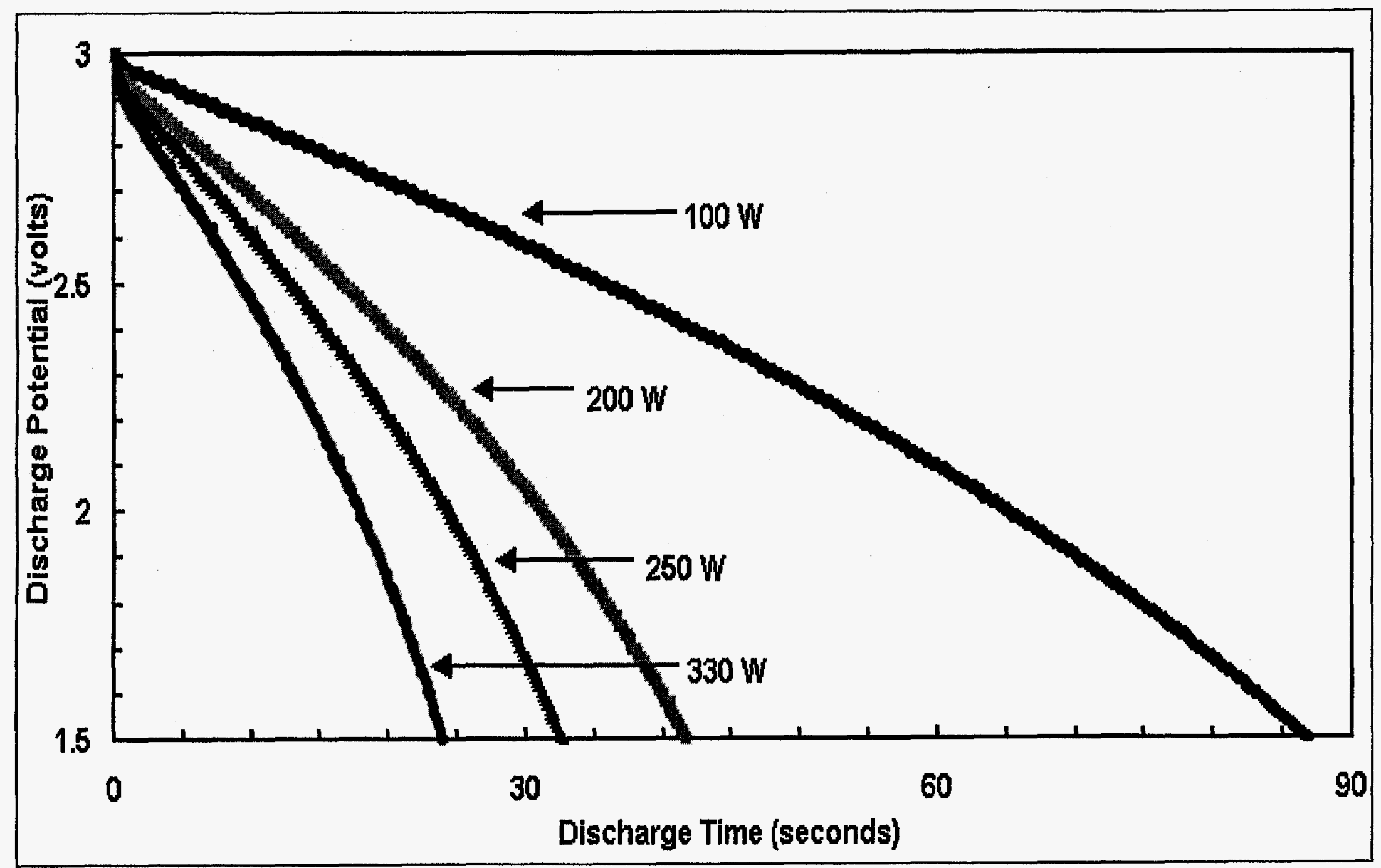

Figure A-82. Constant-power test data for capacitor $\# 1022$ at $23^{\circ} \mathrm{C}$. 


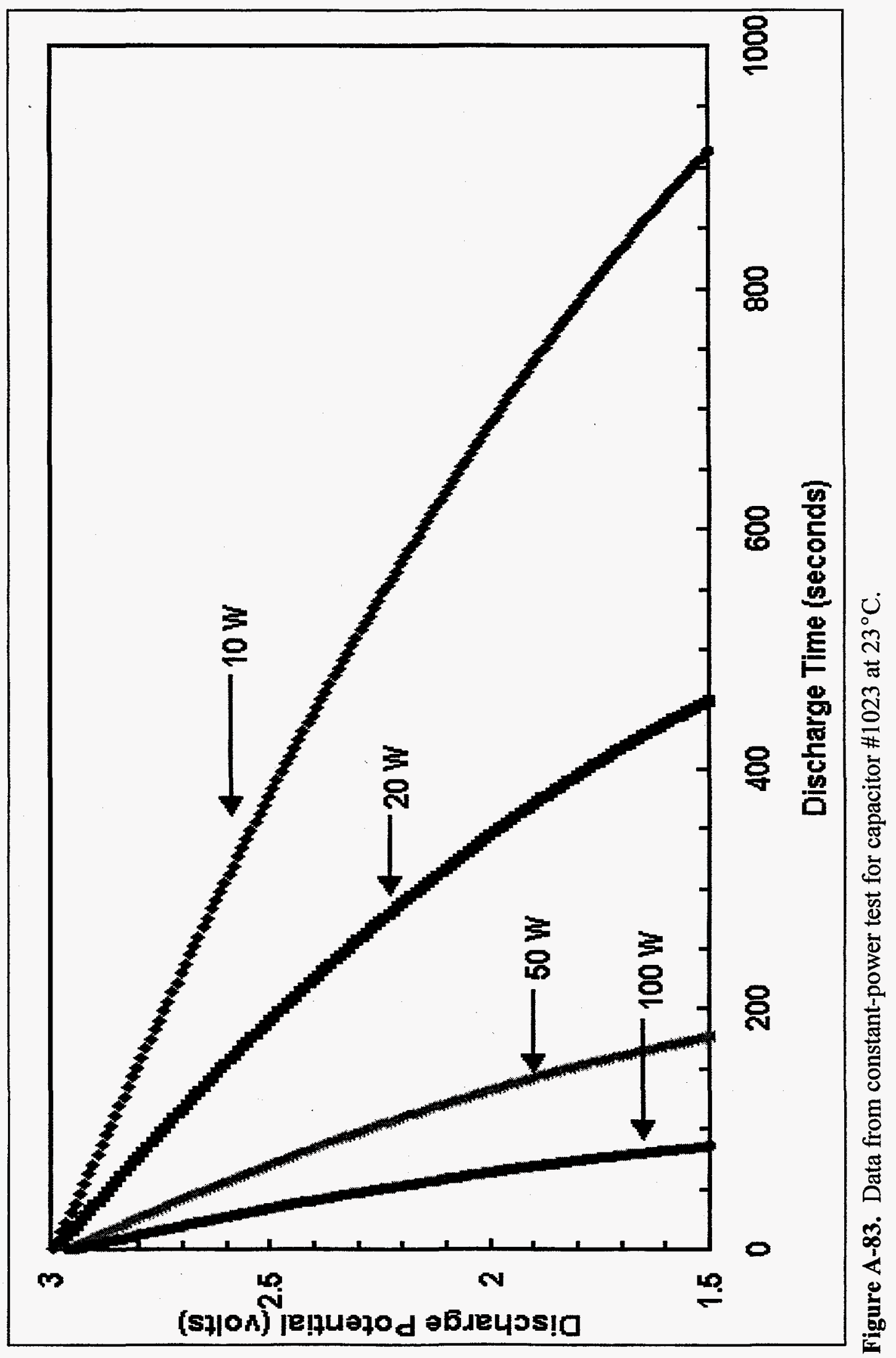




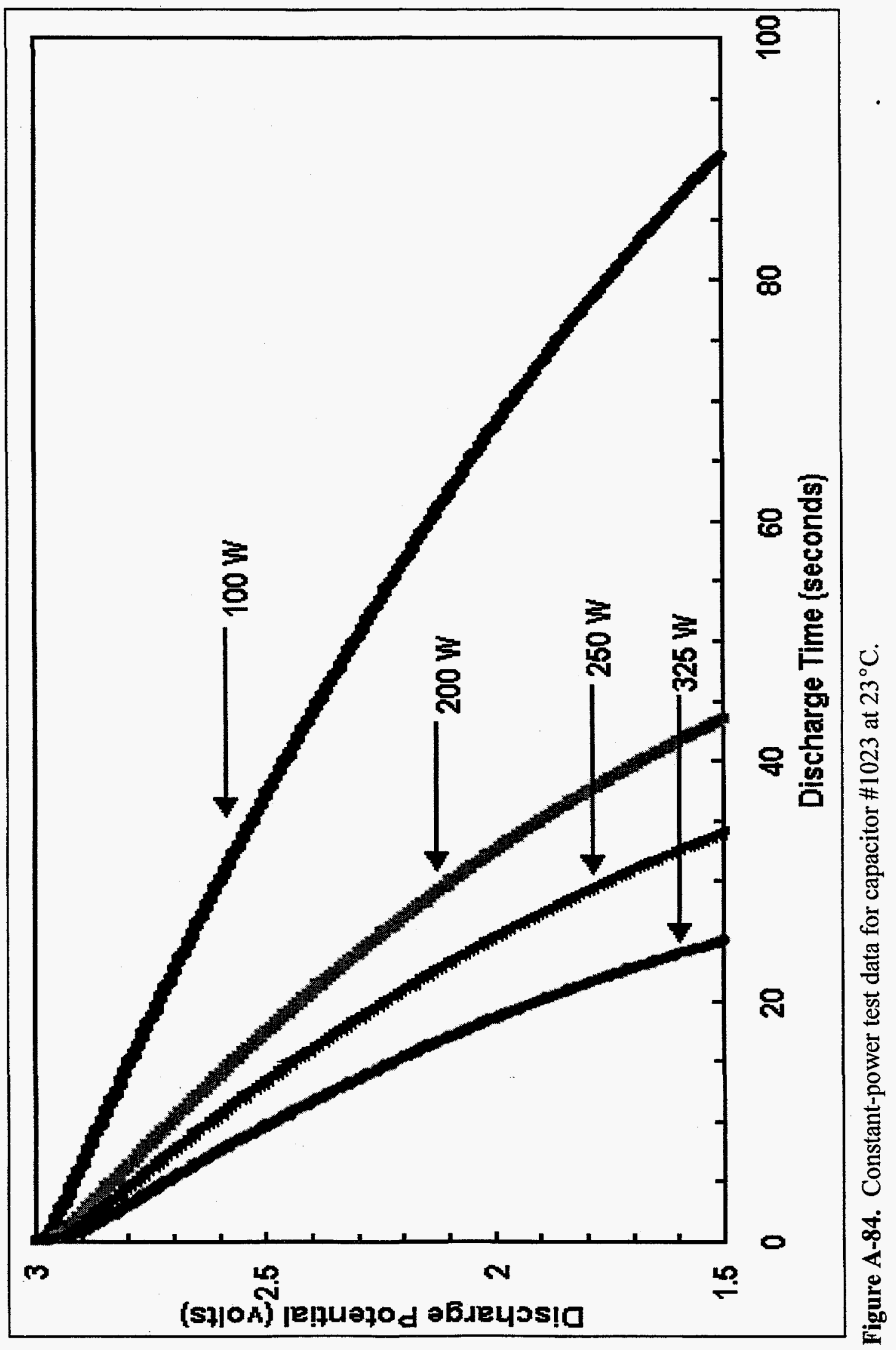




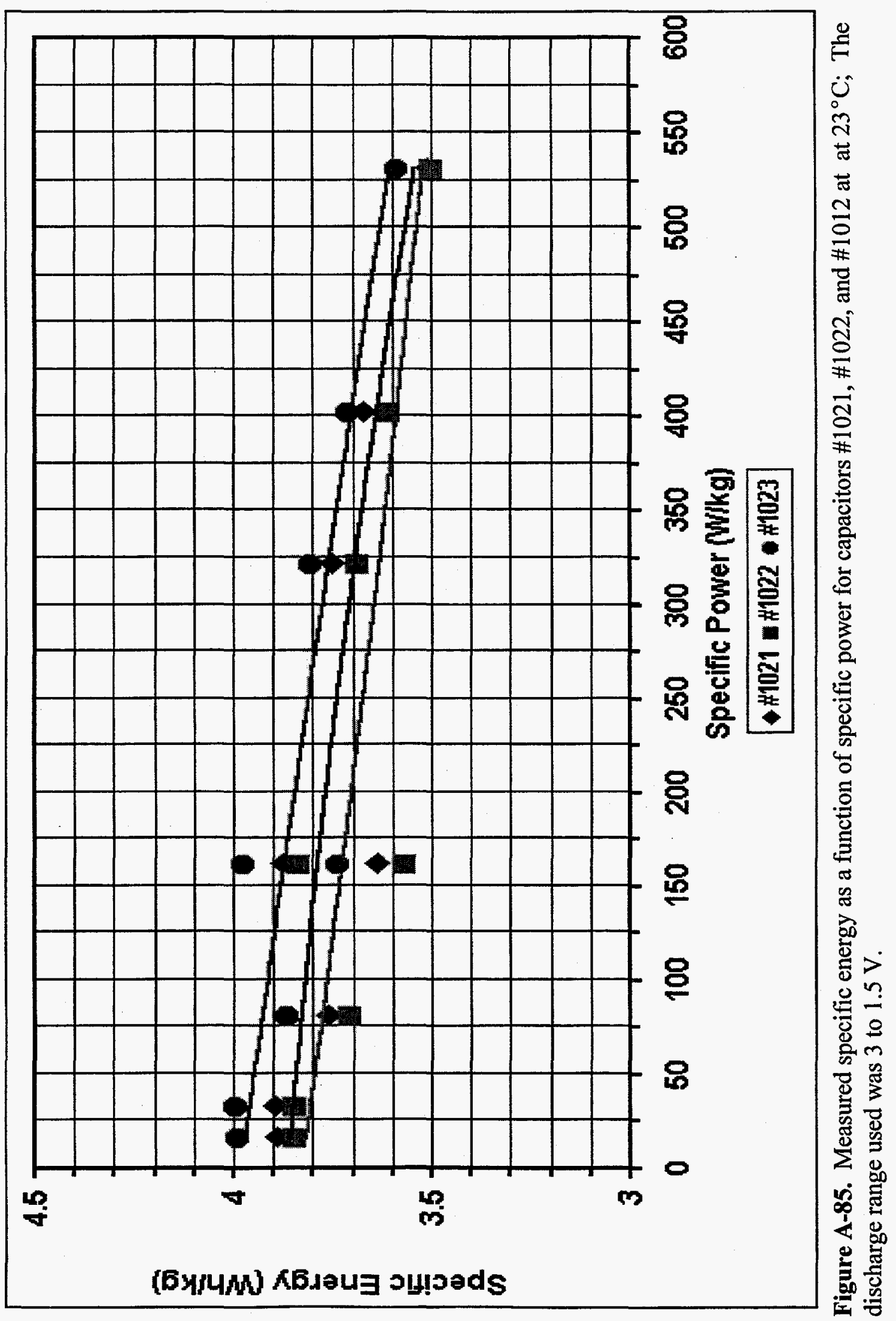




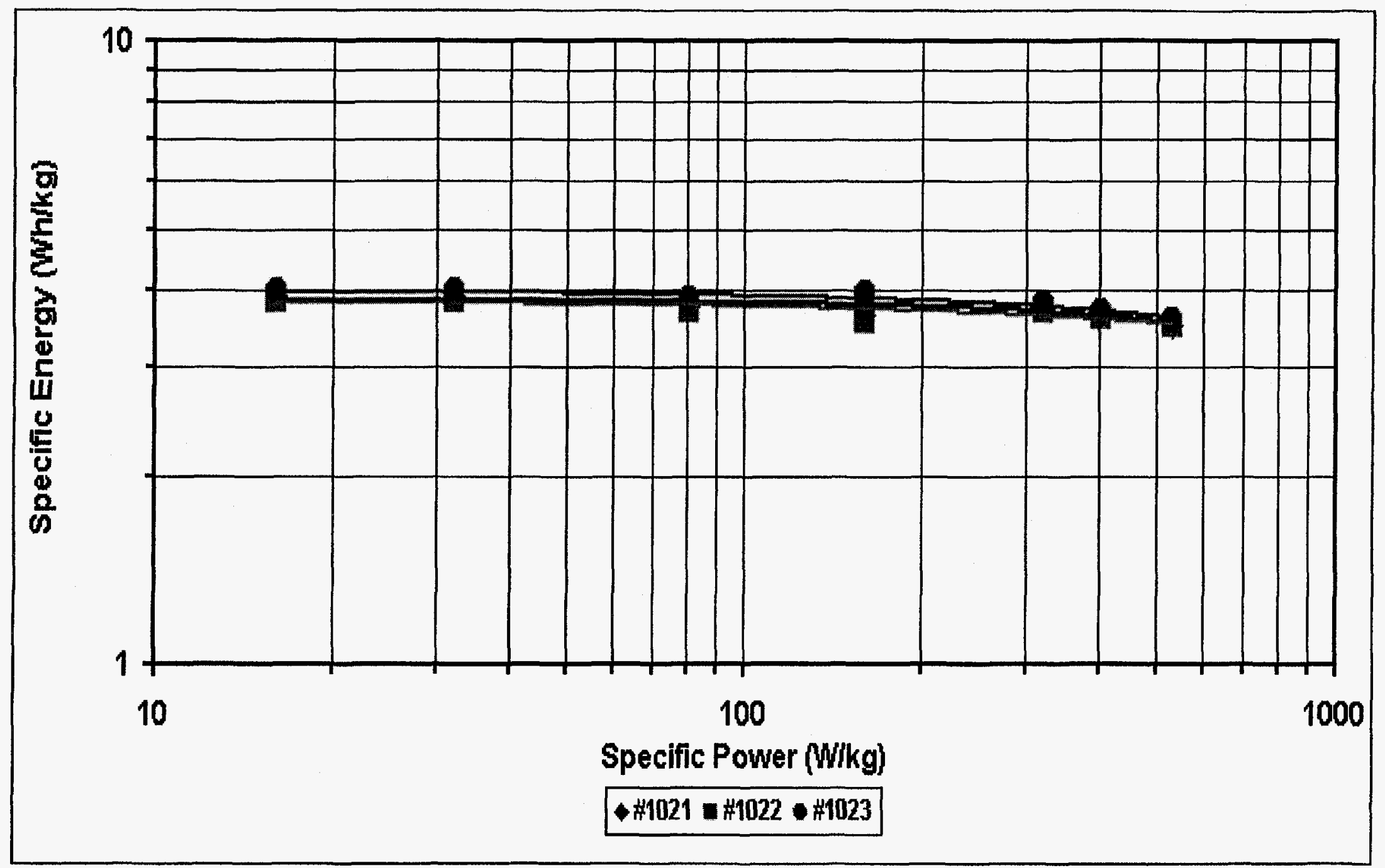

Figure A-86. Measured specific energy as a function of specific power for capacitors \#1021, \#1022, and $\# 1023$ at $23^{\circ} \mathrm{C}$; a 3 to $1.5 \mathrm{~V}$ discharge range was used. 


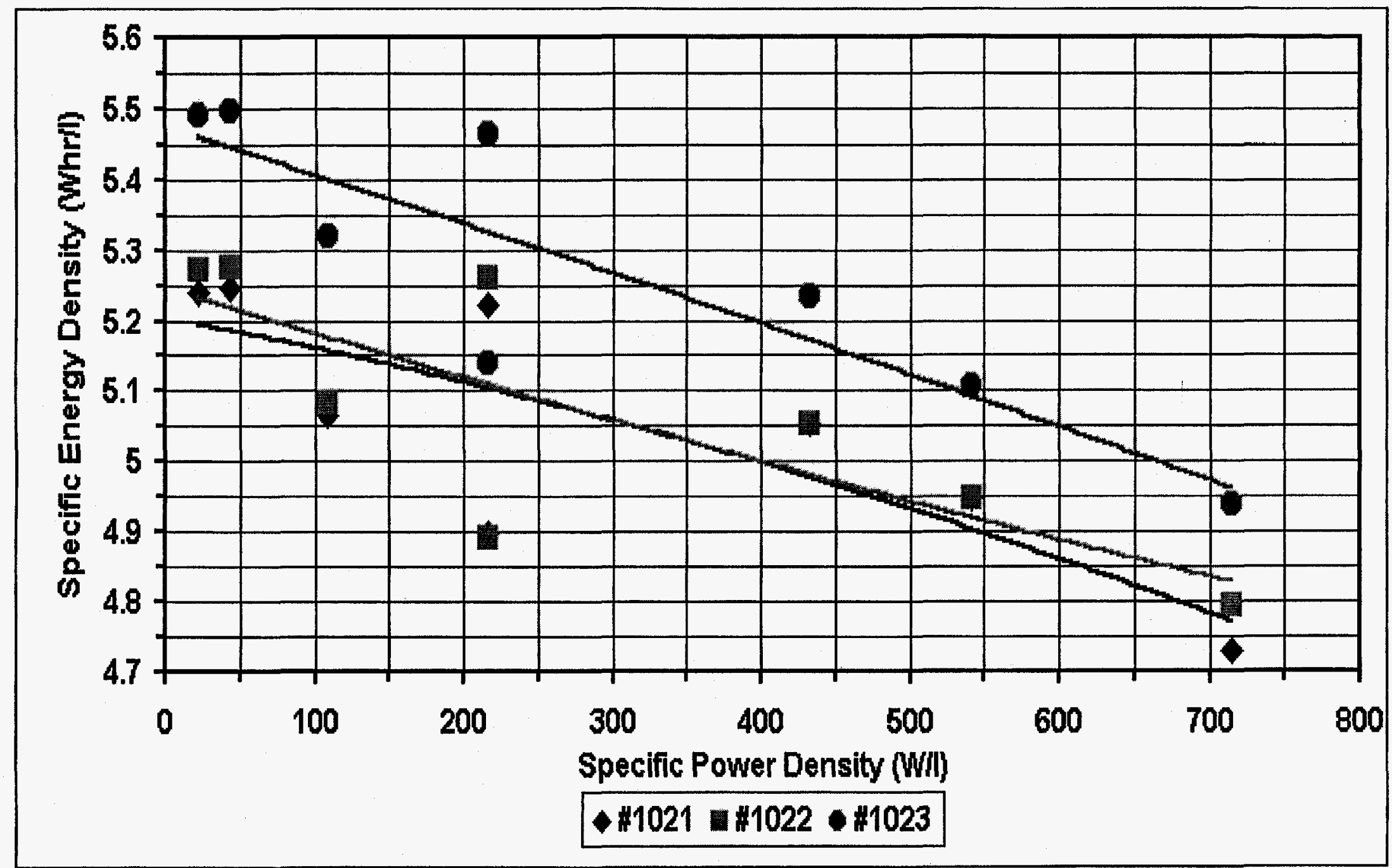

Figure A-87. Specific energy volumetric density as a function of specific power volumetric density for capacitors \#1021, \#1022, and \#1023 at $23^{\circ} \mathrm{C}$. A discharge voltage range of 3 to $1.5 \mathrm{~V}$ was used in the tests. 


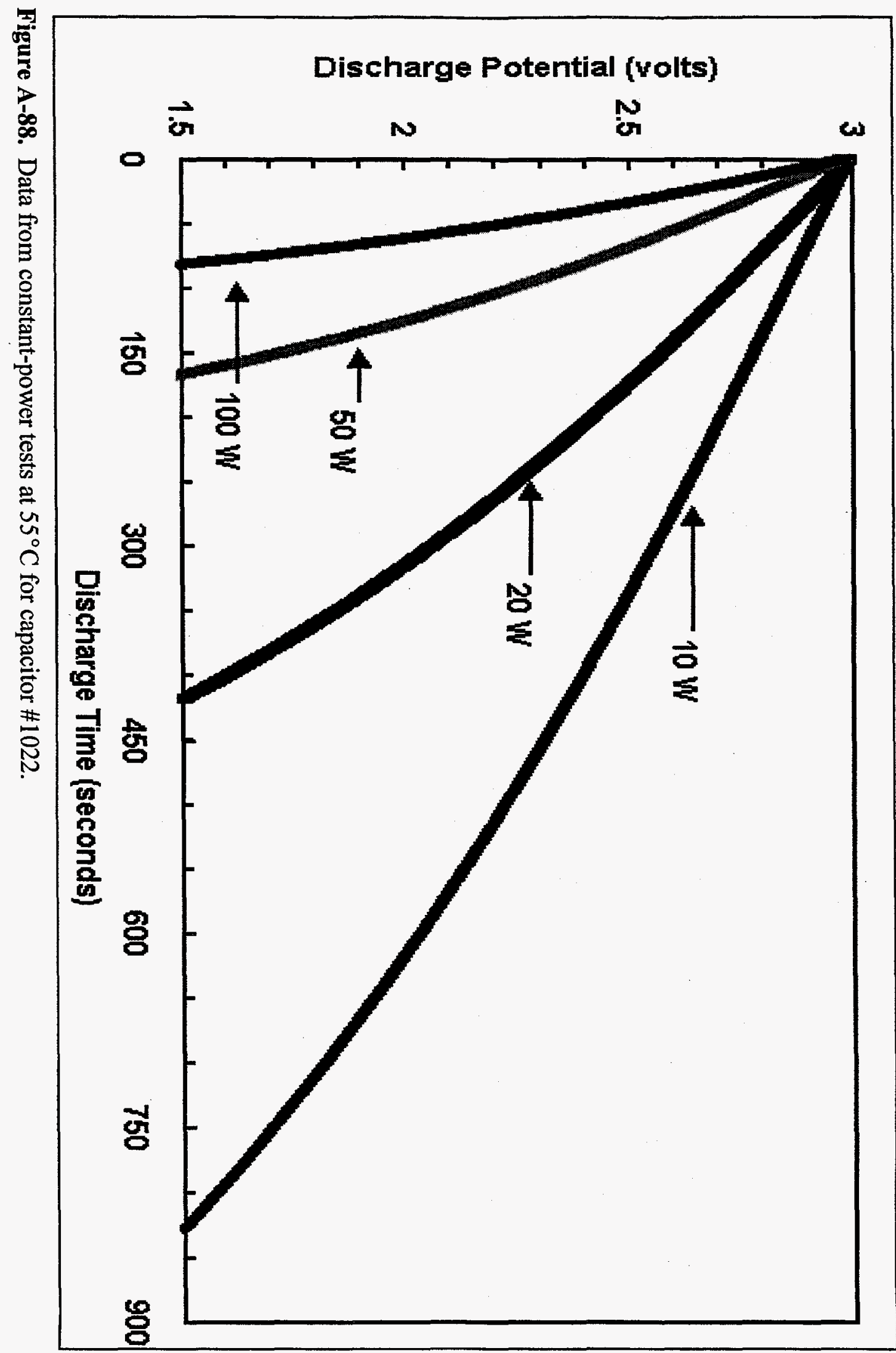




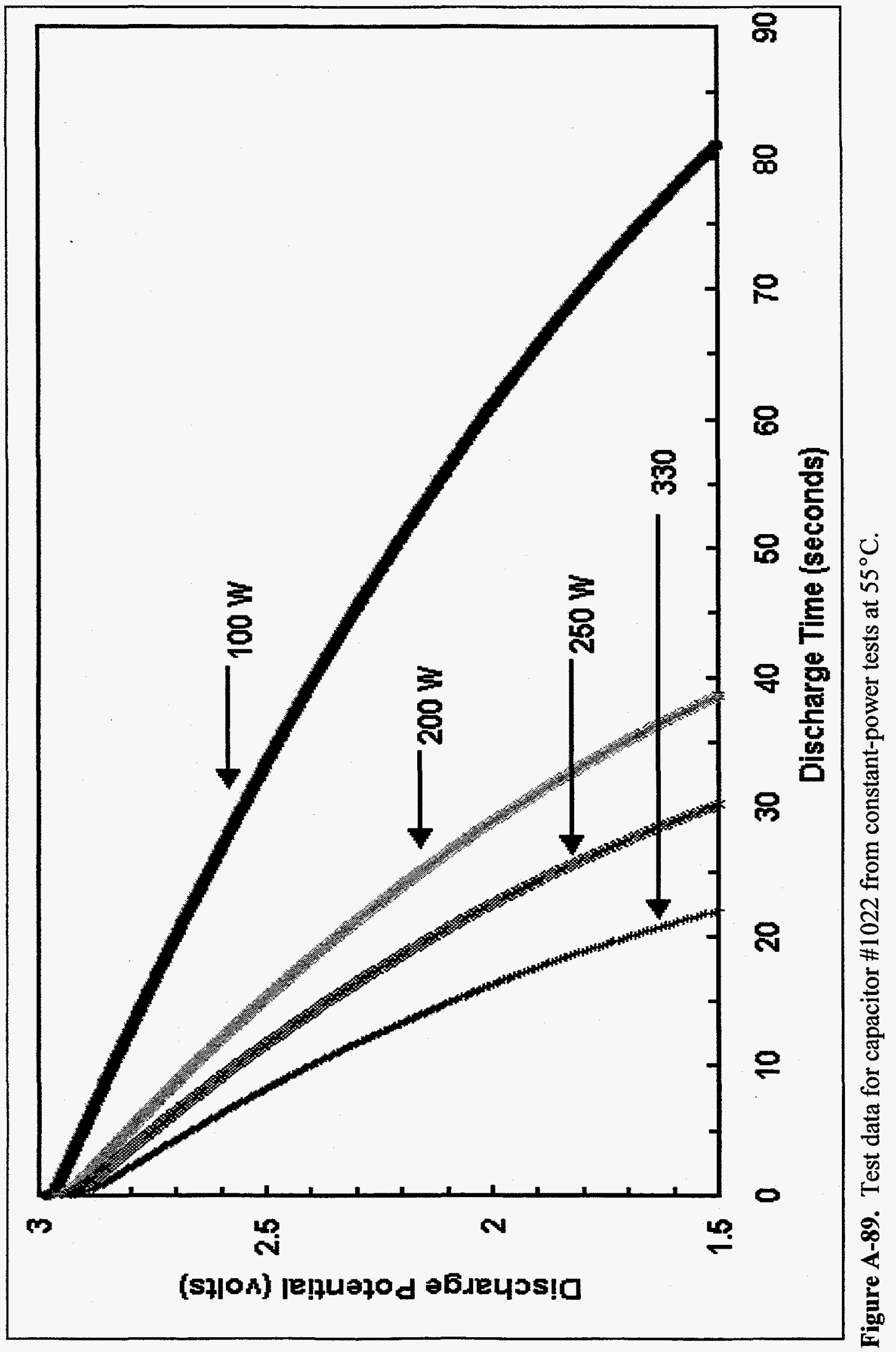




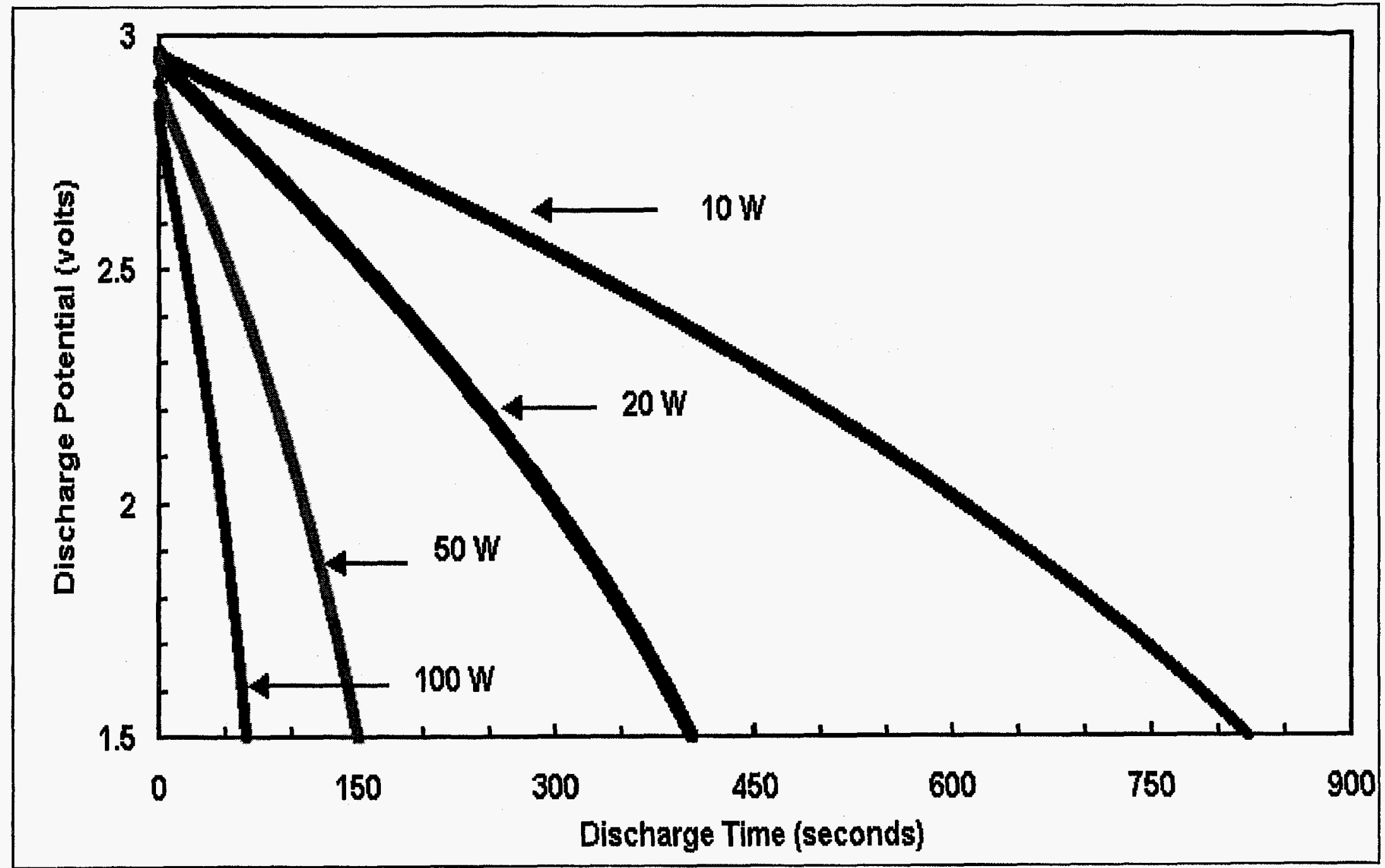

Figure A-90. Test data from constant-power tests at $-20^{\circ} \mathrm{C}$ for capacitor $\# 1022$. 


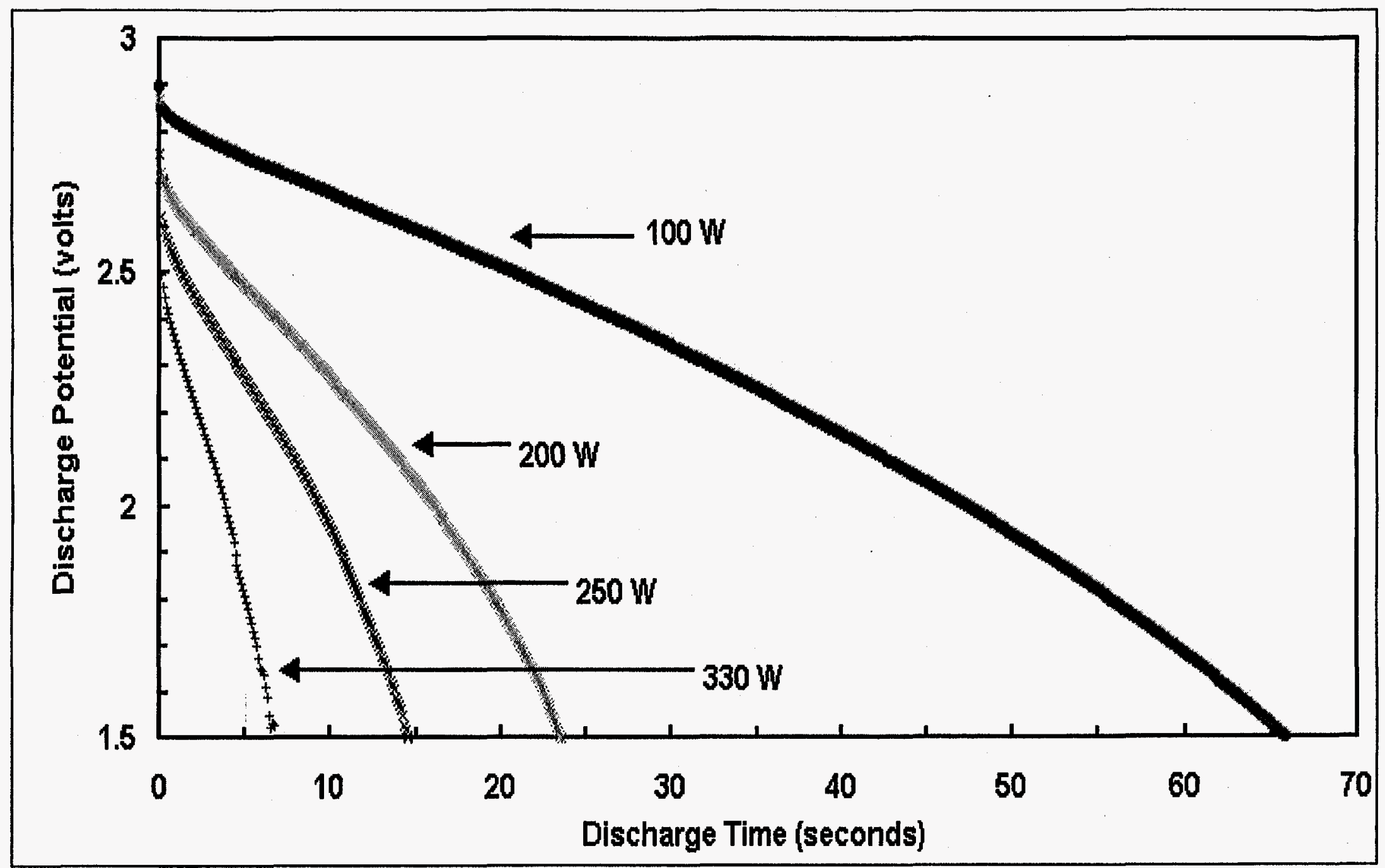

Figure A-91. Constant-power test data for capacitor $\# 1022$ at $-20^{\circ} \mathrm{C}$. 


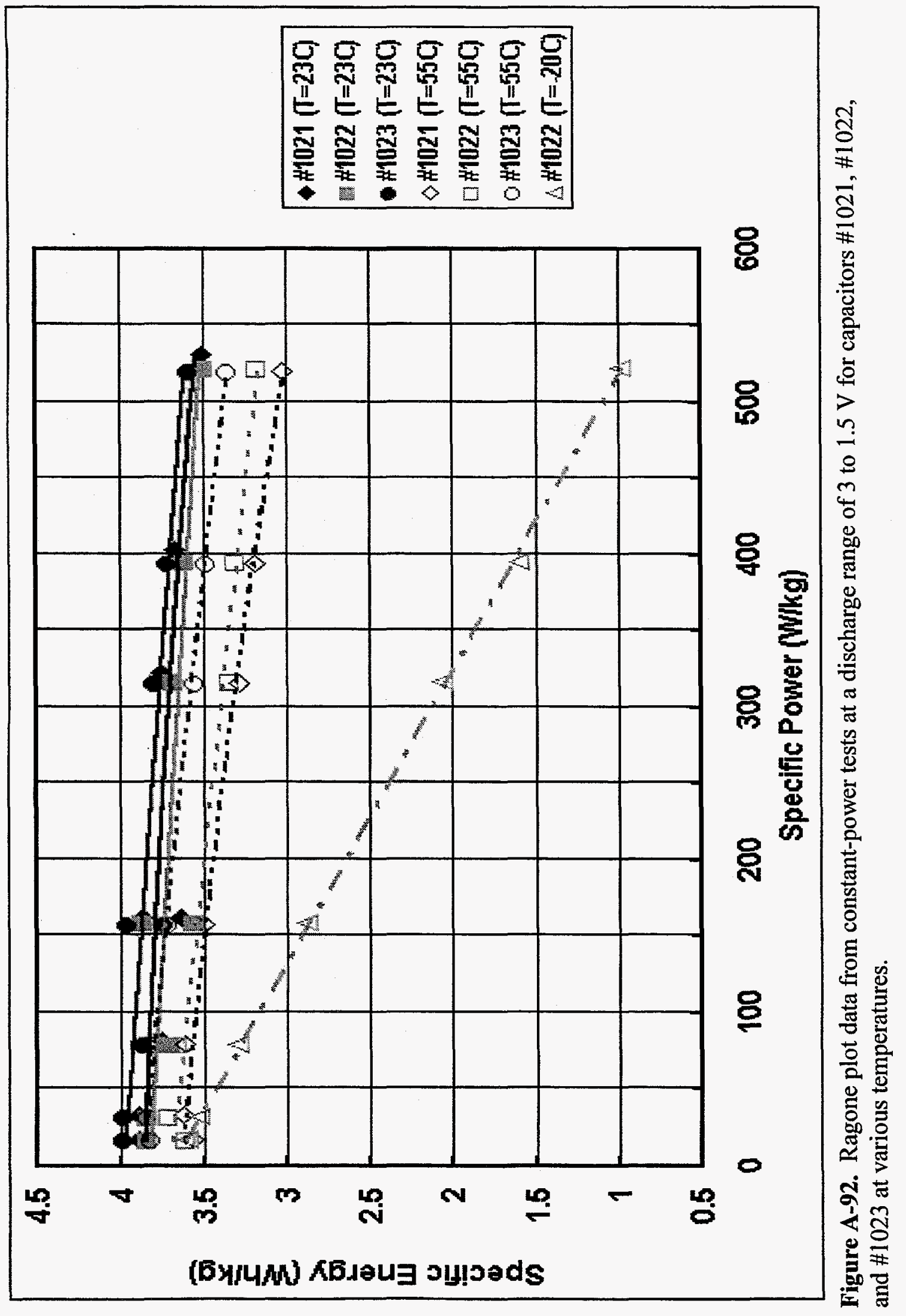




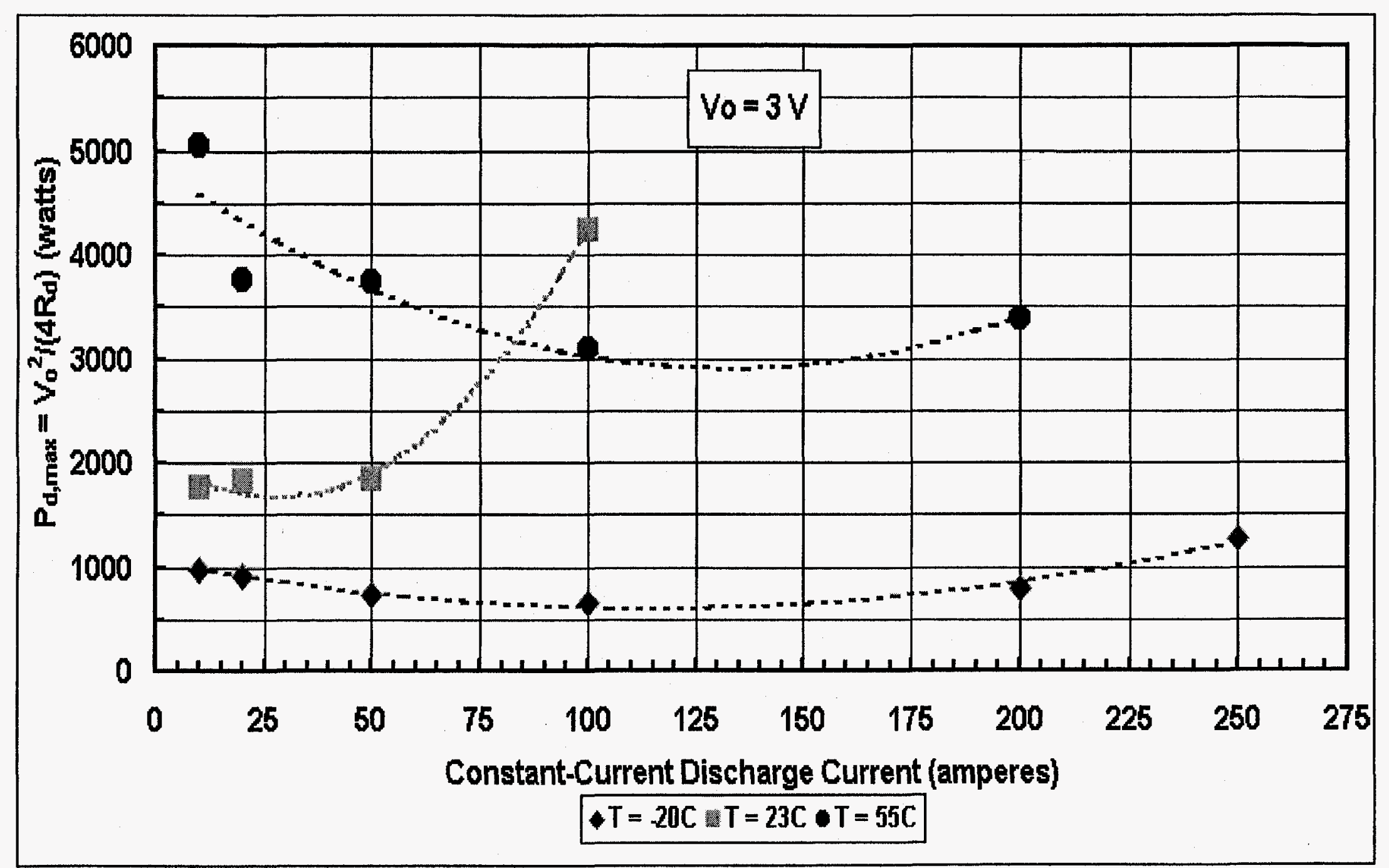

Figure A-93. Calculated $\mathrm{P}_{\mathrm{d}, \max }$ for capacitor \#1022; test data from constant-current at a range of 0.05 to $3 \mathrm{~V}$ at $-20^{\circ} \mathrm{C}, 0.01$ to $3 \mathrm{~V}$ at $23^{\circ} \mathrm{C}$, and 1.5 to $3 \mathrm{~V}$ at $55^{\circ} \mathrm{C}$. 


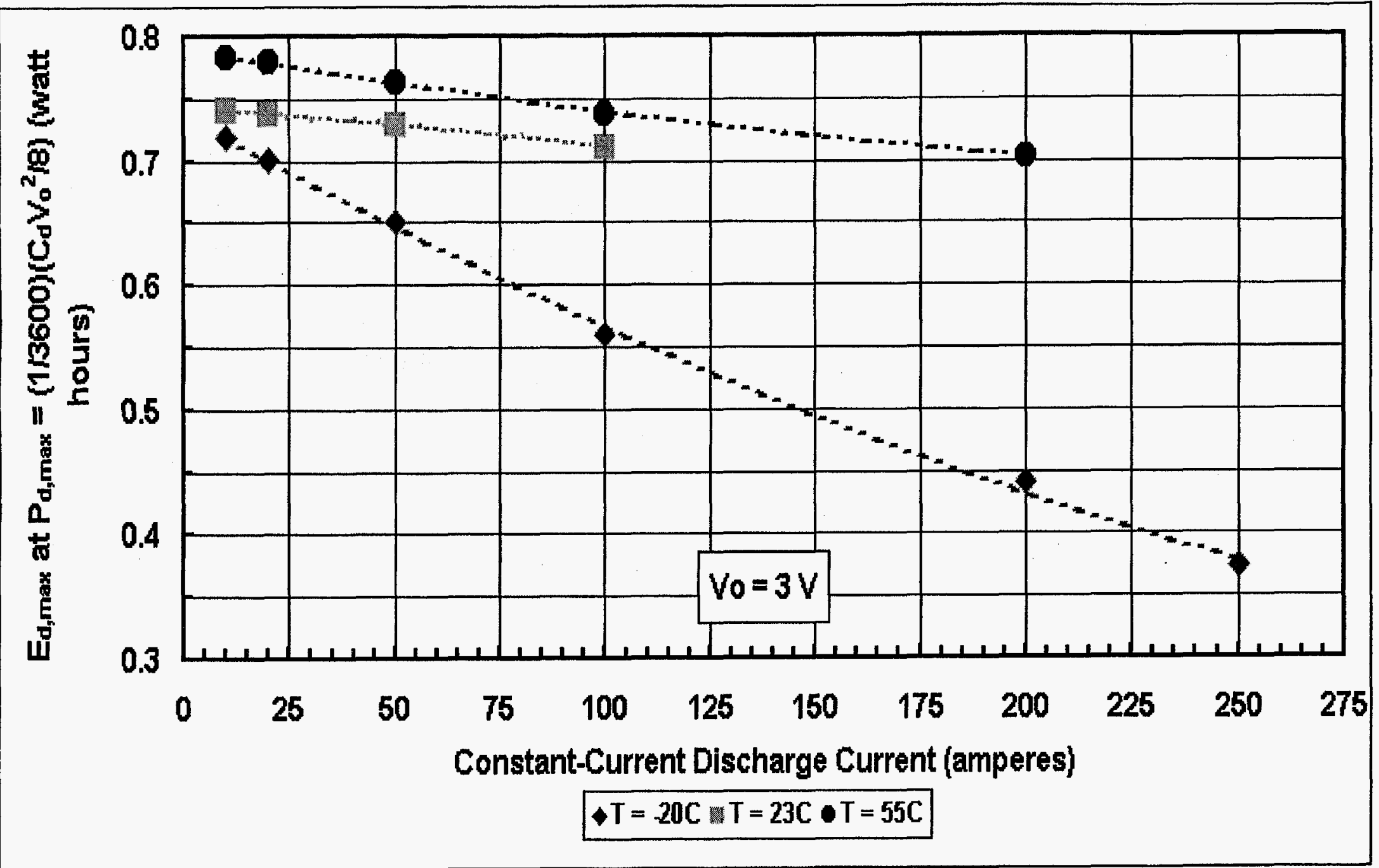

Figure A-94. Calculated $\mathrm{E}_{\mathrm{d}, \max }$ at $\mathrm{P}_{\mathrm{d}, \max }$ test data from constant-current for 0.05 to $3 \mathrm{~V}$ at $-20^{\circ} \mathrm{C}, 0.01$ to $3 \mathrm{~V}$ for $23^{\circ} \mathrm{C}$, and 1.5 to $3 \mathrm{~V}$ at $55^{\circ} \mathrm{C}$ for capacitor $\# 1022$. 


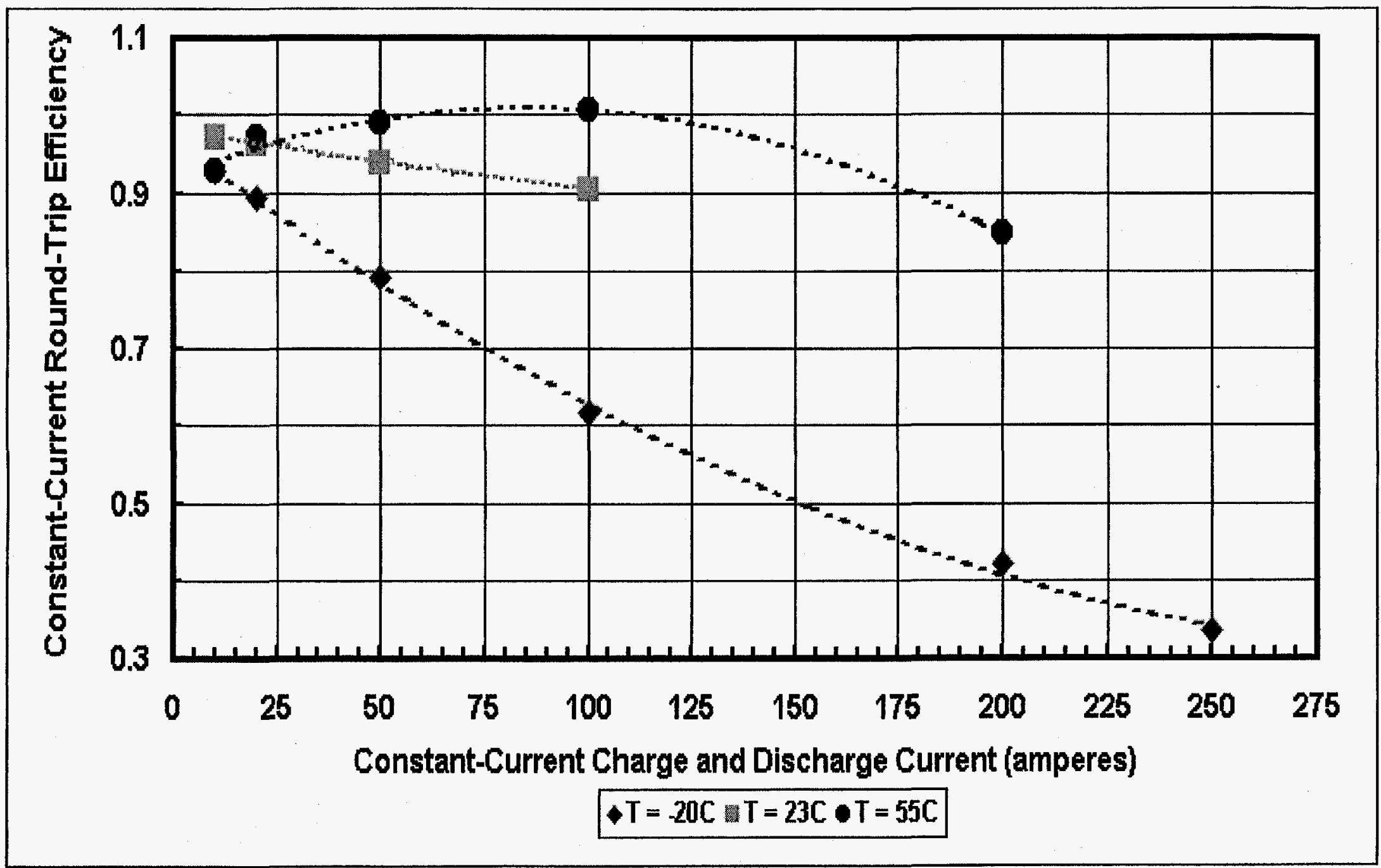

Figure A-95. Constant-current round-trip efficiency values data for capacitor \#1022 from constant-current tests for 0.05 to $3 \mathrm{~V}$ at $-20^{\circ} \mathrm{C}, 0.01 \mathrm{~V}$ to $3 \mathrm{~V}$ at $23^{\circ} \mathrm{C}$, and 1.5 to $3 \mathrm{~V}$ at $55^{\circ} \mathrm{C}$. 


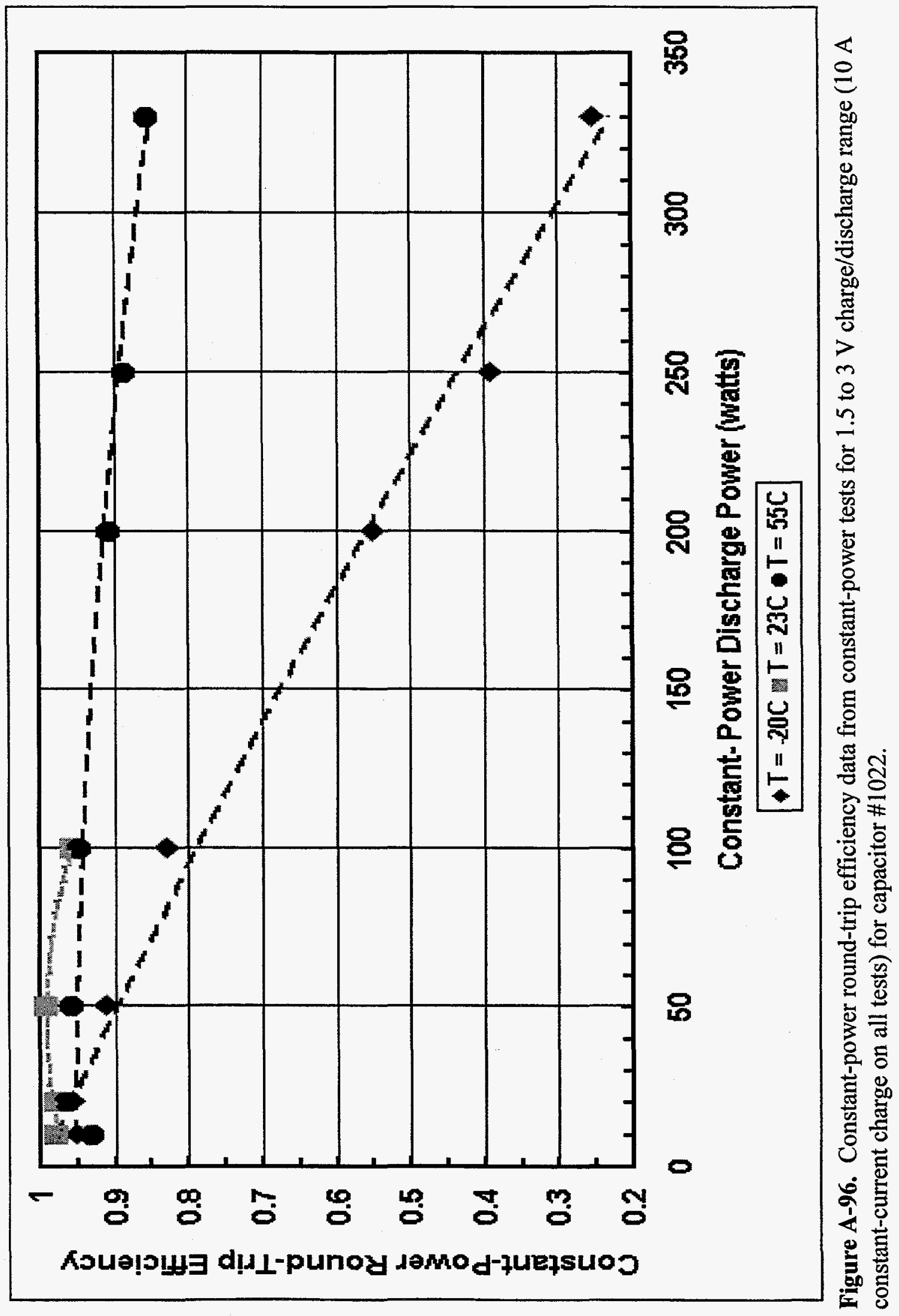




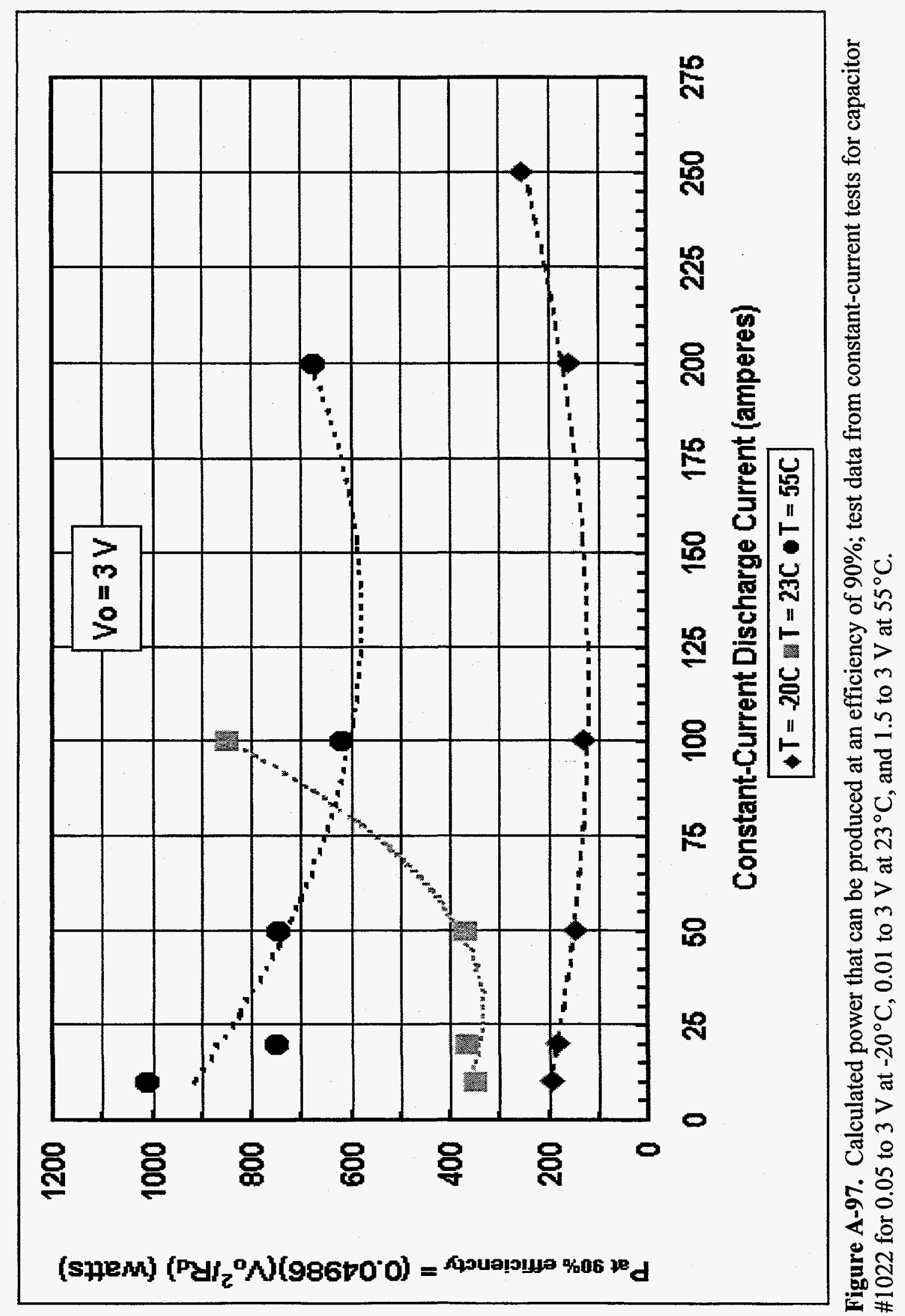




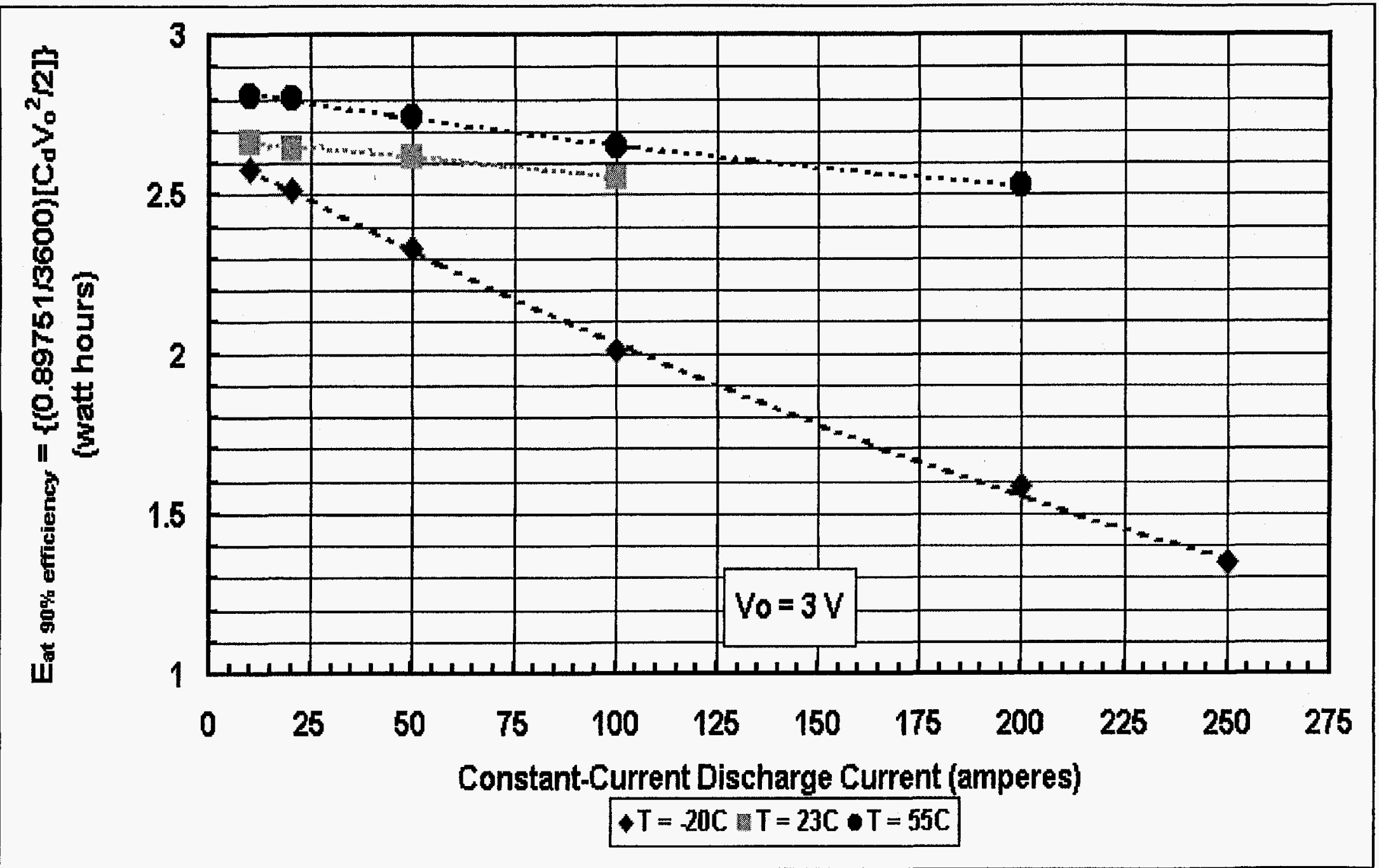

Figure A-98. Calculated available energy at an efficiency of $90 \%$; constant-current test data for 0.05 to $3 \mathrm{~V}$ at $-20^{\circ} \mathrm{C}, 0.01$ to $3 \mathrm{~V}$ at $23^{\circ} \mathrm{C}$, and 1.5 to $3 \mathrm{~V}$ at $55^{\circ} \mathrm{C}$ for capacitor $\# 1022$. 


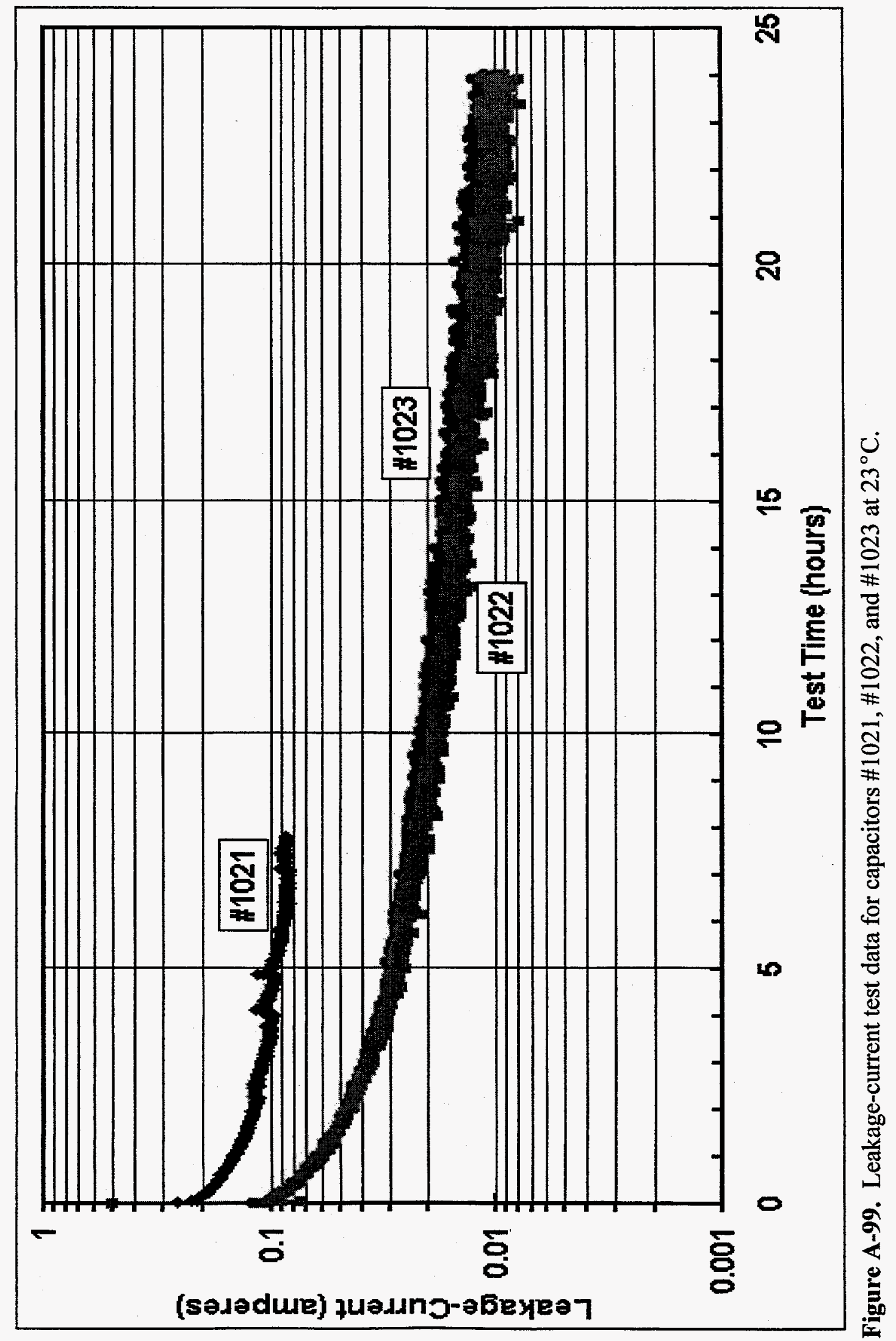




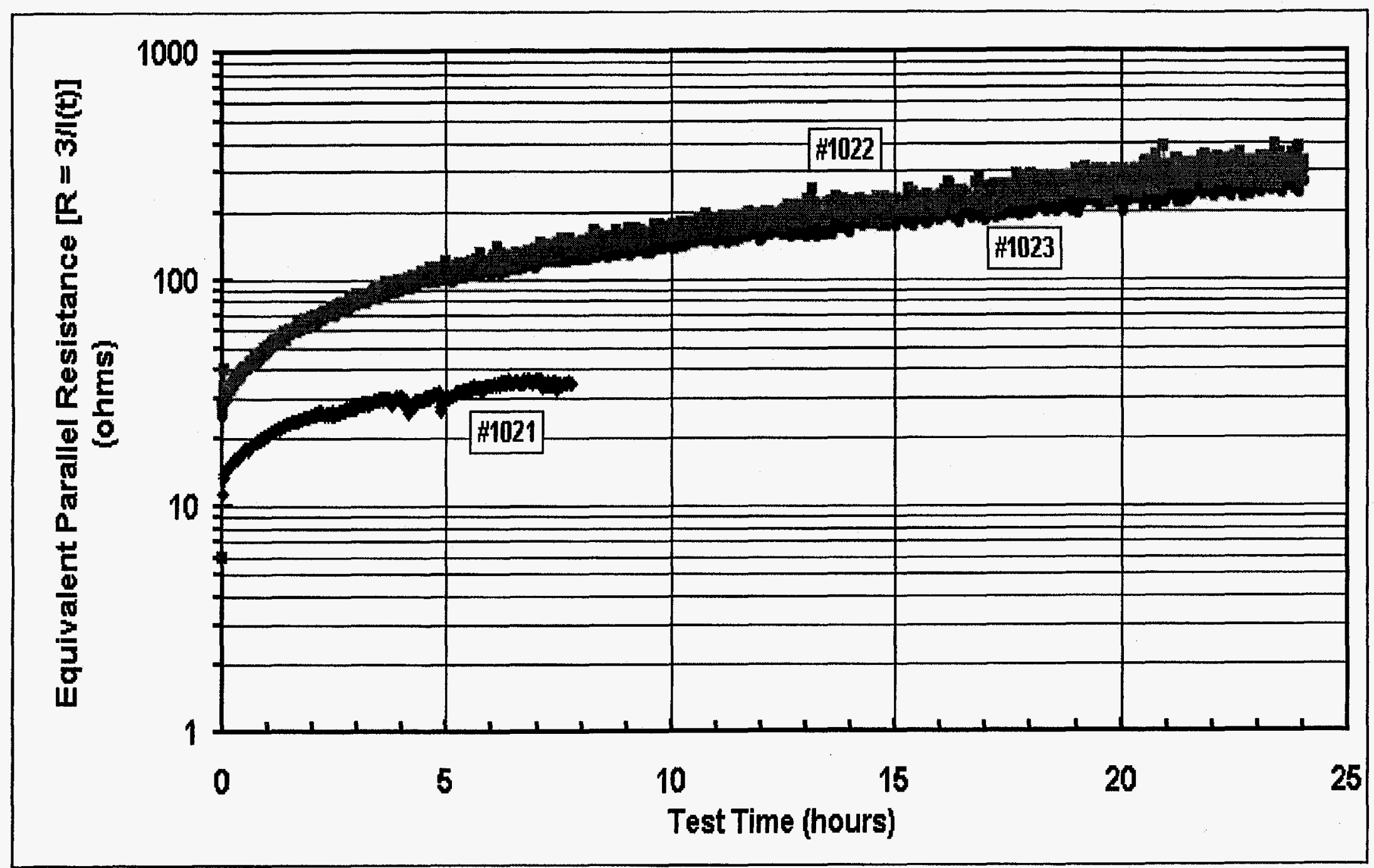

Figure A-100. Equivalent parallel resistance for capacitors $\# 1021, \# 1022$, and $\# 1023$ at $23^{\circ} \mathrm{C}$ calculated from the leakage-current test data. 


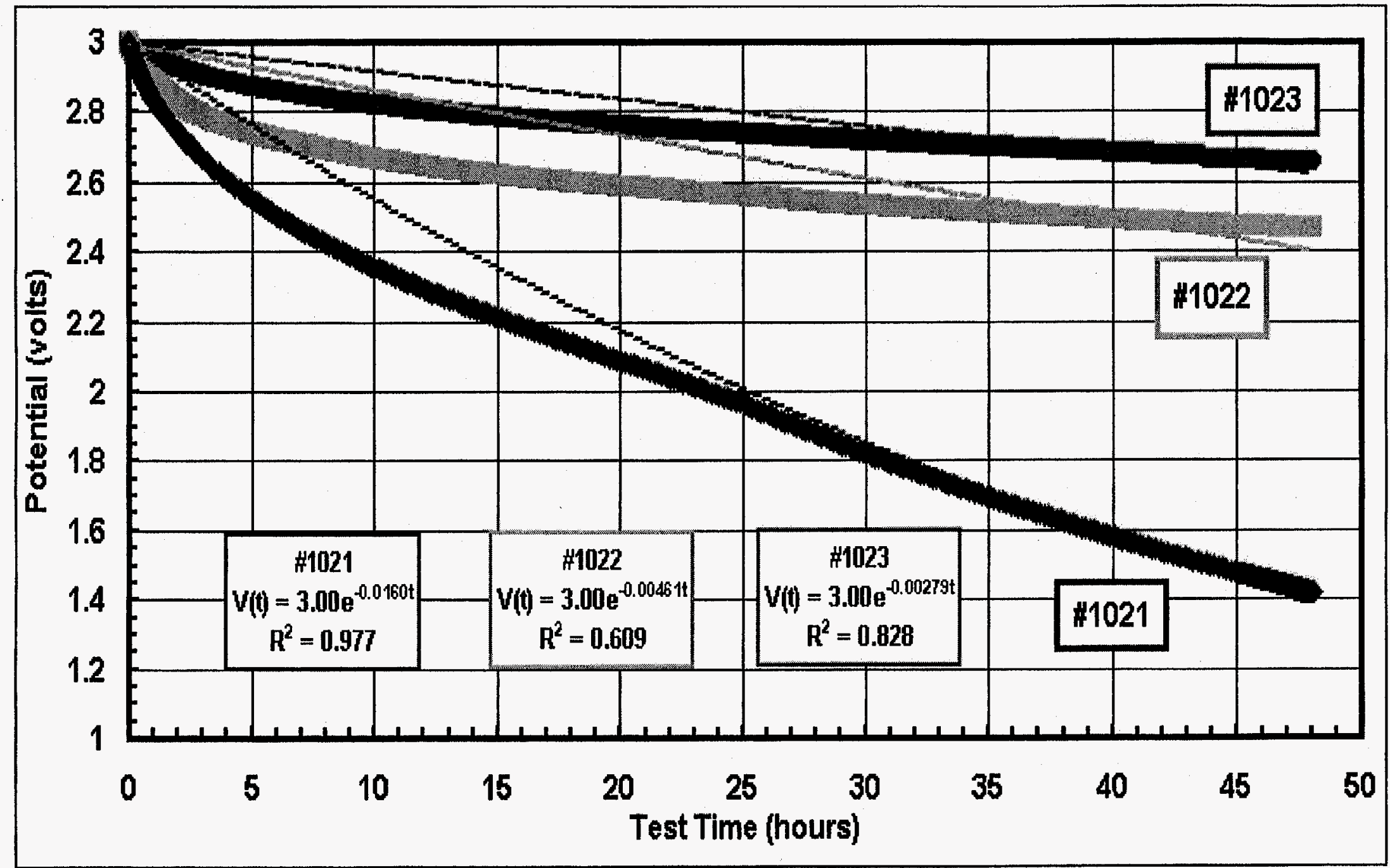

Figure A-101. Data from self-discharge test at $23^{\circ} \mathrm{C}$ for capacitors $\# 1021, \# 1022$, and \#1023 and fits to an exponential time dependence model. 


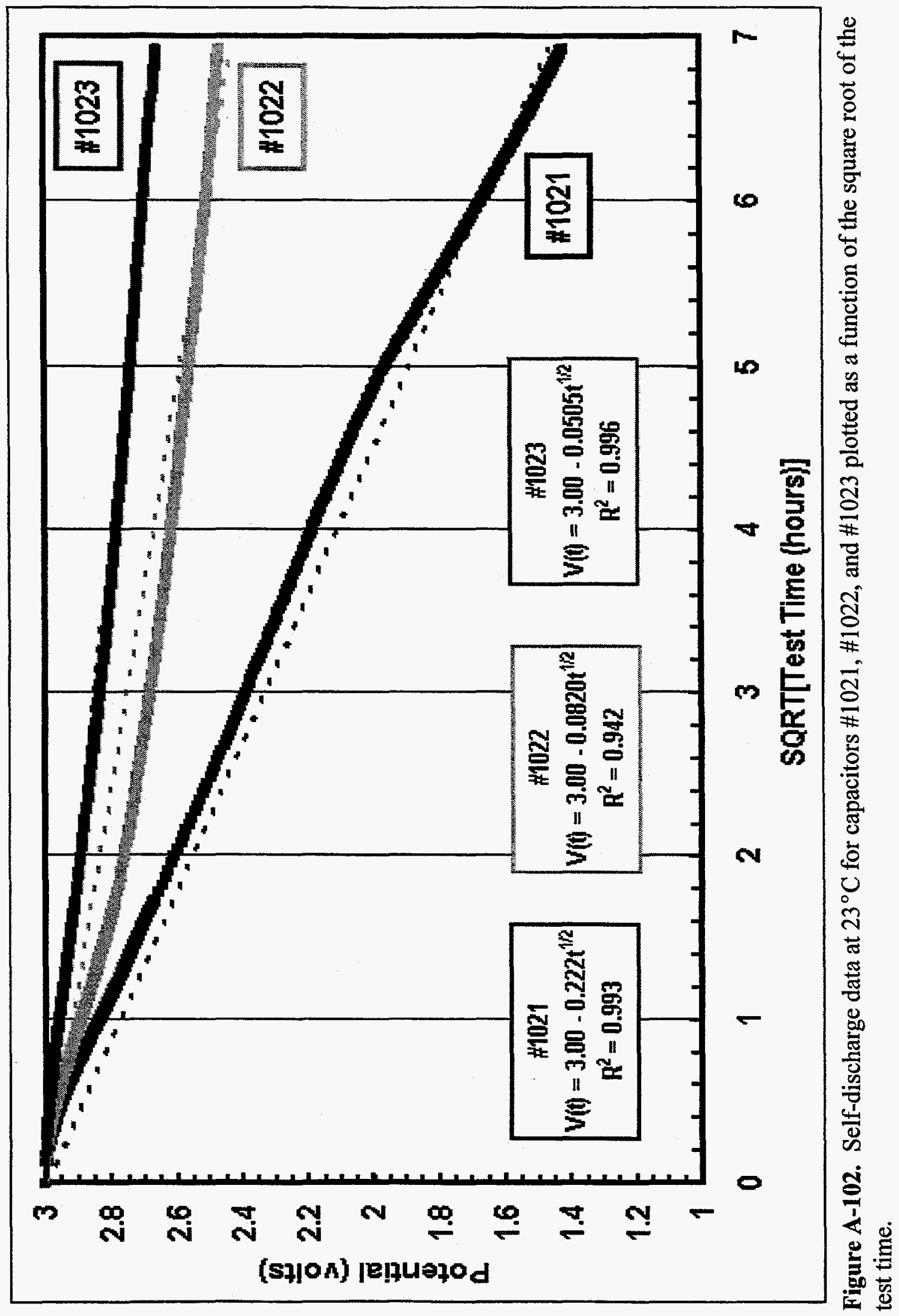




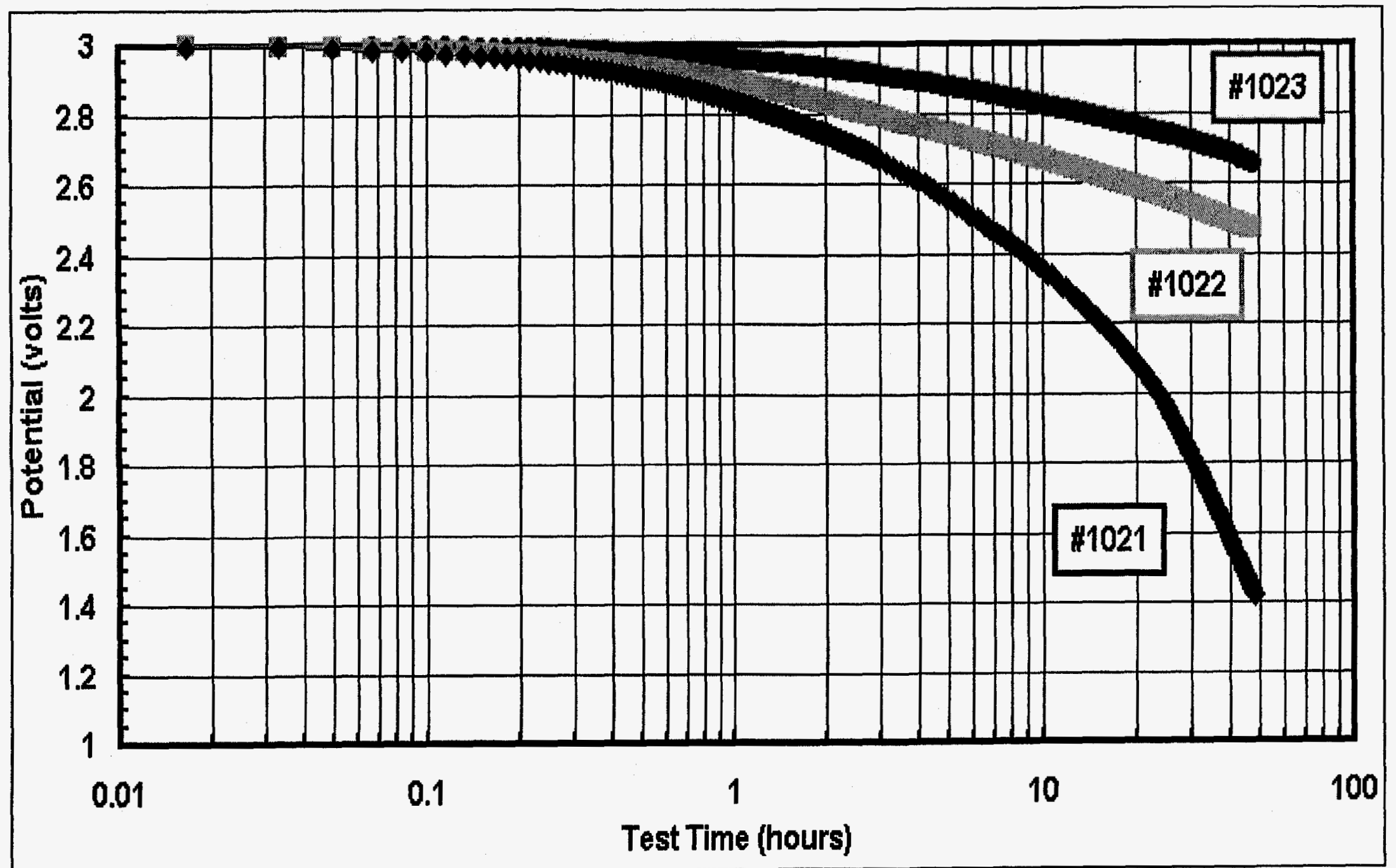

Figure A-103. Self-discharge test data at $23^{\circ} \mathrm{C}$ for capacitors $\# 1021, \# 1022$, and \#1023 plotted as a function of the log ${ }_{10}$ of the test time. 


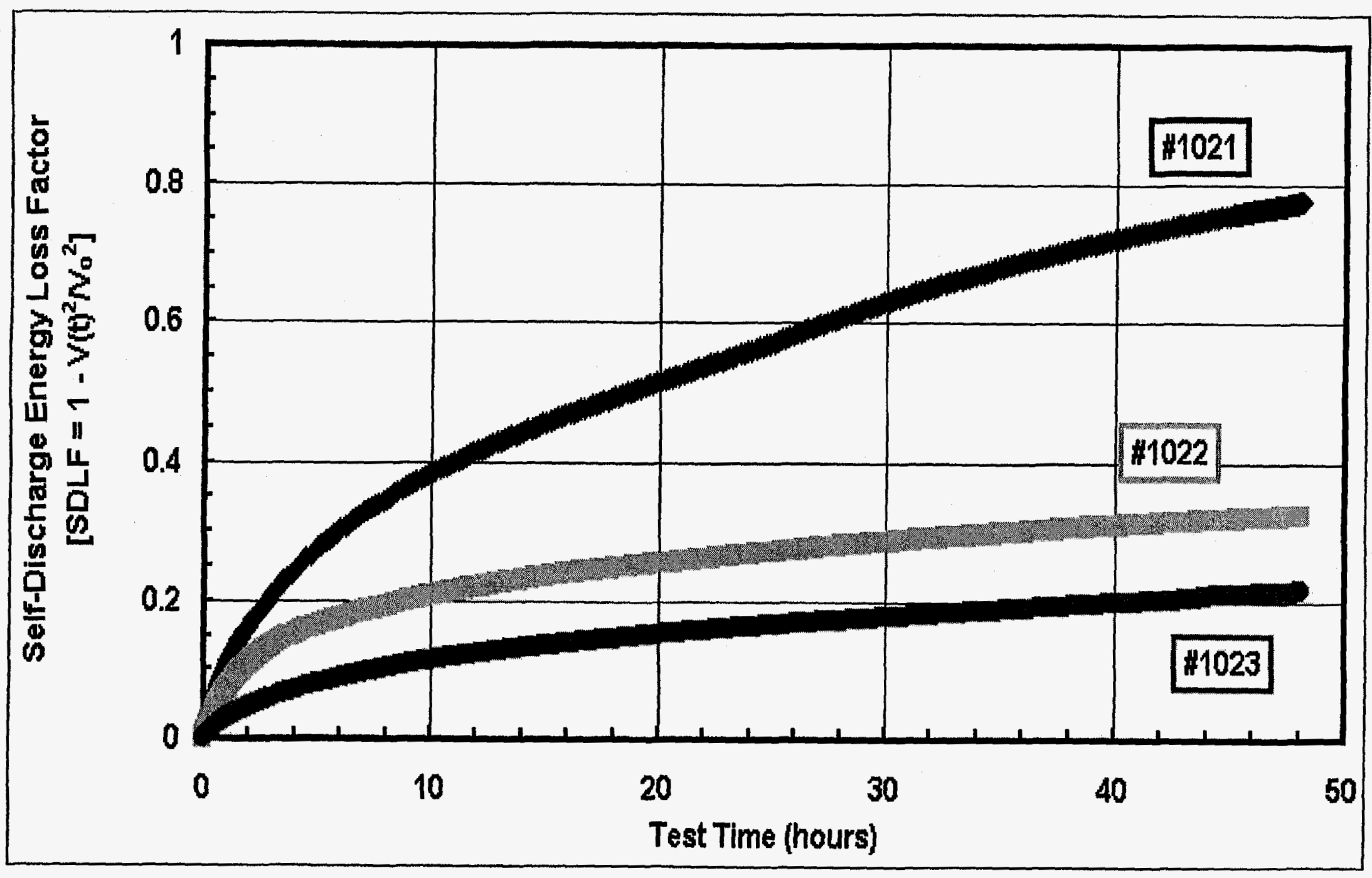

Figure A-104. Self-discharge energy loss factor for capacitors $\# 1021, \# 1022$, and $\# 1023$ at $23^{\circ} \mathrm{C}$. 


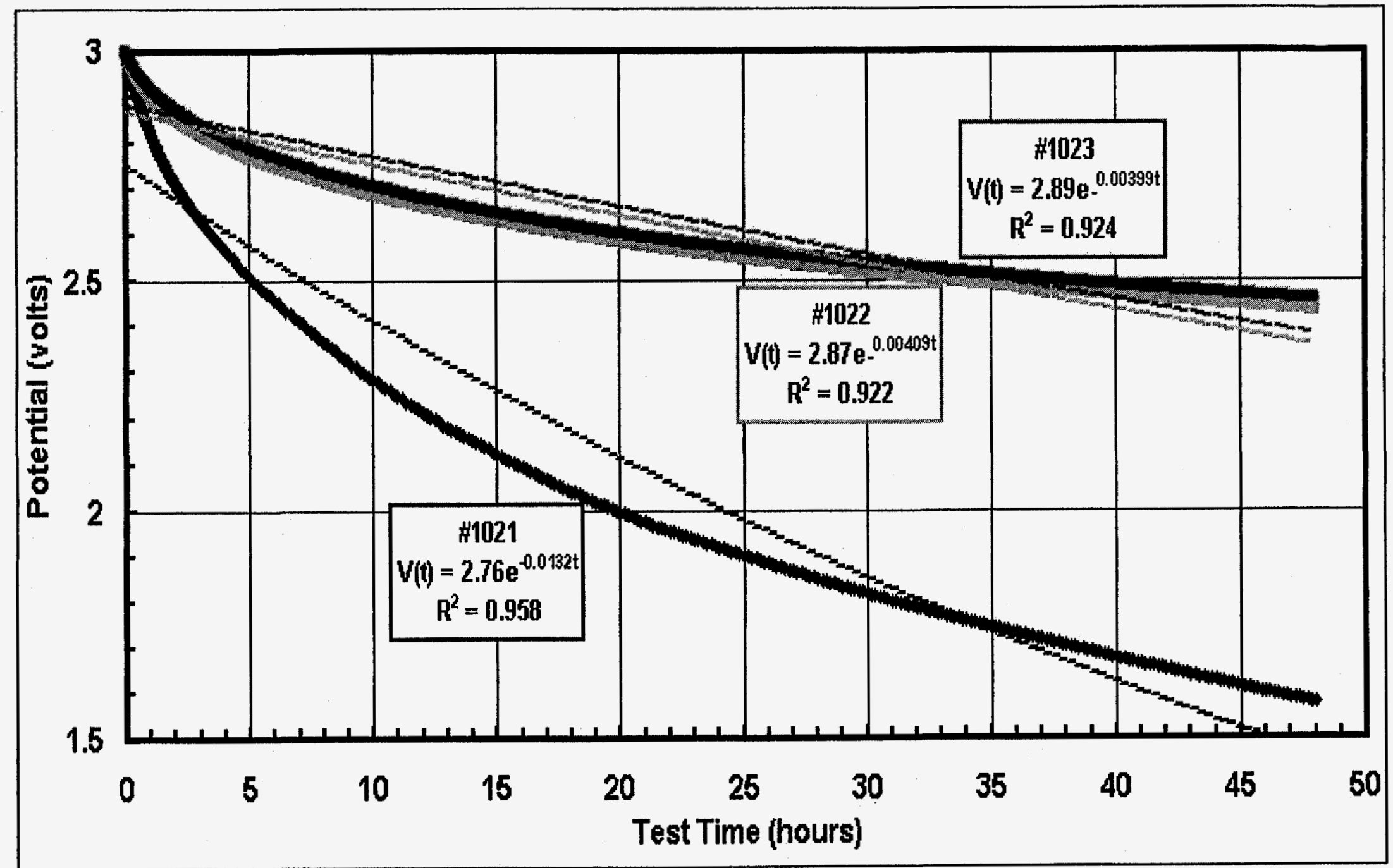

Figure A-105. Data from self-discharge test at $55^{\circ} \mathrm{C}$ for capacitors \#1021, \#1022, and \#1023 and fits to an exponential time dependence model. 


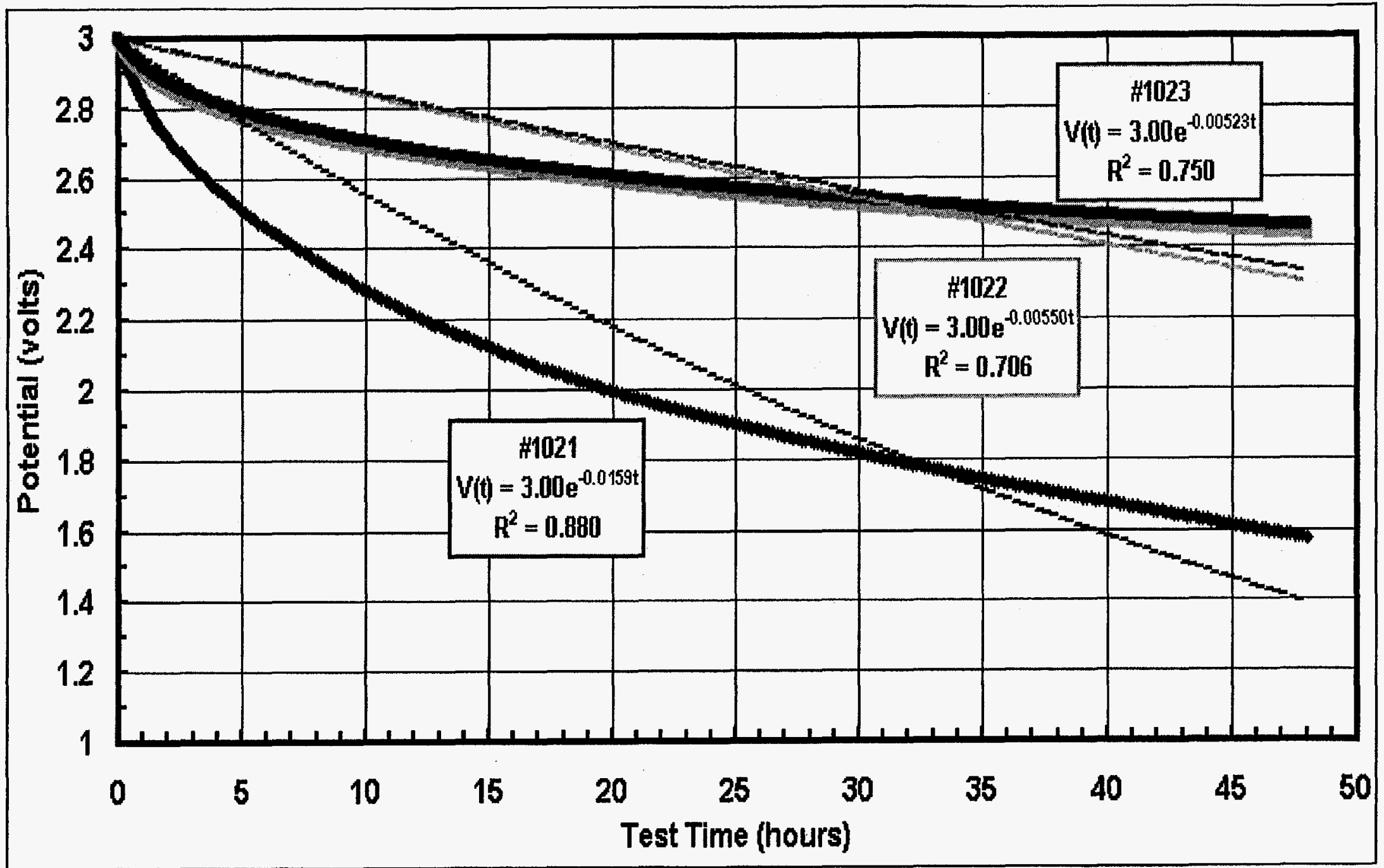

Figure A-106. Self-discharge test data at $55^{\circ} \mathrm{C}$ for capacitors $\# 1021, \# 1022$, and $\# 1023$ and fits to an exponential time dependence model. 


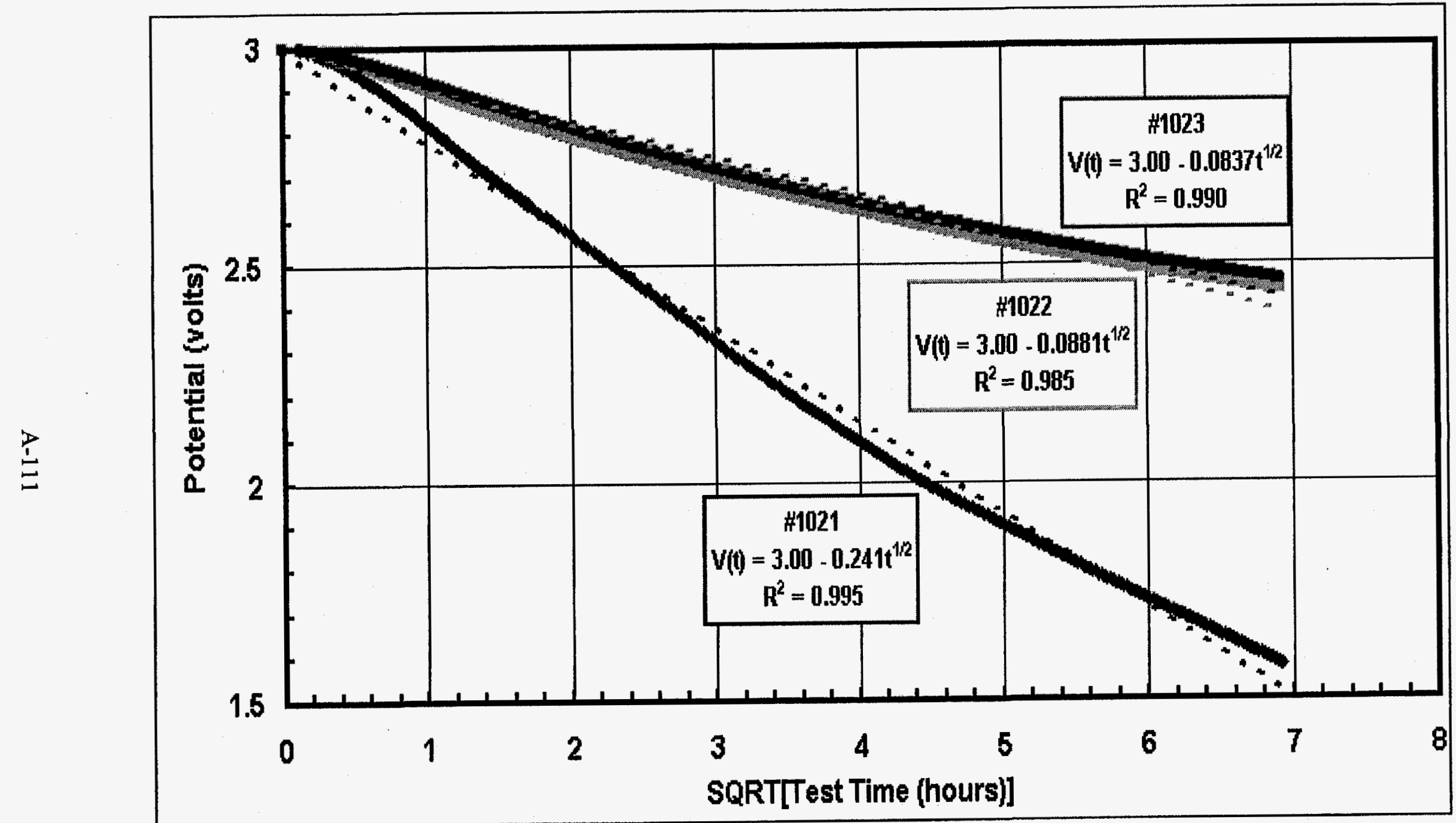

Figure A-107. Data from self-discharge test at $55^{\circ} \mathrm{C}$ for capacitors $\# 1021$, \#1022, and \#1023 and fits to a square-root of time dependence model. 


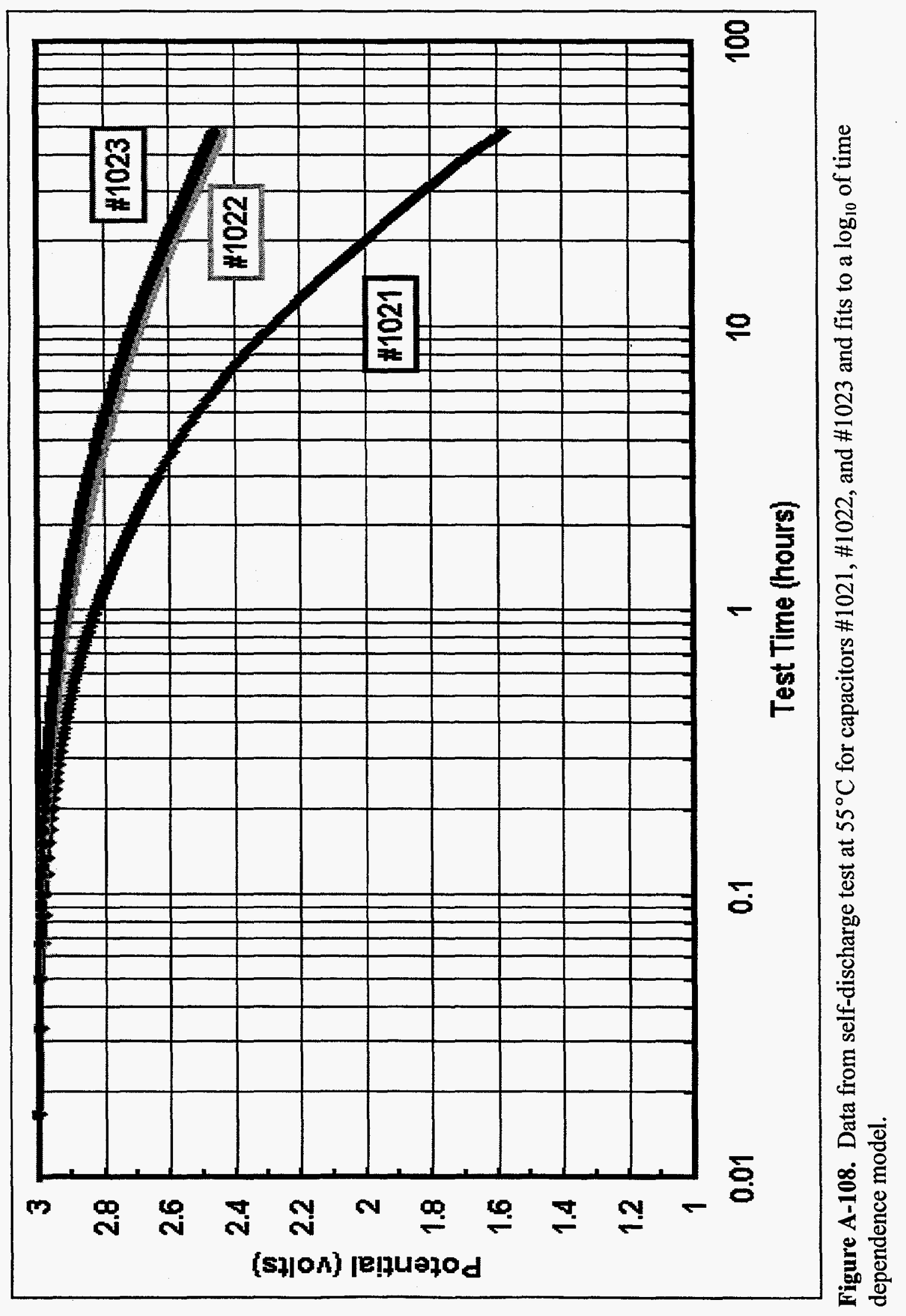




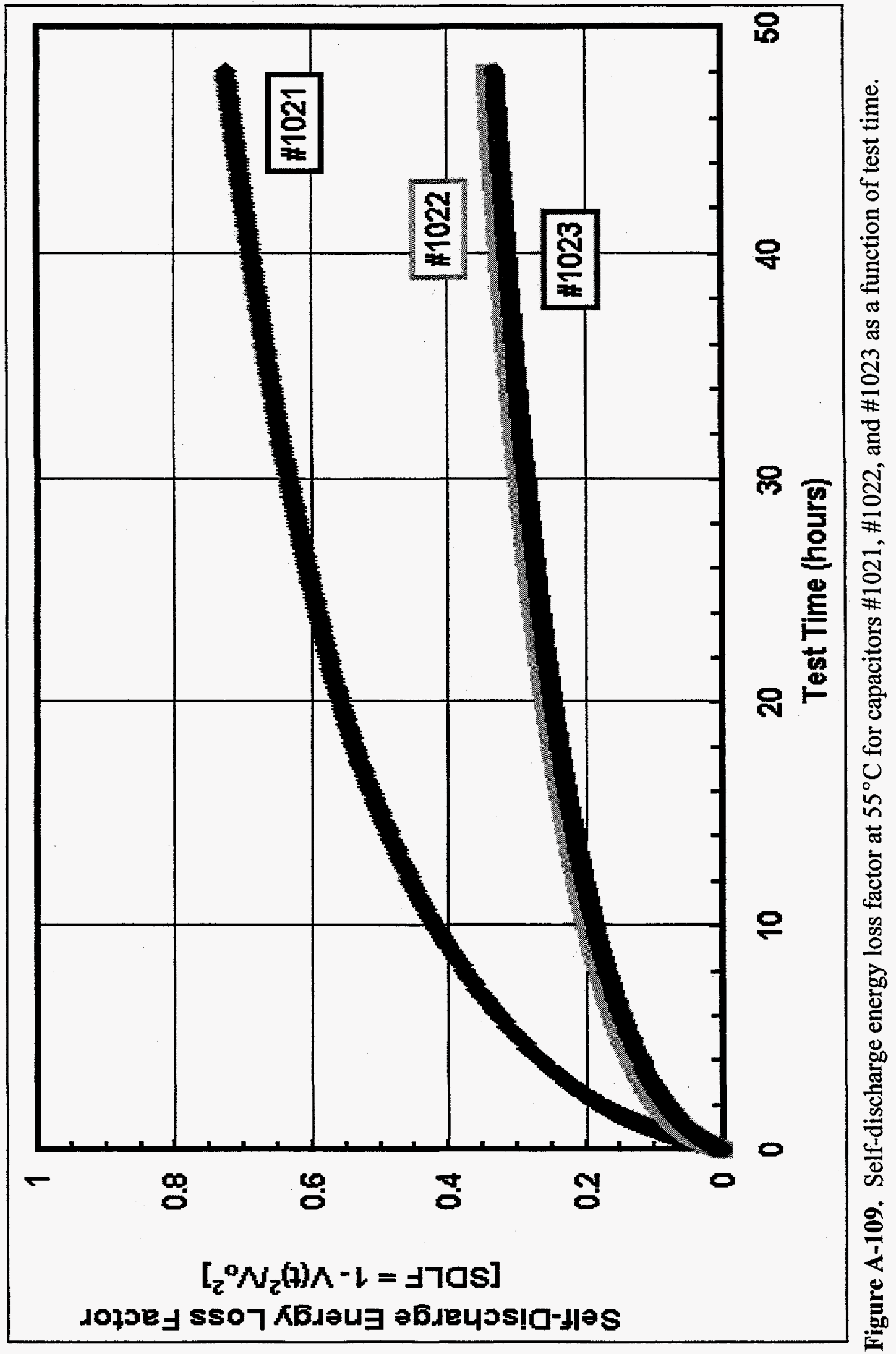




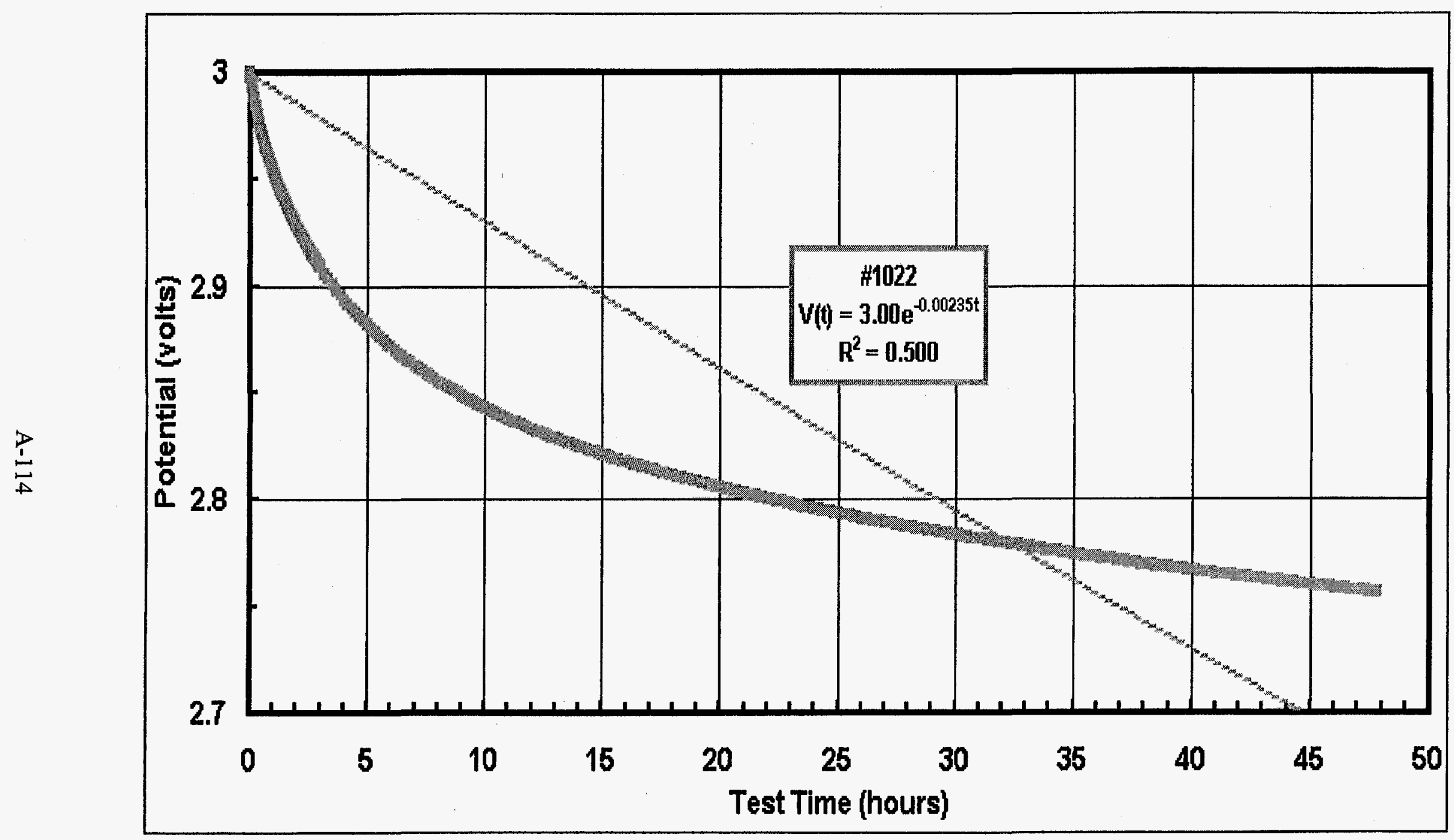

Figure A-110. Self-discharge test data at $-20^{\circ} \mathrm{C}$ for capacitor \#1022. A fit to an exponential time dependence is shown. 


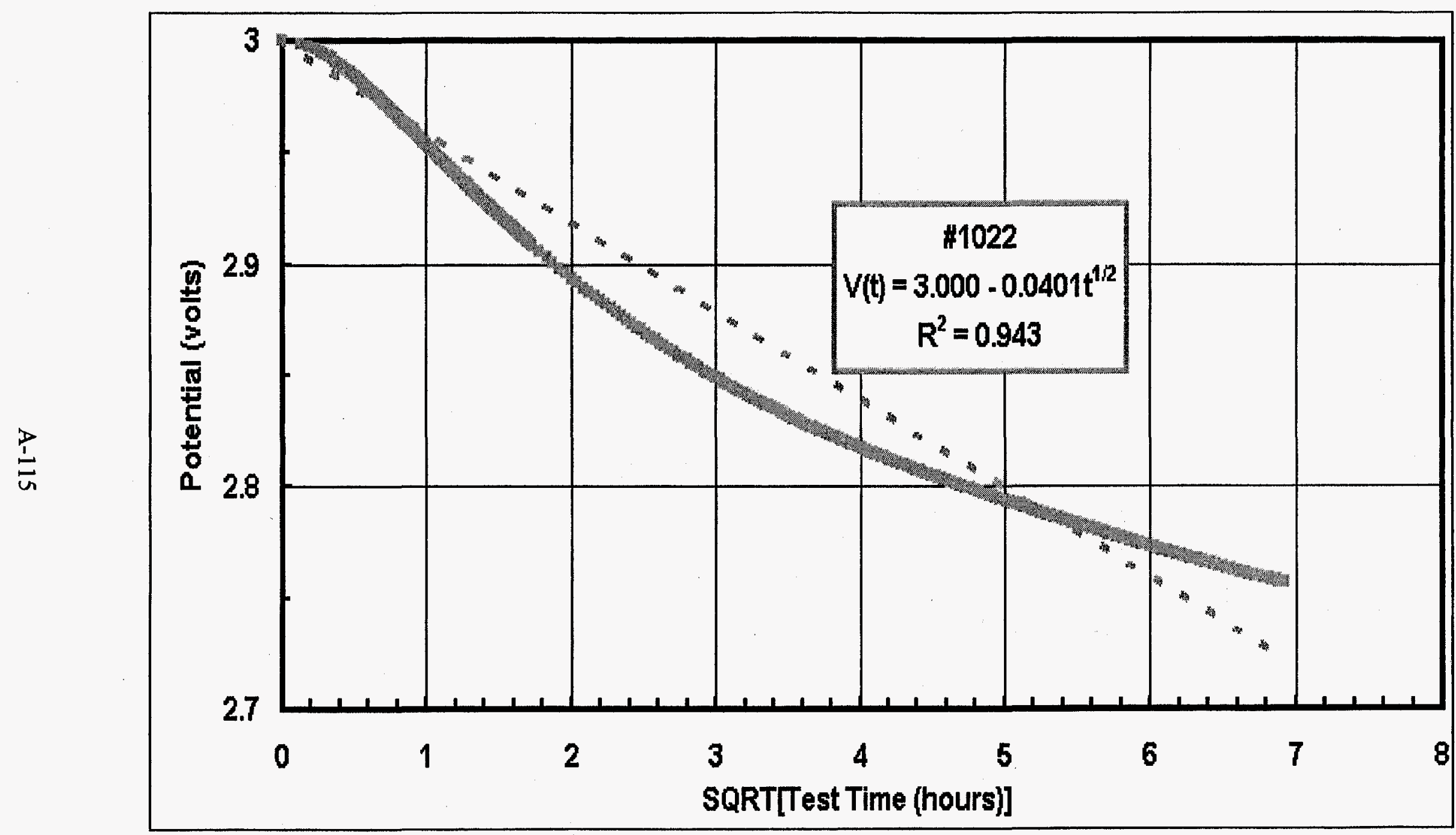

Figure A-111. Test data from self-discharge test for capacitor $\# 1022$ at $-20^{\circ} \mathrm{C}$. A fit to a square-root time dependence is shown. 


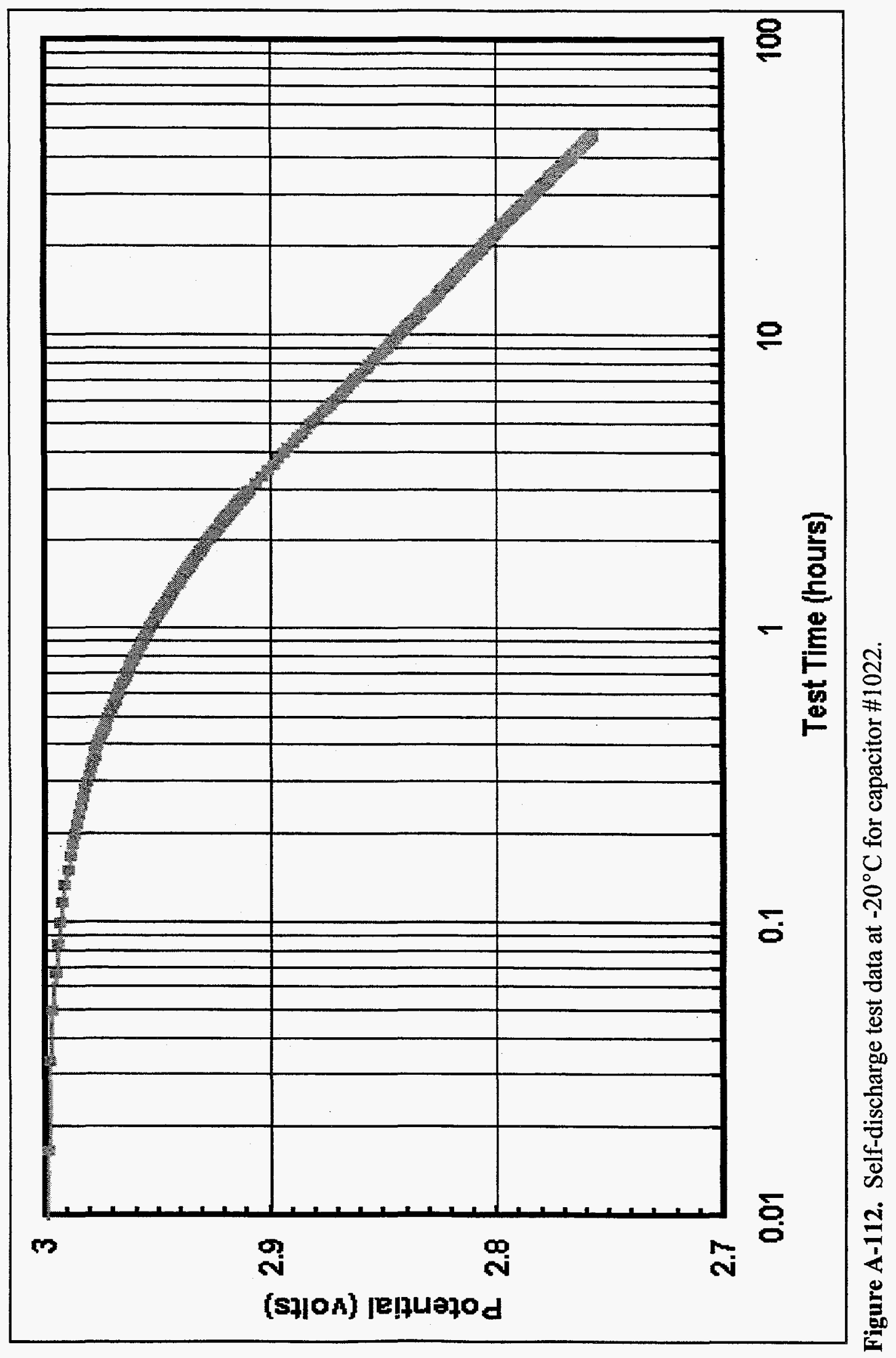




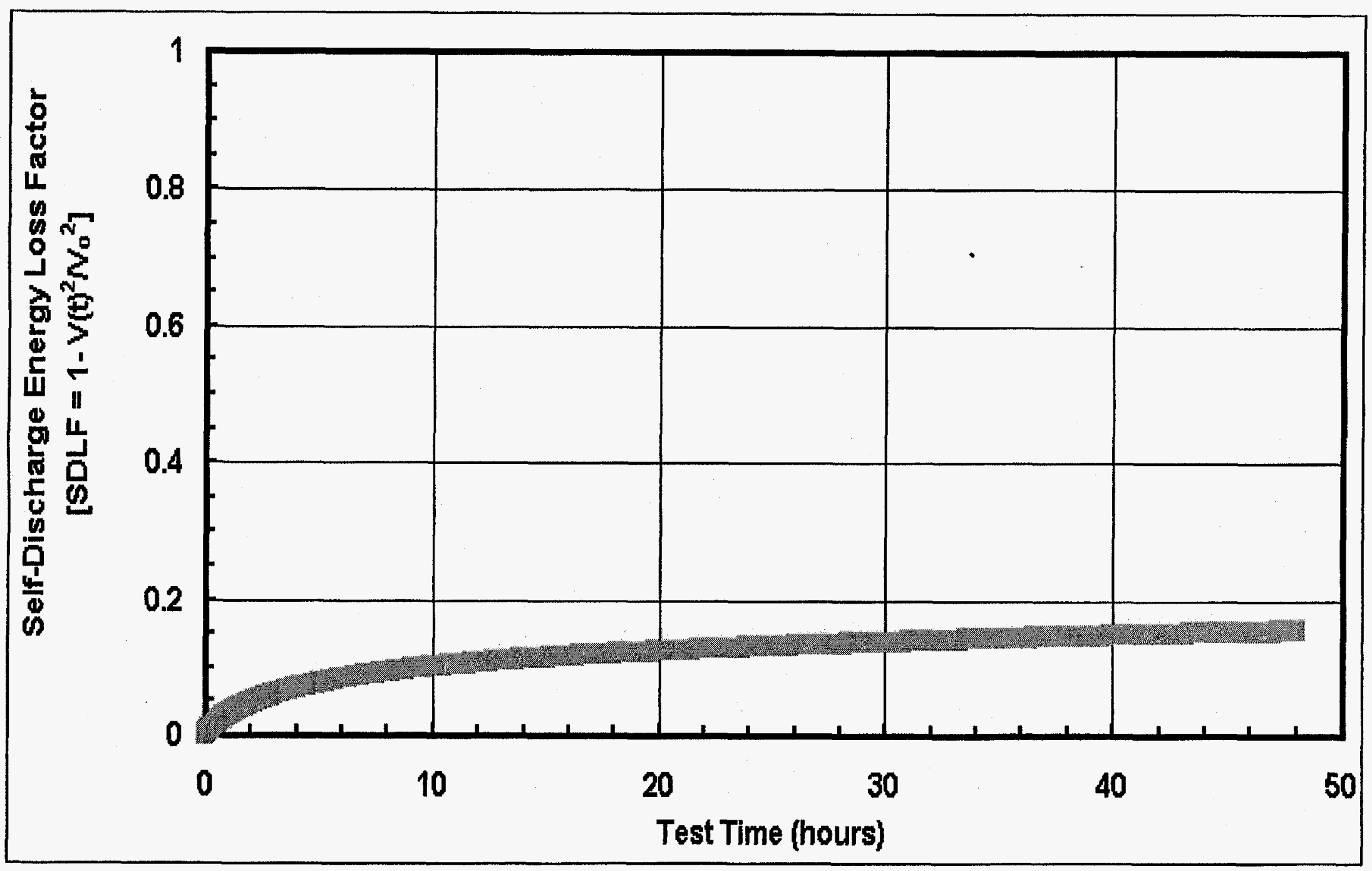

Figure A-113. Test data from self-discharge test for capacitor $\# 1022$ at $-20^{\circ} \mathrm{C}$. 


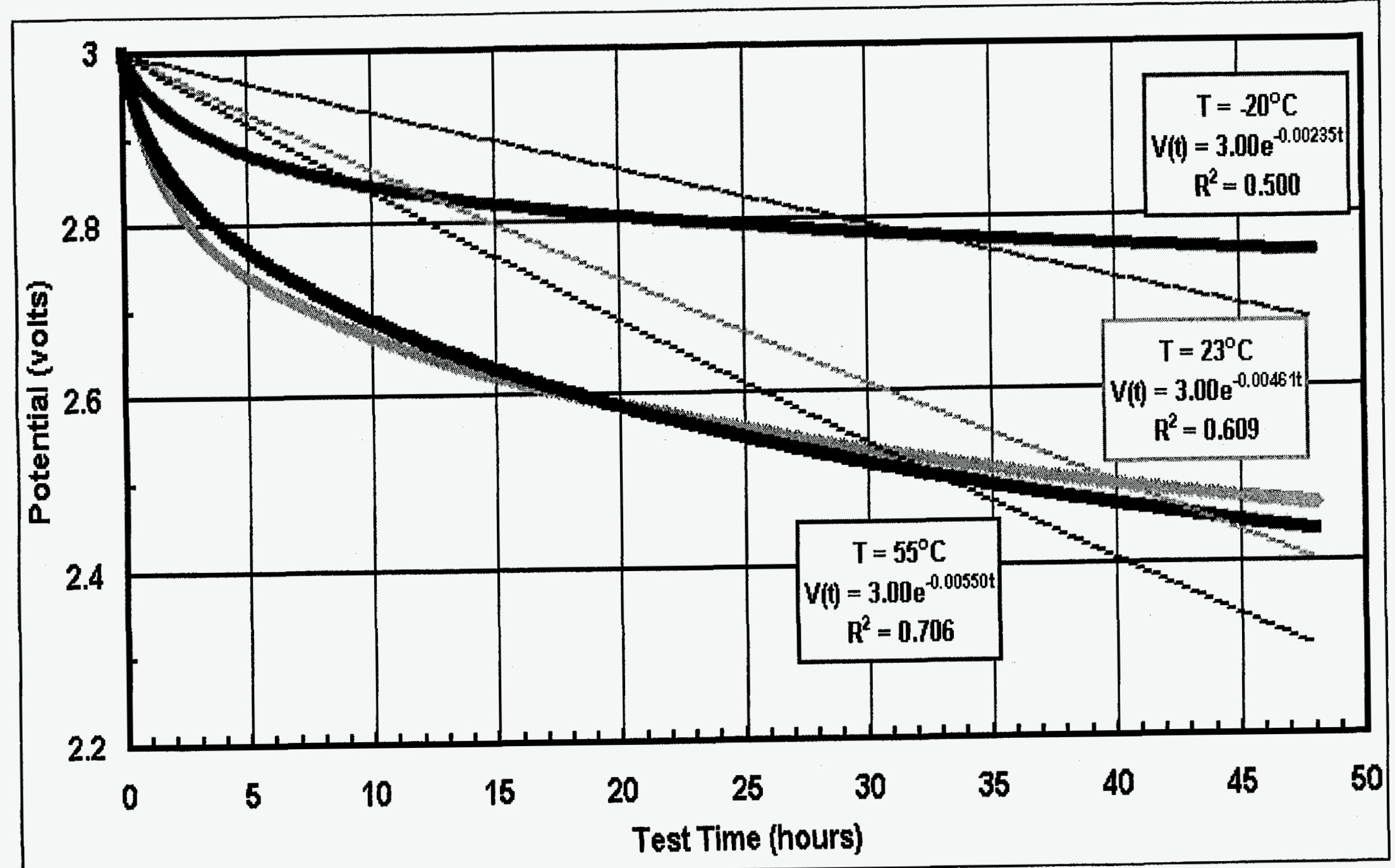

Figure A-114. Data from self-discharge tests for capacitor \#1022 as a function of temperature. Exponential time fits to the voltage data are shown. 


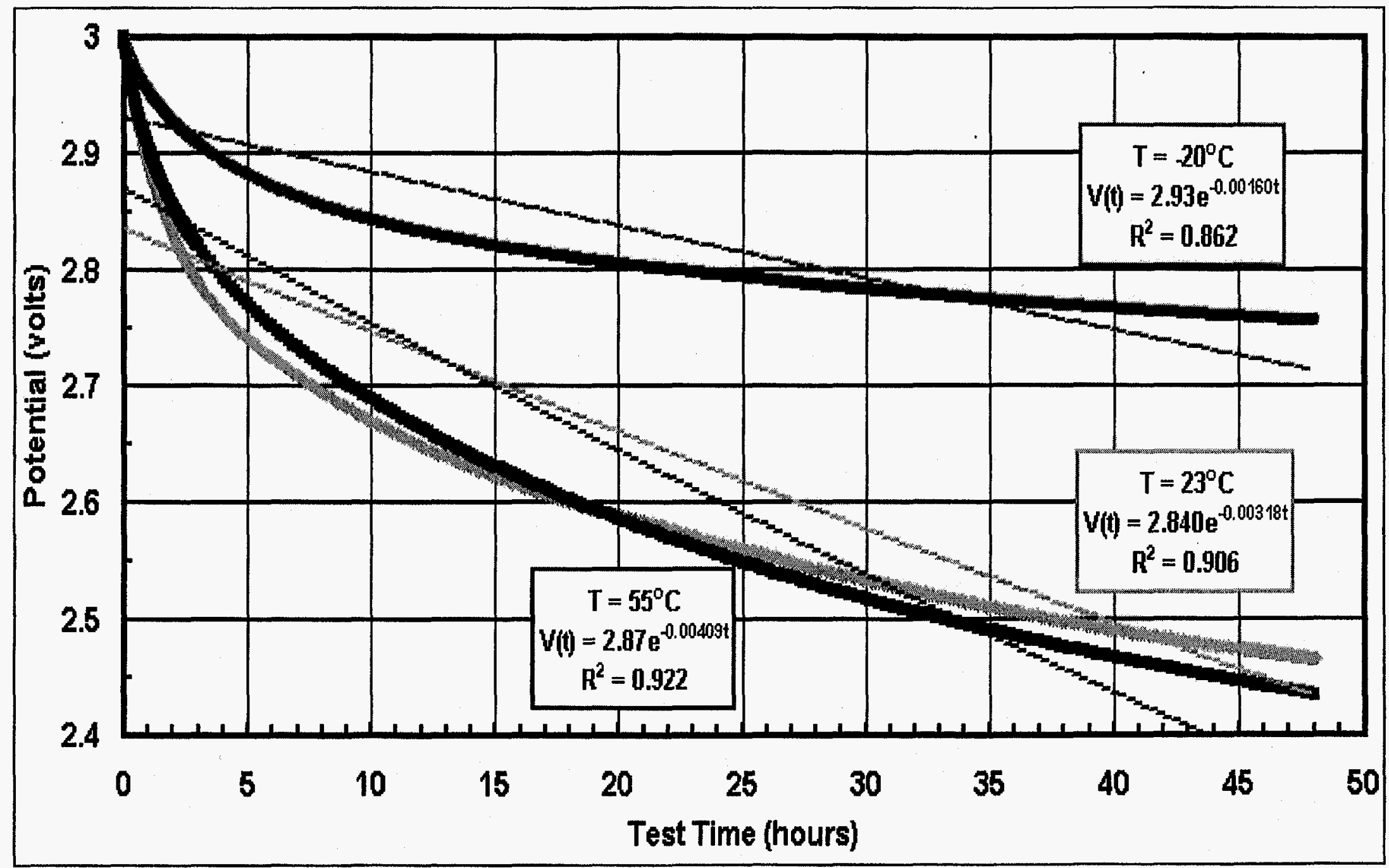

Figure A-115. Self-discharge test data for capacitor \#1022 as a function of temperature. Exponential time fits to the voltage data are shown. 


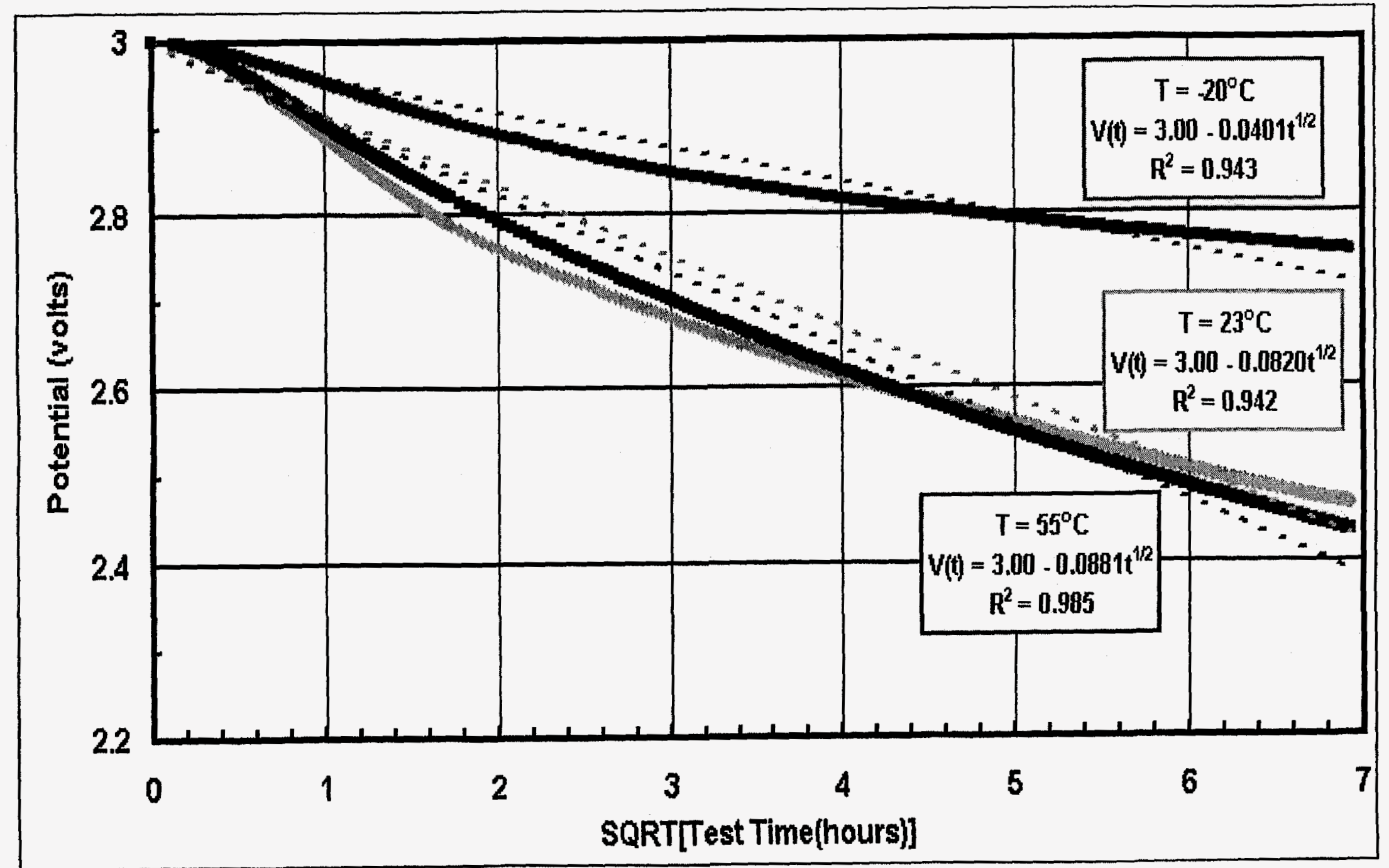

Figure A-116. Data from self-discharge test for capacitor \#1022 as a function of temperature. Fits to voltage data in the form of a square-root of the test time are shown. 


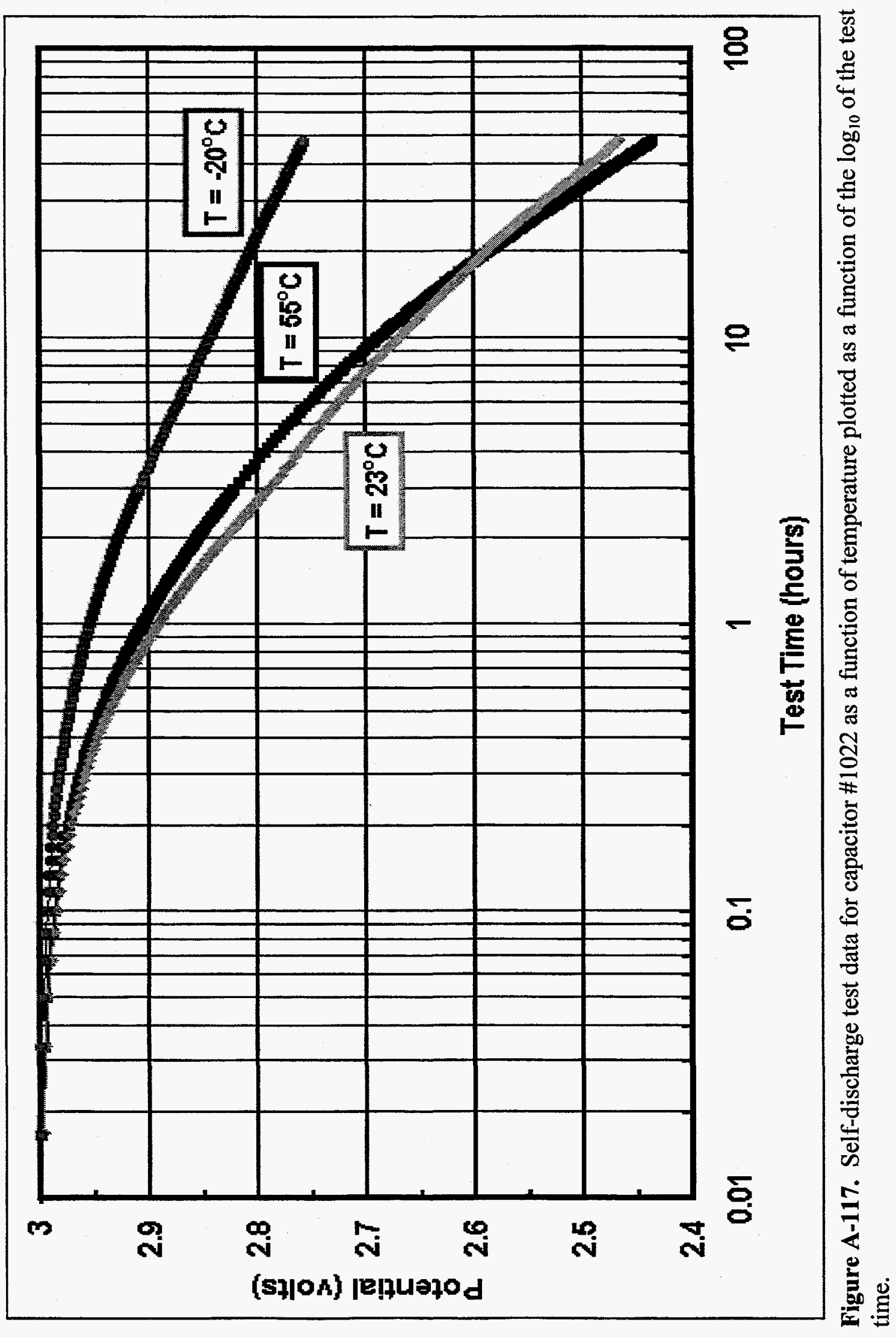




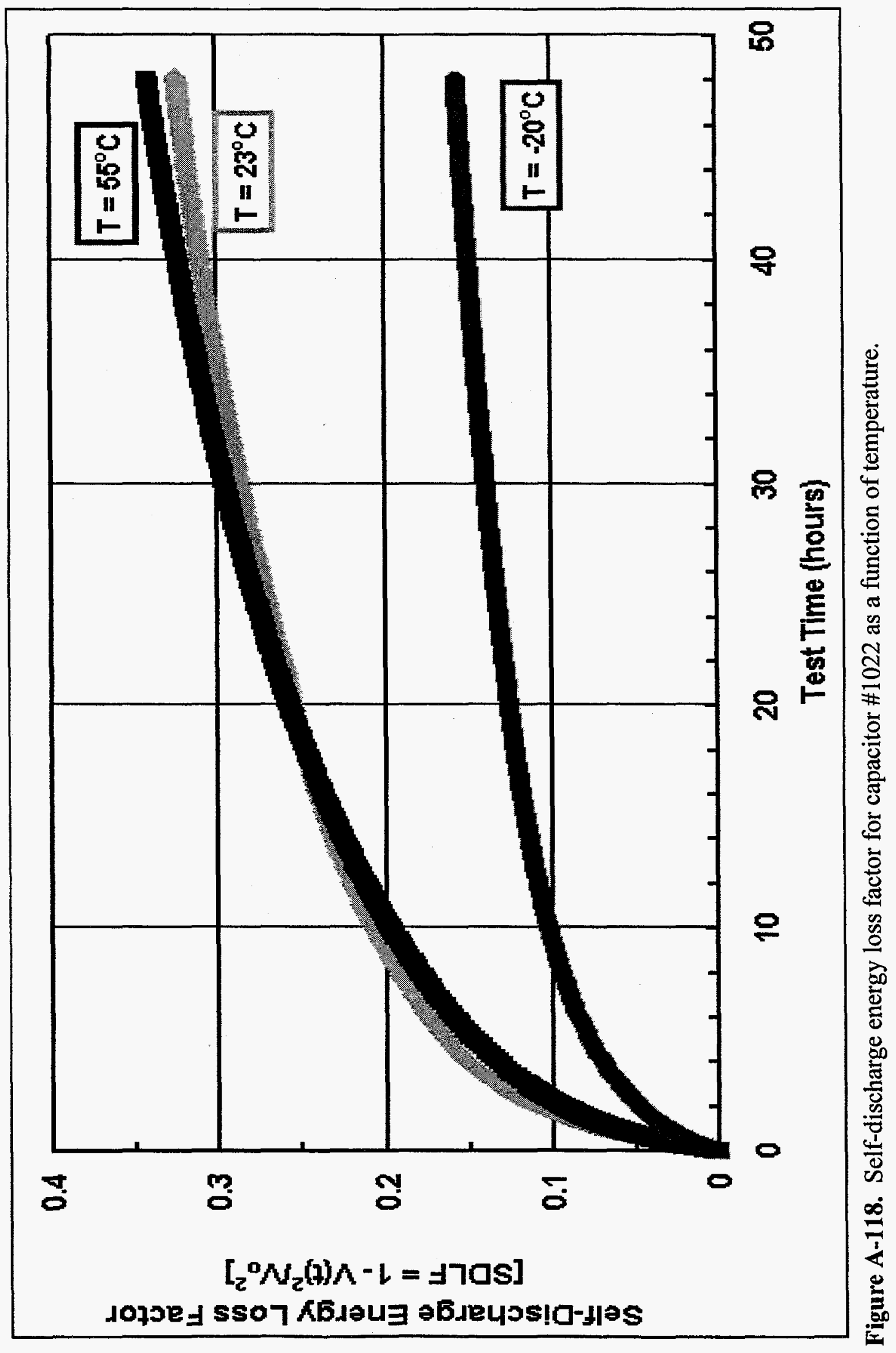




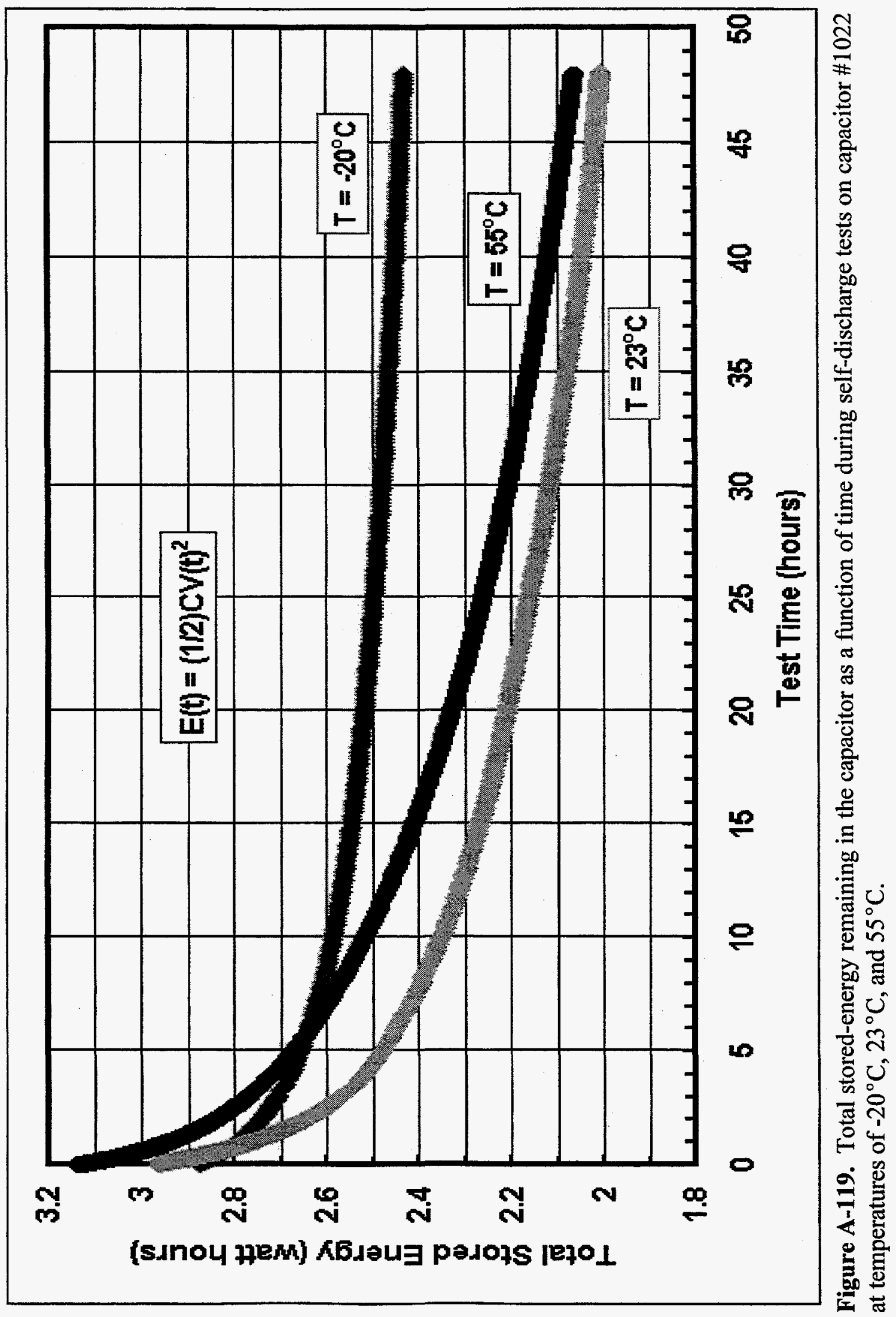




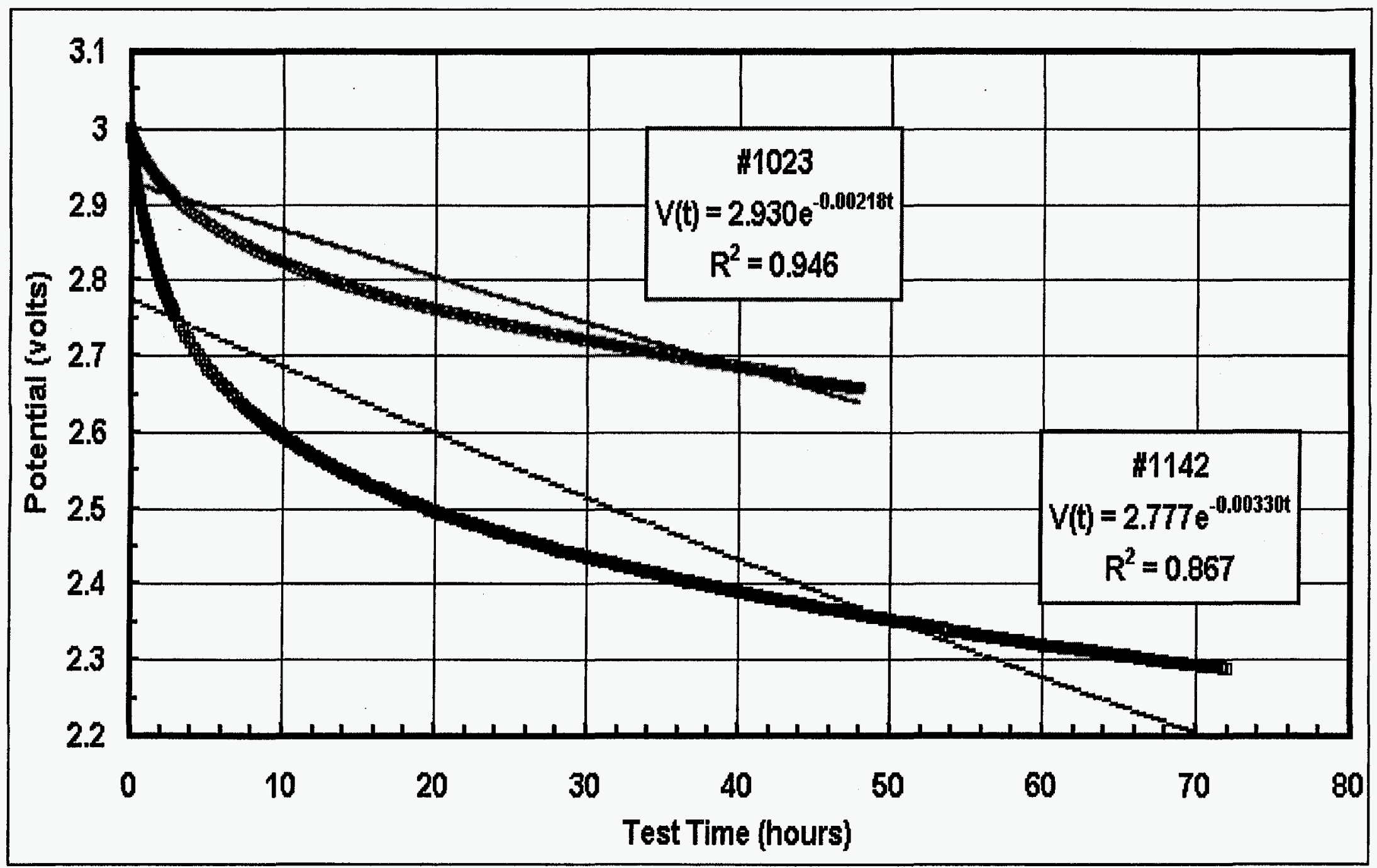

Figure A-120. Self-discharge test data for capacitors $\# 1142$ and $\# 1023$ at $23^{\circ} \mathrm{C}$. 


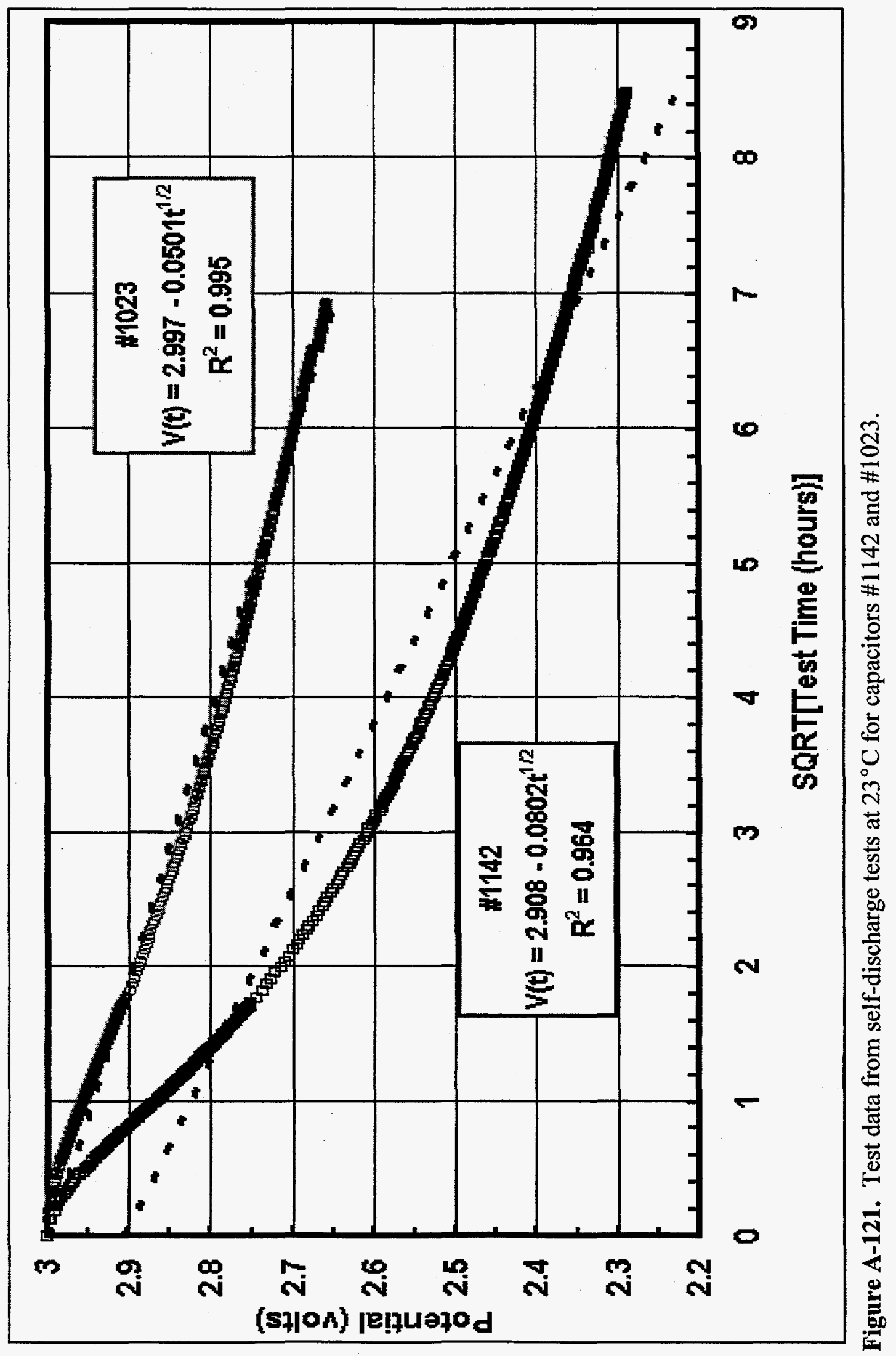




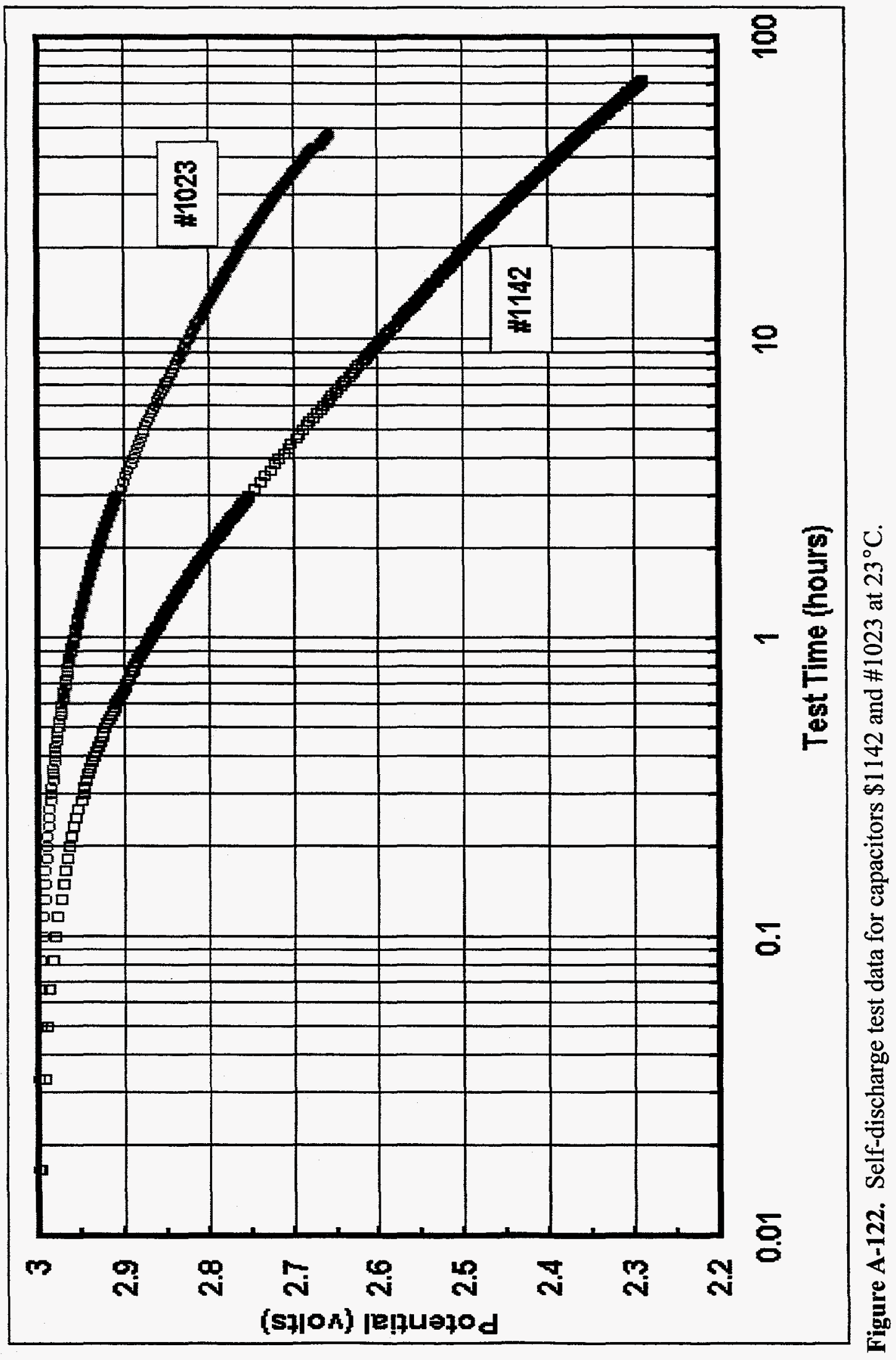




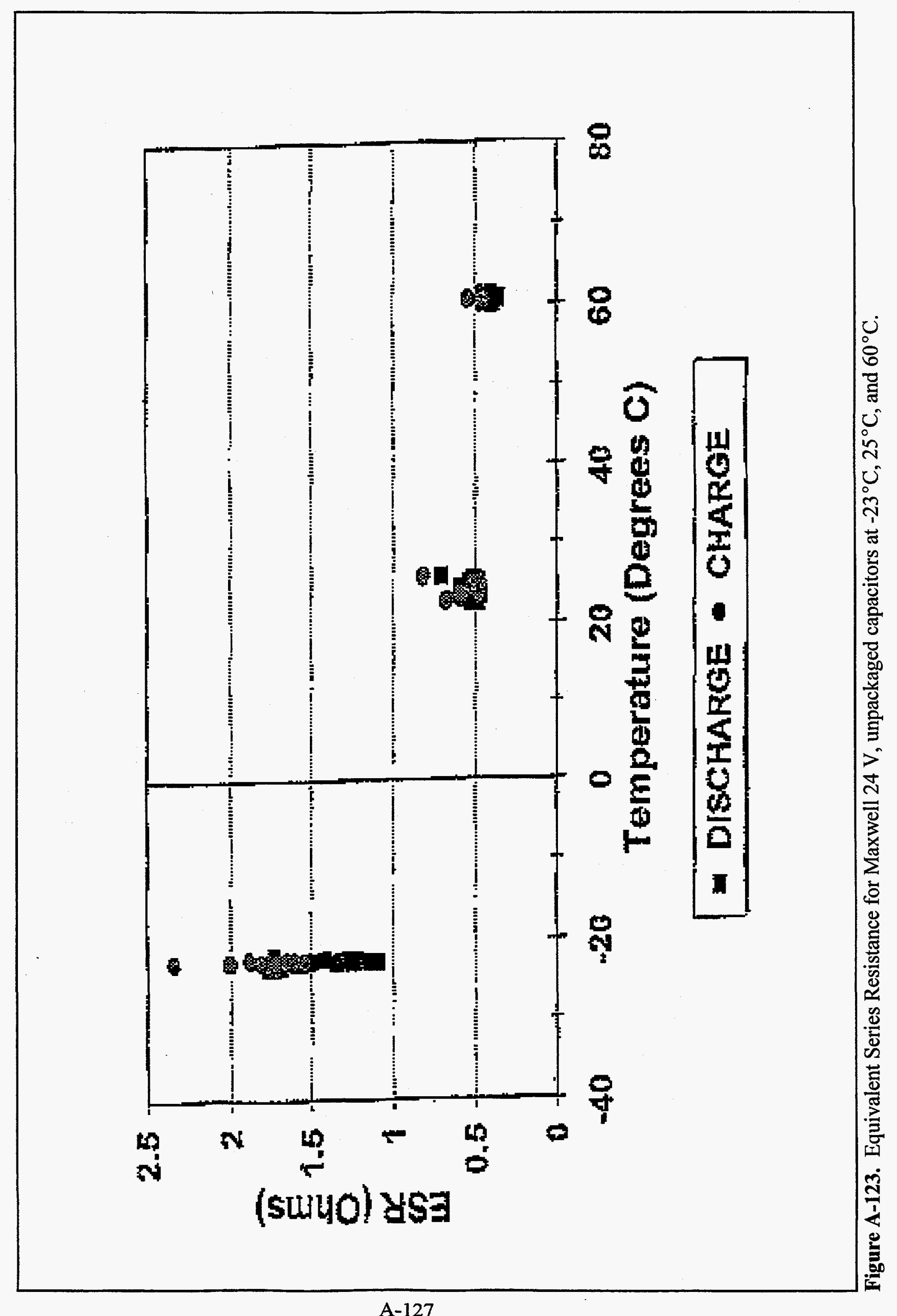




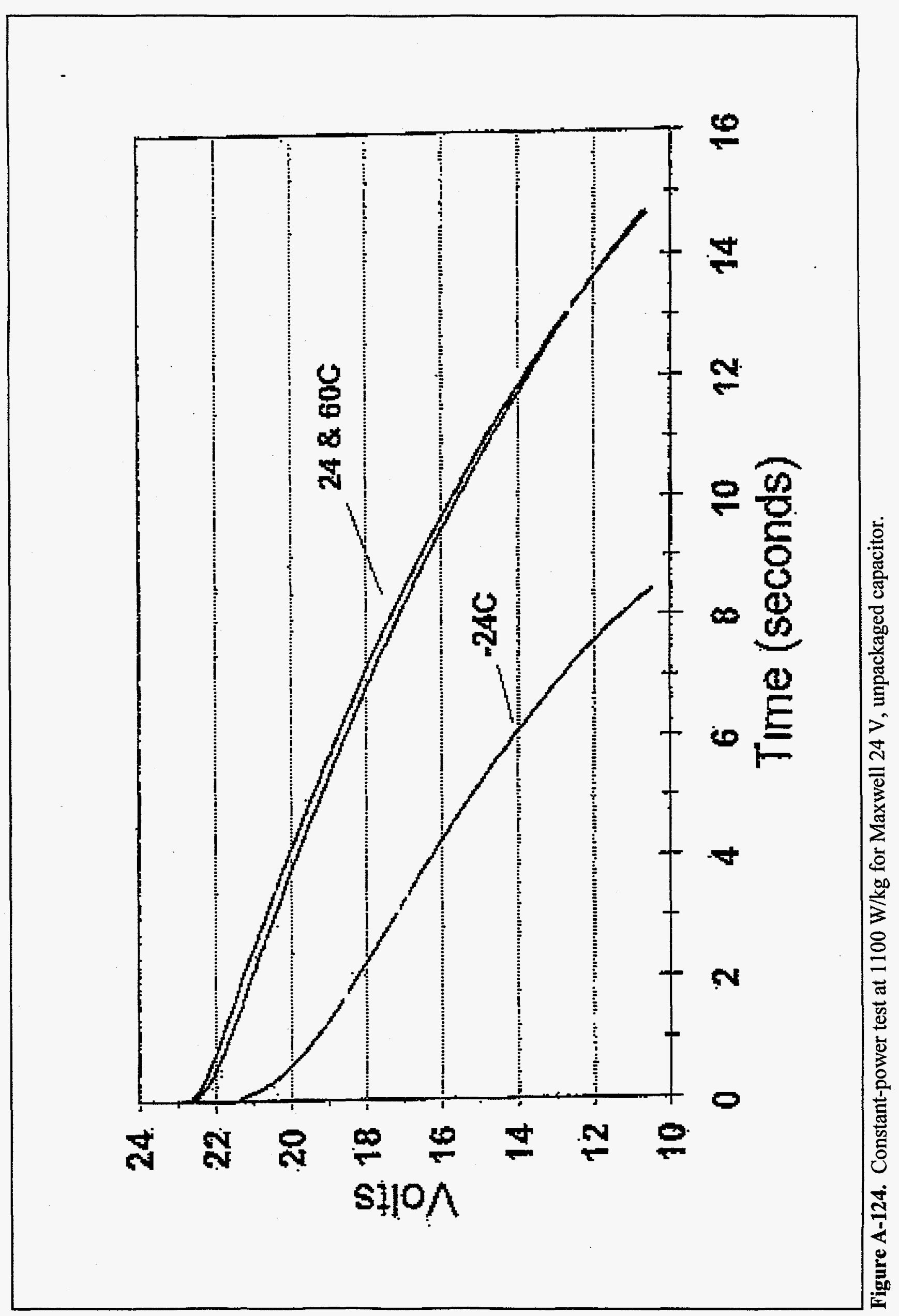




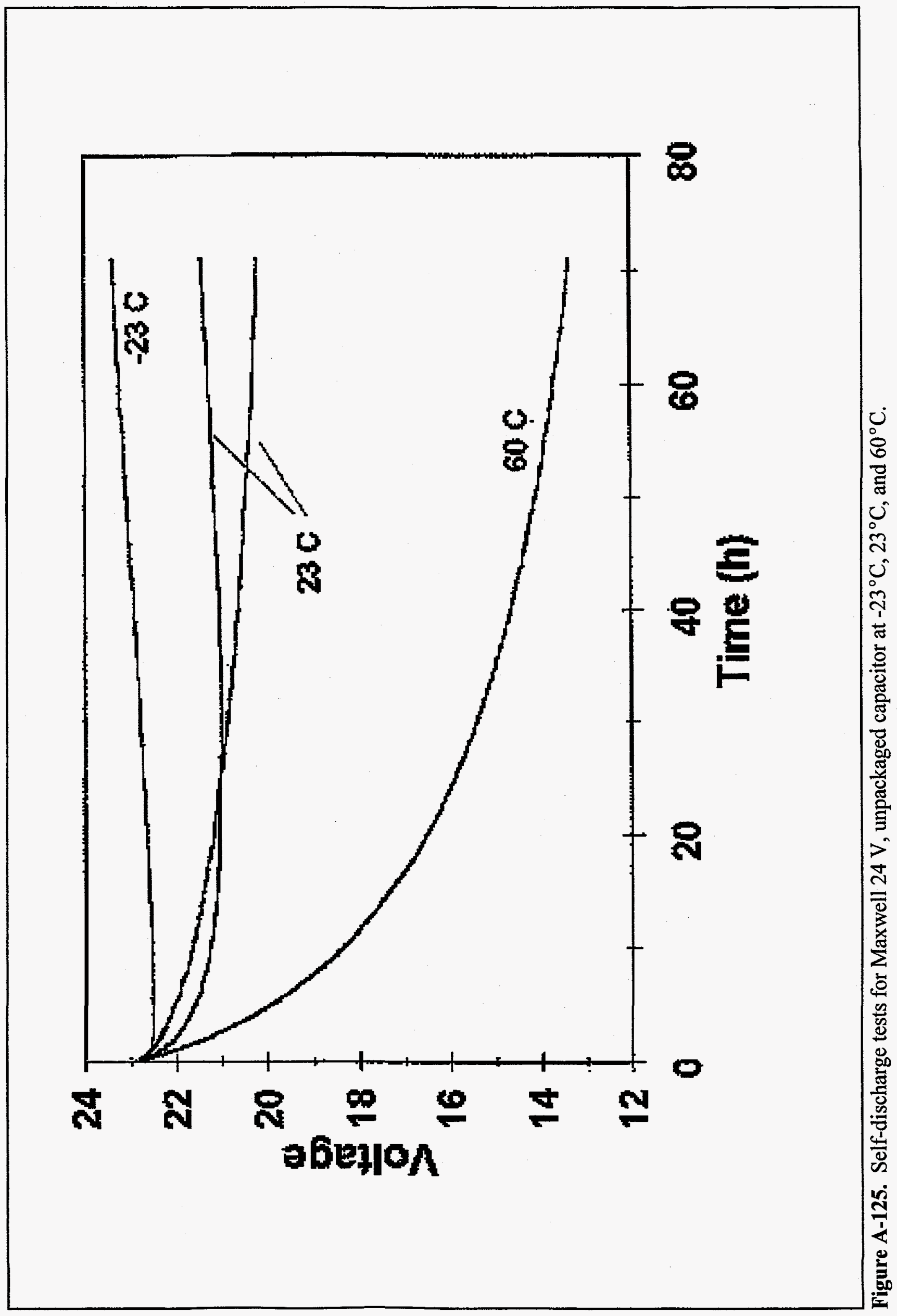




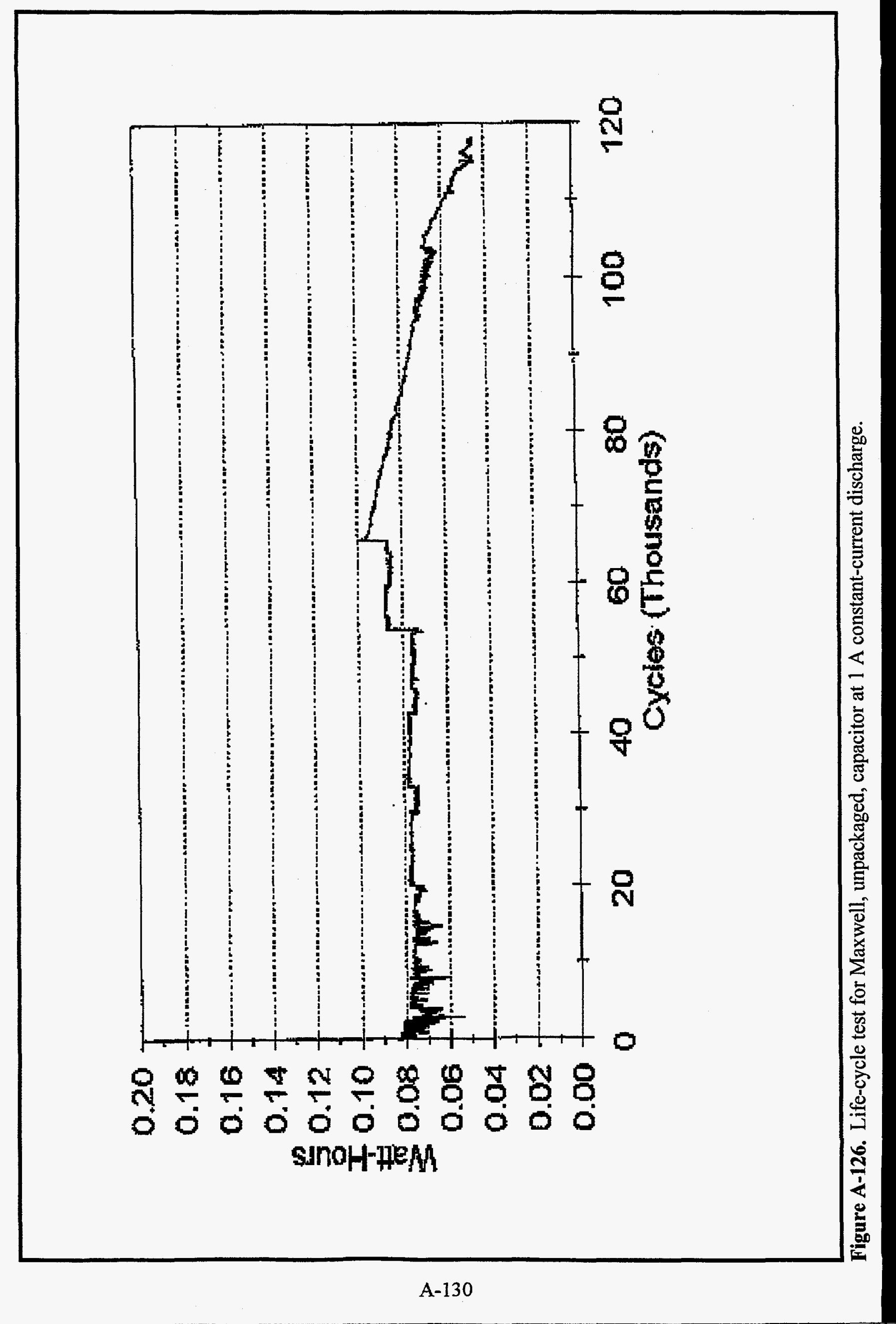




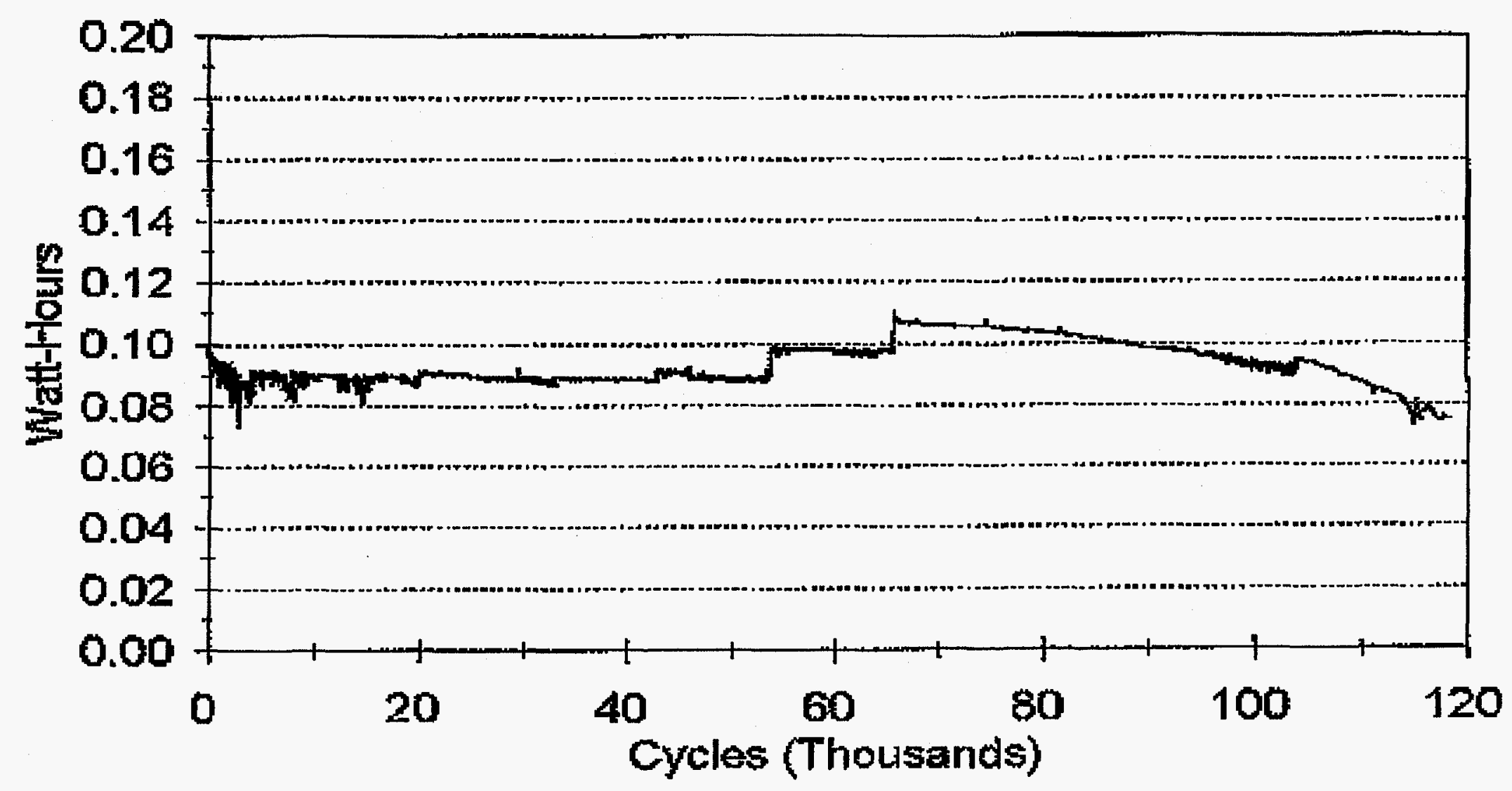

Figure A-127. Life-cycle test of Maxwell, unpackaged, $24 \mathrm{~V}$ capacitor at $1 \mathrm{~A}$ constant-current charge. 
A-132 
APPENDIX B

CAPACITOR THEORY AND CAPACITOR TESTING FUNDAMENTALS

B-1 
B-2 


\section{CAPACITOR TESTING FUNDAMENTALS}

\section{Definition}

\section{Capacitance is a measure of the ability of a device, a capacitor, to store energy in the form of separated charge or as an electric field.}

The capacitance, $\mathrm{C}$, of a capacitor is defined as the ratio of the charge $\mathrm{Q}$ (in units of coulombs, $1 \mathrm{e}^{-}=1.602 \times 10^{-19}$ coulomb) on either of two spatially separated conductors to the potential difference $\mathrm{V}_{\mathrm{ab}}$ (in volts) between the conductors as given by the general relation:

$$
\mathbf{C}=\mathbf{Q} / \mathbf{V}_{\mathbf{a b}}
$$

The unit of capacitance is the farad defined as one coulomb per volt. A farad is also defined to be one ampere-second/volt. A first-order model for an electrochemical capacitor includes a capacitor having a capacitance $C$ in series with a resistor with a resistance $R_{s}$ and a series inductor having an inductance $L$. A resistor $R_{p}$ in parallel with the capacitor can also be included in this model. $R_{s}$ is usually referred to as the equivalent series resistance (ESR) and contributes to energy loss during capacitor charging and discharging. $R_{p}$ simulates energy loss due to capacitor self-discharge and is often called the leakage-current resistance. $R_{p}$ is generally much larger than $R_{s}$ and can usually be neglected during the constant-current and constant-power tests. $\mathrm{L}$ is the inductance of the capacitor that results primarily from the construction of the capacitor and is usually small.

Capacitance (in farads) as defined above can be expressed as:

$$
\mathbf{C}=\mathbf{Q} / \mathbf{V}
$$

where $\mathrm{Q}$ is the charge on the capacitor in coulombs and $\mathrm{V}$ is the potential difference in volts. For a series $R C$ circuit (neglecting $R_{p}$ and $L$ ) and for an ideal capacitor that has a constant capacitance $C$ that does not depend on the capacitor voltage or charge the voltage measured across the RC-circuit as a function of time can be expressed as:

$$
\left[V_{0} \pm I_{s}\right]-V(t)=\left[Q_{0}-Q(t)\right] / C
$$

where $V_{0}$ is the initial voltage across the capacitor, $Q_{0}$ is the initial charge on the capacitor having a capacitance $\mathrm{C}$. The capacitance can also be labeled with the subscripts "c" and " $\mathrm{d}$ " to distinguish the case where the charging capacitance, $\mathrm{C}_{c}$, may be different from the discharging capacitance, $C_{d} \cdot Q(t)$ is the charge on the capacitor at time $t, I$ is the current flowing in the circuit and $R_{s}$ is the series resistance. (Note that the series resistance for the charge and discharge cycles are not necessarily the same, nor are they constants independent of the charge/discharge current and the temperature as has been observed in experimental studies.) The \pm sign in front of $I_{s}$ corresponds to a voltage jump that occurs at the initiation of charging of the capacitor, a " + " sign, and a voltage drop, a "-" sign, that occurs at the initiation of the discharging of the capacitor. 
During a constant-current charging test, $I=I_{c}=$ constant is set by the test during charging; $V_{0}=0$ if the capacitor is totally discharged or $V_{0}=V_{0}$ if the capacitor has been charged to a voltage $V_{o}$. $R_{s}$ will be replaced by $R_{c}$ to distinguish the charging ESR that may be different from that measured during discharging. For $Q_{o}=0$ then $Q(t)=I_{c} t$, or if $Q o=Q o$ then $Q(t)=Q_{0}$ $+I_{c} t$ so that the voltage as a function time during charging, $V_{c}(t)$ is:

$$
V_{c}(t)=\left(V_{o}+I_{c} R_{c}\right)+\left(I_{c} / C_{c}\right) t
$$

From equation (3) it can be seen that a plot of the capacitor voltage, $V_{c}(t)$, versus the test time (t) should be a straight line with an intercept at $t=0$ equal to $\left(V_{o}+I_{c} R_{c}\right)$ that will allow the determination of the equivalent series resistance for charging, $R_{c}$, i.e., $R_{c}=\left(\right.$ Intercept $\left.t_{c}-V_{o}\right) / I_{c}$. The slope of the straight line should be positive and equal to $\left(I_{c} / C_{c}\right)$ which will allow the determination of the capacitor's charging capacitance: $\mathrm{C}_{c}=\mathrm{I}_{\mathrm{c}} /$ Slope $_{\mathrm{c}}$. In practice, "real-world" capacitors are observed to deviate from the ideal-capacitor model in that the capacitor voltage as a function of charging time during a constant-current charge is nonlinear which can be attributed to a voltage (i.e., charge) dependent capacitance. Depending on the voltage dependence of the capacitance, this can cause positive and/or negative deviations from the linear relationship expressed in equation (3).

During a constant-current discharge test $V(t=0)=V_{0}$, and $R_{s}$ is redefined to be $R_{d}$ for discharging. $I_{d}=$ constant is set by the test and $I_{d} t$ corresponds to the amount of charge removed from the capacitor over a time period, $t$, so that $Q(t)=Q_{0}-I_{d} t$ resulting in the expression:

$$
V_{d}(t)=\left(V_{0}-I_{d} R_{d}\right)-\left(I_{d} / C_{d}\right) t
$$

As was the case for the constant-current charge test, a plot of the capacitor voltage, $V_{d}(t)$, as a function of the constant-current discharge test time, $t$, should result in a straight line with an intercept at $t=0$ equal to $\left(V_{0}-I_{d} R_{d}\right)$ from which the equivalent series resistance for discharging, $R_{d}$, can be determined by $R_{d}=\left(V_{o}-\right.$ Intercept $\left._{d}\right) / I_{d}$. The slope of the straight line should be negative and equal to $-\left(\mathrm{I}_{d} / \mathrm{C}_{\mathrm{d}}\right)$ and permits the determination of the capacitor's discharging capacitance: $C_{d}=-I_{d} /$ Slope $_{d}$.

The relationships expressed by equations (3) and (4) permit the determination of whether or not the capacitance is constant or if it depends on the voltage (charge) on the capacitor. Deviations of the charge or discharge voltages as a function of the charge or discharge test time from a straight line relationship would be indicative of a non-constant charge or discharge capacitance. Similarly, equation (1) would also enable this determination. Since $Q=C V$, then for a plot of $Q$, the charge on the capacitor as a function of voltage, versus the voltage on the capacitor, the resulting experimental data should lie on a straight line with a positive slope equal to $\mathrm{C}$.

The simple RC-series circuit used to model the capacitor during a constant-current charge cycle shows that the experimentally measured voltage, $\mathrm{V}_{\mathrm{c}}$, across the $\mathrm{RC}$ circuit is:

$$
\mathbf{V}_{\mathbf{c}}=\mathbf{V}_{\text {cap }, \mathrm{c}}+\mathbf{I}_{\mathrm{c}} \mathbf{R}_{\mathrm{c}}
$$


where $\mathrm{V}_{\text {cap, } \mathrm{c}}$ is the actual voltage across the capacitor during charging. Therefore, the voltage on the capacitor during charging is:

$$
\mathbf{V}_{\text {cap, } \mathrm{c}}=\mathbf{V}_{\mathrm{c}}-\mathbf{I}_{\mathrm{c}} \mathbf{R}_{\mathrm{c}}
$$

I.e., due to the voltage jump at the start of the charge cycle, the actual voltage across the capacitor is equal to the experimentally measured voltage minus the $I_{c} R_{c}$ voltage jump. The charge on the capacitor during charging, $Q_{c}$, is equal to $Q_{c}=Q_{o}+Q\left(V_{c}\right.$ or $\left.t\right)$ where $Q_{0}$ is the initial charge on the capacitor and $Q\left(V_{c}\right.$ or $\left.t\right)$ is the charge added to the capacitor as a function of time (or as a function of the measured voltage, $\mathrm{V}_{\mathrm{c}}$ ) during the charging cycle. Thus, the capacitance as a function of the voltage on the capacitor is:

$$
C_{c}\left(V_{c} \text { or } t\right)=\left[Q_{0}+Q\left(V_{c} \text { or } t\right)\right] /\left[V_{c}-I_{c} R_{c}\right]
$$

It should be noted that if the charging cycle is not started when $Q_{0}=O$, i.e., the voltage and charge on the capacitor are not zero, then the total charge on the capacitor, $\left[Q_{o}+Q\left(V_{c}\right.\right.$ or $\left.\left.t\right)\right]$, may not be known and the capacitance as a function of the measured voltage cannot accurately be determined. Experimentally, the most accurate determination of the capacitance of the capacitor would be for those measurements where the charge cycle starts at zero volts (i.e., no charge on the capacitor) and ends at the specified maximum voltage of the capacitor. Similarly, the discharge cycle should begin at the specified maximum voltage of the capacitor and end at zero volts.

For the simple $\mathrm{RC}$-series circuit, the experimentally measured voltage, $\mathrm{V}_{\mathrm{d}}$, across the $\mathrm{RC}$ circuit during a constant-current discharge cycle is:

$$
\mathbf{V}_{\mathbf{d}}=\mathbf{V}_{\text {cap,d }}-\mathbf{I}_{\mathrm{d}} \mathbf{R}_{\mathrm{d}}
$$

Where $\mathrm{V}_{\text {cap,d }}$ is the actual voltage across the capacitor during discharge. Therefore, the voltage on the capacitor during discharging is:

$$
\mathbf{V}_{\text {cap,d }}=\mathbf{V}_{\mathrm{d}}+\mathbf{I}_{\mathrm{d}} \mathbf{R}_{\mathrm{d}}
$$

I.e., due to the voltage drop at the start of the discharge cycle, the actual voltage across the capacitor is equal to the experimentally measured voltage plus the $I_{d} R_{d}$ voltage drop. The charge on the capacitor during discharging, $Q_{d}$, is equal to $Q_{d}=Q_{0}-Q\left(V_{d}\right.$ or $\left.t\right)$ where $Q_{0}$ is the initial charge on the capacitor and $\mathrm{Q}\left(\mathrm{V}_{\mathrm{d}}\right.$ or $\left.\mathrm{t}\right)$ is the charge removed from the capacitor as a function of time (or as a function of the measured voltage, $V_{d}$ ) during the discharge cycle. Thus, the capacitance as a function of the voltage on the capacitor is:

$$
C_{d}\left(V_{d} \text { or } t\right)=\left[Q_{o}-Q\left(V_{d} \text { or } t\right)\right] /\left[V_{d}+I_{d} R_{d}\right]
$$


It should be noted that the initial charge on the capacitor is that amount of charge added during the charging cycle. In order accurately to determine $Q_{0}$, the charge on the capacitor prior to the start of the discharge, the experimental charging voltage range should be the same as the discharge voltage range.

As mentioned above, the terms $\left(V_{o}+I_{c} R_{c}\right)$ for constant-current charging and $\left(V_{0}-I_{d} R_{d}\right)$ for constant-current discharging can be used to calculate the Equivalent Series Resistance (ESR) during charging and discharging of the capacitor. This is known as the $\mathrm{IR}_{\text {step }}$ method that monitors the voltage on the capacitor immediately after the current to/from the capacitor has been changed. In practice, the first measurement data point is several to several tens of milliseconds after the start of the constant-current charge or discharge step, which is usually accurate enough for the determination of the ESR value as will be described below in the section on Capacitor Test Procedures (DC Tests).

\section{Energy and Power Supplied by a Capacitor}

For a capacitor having a capacitance $C$ and charged to an initial voltage $V_{0}$ that is then discharged to a final voltage $V_{f}$, the energy $E_{d}$ (in joules) extracted is:

$$
E_{d}=(1 / 2) C V_{o}^{2}\left[1-\left(V_{f} / V_{o}\right)^{2}\right]
$$

This equation assumes that the ESR of the capacitor is zero. For example, if the capacitor is discharged to $1 / 2$ of its initial voltage, $V_{f}=1 / 2 V_{o}$, then the energy withdrawn is $3 / 4(75 \%)$ of the maximum available, i.e.,:

$$
E_{d}=(3 / 4)(1 / 2) C V_{0}^{2}
$$

Similarly, the total energy initially stored in the capacitor is, when $V_{f}=0$ :

$$
E_{\mathrm{T}}=(1 / 2) C V_{0}^{2}
$$

Equations (11) to (13) have not taken into account that the capacitance may not be a constant but may depend on the voltage (charge) on the capacitor, and also that the voltage (charge) dependence of the capacitance during charging may be different from that during discharging.

If the influence of the equivalent series resistance is taken into account (assuming a series-RC circuit) then upon initiation of a constant-current discharge the actual voltage of the capacitor is reduced by the IR-drop due to the series resistance:

$$
\mathbf{V}_{\mathrm{o}, \mathrm{d}}=\mathbf{V}_{\mathrm{o}}-\mathbf{I}_{\mathrm{d}} \mathbf{R}_{\mathrm{d}}
$$

where $V_{o}$ is the initial voltage of the capacitor immediately before the initiation of the constantcurrent discharge of magnitude $I_{d}$ and the capacitor has a constant-current discharge ESR equal to $R_{d}$. The discharge energy expression, equation (11), then becomes:

$$
E_{d}=(1 / 2) C_{d}\left(V_{o}-I_{d} R_{d}\right)^{2}\left\{1-\left[V_{f}\left(V_{o}-I_{d} R_{d}\right)\right]^{2}\right\}
$$


with $V_{f} \leq\left(V_{o}-I_{d} R_{d}\right)$. When $V_{f}=0$ then the total energy that can be extracted from the capacitor, $E_{\mathrm{T}}$, is, from equation (13), equal to:

$$
\mathbf{E}_{\text {T,extractable }}=(1 / 2) C_{d}\left(V_{0}-I_{d} R_{d}\right)^{2}
$$

This relation shows the importance of having as low as possible discharge ESR, $R_{d}$, in order to extract the maximum amount of energy that was initially stored in the capacitor. The capacitance has been defined to be the discharge capacitance, $C_{d}$, to show that the discharge capacitance may be different from the charge capacitance, $\mathrm{C}_{\mathrm{c}}$.

Power, $\mathrm{P}$ [in watts, where 1 watt $=(1$ ampere $)(1$ volt $)=(1$ coulomb $/$ second $)(1$ joule/coulomb) $=1$ joule/second], is defined to be equal to the energy, in joules $=3600 \mathrm{~W} \cdot \mathrm{h}$, that is expended over a time interval $\Delta \mathrm{t}$ (in seconds):

$$
\mathbf{P}=\mathbf{E} / \Delta \mathbf{t}
$$

Discharge power, $P_{d}$, can also be expressed as:

$$
\mathbf{P}_{\mathrm{d}}=\mathbf{I}_{\mathrm{d}} \mathbf{V}_{\mathrm{o}, \mathrm{d}}
$$

where $I_{d}$ is the constant-current discharge current and $V_{o, d}$ is the initial voltage on the capacitor prior to initiation of the discharge. Equation (14) is also relevant here as the initial voltage of the capacitor is reduced by the $I_{d} R_{d}$ loss so that:

$$
\mathbf{P}_{\mathbf{d}}=\mathbf{I}_{\mathbf{d}} \mathbf{V}_{\mathrm{o}}-\mathbf{I}_{\mathrm{d}}^{2} \mathbf{R}_{\mathrm{d}}
$$

which again illustrates that a low discharge ESR of the capacitor is very desirable.

Miller [10,11] and Conway [11,12] have discussed several issues pertaining to the energy and power capabilities of capacitors. We will reproduce and expand on their discussions in the following treatment. Using equation (19), the maximum power attainable during a constantcurrent discharge can be derived by differentiating this equation with respect to $I_{d}$, setting the expression equal to zero, and then solving for $I_{d}$ in terms of $V_{o}$ and $R_{d}$ :

$$
\begin{gathered}
d P_{d} / d I_{d}=0=V_{0}-2 I_{d} R_{d} \\
I_{d, \max }=V_{0} /\left(2 R_{d}\right)
\end{gathered}
$$

Upon substitution of this current into equation (19) the maximum discharge power, $P_{d, \max }$, can be found:

$$
\mathbf{P}_{\mathrm{d}, \max }=\mathbf{V}_{\mathrm{o}}^{2} /\left(4 \mathbf{R}_{\mathrm{d}}\right)
$$

Since from equation (13) the total energy initially stored in the capacitor is equal to $(1 / 2) \mathrm{C}_{\mathrm{d}} \mathrm{V}_{\mathrm{o}}^{2}$, it then follows that $V_{o}^{2}=2 E_{T} / C_{d} . P_{d, m a x}$ in terms of $E_{T}, C_{d}$ and $R_{d}$ can then be given by: 


$$
\mathbf{P}_{\mathrm{d}, \max }=\mathbf{E}_{\mathrm{T}} /\left(2 \mathrm{R}_{\mathrm{d}} \mathrm{C}_{\mathrm{d}}\right)
$$

The maximum discharge energy that can be extracted from the capacitor that corresponds to this maximum discharge power is found by substituting $I_{d, \max }$ [equation (21)] for $I_{d}$ in equation (16):

$$
\mathrm{E}_{\mathrm{d}, \mathrm{at} \max }=\mathrm{C}_{\mathrm{d}} \mathrm{V}_{\mathrm{o}}{ }^{2} / 8=(1 / 4) \mathrm{E}_{\mathrm{T}}
$$

Using equation (11), the energy given by equation (24) for the discharge energy corresponding to the maximum discharge power would be that energy produced by discharging the capacitor from $\mathrm{V}_{0}$ to $(3 / 4)^{1 / 2} \mathrm{~V}_{0}$, i.e., from $\mathrm{V}_{0}$ to $\sim 0.866 \mathrm{~V}_{0}$.

Expressions (22) and (23) also illustrate the point that in order to have the capacitor provide the maximum amount of power then the discharge ESR, and the (ESR)(capacitance) product, i.e., the $\mathrm{R}_{d} \mathrm{C}_{\mathrm{d}}$ time-constant of the capacitor (discussed further below), should be as low as possible. The situation just described, according to Miller [5], corresponds to a situation where $P_{d, \max }$ is the maximum power that can be delivered to a load resistor, $R_{L}$, whose resistance is equal to the discharge ESR of the capacitor, $R_{d}$, a so-called matched load. With this load, the capacitor's voltage immediately drops from $V_{0}$ to $V_{0} / 2$ when the discharge begins. That this is the case can be seen from the usual relation $P=I V=V^{2} / R$ where if $V=V_{0} / 2$ then $P=V_{0}{ }^{2} /(4 R)$ which is relation (22). This result can also be derived by using equation (19) and substituting for $I_{d}$ the current flowing through the series connected load, $R_{L}$, and the discharge ESR of the capacitor, $R_{d}$, i.e., $I_{d}=V_{o} /\left(R_{L}+R_{d}\right)$ :

$$
\begin{gathered}
\mathbf{P}=\mathbf{V}_{0}{ }^{2} /\left(\mathbf{R}_{\mathrm{L}}+\mathbf{R}_{\mathrm{d}}\right)-\mathbf{V}_{\mathrm{o}}{ }^{2} \mathbf{R}_{\mathrm{d}} /\left(\mathbf{R}_{\mathrm{L}}+\mathbf{R}_{\mathrm{d}}\right)^{2} \\
\mathbf{d P} / \mathbf{d R}_{\mathrm{L}}=\mathbf{0}=\mathbf{V}_{\mathrm{o}}{ }^{2}\left[-\left(\mathbf{R}_{\mathrm{L}}+\mathbf{R}_{\mathrm{d}}\right)^{-2}+2 \mathbf{R}_{\mathrm{d}}\left(\mathbf{R}_{\mathrm{L}}+\mathbf{R}_{\mathrm{d}}\right)^{-3}\right] \\
\mathbf{R}_{\mathrm{L}}=\mathbf{R}_{\mathrm{d}}
\end{gathered}
$$

which yields equation (22) when this result is substituted into equation (25).

The concept of an RC time-constant as discussed above also enters into situations where a simple series-RC circuit is short-circuited. In this case a capacitor initially charged to a total charge of $Q_{0}$ and having an initial voltage $V_{o}$ with a capacitance $C$ is connected across a resistor having a resistance $R_{s}$ (for example, the equivalent series discharge resistance, $R_{d}$, of the capacitor). This situation causes the capacitor to discharge as a function of time. The time dependence of the charge, voltage, and current flowing across the series connected resistor, $R_{s}$, are expressed by the relations:

$$
\begin{aligned}
\mathbf{Q} & =\mathbf{Q}_{0} \exp \left[-t /\left(R_{s} C\right)\right] \\
\mathbf{V} & =\mathbf{V}_{0} \exp \left[-t /\left(R_{s} C\right)\right] \\
I & =I_{0} \exp \left[-t /\left(R_{s} C\right)\right]
\end{aligned}
$$

where $t$ is the time in seconds for the resistance, $R_{\mathrm{s}}$ in ohms and the capacitance, $C$, in farads. 
The initial current is $I_{0}=Q_{0} /\left(R_{s} C\right)=V_{d} / R_{s}$. The time duration for $Q, V$ and $I$ to decrease to half their initial values.(i.e., to $\mathrm{Q}_{\delta} / 2, \mathrm{~V}_{\delta} / 2$ and $\left.\mathrm{I}_{\mathrm{o}} / 2\right)$ is $\mathrm{R}_{\mathrm{s}} \mathrm{C} \ln (2)=(0.6932) \mathrm{R}_{\mathrm{s}} \mathrm{C}$ seconds. Expression (29) also pertains to the situation where the capacitor can be modeled with a resistor, $R_{p}$, in parallel with the capacitor in addition to the series resistor $R_{s}\left(R_{p}\right.$ is generally much greater than $\left.R_{s}\right)$. This situation would be relevant for the self-discharge of the capacitor where even under open-circuit conditions the capacitor would discharge due to the parallel resistor. (The leakagecurrent test described below offers an experimental means of measuring the value of the parallel resistor for this model.)

The energy and power mass and volume densities corresponding to the above expressions can be obtained by dividing, respectively, by the mass (in $\mathrm{kg}$ ) or volume (in liters) of the capacitor. The energy mass density and power mass density are usually referred to as the specific energy and specific power respectively.

There is also interest in the efficiency with which a capacitor can be used to supply energy and power to a load. Miller [11] has given the efficiency of a capacitor, $n$, for various conditions such as constant-current charging at a current of $I_{c}$ where the capacitor has a charging ESR equal to $R_{c}$ :

$$
\mathbf{n}_{\text {constant-current charge }}=\left(\mathbf{V}_{\mathrm{o}}-\mathbf{I}_{\mathrm{c}} \mathbf{R}_{\mathrm{c}}\right) /\left(\mathbf{V}_{\mathrm{o}}+\mathbf{I}_{\mathrm{c}} \mathbf{R}_{\mathrm{c}}\right)
$$

The relation for a pure resistive load, $R_{L}$, during discharge for a capacitor modeled with a series ESR equal to $R_{d}$ is:

$$
\mathbf{n}_{\text {resistive discharge load }}=\mathbf{R}_{\mathbf{L}} /\left(\mathbf{R}_{\mathbf{L}}+\mathbf{R}_{\mathrm{d}}\right)
$$

and for a constant-current discharge, $I_{d}$, that includes a constant-current discharge ESR, $R_{d}$, of the capacitor:

$$
\mathbf{n}_{\text {constant-current discharge }}=\left(V_{0}-I_{d} R_{d}\right) /\left(V_{0}+I_{d} R_{d}\right)
$$

where $V_{o}$ is the final voltage to which the capacitor is charged, $I_{c}$ and $R_{c}$ are the charging current and the charging ESR of the capacitor respectively; $R_{L}$ is the pure resistive discharge load; and $I_{d}$ and $R_{d}$ are the constant-current discharge current and the capacitor's discharge ESR. It should be noted that $R_{c}$ and $R_{d}$ are not necessarily equal and are often a function of the constant-current charge and discharge current. These expressions, in conjunction with the above derived relations for the maximum power, allow the determination of the maximum power that can be supplied by the capacitor at a specified efficiency. Using equation (33) for the case of a constant-current discharge and solving for $I_{d}$ in terms of the efficiency, $n, V_{0}$ and $R_{d}$ results in the relation (after redefining $\mathrm{n}_{\text {constant-current discharge }} \equiv \mathrm{n}_{\text {ced }}$ ):

$$
\mathbf{I}_{\mathrm{d}}=\left[\left(\mathbf{1}-\mathbf{n}_{\mathrm{ccd})}\left(\mathbf{1}+\mathbf{n}_{\mathrm{ccd})}\right]\left(\mathbf{V}_{\mathbf{0}} / \mathbf{R}_{\mathrm{d}}\right)\right.\right.
$$

This expression for $I_{d}$ upon substitution into equation (16) for the energy and equation (19) for the power gives the energy and power available from a capacitor that has a constant discharge capacitance equal to $C_{d}$ during a constant-current discharge as a function of the efficiency, $n_{\text {cod- }}$ 
The energy available at an efficiency $n_{\text {ccd }}$ is:

$$
\mathbf{E}_{\text {at efficiency nced }}=\left[2 \mathbf{n}_{\text {ced }} /\left(1+\mathbf{n}_{\text {ced }}\right)\right]^{2}\left[\left(\frac{1}{2}\right) \mathbf{C}_{\mathrm{d}} \mathbf{V}_{0}^{2}\right]
$$

and,

$$
\mathbf{E}_{\text {at efficiency necd }}=\left[2 \mathbf{n}_{\text {ced }} /\left(1+\mathbf{n}_{\text {ccd }}\right)\right]^{2} \mathbf{E}_{T}
$$

from equation (13). From equation (19), the power available at an efficiency $n_{c c d}$ is,

$$
\mathbf{P}_{\text {at efficiency nced }}=\left[\left(2 \mathbf{n}_{\mathrm{ccd}}\right)\left(1-\mathbf{n}_{\mathrm{ccd}}\right) /\left(1+\mathbf{n}_{\mathrm{ccd}}\right)^{2}\right]\left(\mathbf{V}_{\mathrm{o}}{ }^{2} / \mathbf{R}_{\mathrm{d}}\right)
$$

and,

$$
P_{\text {at efficiency necd }}=\left[\left(2 n_{\text {ced }}\right)\left(1-n_{c c d}\right) /\left(1+n_{c c d}\right)^{2}\right]\left[2 E_{T} /\left(R_{d} C_{d}\right)\right]
$$

also from equation (13). The power at an efficiency $n_{\text {ccd }}$ can also be expressed in terms of the energy at an efficiency $\mathrm{n}_{\text {ccd }}$ by using equations (36) and (38) which results in:

$$
\mathbf{P}_{\text {at efficiency necd }}=\left[\left(\begin{array}{ll}
-\mathbf{n}_{\text {ced }} \\
)
\end{array} / \mathbf{n}_{\text {ced }}\right]\left[\mathbf{E}_{\text {at efficiency nced }} /\left(\mathbf{R}_{\mathrm{d}} \mathbf{C}_{\mathrm{d}}\right)\right]\right.
$$

The efficiency factors $\left[2 \mathrm{n}_{\mathrm{ccd}} /\left(1+\mathrm{n}_{\mathrm{ccd}}\right)\right]^{2},\left[\left(2 \mathrm{n}_{\mathrm{ccd}}\right)\left(1-\mathrm{n}_{\mathrm{ccd}}\right) /\left(1+\mathrm{n}_{\mathrm{ccd}}\right)^{2}\right]$ and $\left[\left(1-\mathrm{n}_{\mathrm{ccd}}\right) /\left(\mathrm{n}_{\mathrm{ccd}}\right)\right]$ are plotted in Figures B-1 and B-2 as a function of $n_{\text {ccd }}$ to more readily visualize their dependence on the efficiency. When the efficiency is 1 (i.e., 100\%) then the energy available is the total amount stored in the capacitor, $\mathrm{E}_{\mathrm{T}}$, but the available power is zero. This is because an efficiency of 1 $(100 \%)$ implies that the value of the series resistor is zero, i.e., there are no $I^{2} R-$ losses in the circuit due to the discharge ESR of the capacitor that means that this amount of power is lost and, thus, the total amount of power that could be provided by the capacitor cannot be supplied to the load with $100 \%$ efficiency. For $90 \%$ efficiency, the available energy is $E_{\text {available at } 90 \% \text { efficiency }}=$ $(0.8975)\left(1 / 2 \mathrm{C}_{\mathrm{d}} \mathrm{V}_{\mathrm{o}}{ }^{2}\right)=(0.8975) \mathrm{E}_{\mathrm{T}}$ and the available power is $\mathrm{P}_{\text {available at } 90 \% \text { efficiency }}=(0.04986)\left(\mathrm{V}_{\mathrm{o}}{ }^{2} / \mathrm{R}_{\mathrm{d}}\right)$ $=(0.09972)\left[E_{T} /\left(R_{d} C_{d}\right)\right]$. At $50 \%$ efficiency, $E_{\text {available at } 50 \% \text { efficiency }}=(0.4444)\left(1 / 2 C_{d} V_{o}^{2}\right)=(0.4444) E_{T}$ and $P_{\text {available at } 50 \% \text { efficiency }}=(0.2222)\left(V_{o}{ }^{2} / R_{d}\right)=(0.4444)\left[E_{T} /\left(R_{d} C_{d}\right)\right]$. From these relations it is again seen how important a low value of the RC-series time constant is for the maximum production of power by the capacitor.

Equations (37) and (38) can also be maximized with respect to $\mathrm{n}_{\mathrm{ccd}}$ to determine the maximum power available, i.e.:

$$
\begin{aligned}
& \mathrm{dP} / \mathrm{dn}_{\mathrm{ccd}}=\mathbf{0}=\left[2-\mathbf{6 n}_{\mathrm{ccd}}\right]\left\{\left(\mathrm{V}_{\mathrm{o}}^{2} / \mathbf{R}_{\mathrm{d}}\right) \text { or }\left[2 \mathrm{E}_{\mathrm{T}} /\left(\mathbf{R}_{\mathrm{d}} \mathrm{C}_{\mathrm{d}}\right)\right]\right\} \\
& \mathbf{n}_{\text {ced, } \max }=1 / 3
\end{aligned}
$$

The maximum power available, therefore, occurs when $n_{\text {cdd }}=1 / 3$ or $~ 33.3 \%$ efficiency and is equal to:

$$
P_{\text {at efficiency factor max }=33.3 \% \text { efficiency }}=(1 / 4)\left(V_{0}^{2} / R_{d}\right)
$$


and,

$$
P_{\text {at efficiency factor max }=33.3 \% \text { efficiency }}=(1 / 4)\left[2 E_{T} /\left(R_{d} C_{d}\right)\right]=E_{T} /\left(2 R_{d} C_{d}\right)
$$

Equations (42) and (43) correspond to equations (22) and (23) derived previously. From equations (37) and (38) with the use of equations (22) and (23) for $P_{\max }$ in terms of $V_{o}, R_{c}, C_{d}$ and $\mathrm{E}_{\mathrm{T}}$, the maximum power at a given efficiency can be derived:

$$
\begin{gathered}
\mathbf{P}_{\max \text { at efficiency nced }}=\left[\left(2 \mathbf{n}_{\mathrm{ccd}}\right)\left(1-\mathbf{n}_{\mathrm{ccd}}\right) /\left(1+\mathbf{n}_{\mathrm{ccd}}\right)^{2}\right](4 \mathrm{Pmax}) \\
\mathbf{P}_{\text {max at efficiency nced }}=\left[\left(2 \mathbf{n}_{\mathrm{ccd}}\right)\left(1-\mathbf{n}_{\mathrm{ccd}}\right) /\left(1+\mathbf{n}_{\mathrm{ccd}}\right)^{2}\right]\left(\mathbf{V}_{\mathrm{o}}^{2} / \mathbf{R}_{\mathrm{d}}\right) \\
\mathbf{P}_{\text {max at efficiency nced }}=\left[\left(2 \mathbf{n}_{\mathrm{ccd}}\right)\left(1-\mathbf{n}_{\mathrm{ccd}}\right) /\left(1+\mathbf{n}_{\mathrm{ccd}}\right)^{2}\right]\left[2 \mathrm{E}_{\mathrm{T}} /\left(\mathbf{R}_{\mathrm{d}} \mathrm{C}_{\mathrm{d}}\right)\right]
\end{gathered}
$$

The term $4\left[\left(2 n_{c c d}\right)\left(1-n_{c c d}\right) /\left(1+n_{c c d}\right)^{2}\right]$ in equation (44) which arises due to considerations of the efficiency (refer to Figures $1 \mathrm{~B}$ and $2 \mathrm{~B}$ ) reduces the maximum power that can be extracted from the capacitor at efficiencies $n_{\text {ccd }}<1 / 3$ and $n_{\text {ccd }}>1 / 3$. For example, at $90 \%$ efficiency the maximum power that can be extracted is 0.1995 or $\sim 1 / 5$ of $P_{\max }$, i.e., $P_{\max } \sim(1 / 5)\left[V_{d} /\left(4 R_{d}\right)\right]$ or, $\sim(1 / 5)\left[E_{T} /\left(2 R_{d} C_{d}\right)\right]$. From equation (33), where an expression for the maximum current was derived, it can also be determined that for a matched load [i.e., $R_{d} \equiv\left(R_{L}+R_{d}\right)=2 R_{d}$ for $R_{L}=R_{d}$ as defined for a matched load] the efficiency is only $33.3 \%$

Using the same approach, the maximum energy as a function of the efficiency can be derived using equations (35) and (36):

$$
\begin{gathered}
\mathbf{E}_{\text {max at efficiency necd }}=\left[2 \mathbf{n}_{\text {ced }} /\left(1+\mathbf{n}_{\text {ccd }}\right)\right]^{2}[4 \mathrm{Emax}] \\
\mathbf{E}_{\text {max at efficiency necd }}=\left[2 \mathbf{n}_{\text {ced }} /\left(1+\mathbf{n}_{\text {ced }}\right)\right]^{2} \mathbf{E}_{T}
\end{gathered}
$$

The efficiency factor $4\left[2 n_{\text {cod }} /\left(1+n_{c c d}\right)\right]^{2}$ in this case can increase the maximum energy that can be extracted from the capacitor as equation (24) was derived at an efficiency of $1 / 3$ or $-33.3 \%$ (refer to Figures 1B and 2B). This discussion of efficiency is important from the point of view of designing commercial systems that use capacitors as energy storage devices. The efficiency enters into discussions dealing with the energy and power supplied to the integrated system in that in order to use the capacitor in an optimum manner, considerations must be made as to how efficiently the capacitor is to be used. These considerations can have a major impact on the actual energy and power supplied by the capacitor to the integrated system.

An additional topic that is of interest regarding the energy and power capabilities of capacitors is the time dependence of the energy stored in the capacitor and the time dependence of the power produced by the capacitor under various combinations of resistive loads. For the case where the capacitor is modeled using only a series resistor, $R_{d}$, to represent the discharge ESR of the capacitor, then from equations (13), (18), (29) and (30) the energy stored in the capacitor, $E_{\text {cap }}(t)$, and the power produced by the capacitor, $P_{\text {cap }}(t)$, as a function of discharge time are given by: 


$$
\begin{gathered}
E_{c a p}(t)=(1 / 2) C_{d} V_{o}^{2} \exp \left[-2 t /\left(R_{d} C_{d}\right)\right] \\
E_{c a p}(t)=E_{T} \exp \left[-2 t /\left(R_{d} C\right)\right] \\
P_{c a p}(t)=\left(V_{o}^{2} / R_{d}\right) \exp \left[-2 t /\left(R_{d} C_{d}\right)\right] \\
P_{c a p}(t)=\left[2 E_{T} /\left(R_{d} C_{d}\right)\right] \exp \left[-2 t /\left(R_{d} C_{d}\right)\right]
\end{gathered}
$$

For expressions (49) to (52), if $R_{d}$ and $C_{d}$ are given in ohms and farads respectively, then time is in seconds. The time it takes $E_{\text {cap }}(t)$ and $P_{\text {cap }}(t)$ to decrease to $1 / 2$ of their initial values is given by $t_{1 / 2}=(1 / 2) R_{d} C_{d} \ln (2)=(0.3466) R_{d} C_{d}$ seconds. Graphs of the general function $\exp [-2 t /(R C)]$ for various values of $[2 /(\mathrm{RC})]$ as a function of time are presented in Figure $\mathrm{B}-3$ for reference. The case where a purely resistive load, $R_{L}$, is connected in series with the series resistance model of the capacitor leads to expressions for the time dependence of the power produced by the capacitor. By using the fact that the power dissipated in the load, $R_{L}$, and the ESR resistor, $R_{d}$ are:

$$
\begin{aligned}
& \mathbf{P}_{\mathrm{RL}}=\mathrm{I}^{2} \mathbf{R}_{\mathrm{L}}=\mathrm{V}^{2} \mathbf{R}_{\mathrm{L}} /\left(\mathbf{R}_{\mathrm{d}}+\mathbf{R}_{\mathrm{L}}\right)^{2} \\
& \mathbf{P}_{\mathbf{R d}}=\mathrm{I}^{2} \mathbf{R}_{\mathrm{d}}=\mathrm{V}^{2} \mathbf{R}_{\mathrm{d}} /\left(\mathbf{R}_{\mathrm{d}}+\mathbf{R}_{\mathrm{L}}\right)^{2}
\end{aligned}
$$

Using. equation (29) for the time dependence of the voltage, where now the total series resistance is equal to $\left(R_{d}+R_{L}\right)$, then:

$$
\begin{gathered}
\mathbf{P}_{\mathrm{RI}}(\mathbf{t})=\left[\mathbf{V}_{\mathrm{o}}{ }^{2} \mathbf{R}_{\mathrm{L}} /\left(\mathbf{R}_{\mathrm{d}}+\mathbf{R}_{\mathrm{L}}\right)^{2}\right] \exp \left[-2 \mathrm{t} /\left(\left(\mathbf{R}_{\mathrm{d}}+\mathbf{R}_{\mathrm{L}}\right) \mathbf{C}_{\mathrm{d}}\right)\right] \\
\mathbf{P}_{\mathbf{R L}}(\mathbf{t})=\left\{2 \mathbf{E}_{\mathrm{T}} \mathbf{R}_{\mathrm{L}} /\left[\left(\mathbf{R}_{\mathrm{d}}+\mathbf{R}_{\mathrm{L}}\right)^{2} \mathbf{C}_{\mathrm{d}}\right]\right\} \exp \left[-2 \mathrm{t} /\left(\left(\mathbf{R}_{\mathrm{d}}+\mathbf{R}_{\mathrm{L}}\right) \mathbf{C}_{\mathrm{d}}\right)\right] \\
\mathbf{P}_{\mathbf{R d}}(\mathbf{t})=\left[\mathbf{V}_{\mathrm{o}}{ }^{2} \mathbf{R}_{\mathrm{d}} /\left(\mathbf{R}_{\mathrm{d}}+\mathbf{R}_{\mathrm{L}}\right)^{2}\right] \exp \left[-2 \mathrm{t} /\left(\left(\mathbf{R}_{\mathrm{d}}+\mathbf{R}_{\mathrm{L}}\right) \mathbf{C}_{\mathrm{d}}\right)\right] \\
\mathbf{P}_{\mathbf{R d}}(\mathbf{t})=\left\{2 \mathbf{E}_{\mathrm{T}} \mathbf{R}_{\mathrm{d}} /\left[\left(\mathbf{R}_{\mathrm{d}}+\mathbf{R}_{\mathrm{L}}\right)^{2} \mathbf{C}_{\mathrm{d}} \mathrm{d}\right\} \exp \left[-2 \mathrm{t} /\left(\left(\mathbf{R}_{\mathrm{d}}+\mathbf{R}_{\mathrm{L}}\right) \mathbf{C}_{\mathrm{d}}\right)\right]\right.
\end{gathered}
$$

and the total power produced by the capacitor as a function of time is:

$$
\begin{gathered}
\mathbf{P}_{\text {cap }}(t)=\left[V_{o}^{2} /\left(R_{d}+R_{L}\right)\right] \exp \left[-2 t /\left(\left(R_{d}+R_{L}\right) C_{d}\right)\right] \\
P_{\text {cap }}(t)=\left\{2 E_{T} /\left[\left(R_{d}+R_{L}\right) C_{d}\right]\right\} \exp \left[-2 t /\left(\left(R_{d}+R_{L}\right) C_{d}\right)\right]
\end{gathered}
$$

The energy stored in the capacitor as a function time is, from equations (49) and (50), given by:

$$
\begin{gathered}
\mathbf{E}_{\text {cap }}(t)=(1 / 2) C_{d} V_{o}^{2} \exp \left[-2 t /\left(\left(R_{d}+R_{L}\right) C_{d}\right)\right] \\
E_{c a p}(t)=E_{T} \exp \left[-2 t /\left(\left(R_{d}+R_{L}\right) C_{d}\right)\right]
\end{gathered}
$$


If an additional resistor, $R_{L}{ }^{\prime}$, is connected in parallel with resistor $R_{L}$, then the influence of this additional resistor can be determined by substituting $R_{L} \equiv\left[R_{L} R_{L}^{\prime} /\left(R_{L}+R_{L}{ }^{\prime}\right)^{2}\right]$ in equations (53) through (62).

If the relationships given in equations (22) and (23) for the maximum power (at $1 / 3$ efficiency, i.e., $\sim 33.3 \%$ ) are used to redefine the above expressions for the power provided to a series load, $R_{L}$, i.e., $P_{d, \max }=V_{0}{ }^{2} /\left(4 R_{d}\right)$, and with the definitions of a reduced resistance, $r=R_{L} / R_{d}$, and a reduced time, $\tau=t /\left(R_{d} C_{d}\right)$ then the power delivered to the load in these reduced units is:

$$
\mathbf{P}_{\mathrm{RL}}(\tau)=\left[4 \mathbf{P}_{\mathrm{d}, \max }\right]\left[\mathbf{r} /(1+\mathbf{r})^{2}\right] \exp [-2 \tau /(1+\mathbf{r})]
$$

[Note that Miller [10] derived this expression in the referenced paper but it is in error as given.] Expression (63) can be used to examine the power delivered to a resistive load as a function of the maximum power deliverable from the capacitor (modeled with the series resistor, $R_{d}$ ). It should be emphasized that equation (63) with $P_{d, \max }$ defined as $V_{0}^{2} /\left(4 R_{d}\right)$ is for the case when the efficiency is $1 / 3$ or $-33.3 \%$. 
B-14 


\section{CAPACITOR TEST PROCEDURES (DC TESTS)}

\section{Constant-Current Test}

1. Determination of capacitor capacitance

2. Determination of equivalent series resistance (ESR).

3. The working energy (also known as the ideal stored energy) and power densities can also be determined.

4. Test cycle:

- Charge the capacitor at a constant specified current to the specified working voltage.

- Hold at this voltage for 10 to 20 seconds. (Note: It has been experimentally observed that for some ultracapacitor designs the hold time at constant voltage can have a deleterious effect on the subsequent performance of the capacitor. The mechanism(s) responsible for this effect have not been studied in detail.)

- Discharge the capacitor at a constant-current to a specified voltage (generally to 0 V).

- Hold at this voltage for 10 to 20 seconds.

These steps constitute one test cycle that is generally repeated three times. The maximum currents $\left(\mathrm{I}_{\text {test }}\right.$ in amperes) to use for these tests are generally kept such that the charge and discharge times are approximately equal to or greater than 10 seconds or by using a maximum discharge current as specified by the capacitor manufacturer.

If the voltage as a function of charge and discharge time follows the ideal-capacitor model [equations (3) and (4)], the capacitance of the capacitor can be calculated from the slope of the voltage versus charge or discharge time using:

$$
\mathbf{C}_{\text {cord }}=\left(\mathbf{I}_{\text {test, or d d }}\right)\left(\mathbf{t}_{\text {test, cor d }}\right) / \Delta \mathbf{V}_{\text {cor d }}
$$

where $\Delta V$ is the voltage range used in the test, $I_{\text {test }}$ is the constant-current used in the test, and thest is the measured time in seconds that it takes the capacitor to charge or discharge from the starting voltage to the ending voltage (usually $0 \mathrm{~V}$ to $\mathrm{V}_{\mathrm{o}}$ for a charging test and $\mathrm{V}_{\mathrm{o}}$ to $0 \mathrm{~V}$ for a discharging test). Equation (64) also assumes that the capacitance is a constant and is not a function of the voltage (or charge) on the capacitor, an assumption that may not be valid. The subscript "c or d" has been added to differentiate between a constant-current charge, "c", and a constant-current discharge, " $\mathrm{d}$ ". If the voltage behavior of the capacitor deviates significantly from that of an ideal-capacitor then the capacitance calculated using equation (64) can be seriously in error, especially if the charging voltage range does not go from zero volts to the working voltage, and the discharging voltage range does not go from the working voltage to zero volts. Actually, a better way to determine the charge and.discharge capacitance is to measure the total amount of charge (ampere-seconds or 3600 ampere-hours) charged into the capacitor during 
a constant-current charge, or discharged from it during a constant-current discharge (these values are generally not the same) and divide this value by the voltage change on the capacitor during the test, i.e.:

$$
C_{\text {cord }}=\Sigma[I(t) \Delta t]_{\text {ord }} d \Delta V_{\text {cord }}
$$

where $\Delta t$ is the time increment between the measured data points. As discussed in the previous section, the voltage dependence of the capacitance can be determined using the following equations:

$$
C_{c}\left(V_{c} \text { or } t\right)=\left[Q_{0}+Q\left(V_{c} \text { or } t\right)\right] /\left[V_{c}-I_{c} R_{c}\right]
$$

for charging and for discharging:

$$
C_{d}\left(V_{d} \text { or } t\right)=\left[Q_{0}-Q\left(V_{d} \text { or } t\right)\right] /\left[V_{d}+I_{d} R_{d}\right]
$$

where the charging capacitance, $C_{c}$, and discharging capacitance, $C_{d}$, as a function of the measured charge voltage, $V_{c}$, or the measured discharge voltage, $V_{d}$ (or the corresponding test time, $t$ ) can be determined if the corresponding initial charge on the capacitor before the start of the test, $Q_{0}$, and the constant-current charge current, $I_{c}$, and constant-current discharge current, $I_{d}$, as well as the experimentally determined equivalent series resistance for charging, $R_{c}$, and discharging, $R_{d}$, are known. Note that $R_{c}$ and $R_{d}$ usually depend on the constant-current charge and discharge current.

The working (also referred to as the ideal stored) energy density can be calculated using the definition for the energy in a capacitor that is $E=(1 / 2) Q V$, i.e., the energy is the product of the total charge stored in the capacitor times the voltage on the capacitor. This results in the expressions (assuming ideal capacitor behavior):

$$
\mathbf{E}_{\mathrm{w}} / \mathrm{Mass}=(1 / 2)\left(\mathrm{I}_{\text {test }} \mathbf{t}_{\text {test }} \Delta V\right) /(3600 \mathrm{M})
$$

in $\mathrm{W} \cdot \mathrm{h} / \mathrm{kg}$, where $\mathrm{M}$ is the mass of the capacitor in $\mathrm{kg}$ and the factor 3600 converts time in seconds to time in hours. The volumetric energy density is given by:

$$
\mathbf{E}_{\mathrm{w}} / \text { Volume }=(1 / 2)\left(\mathrm{I}_{\text {test }} \mathrm{t}_{\text {test }} \Delta \mathrm{V}\right) /\left(3600 \mathrm{~V}_{\text {cap }}\right)
$$

in $W \cdot h / L$, where $V_{\text {cap }}$ is the volume in liters of the capacitor. The values of $E_{w} / M a s s$ and $\mathrm{E}_{\mathrm{w}}$ / Volume may not be the same for the constant-current charge and discharge tests due to the fact that the capacitance and the ESR values may not be the same. A better method to use is to follow the approach used in equations (66) and (67) and sum the QV product from the experimental data such that:

$$
\begin{aligned}
\mathbf{E}_{\mathrm{w}} / \text { Mass } & =(1 / 2) \Sigma[\mathbf{I}(\mathbf{t}) \mathrm{V}(\mathbf{t}) \Delta t] /(3600 \mathrm{M}) \\
\mathbf{E}_{\mathrm{w}} / \text { Volume } & =(1 / 2) \Sigma[\mathbf{I}(\mathbf{t}) \mathrm{V}(\mathbf{t}) \Delta t] /\left(3600 \mathrm{~V}_{\text {cap }}\right)
\end{aligned}
$$


where $I(t)$ and $V(t)$ are, respectively, the time dependent current and the time dependent voltage during the test, and $\Delta t$ is the time increment between data points. Even though these are constant-current tests, the value of the current can vary somewhat during the test, depending on the stability of the tester, so I(t) is formally expressed in equations (70) and (71).

Considerations of the efficiency of the capacitor during a charge/discharge cycle can also be evaluated from the experimental data by defining the efficiency during constant-current operation of the capacitor to be:

$$
\epsilon_{\text {constant-current }}=\left\{\Sigma[I(t) V(t) \Delta t]_{\text {ced }}\right\} /\left\{\Sigma[I(t) V(t) \Delta t]_{\text {cec }}\right\}
$$

Equation (72) results from defining the efficiency to be the energy extracted from the capacitor during a constant-current discharge, $(1 / 2) \Sigma[\mathrm{I}(\mathrm{t}) \mathrm{V}(\mathrm{t}) \Delta \mathrm{t}]_{\mathrm{ccd}}$, divided by the energy stored in the capacitor during the constant-current charge, $(1 / 2) \Sigma[\mathrm{I}(\mathrm{t}) \mathrm{V}(\mathrm{t}) \Delta \mathrm{t}]_{\mathrm{ccc}}$. The voltage range over which the two energies are determined, and the magnitude of the charge/discharge current, should be the same for the charge and discharge test cycles in order that the evaluation of the efficiency is meaningful.

In the constant-current tests, the current, $\mathrm{I}$, is suddenly changed at the beginning of the charge/discharge portions of the test cycle. The equivalent series resistance (ESR) is calculated at the beginning of the charge and the discharge cycle using:

$$
\mathbf{E S R}=(\Delta \mathbf{V})_{\text {IR step }} / \Delta \mathbf{I}_{\text {IR step }}
$$

where $(\Delta \mathrm{V})_{\mathbb{R} \text { step }}$ is the voltage change that occurs during the change in current, $\Delta \mathrm{I}_{\mathrm{IR} \text { step }}$, at the beginning of the constant-current charging and discharging of the capacitor. The ESR value is given in ohms and is generally found to vary with the constant-current charge or discharge test value.

\section{Constant-Power Test}

1. Determination of the specific energy densities (W.h/kg and $\mathrm{W} \cdot \mathrm{h} / \mathrm{L}$ ) and specific power densities (W/kg and $\mathrm{W} / \mathrm{L})$.

2. Test cycle:

- The capacitor is charged at a specified constant-current to a specified final voltage.

- The capacitor is held at this voltage for 10 to 20 seconds. (As noted previously, the duration of the constant voltage hold can have an effect on the performance of the capacitor.)

- A constant-power discharge is started and the capacitor is discharged to a specified voltage [generally to $1 / 2$ the working voltage that results in the extraction of $3 / 4$ of the total energy available from the capacitor, refer to equations (11) and (12)]. 
- The capacitor is then held at this voltage for 10 to 20 seconds.

These steps constitute one test-cycle that is generally repeated three times.

For each constant-power discharge portion of the test cycle the energy ( 1 watt-second $=$ $3600 \mathrm{~W} \cdot \mathrm{h}=1$ joule) is calculated by summing the product VI $\Delta \mathrm{t}$ ( $\mathrm{V}$ in volts, $\mathrm{I}$ in amperes and $\Delta \mathrm{t}$ the duration of the time between measurement of $\mathrm{V}$ and $\mathrm{I}$, in seconds) during the discharge. The specific energy mass density (generally referred to as the specific energy) is then calculated using:

$$
\mathrm{E}_{s} / \text { Mass }=\Sigma[\mathrm{V}(\mathrm{t}) \mathrm{I}(\mathrm{t}) \Delta \mathrm{t}]_{\text {discharge }} /(3600 \mathrm{M})
$$

in $\mathrm{W} \cdot \mathrm{h} / \mathrm{kg}$, where $\mathrm{M}$ is the mass of the capacitor in $\mathrm{kg}$, and the specific energy volumetric density:

$$
E_{s} / \text { Volume }=\Sigma[V(t) I(t) \Delta t]_{\text {discharge }} /\left(3600 \mathrm{~V}_{\text {cap }}\right)
$$

in $\mathrm{W} \cdot \mathrm{h} / \mathrm{L}$ where $\mathrm{V}_{\text {cap }}$ is the volume of the capacitor in liters.

Under conditions of a constant-power test the specific power, $P_{s}$, is set by the test, i.e., the tester maintains the current $\cdot$ voltage, IV, product constant, and the specific power densities corresponding to the specific energy densities of equations (74) and (75) would be this power divided by the mass of the capacitor (in $\mathrm{kg}$ ) or the volume of the capacitor (in liters) respectively.

Considerations of efficiency can also be made for the results of constant-power tests [3]. In this case the round-trip efficiency, $\eta_{\mathrm{r}}$, has been defined to be [3]:

$$
\begin{gathered}
\eta_{\text {constant-power }}= \\
\left\{\Sigma[V(t) I(t) \Delta t]_{\text {constant-power discharge }\}} /\left\{\Sigma[V(t) I(t) \Delta t]_{\text {constant-current charge }}\right.\right.
\end{gathered}
$$

In equation (76) the variables are as previously defined. The energy term in the numerator is the energy extracted during the constant-power discharge. The energy term in the denominator is the energy stored in the capacitor during its constant-current charging. The upper and lower voltage levels of the two portions of the test cycle, i.e., the constant-current charge and the constantpower discharge, should be the same, or at least clearly specified when presenting the test results.

\section{Leakage-Current Test}

1. The leakage-current, $I_{L}(t)$, is the current required to maintain the capacitor at a specified voltage, $V_{o}$. The leakage-current is time dependent and only approaches a constant value after the voltage has been applied to the capacitor for, generally, a number of hours.

2. Determines the value of the parallel resistor, $R_{p}$, in the simple circuit model of the capacitor. 


$$
\mathbf{R}_{\mathrm{p}}(\mathbf{t})=\mathbf{V}_{\mathrm{o}} \mathbf{I}(\mathbf{t})
$$

where $V_{o}$ is the initial voltage on the capacitor and $I(t)$ is the measured time-dependent current required to maintain the voltage at its initial value. The value reported for $R_{p}$ is when $V_{0} / I(t)$ has reached a relatively constant value.

3. Test cycle:

- The capacitor is charged to a specified voltage using a specified constant-current and maintained at that voltage for approximately twenty-four hours or until the current required to maintain the voltage has come to a stable value.

- The current required to maintain the capacitor at the specified voltage should be measured with a tester that has the capability to measure small currents (fractions of a milliampere). Generally, measurements are made at one-minute intervals for the first hour and then at five-minute intervals during the remainder of the test.

\section{Self-Discharge Test (also known as the Stand-Test)}

1. Measures the time dependence of the self-dissipation of the capacitor, i.e., the rate of those internal processes that cause the capacitor to discharge when not connected to a load.

2. Measures the time dependent self-discharge energy loss factor $\operatorname{SDLF}(t)$ that results from measuring the time dependence of the capacitor voltage, $\mathrm{V}(\mathrm{t})$, when the capacitor is not connected to an external load:

$$
\operatorname{SDLF}(t)=1-\left[V(t) / V_{o}\right]^{2}
$$

where $\mathrm{V}_{\mathrm{o}}$ is the initial voltage on the capacitor. The energy removed from the capacitor is given by [from equation (11)]:

$$
\begin{gathered}
\mathbf{E}_{\text {removed }}(t)=(1 / 2) C V_{0}^{2}\left\{1-\left[V(t) / V_{0}\right]^{2}\right\} \\
E_{\text {removed }}(t)=(1 / 2) C V_{0}^{2}[\operatorname{SDLF}(t)] \\
\mathbf{E}_{\text {removed }}(t)=\mathbf{E}_{\mathrm{T}}[\operatorname{SDLF}(t)]
\end{gathered}
$$

The SDLF(t) value permits the determination of the amount of energy remaining in the capacitor [by using equations (11) and (13)] after it has been charged to a voltage $V_{o}$ and then placed in a condition where it is not connected to an external load. The total energy originally stored in the capacitor is from equation (13), $\mathrm{E}_{\mathrm{T}}=(1 / 2) \mathrm{CV}_{0}^{2}$, so that the energy remaining in the capacitor as a function of time is:

$$
\begin{gathered}
E_{\text {remaining }}(t)=E_{T}-E_{\text {removed }}(t) \\
E_{\text {remaining }}(t)=E_{T}[1-\operatorname{SDLF}(t)]
\end{gathered}
$$




$$
\mathbf{E}_{\text {remaining }}(t)=(1 / 2) C V(t)^{2}
$$

with $V(t)$ being the test time dependent voltage measured during the self-discharge test. The capacitance, $\mathrm{C}$, to be used in this case is somewhat problematic as it is known from experimental studies that the capacitance can be a function of the discharge current and, perhaps, even highly dependent on the voltage (charge) of the capacitor. As a first approximation for $\mathrm{C}$, a reasonable approach would be to extrapolate the current-dependent capacitance to its value at a discharge current of zero and use this value for $\mathrm{C}$ in equation (84).

\section{Test cycle:}

- The capacitor is charged to a specified voltage, $\mathrm{V}_{0}$, using a specified constantcurrent charge and held at that voltage for 30 minutes. (Note: the influence of the hold time on the subsequent performance of the capacitor has not been studied in detail.)

- The capacitor is then placed on open-circuit and the voltage measured over a period of approximately 72 hours, or as specified. The measuring equipment should have a high input impedance in order to minimize its effects on the capacitor discharge.

- $\quad$ For the first three hours, the time increment between voltage measurements should be one minute or less. For the remainder of the test, measurements should be made every 10 minutes or less.

\section{Life-Cycle Tests}

1. This test measures capacitor performance stability during cyclic constant-current charging and constant current discharging tests.

2. Test Cycle:

- Condition the capacitor at $25 \pm 3^{\circ} \mathrm{C}$ until thermal equilibrium is reached. Initialize the voltage on the capacitor at a specified voltage that is generally $1 / 2$ of the specified working voltage of the capacitor.

- Then charge the capacitor at a constant-current that corresponds to that required to raise the voltage on the capacitor to its specified working voltage in $18 \pm 1$ seconds. The appropriate constant-current charging current can be determined from the Constant-Current Test.

- Maintain this voltage on the capacitor for $10 \pm 0.5 \mathrm{~s}$.

- Then discharge the capacitor to one-half the working voltage using a constantcurrent discharge with the same current used during the charging cycle and hold at this voltage for $10 \pm 0.5 \mathrm{~s}$. 
- This defines one test cycle. Repeat this cycle throughout the testing, adjusting the charging and discharging currents as needed in order to maintain the initial charge/discharge times of $18 \pm 1 \mathrm{~s}$. This results in a cycle time of approximately 56 seconds.

- Take data for the first ten cycles and then every 500 cycles thereafter.

- Check data files each day to determine cycle time. Adjust current to maintain the 56 second cycle to within $5 \%$. The charge and discharge current will generally have to be decreased as the capacitance value for the capacitor generally decreases during life-cycle testing.

- Terminate the test when the charging and discharging watt-hour values have decreased to $80 \%$ of their initial values. This implies that the test should be stopped when the constant-current charge and discharge currents are $80 \%$ of their initial values. 


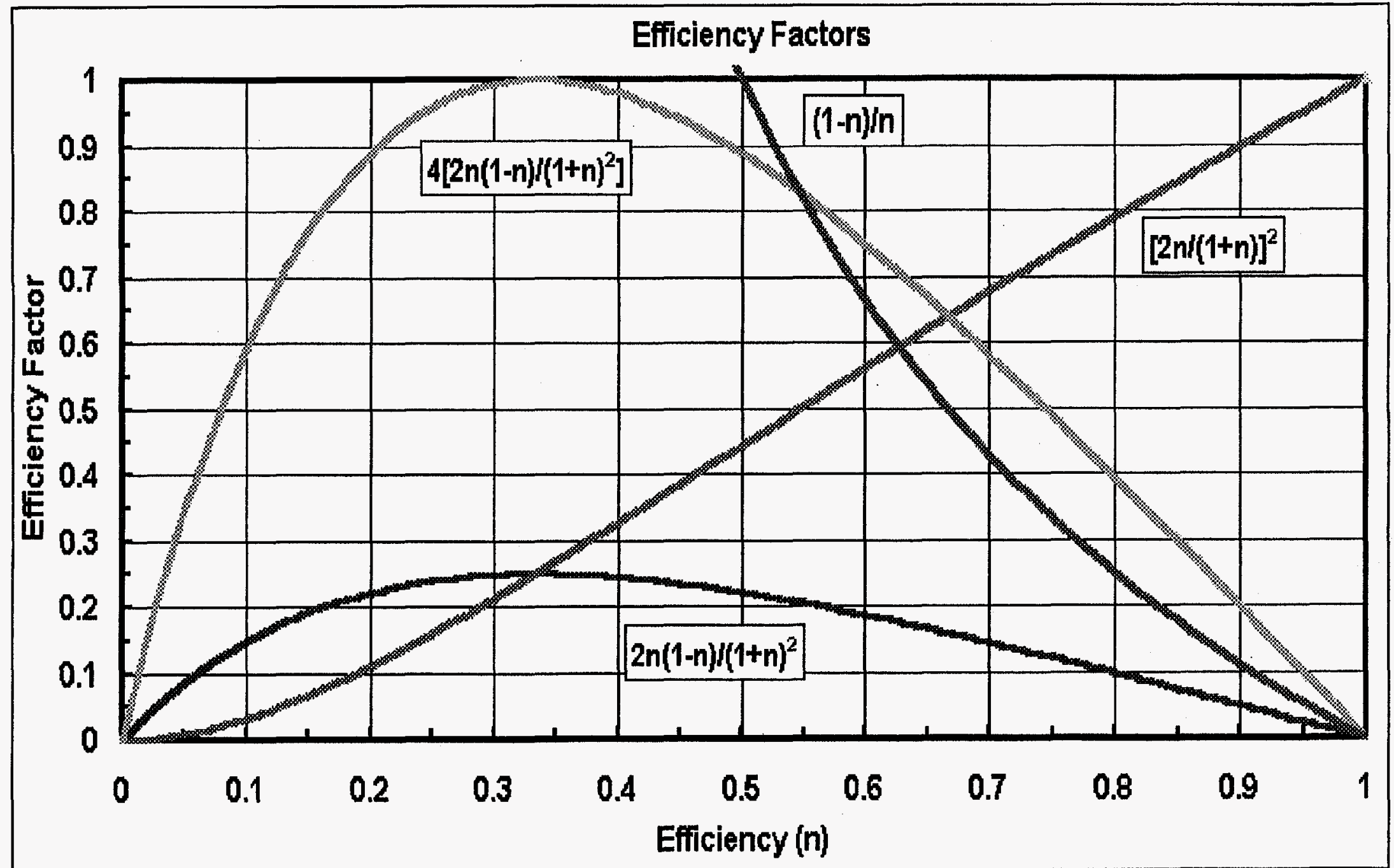

Figure B-1. Plots of the various efficiency factors as discussed in Appendix B as a function of the efficiency. 


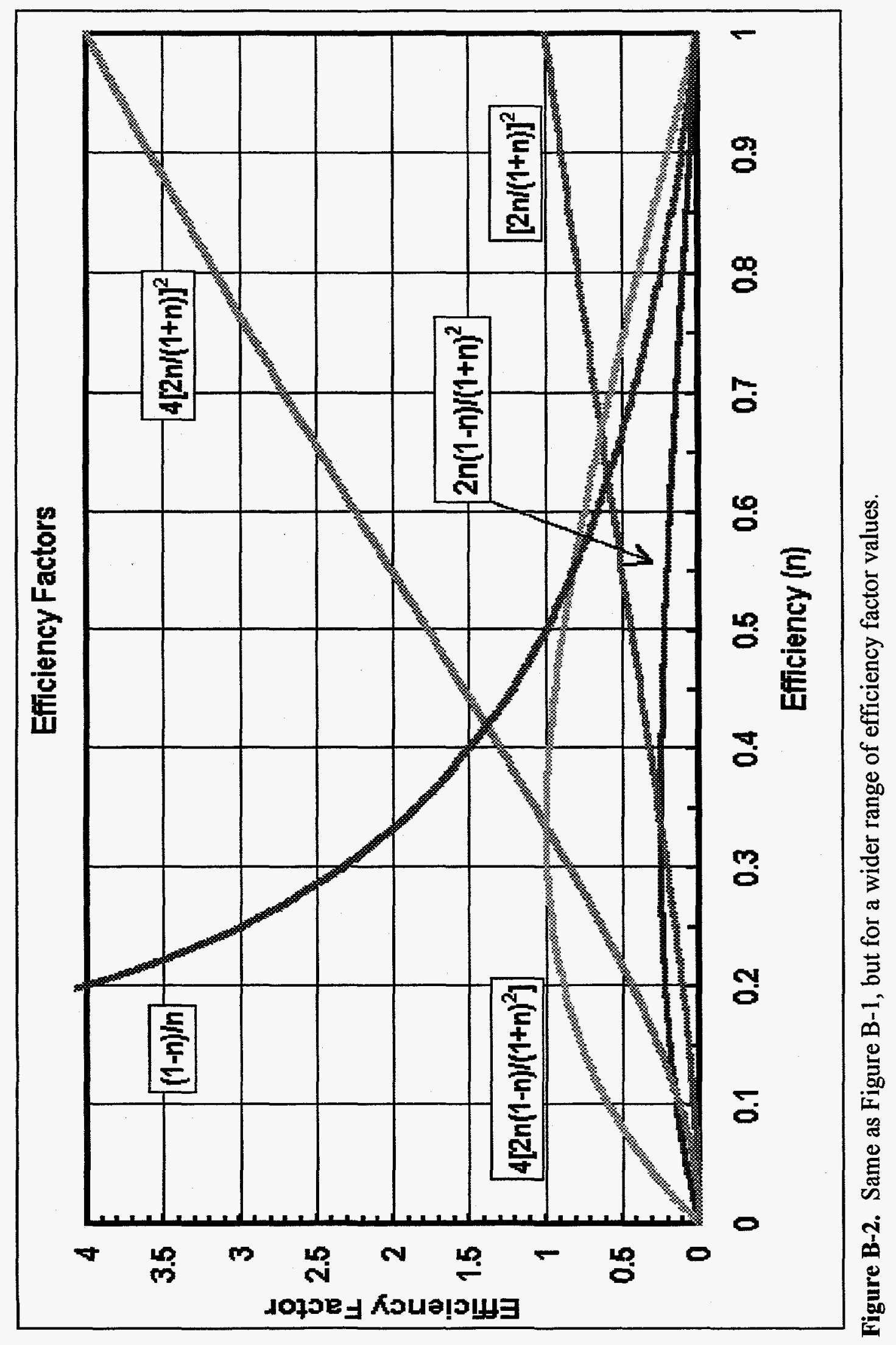




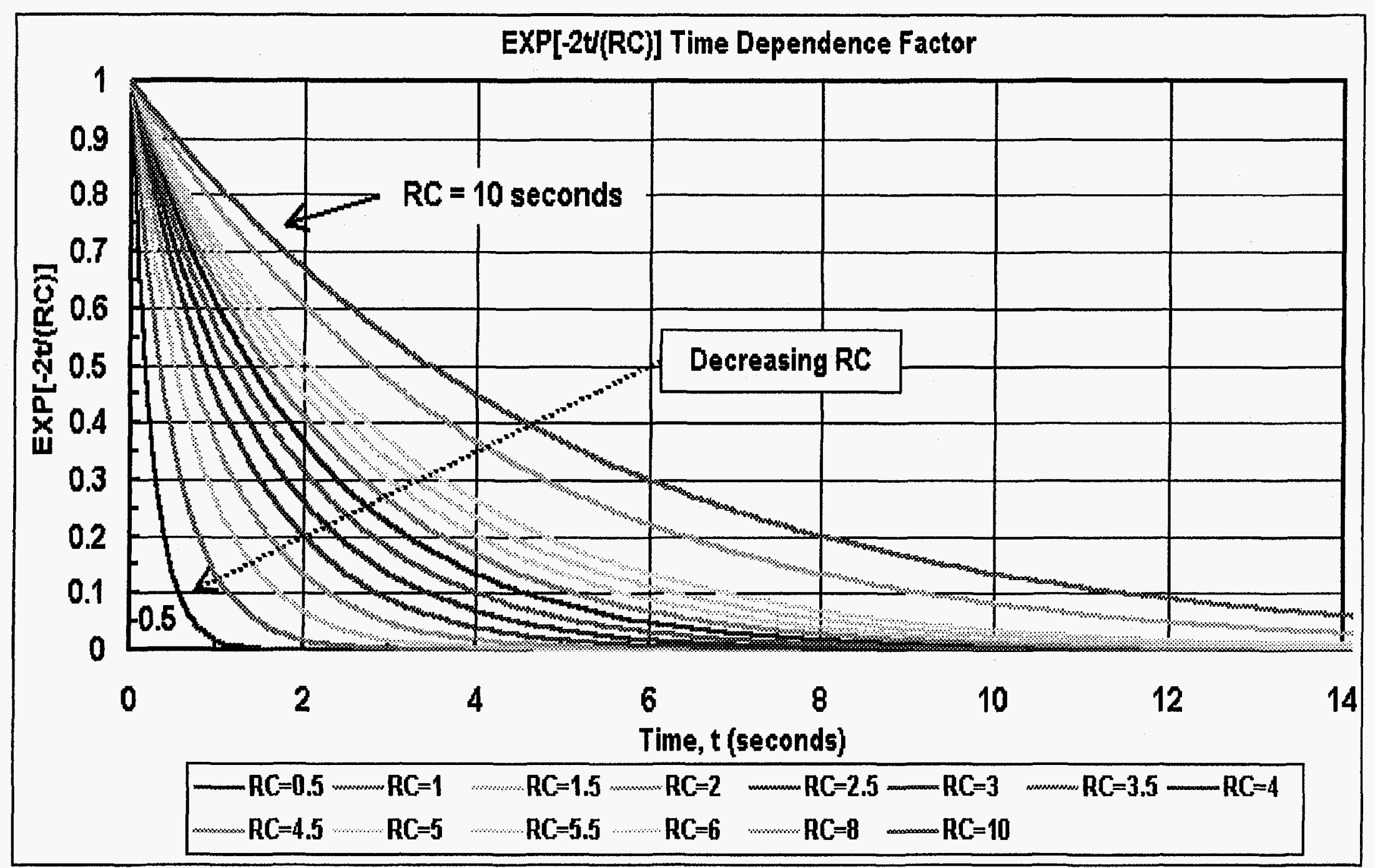

Figure B-3. Plots of the exponential time dependence factor $(\operatorname{EXP}[-2 t /\{R C\}])$ as a function of time $(t)$ for various values of $R C$. 